\title{
Raster-Thermospannungs-Mikroskopie der Interferenz von Elektronenwellen auf der Au(111)-Oberfläche
}

\author{
Dissertation \\ zur Erlangung des Doktorgrades \\ der Mathematisch-Naturwissenschaftlichen Fakultäten \\ der Georg-August-Universität zu Göttingen
}

vorgelegt von

Klaus Jürgen Engel

aus Rheydt

Göttingen 2001 
D 7

Referent: Prof. Dr. R. G. Ulbrich

Korreferent: Prof. Dr. K. Schönhammer

Tag der mündlichen Prüfung: 19. Oktober 2001 


\section{Inhaltsverzeichnis}

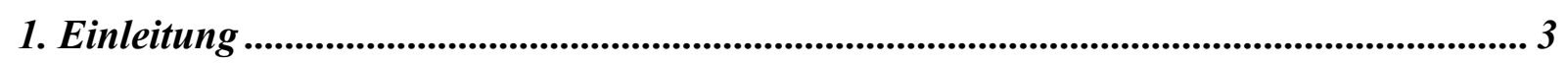

2. Rastertunnelmikroskopie ...............................................................................................6 6

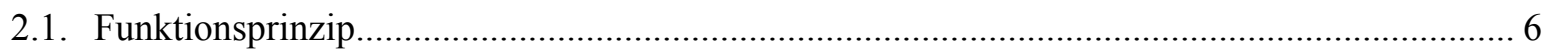

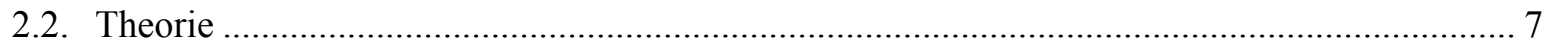

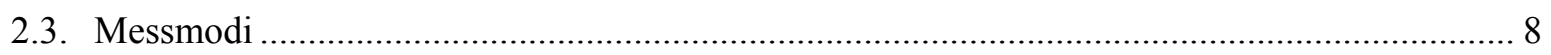

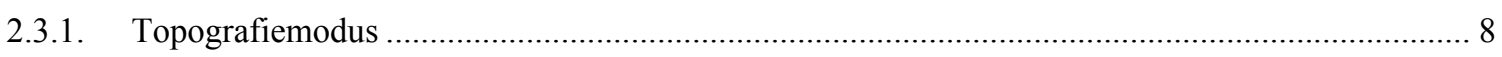

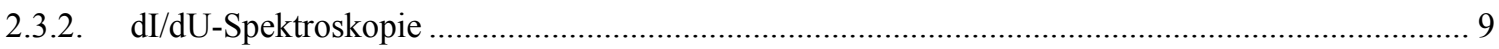

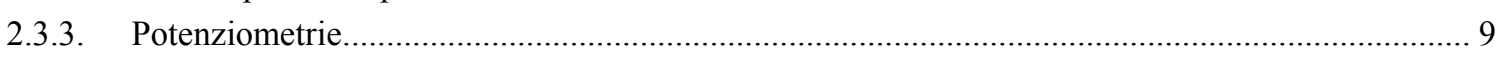

3. Raster-Thermospannungs-Mikroskopie......................................................................... 10

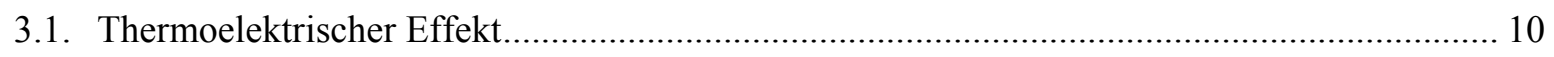

3.2. Thermoelektrischer Effekt am Tunnelkontakt ................................................................. 11

3.3. Erstellung von Thermospannungs-Karten mit dem STM ................................................ 14

4. Experimenteller Aufbau................................................................................................... 16

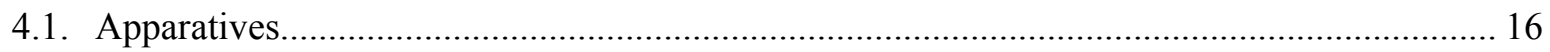

4.1.1. $\quad$ UHV-Tieftemperatur-STM ..................................................................................... 16

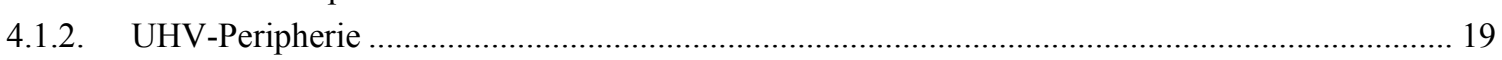

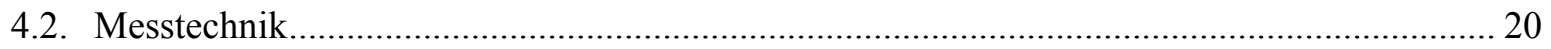

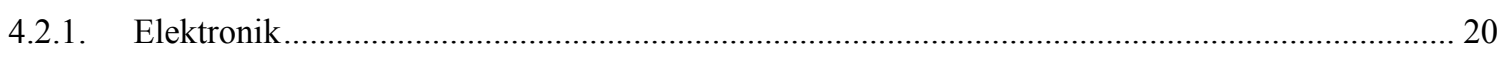

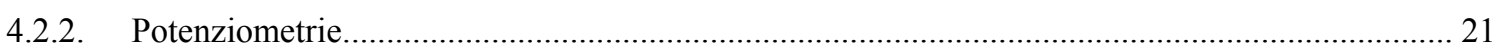

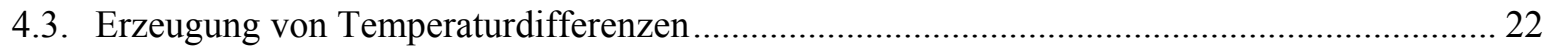

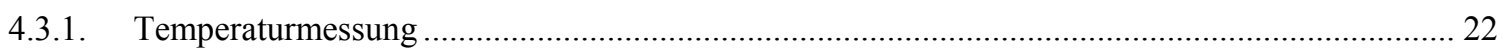

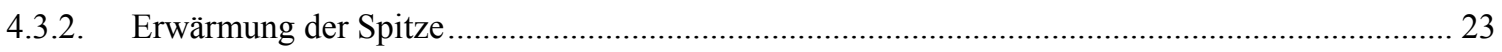

4.3.3. $\quad$ Wärmeleitung der Tunnelbarriere ...................................................................................... 23

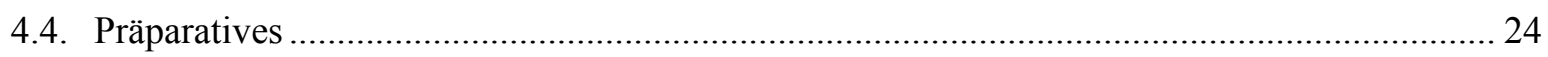

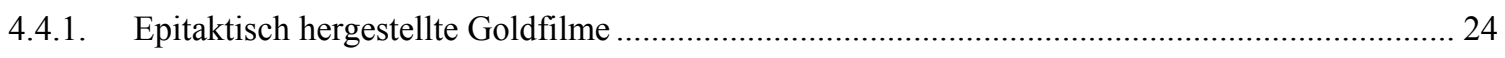

4.4.2. $\quad$ Präparation des $\mathrm{Au}(111)$-Einkristalls ....................................................................... 26

4.4.3. $\quad$ Spitzenpräparation...................................................................................................... 30

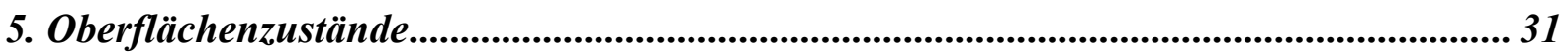

6. Die Au(111)-Oberfläche............................................................................................................... 34

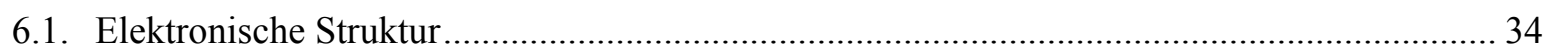

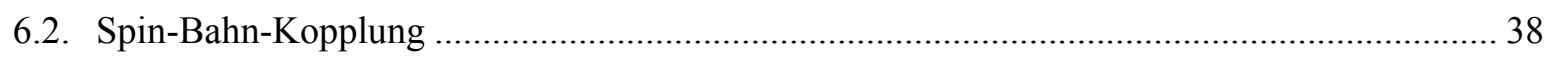

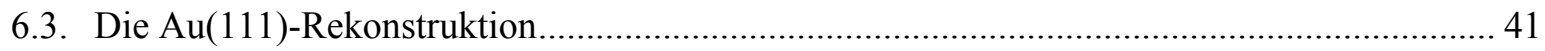

7. Interferenz von Elektronenwellen ............................................................................................. 45

7.1. Lokale Zustandsdichte an geradlinigen Stufen .......................................................... 45

7.2. Lokale Zustandsdichte für allgemeinere Geometrien.................................................... 47 
8. Thermospannungs-Oszillationen an geradlinigen Stufen............................................... 49

8.1. Experimentelle Ergebnisse ......................................................................................... 49

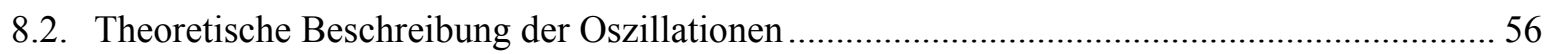

8.2.1. Grafische Darstellung der LDOS ........................................................................ 57

8.2.2. $\quad$ Analytische Behandlung im Tersoff-Hamann-Modell ......................................................... 58

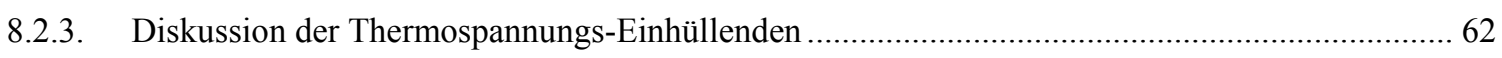

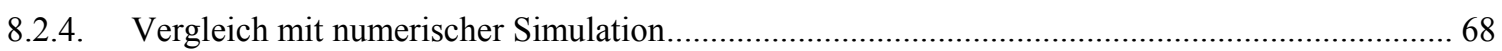

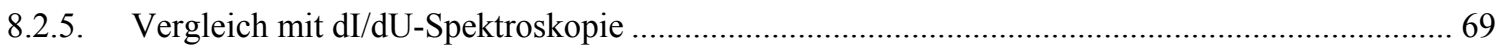

8.2.6. $\quad$ Einfluss der Spitzen- und der lokalen Volumen-Zustandsdichte ................................................ 69

8.3. Bestimmung von Kohärenzlängen aus dem Interferenzmuster ...................................... 72

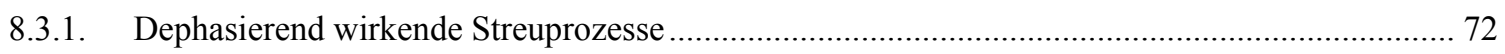

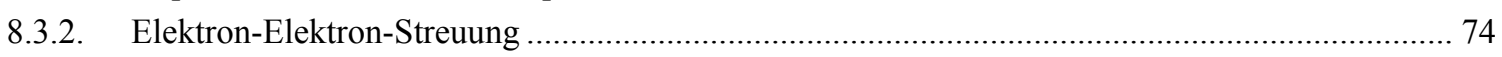

8.3.3. $\quad$ Elektron-Phonon-Streuung .............................................................................................. 76

8.3.4. Abklinglängen in Interferenzmustern ............................................................................. 77

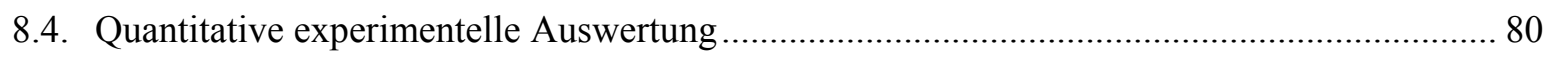

9. Thermoelektrische Effekte in der Topografie ..................................................................86

10. Monatomare Stufen als Beugungsgitter............................................................................ 91

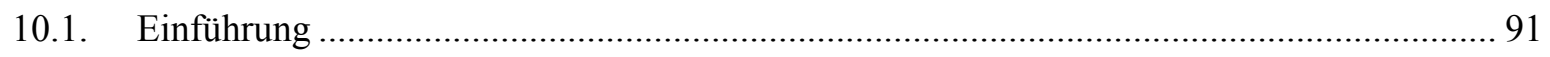

10.2. Die Rekonstruktion: Confinement für Oberflächenzustände? ......................................... 91

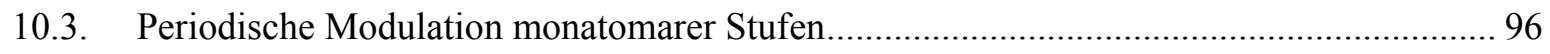

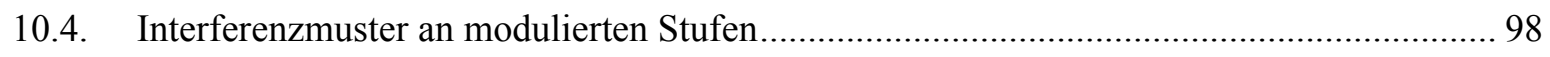

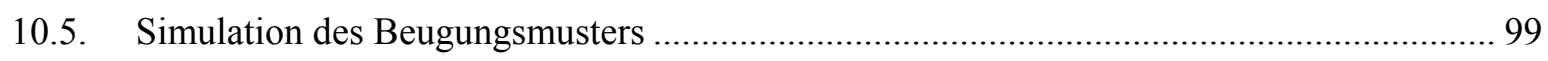

10.5.1. Technisches und grafische Darstellung ............................................................................ 100

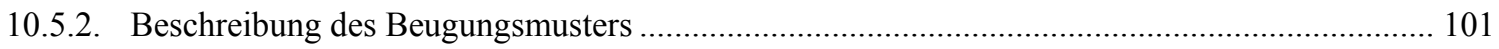

10.5.3. Variation der Stufenparameter ..................................................................................... 102

10.6. $\quad$ Vergleich und Neuinterpretation experimenteller Daten ............................................. 107

11. Zusammenfassung................................................................................................................. 109

Anhang A: Lokale Zustandsdichte an geradlinigen Stufen ............................................. 111

Anhang B: Analytische Herleitung der Oszillationseinhüllenden im Tersoff Hamann Modell......................................................................................................................................................... 114

Anhang C: Näherung der Besselfunktion in Fermi-Integralen ......................................... 123

Anhang D: Analytische Bestimmung der Topografie........................................................... 125

Verzeichnis verwendeter Formelzeichen und Abkürzungen ............................................... 128

Literaturverzeichnis............................................................................................................................ 131

Danksagung........................................................................................................................................... 139

Lebenslauf ................................................................................................................................... 141 


\section{Einleitung}

Die Interferenz von Wellen ist ein Phänomen, welches auf den verschiedensten Gebieten der Physik zu finden ist. Je nach Phasenlage überlagern sich Wellen, wenn sie aufeinandertreffen, entweder konstruktiv oder destruktiv. Es entstehen Interferenzmuster.

Ein Meilenstein in der Entwicklung der Quantenmechanik war die Erkenntnis, dass auch Materie Welleneigenschaften besitzt [deB] Ab diesem Zeitpunkt betrachtete man Elektronen nicht mehr als punktförmige Objekte, die allein der Newtonschen Mechanik gehorchten. Vielmehr wurde ihnen eine Wellenfunktion zugeordnet, deren Betragsquadrat ein Maß für die Aufenthaltswahrscheinlichkeit des Elektrons an einem bestimmten Ort angibt. Ähnlich wie Wasserwellen, welche bei der Reflexion an einer Mauer Schwingungsknoten und -Bäuche aufweisen, besitzt auch das Betragsquadrat von reflektierten Elektronenwellen ein Interferenzmuster.

Die Entwicklung der Rastertunnelmikroskopie von G. Binnig und H. Rohrer im Jahre 1982 [Bin 1-3] ermöglichte eine direkte Beobachtung solcher quantenmechanischer Effekte im Ortsraum mit atomarer Auflösung. Pionierarbeit leisteten hier D. Eigler und Mitarbeiter, als sie 1993 erstmals die Interferenz von Elektronenwellen auf einer Kupfer-Oberfläche beobachten [Cro 1], Etwa zeitgleich untersuchten Y. Hasegawa und P. Avouris ähnliche Interferenzerscheinungen auf Gold-Oberflächen [Has 1], Größte Aufmerksamkeit erlangte die Gruppe um D. Eigler, als es ihr gelang, eine quantenmechanische „Spielwiese“ durch gezielte Manipulation einzelner Atome und deren Anordnung zu sogenannten „Quantum corrals“ zu erschaffen [Cro 2-4, Hel 1-3, Man]. Es war gelungen, quantenmechanische Eigenzustände von Elektronen in aus Atomen geformten Resonatoren zu beobachten. In der Folgezeit gab es eine Vielzahl von Arbeiten, welche die Interferenz von Elektronenwellen mit dem Rastertunnelmikroskop auf diversen Materialien untersuchten. Hierbei konnten Erkenntnisse über Streuung und Abschirmung [SW 1] sowie die Entstehung von Eigenzuständen in räumlich begrenzten Potenzialen [SW 2] gewonnen werden. Aus dem Interferenzmuster erhielt man Informationen über die Bandstruktur [SW 3], der Topologie der Fermioberfläche [SW 4] und der Dynamik angeregter Zustände [Bür 3-4, Cro 2, Jea]. Es wurden auch Wechselwirkungseffekte zwischen Oberflächenzuständen und Adsorbaten gefunden [Höv, Par, Rep. Wah.

In dieser Arbeit wird die Interferenz von Elektronenwellen auf der (111)-Oberfläche von Gold untersucht. Die Kartierung der Interferenzmuster erfolgt mittels Raster-ThermospannungsMikroskopie, bei der ortsaufgelöst die am Tunnelkontakt abfallende Thermospannung aufgezeichnet wird.

Nach einer Einführung in die Rastertunnelmikroskopie in Kapitel 2 wird speziell auf den thermoelektrischen Effekt am Tunnelkontakt in Kapitel 3 eingegangen. Diese bisher noch 
wenig verbreitete Technik besitzt eine hohe Empfindlichkeit auf Variationen in der lokalen elektronischen Struktur der Oberfläche. Kapitel 4 stellt den experimentellen Aufbau und die Präparation vor.

Kapitel 5 gibt einen Überblick über Oberflächenzustände und deren Eigenschaften.

Kapitel 6 behandelt die Au(111)-Oberfläche, welche sich durch zwei Besonderheiten auszeichnet. Zum einen gibt es ein parabolisches Band von Oberflächenzuständen, welche ein nahezu ideales zweidimensionales Gas von freien Elektronen bilden. Die Elektronen können durch ebene zweidimensionale Wellen beschrieben werden, welche an Oberflächendefekten wie z.B. monatomaren Stufen gestreut werden.

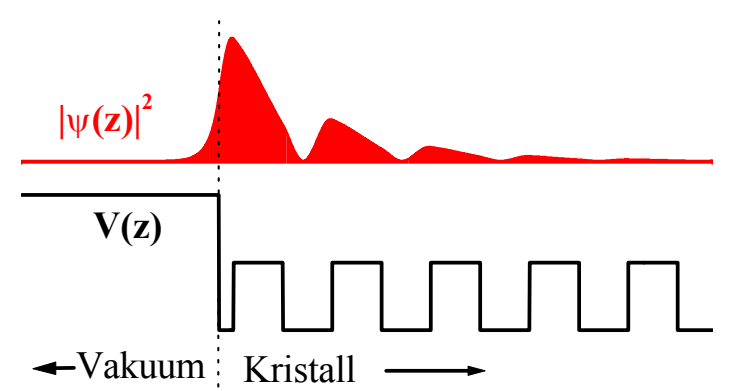

Abb. 1.1 Schematische Darstellung des Betragsquadrates des Oberflächenzustandes von $\mathrm{Au}(111)$. Oberflächenzustände besitzen Energien, welche innerhalb einer Volumen-Bandlücke liegen, so dass Elektronen in diesen Zuständen weder in den Kristall hinein propagieren noch denselben verlassen können.

Die zweite Besonderheit ist die sogenannte „Herringbone-Rekonstruktion“, welche ein Muster von quasi-eindimensionalen Domänen unterschiedlicher Stapelfolgen bildet.

Kapitel 7 stellt Methoden zur Berechnung von Interferenzmustern vor.

In Kapitel 8 werden experimentelle Messungen von Interferenzmustern an OberflächenStufen vorgestellt, welche unter Anwendung der Raster-Thermospannungs-Mikroskopie erstmals bei Temperaturen um $80 \mathrm{~K}$ untersucht und charakterisiert werden. Während das Interferenzmuster bei topografischen oder spektroskopischen Messungen immer stehende Wellen zeigt, welche mit dem Abstand von der Stufe monoton abklingen [SW 1], wird demgegenüber in der Raster-Thermospannungs-Mikroskopie eine Besonderheit beobachtet: Die Amplitude der stehenden Wellen nimmt mit dem Abstand von der Stufe zunächst zu, bis bei einem durch die Temperatur definierten charakteristischen Abstand ein maximaler Kontrast beobachtet wird. In dieser Arbeit ist es erstmals gelungen, das Interferenzmuster in der Thermospannung im Modell analytisch zu beschreiben und eine qualitative wie auch quantitative Charakterisierung der experimentellen Einhüllenden zu geben. Aus der Lage des Kontrastmaximums bzw. den Abklingkonstanten der experimentell gemessenen Thermospannungsamplituden ist es möglich, Kohärenzlängen und Lebenszeiten von Oberflächenzuständen bei der Fermienergie zu bestimmen.

Kapitel 9 untersucht den thermoelektrischen Effekt im „herkömmlichen“ Topografiemodus eines Rastertunnelmikroskops. Es kann experimentell gezeigt werden, dass das thermoelektrische Signal in der Topografie wiederzufinden ist und bei niedrigen Tunnelspannungen zu Korrugationen in der Topografie führt, welche deutlich höher liegen als bei einem System mit 
gleicher Spitzen- und Probentemperatur. Dieser Effekt kann zu einer Neuinterpretation vieler Messergebnisse führen, welche vor allem bei Rastertunnelmikroskopen mit variabler Temperatur entstanden sind.

In Kapitel 10 wird gezeigt, wie eine periodisch modulierte Oberflächenstufe als Beugungsgitter für Elektronenwellen wirkt. Die periodischen Modulationen der Stufe sind von der Rekonstruktion hervorgerufen. Die experimentell gemessenen Interferenzmuster zeigen Kontrastmodulationen, welche in Simulationsrechnungen verifiziert werden. Diese Ergebnisse lassen eine Neuinterpretation der Wechselwirkung zwischen Rekonstruktion und Oberflächenzustände zu. Bisher wurde aufgrund topografischer Daten von Interferenzmustern angenommen, dass die Rekonstruktionsdomänen als eindimensionales „ConfinementPotenzial“ für Oberflächenzustände wirken [Fuj 1-3] Dieses Modell kann in dieser Arbeit zumindest für Zustände um die Fermienergie experimentell nicht bestätigt werden. Die Annahme einer Stufe als Beugungsgitter kann hingegen auch ältere topografische Daten hinreichend erklären.

Im Kapitel 11 wird schließlich eine Zusammenfassung dieser Arbeit gegeben. 


\section{Rastertunnelmikroskopie}

\subsection{Funktionsprinzip}

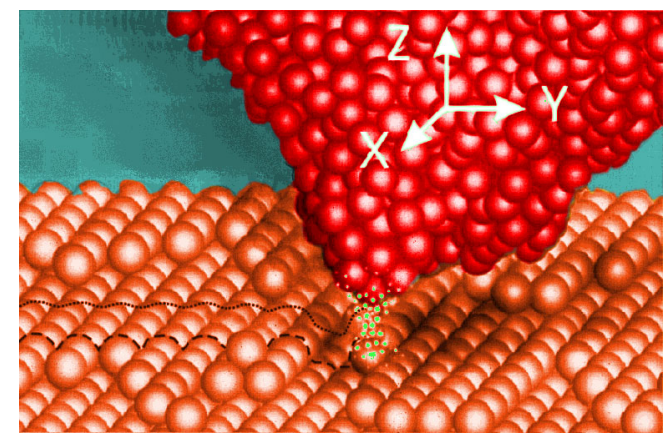

Abb. 2.1 Prinzip eines Rastertunnelmikroskops

(reproduziert aus Ref. G. Binnig, [Bin 3])

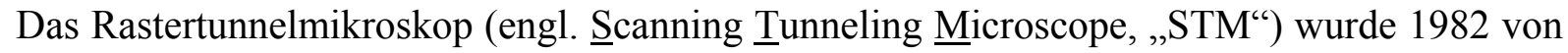
G. Binnig und H. Rohrer entwickelt [Bin 1-3] und bildete das Grundprinzip für eine Reihe von weiteren sogenannten Rastersondenmikroskopen [Bin 3, Wie].

Bei einem STM zeichnet eine metallische Spitze zeilenweise die Oberflächenkontur! (,Topografie“) einer Probe nach. Die Spitze kann dabei mit Hilfe von Piezo-Stellelementen in alle drei Raumrichtungen bewegt werden. Zur Abstandskontrolle wird der quantenmechanische Tunneleffekt benutzt [Gia, Fli]. Bei Annäherung der Spitze an die Oberfläche überlappen sich die Wellenfunktionen von Spitze und Probe. Dies ermöglicht das „Tunneln“ von Elektronen, selbst wenn die Spitze noch einige $\AA$ von der Oberfläche entfernt ist. Bei Anlegen einer elektrischen Spannung ist dieser Tunnelstrom exponentiell vom Abstand zwischen Probe und Spitze abhängig [Bar 1, Che 3]. Er erlaubt es, die Spitze mit einer Genauigkeit von wenigen $\mathrm{pm}$ zu positionieren.

Im Extremfall besteht die Spitze an ihrem äußersten Ende aus einem einzelnen Atom, über welches der überwiegende Teil des Tunnelstromes fließt. Das STM ermöglicht also, die Ladungsdichte einer Oberfläche lokal mit atomarer Auflösung zu untersuchen.

\footnotetext{
${ }^{1}$ Die „Oberflächenkontur“ wird erst durch das Messverfahren genau definiert. Näherungsweise handelt es sich um eine Fläche konstanter elektronischer Ladungsdichte.
} 


\subsection{Theorie}

Zur Interpretation von STM-Messungen ist eine theoretische Beschreibung des Tunnelstromes $\mathrm{I}_{\mathrm{T}}$ zwischen Spitze und Probe nötig. Hierfür wird die Verteilungsfunktion für Elektronen in Festkörpern benötigt, welche im thermodynamischen Gleichgewicht durch die Fermifunktion $\mathrm{f}(\varepsilon, \mathrm{T})$ beschrieben wird [Ash].

$$
f(\varepsilon, T)=\left[1+\exp \left(\frac{\varepsilon}{k_{B} T}\right)\right]^{-1} .
$$

Hier gibt $\varepsilon$ die Energiedifferenz zum elektrochemischen Potenzial $\mu_{\mu_{\mathrm{ec}}}$ und $\mathrm{T}$ die Temperatur an.

Die Spitze wie auch die Probe bei einem STM werden unabhängig voneinander als zwei Elektronenreservoire im thermodynamischen Gleichgewicht behandelt, zwischen denen Elektronenaustausch über die Tunnelbarriere möglich ist ${ }^{3}$ Wenn am Tunnelkontakt eine elektrische Spannung angelegt wird, dann verschieben sich die elektrochemischen Potenziale von Spitze und Probe um einen Betrag e.U gegeneinander. Wenn $\varepsilon$ die Energiedifferenz zum elektrochemischen Potenzial der Probe darstellt, dann wird die Fermifunktion der Probe durch $\mathrm{f}\left(\varepsilon, \mathrm{T}_{\mathrm{P}}\right)$ und die der Spitze durch $\mathrm{f}\left(\varepsilon+e \mathrm{e}, \mathrm{T}_{\mathrm{S}}\right)$ angegeben $\left(\mathrm{T}_{\mathrm{P}}\right.$ und $\mathrm{T}_{\mathrm{S}}$ sei jeweils die Temperatur der Probe bzw. der Spitze).

Eine weitere grundlegende Größe in der theoretischen Behandlung des Tunnelstromes ist die

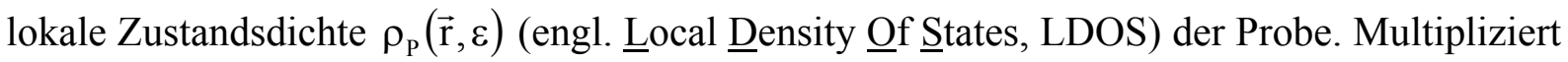
mit der Fermifunktion gibt die LDOS an, mit welcher Wahrscheinlichkeit man am Ort $\vec{r}$ ein Elektron mit der Energie $\varepsilon$ findet. Die LDOS $\rho_{\mathrm{P}}(\overrightarrow{\mathrm{r}}, \varepsilon)$ ist also die Summe der Betragsquadrate aller Wellenfunktionen $\psi_{v}(\vec{r})$ mit Energie $\varepsilon_{v}=\varepsilon$ am Ort $\overrightarrow{\mathrm{r}}$ :

$$
\rho_{\mathrm{P}}(\overrightarrow{\mathrm{r}}, \varepsilon)=\sum_{v}\left|\psi_{v}(\overrightarrow{\mathrm{r}})\right|^{2} \cdot \delta\left(\varepsilon_{v}-\varepsilon\right) .
$$

Die Summation läuft hier über alle Quantenzahlen v. Die $\delta$-Funktion stellt sicher, dass nur Zustände mit einer Eigenenergie $\varepsilon_{v}=\varepsilon$ in die LDOS eingehen.

\footnotetext{
${ }^{2}$ Das elektrochemische Potenzial $\mu_{\mathrm{ec}}$ ist definiert zu $\mu_{\mathrm{ec}}=\mu_{\mathrm{c}}-\mathrm{e} \cdot \mathrm{V}$, wobei $\mu_{\mathrm{c}}$ das chemische Potenzial und V das elektrische Potenzial für ein Elektron darstellt. Eine detaillierte Betrachtung dieser Thematik ist in [Eng 2, Sch 1] zu finden.

${ }^{3}$ Strenggenommen stört der Tunnelkontakt das thermodynamische Gleichgewicht sowohl in der Spitze wie auch in der Probe, da über die Tunnelbarriere Ladungsträger in das jeweils andere System injiziert werden. Diese Störung ist jedoch vernachlässigbar gering: Ein Würfel der Kantenlänge $0.2 \mathrm{~nm}$ wird in $\mathrm{Au}$ von ca. $5 \cdot 10^{17}$ Leitungselektronen pro Sekunde frequentiert [Iba]. Selbst wenn der gesamte Tunnelstrom von üblicherweise $1 \mathrm{nA}$ über diesen Würfel fließt, ergibt dies eine injizierte Störung von ca. $6 \cdot 10^{9}$ Elektronen pro Sekunde. Der relative Anteil injizierter „Stör“-Elektronen liegt also in einer Größenordnung von $10^{-8}$.
} 
Die Grundlagen zur Berechnung des Tunnelstrom zwischen zwei Elektroden gehen auf J. Bardeen zurück [Bar 1, Che 3]. Hierauf aufbauend entwickelten J. Tersoff und D. R. Hamann [Ter 1-3] eine theoretische Beschreibung für das STM. Für die Spitze werden die Wellenfunktionen als s-artige Kugelwellen angenommen, welche um die Position $\vec{r}$ des äußersten Atoms der Spitze zentriert sind. Senkrecht zur Probenoberfläche wird ein exponentielles Abklingen der LDOS angenommen. In diesem Fall kann gezeigt werden [Ter 1-3], dass der Tunnelstrom eine relativ einfache Form annimmt:

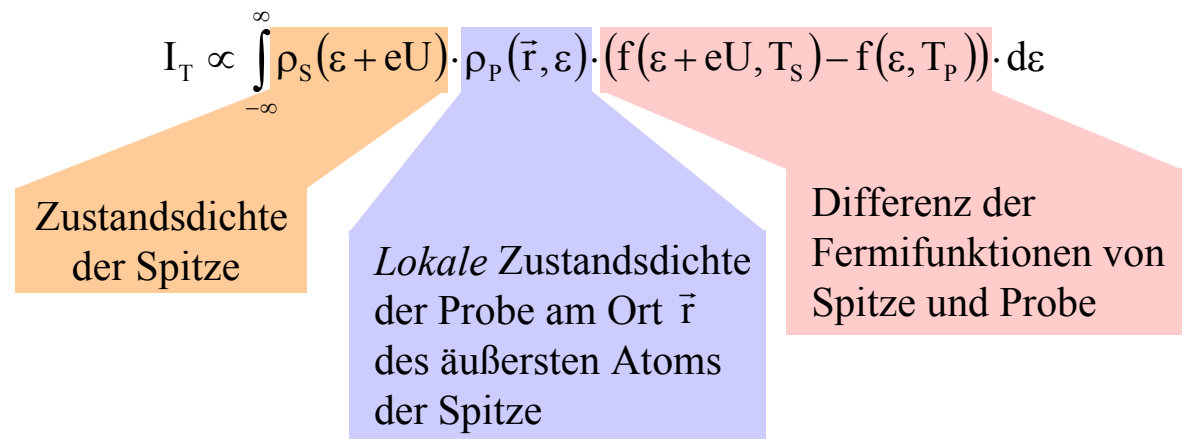

\subsection{Messmodi}

\subsubsection{Topografiemodus}

Der Topografiemodus wird auch „,constant-current-Modus“ genannt. Es wird eine konstante Spannung U zwischen Spitze und Probe angelegt ${ }^{4}$ und ein Soll-Wert $\mathrm{I}_{\text {soll }}$ für den Tunnelstrom festgelegt. Während der Rasterung der Spitze in x- und y-Richtung wird der Tunnelstrom $\mathrm{I}_{\mathrm{T}}$ erfasst und mit dem Sollwert verglichen. Bei Unterschreitung des Sollwertes wird die Spitze in z-Richtung näher an die Probe herangeführt bzw. bei Überschreitung entsprechend zurückgezogen. An jedem Punkt $(\mathrm{x}, \mathrm{y})$ wird für $\mathrm{I}_{\mathrm{T}}=\mathrm{I}_{\text {soll }}$ die Auslenkung $\mathrm{z}$ des Piezostellelementes erfasst. Die „Topografie“ ist also als eine Fläche konstanten Tunnelstromes für eine festgehaltene Spannung $U$ definiert.

Zur Veranschaulichung des Begriffes „Topografie“ werde ein Beispiel angenommen, bei dem $|\mathrm{eU}| » \mathrm{k}_{\mathrm{B}} \mathrm{T}$ gelte und die Zustandsdichte der Spitze als konstant angenommen wird. Das Tersoff-Hamann Integral vereinfacht sich dann zu

$$
\mathrm{I}_{\mathrm{T}} \propto \int_{0}^{\mathrm{eU}} \rho_{\mathrm{P}}(\overrightarrow{\mathrm{r}}, \varepsilon) \cdot \mathrm{d} \varepsilon
$$

\footnotetext{
${ }^{4}$ Im folgenden seien $U_{\text {Spitze }}$ bzw. $U_{\text {Probe }}$ die Spannungspotenziale von Spitze und Probe. Die Tunnelspannung sei $\mathrm{U}=\mathrm{U}_{\text {Spitze }}-\mathrm{U}_{\text {Probe }}$. Bei einem positiven Tunnelstrom $\mathrm{I}_{\mathrm{T}}$ tunneln Elektronen von der Spitze in die Probe.
} 
In diesem Fall folgt die Spitze für $\mathrm{I}_{\mathrm{T}}=\mathrm{I}_{\text {soll }}$ einer Fläche konstanter integrierter lokaler Zustandsdichte im Energiebereich zwischen $\varepsilon=0$ und $\varepsilon=\mathrm{eU}$.

\subsection{2. dI/dU-Spektroskopie}

Bei der dI/dU-Spektroskopie wird an einer festgehaltenen Position der Spitze die Tunnelspannung U um einen Betrag dU variiert und die Änderung dI im Strom gemessen. Für kleine Spannungsvariationen dU und bei einer als konstant angenommenen Zustandsdichte der Spitze kann gezeigt werden [Lan 1, Che 3], dass

$$
\left.\frac{\mathrm{dI}}{\mathrm{dU}} \propto \rho_{\mathrm{P}}(\overrightarrow{\mathrm{r}}, \varepsilon)\right|_{\varepsilon=\mathrm{eU}} \text {. }
$$

dI/dU-Spektroskopie erlaubt also einen direkten Zugang zu der lokalen Zustandsdichte der Probe bei der Energie $\varepsilon=\mathrm{eU}$.

\subsubsection{Potenziometrie}

Bei der Rastertunnel-Potenziometrie wird an einer festgehaltenen Position der Spitze das Spannungspotenzial $U_{\text {Pot }}$ der Spitze auf den Wert eingestellt, bei dem der Tunnelstrom zu $\mathrm{I}_{\mathrm{T}}=0$ wird. Erfasst wird die Größe $\left.\mathrm{U}_{\mathrm{Pot}}(\mathrm{x}, \mathrm{y})\right|_{\mathrm{I}_{\mathrm{T}}=0}$.

Bei einer Temperaturdifferenz zwischen Spitze und Probe wird $U_{\text {Pot }}(\mathrm{x}, \mathrm{y})$ (abzüglich einer Konstanten马, als lokale „Thermospannung“ des Tunnelkontaktes interpretiert.

\footnotetext{
${ }^{5}$ Da sowohl Probe wie auch Spitze eine andere Temperatur als die Ansteuerungselektronik besitzen, setzt $U_{\text {Pot }}$ sich aus der Thermospannung des Tunnelkontaktes sowie den konstanten Thermospannungsanteilen der $\mathrm{Zu}-$ leitungen zusammen.
} 


\section{Raster-Thermospannungs-Mikroskopie}

\subsection{Thermoelektrischer Effekt}

Wird ein elektrisch geschlossener Kreis aus zwei unterschiedlichen Metallen an einer der beiden Kontaktstellen erhitzt, dann fließt in diesem ein Kreisstrom. Dieser Effekt wurde bereits 1821 von T. J. Seebeck entdeckt und intensiv untersucht [See 1-2, Bec, Yel]. Die treibende Kraft für den diffusiven Ladungstransport ist das Temperaturgefälle entlang der elektrischen Leiter.

Bei Auftrennung des Kreises kann kein resultierender Strom mehr fließen. In diesem Fall wird durch entsprechende Ladungsanhäufung ein elektrisches Gegenfeld (das sogenannte Thomson-Feld) erzeugt, welches der thermischen Diffusion der Elektronen entgegenwirkt. Das Thomson-Feld ergibt entlang des gesamten Stromkreises integriert die Thermospannung. Dieses Prinzip wird beim Thermoelement ausgenutzt, bei dem die Thermospannung an der Trennstelle abgegriffen wird.

Eine zur Beschreibung thermoelektrischer Effekte wichtige Größe ist die Thermokraft S (auch Seebeckkoeffizient genannt), welche das Thomson-Feld $\overrightarrow{\mathrm{E}}_{\mathrm{Th}}$ linear mit dem Temperaturgradienten $\vec{\nabla} \mathrm{T}$ verknüpft [Bar 2].

$$
\overrightarrow{\mathrm{E}}_{\mathrm{Th}}=\mathrm{S}(\mathrm{T}) \cdot \vec{\nabla} \mathrm{T}
$$

Wenn S für hinreichend kleine Temperaturdifferenzen $\Delta \mathrm{T}$ als unabhängig von der Temperatur $\mathrm{T}$ angenommen werden kann, dann gilt die Beziehung

$$
\Delta \mathrm{U}_{\mathrm{Th}} \approx \mathrm{S} \cdot \Delta \mathrm{T}
$$

Die Thermokraft für Metalle unter der Annahme einer Fermiverteilung wurde erstmals von N. F. Mott hergeleitet [Mot] Demnach gilt bei einer spezifischen elektrischen Leitfähigkeit $\sigma_{\mathrm{e}}$ an der Fermikante bei $\varepsilon=0$ :

$$
\mathrm{S}(\mathrm{T})=-\left.\frac{\pi^{2} \cdot \mathrm{k}_{\mathrm{B}}^{2} \cdot \mathrm{T}}{3 \cdot \mathrm{e}} \cdot\left(\frac{1}{\sigma_{\mathrm{e}}} \cdot \frac{\mathrm{d} \sigma_{\mathrm{e}}}{\mathrm{d} \varepsilon}\right)\right|_{\varepsilon=0}
$$




\title{
3.2. Thermoelektrischer Effekt am Tunnelkontakt
}

Die Eigenschaften eines thermoelektrischen Kreises ändern sich entscheidend, wenn eine Tunnelbarriere vorhanden ist, welche nur einen Bruchteil der thermischen und elektrischen Leitfähigkeit relativ zu den Metallen besitzt. Für diesen Fall ist über die Barriere hinweg ein Sprung in der Temperatur zu erwarten. Am Tunnelkontakt liegt eine „Thermospannung“ an, welche im Experiment sehr empfindlich von der Beschaffenheit des Kontaktes abhängt.

So kommt es, dass selbst in einmetallischen thermoelektrischen Kreisen eine Thermospannung gemessen wird. Diesen Effekt erwähnten Seebeck und v. Yelin schon im Jahre 1821 [See 1-2, Yel]; in diesen Experimenten trat die Tunnelbarriere unbeabsichtigt in Form von oxidierten Metall-Kontaktierungen auf.

Eine Beschreibung der Thermospannung an einer Tunnelbarriere, welche die Fermiverteilung erstmals berücksichtigte, wurde 1940 von M. Kohler gegeben [Koh]. Eine experimentelle Untersuchung und Deutung des thermoelektrischen Effektes an einem Punktkontakt im Vakuum wurde erstmals von O. I. Shklyarevskii und Mitarbeitern [Shk] durchgeführt.

Neuere Theorien behandeln den thermoelektrischen Effekt im Tunnelkontakt eines STM [Lea, Sto.

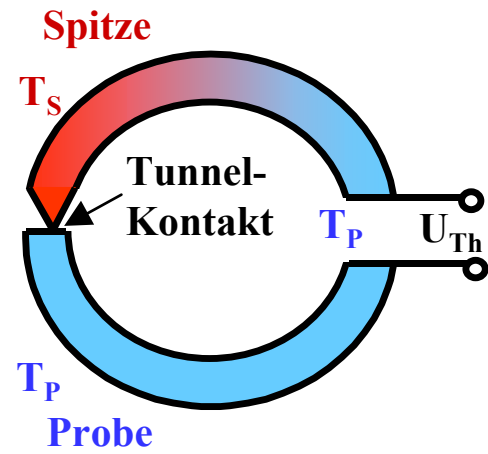

\begin{abstract}
Abb. 3.1 Thermoelektrischer Effekt am Tunnelkontakt eines STM. Die Spitze wird auf eine Temperatur $\mathrm{T}_{\mathrm{S}}$ erwärmt, die Probe besitzt eine Tempe$\operatorname{ratur} \mathrm{T}_{\mathrm{P}}<\mathrm{T}_{\mathrm{S}}$.
\end{abstract}

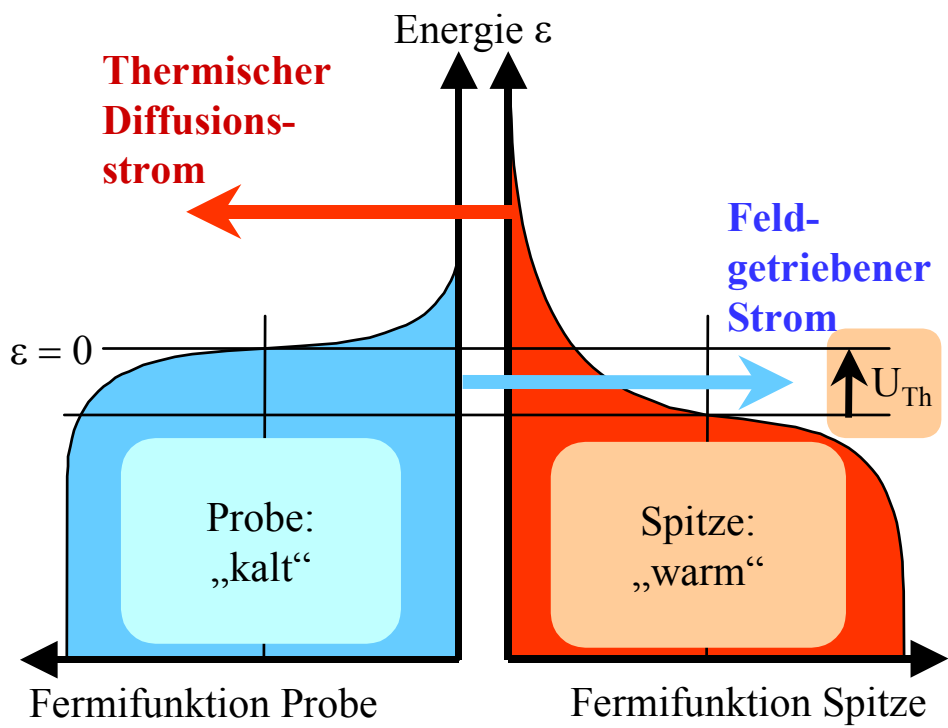

Abb. 3.2 Schematische Darstellung der Elektronen-Diffusionsströme über den Tunnelkontakt. Die unterschiedlichen Fermifunktionen auf beiden Seiten verursachen einen thermischen Diffusionsstrom, welcher von einem feldgetriebenen Diffusionsstrom (bestimmt durch $\left.\mathrm{U}_{\mathrm{Th}}\right)$ ausgeglichen wird. Die Richtungen der Ströme sind hier willkürlich dargestellt (s. Text). 
Der thermoelektrische Effekt am Tunnelkontakt soll im folgenden ausführlicher untersucht werden. Betrachtet sei dazu in Abb. 3.1 ein Tunnelkontakt, bei dem die Spitze gegenüber der Probe auf eine Temperatur $\mathrm{T}_{\mathrm{S}}>\mathrm{T}_{\mathrm{P}}$ erwärmt wird.

Die Messung der Thermospannung $\mathrm{U}_{\mathrm{Th}}$ erfolge stromlos.

Auf der wärmeren Seite des Tunnelkontaktes (hier die Spitze) ist die Fermiverteilung stärker verbreitert als auf der kälteren Probenseite Abb. 3.2). Elektronen mit hoher Energie $(\varepsilon » 0)$ diffundieren deshalb überwiegend von der wärmeren zur kälteren Seite hin. Entsprechend diffundieren Elektronen niedrigerer Energie $(\varepsilon \ll 0)$ bevorzugt in die umgekehrte Richtung.

Die Stärke der Diffusionsströme hängt von der „Leitfähigkeit“ $\sigma_{\mathrm{T}}(\varepsilon)$ der Tunnelbarriere ab. Für eine konstante Leitfähigkeit $\sigma_{\mathrm{T}}(\varepsilon)$ wären die thermisch aktivierten Diffusionsströme in beide Richtungen gleich groß und würden sich gegenseitig aufheben. Wenn die Leitfähigkeit $\sigma_{\mathrm{T}}(\varepsilon)$ aber abhängig von der Energie ist, dann kann entweder der Diffusionsstrom von „,heißen“ oder der von „,kalten“ Elektronen stärker gewichtet werden.

Das Vorzeichen von $\mathrm{d}_{\mathrm{T}} / \mathrm{d} \varepsilon$ bestimmt darüber, in welche Richtung der resultierende thermisch aktivierte Diffusionsstrom fließt (In Abb. 3.2 wurde beispielsweise $\mathrm{d} \sigma_{\mathrm{T}} / \mathrm{d} \varepsilon>0$ angenommen).

Ist der thermoelektrische Kreis wie in Abb. 3.1 gezeigt offen (also stromlos), dann muss der thermisch aktivierte Diffusionsstrom mit Hilfe eines Thomson-Gegenfeldes kompensiert werden. Dieses ist in Form einer Thermospannung $U_{T h}$ über den Tunnelkontakt zu finden.

Der Diffusionsstrom über die Tunnelbarriere ist äquivalent behandelbar wie der Diffusionsstrom innerhalb von Metallen, wie er von Mott beschrieben wurde [Mot]. Obige Beschreibung liefert somit eine anschauliche Erklärung für den $\mathrm{d} \sigma_{\mathrm{T}} / \mathrm{d} \varepsilon$-Term in der Formel von Mott (Gl. 3.3)

Demnach kann auch dem Tunnelkontakt formal eine „Thermokraft“ $\mathrm{S}=\mathrm{U}_{\mathrm{Th}} / \Delta \mathrm{T}$ zugeordnet werden. Dies wurde 1990 von J. A. Støvneng und P. Lipavský in einer theoretischen Arbeit untersucht [Sto], bei welcher der Tunnelkontakt im Modell von Tersoff und Hamann [Ter 1-3] behandelt wurde (Abb. 3.3), Als Näherung wurde angenommen, dass die Zustandsdichten im Bereich der thermischen Verbreiterung der Fermifunktionen als linear in der Energie angesehen werden können. 


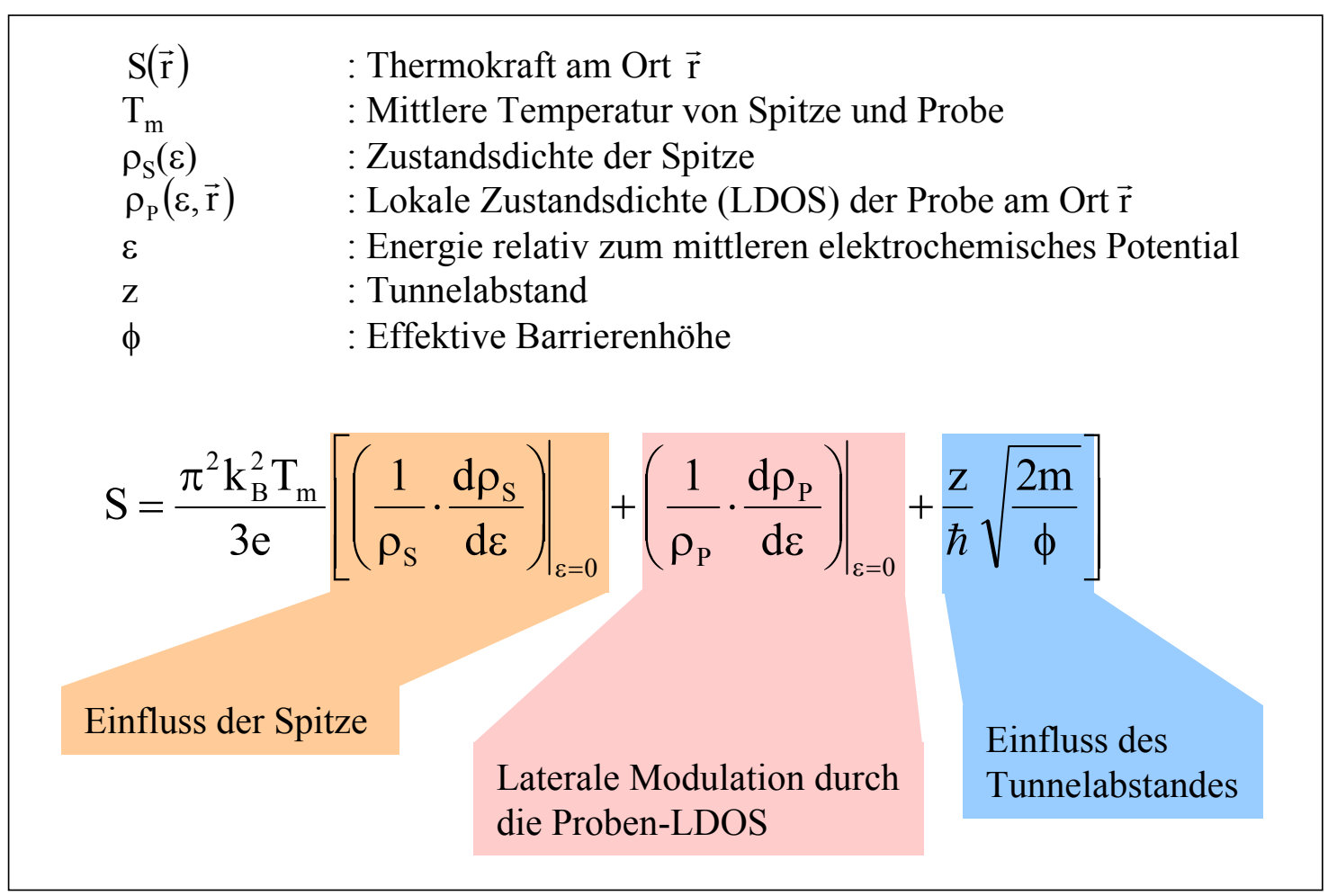

Abb. 3.3 Thermokraft eines Tunnelkontaktes nach J. A. Støvneng und P. Lipavský [Sto]

Es zeigt sich, dass letztere auf Basis von Tersoff und Hamann hergeleitete Gleichung identisch zur Mott- Formel (Gl. 3.3) ist, wenn dort die materialspezifische Leitfähigkeit $\sigma_{\mathrm{e}}$ ersetzt wird durch die Leitfähigkeit $\sigma_{\mathrm{T}}$ des Tunnelkontaktes, welche angesetzt wird zu

$$
\sigma_{\mathrm{T}}(\varepsilon) \propto \rho_{\mathrm{S}}(\varepsilon) \cdot \rho_{\mathrm{P}}(\varepsilon, \overrightarrow{\mathrm{r}})
$$

Die Thermokraft des Tunnelkontaktes besteht aus drei Termen. Interessant ist hier vor allem der mittlere Term in Abb. 3.3, welcher die Thermokraft von der lokalen Zustandsdichte der Probe abhängig macht.

Die Thermokraft $S$ ist demnach ortsabhängig und variiert mit der Ableitung der LDOS nach der Energie, $\mathrm{d} \rho_{\mathrm{P}} / \mathrm{d} \varepsilon$, bei $\varepsilon=0$.

Die beiden anderen Terme berücksichtigen jeweils die Konfiguration der Spitze sowie den Tunnelabstand und liefern im normalen STM-Betrieb einen nahezu konstanten Anteil. 


\subsection{Erstellung von Thermospannungs-Karten mit $\operatorname{dem}$ STM}

Wenn die Spitze eines STM Tunnelkontaktes gegenüber der Probe erwärmt wird, dann kann im Potenziometriemodus die Thermospannung $\mathrm{U}_{\mathrm{Th}}(\mathrm{x}, \mathrm{y})$ simultan zur Topografie ortsaufgelöst aufgezeichnet werden. Dieses Verfahren wird im folgenden „RasterThermospannungs-Mikroskopie“ (engl. Scanning-Thermovoltage-

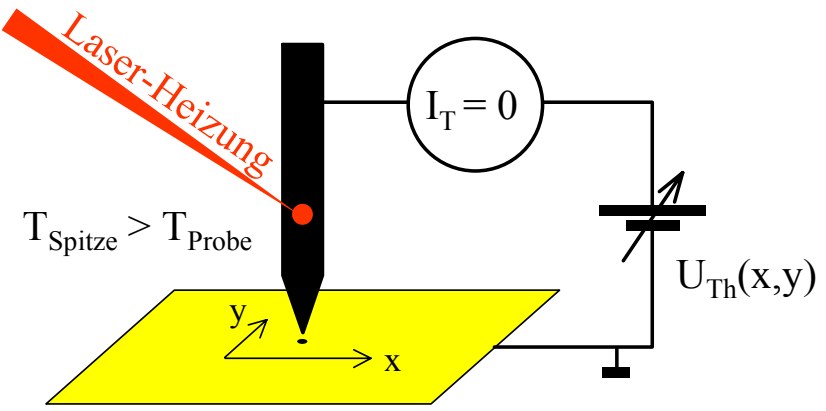

Abb. 3.4 „Raster-Thermospannungs-Mikroskopie“: Erstellung von Karten der Thermospannung über den Tunnelkontakt.

Microscopy, SThVM) genannt?

Bei einer Temperaturdifferenz $\Delta \mathrm{T}$ ist die Thermospannung $\mathrm{U}_{\mathrm{Th}}=\mathrm{S} \cdot \Delta \mathrm{T}$ direkt mit der Thermokraft S des Tunnelkontaktes verknüpft. Wenn die Darstellung für S in Abb. 3.3 auf das Wesentliche reduziert wird, dann ist in den „Thermospannungs-Karten“ vor allem die räumliche Variation $\Delta \mathrm{U}_{\mathrm{Th}}(\mathrm{x}, \mathrm{y})$ der Thermospannung interessant, denn es gilt 7

$$
\left.\Delta U_{\mathrm{Th}}(\mathrm{x}, \mathrm{y}) \propto \frac{\mathrm{d} \rho_{\mathrm{P}}(\varepsilon, \mathrm{x}, \mathrm{y}, \mathrm{z})}{\mathrm{d} \varepsilon}\right|_{\substack{\varepsilon=0 \\ \mathrm{z}=\mathrm{z}(\mathrm{x}, \mathrm{y})}}=: \rho_{\mathrm{P}}^{\prime}(\mathrm{x}, \mathrm{y}) .
$$

Aus den Thermospannungskarten kann somit direkt die Ableitung der lokalen Zustandsdichte nach der Energie, $\rho_{\mathrm{P}}^{\prime}(\mathrm{x}, \mathrm{y})$, bei $\varepsilon=0$ bestimmt werden.

SThVM ermöglicht damit die Messung einer physikalischen Größe, welche mit den üblicherweise angewandten STM-Techniken nicht leicht zugänglich ist. Wie in Kap. 2.3 beschrieben ist, wird in der Topografie eine integrale LDOS und bei der dI/dU-Spektroskopie direkt die LDOS gemessen.

Gegenüber dI/dU-Spektroskopie besitzt SThVM zwar den Nachteil, dass die LDOS nur für $\varepsilon=0$ untersucht werden kann, bietet aber den Vorteil einer höheren Empfindlichkeit auf relative Änderungen der LDOS.

\footnotetext{
${ }^{6}$ In der Literatur gibt es bisher noch keine einheitliche Benennung für dieses Verfahren.

${ }^{7}$ Diese Näherung gilt, wenn die lokale Zustandsdichte $\rho_{\mathrm{P}}(\varepsilon)$ innerhalb einer thermischen Verbreiterung von ca. $\pm 4 \mathrm{k}_{\mathrm{B}} T$ als näherungsweise linear um $\varepsilon=0$ angenommen werden kann.
} 
Eine ortsabhängige Thermospannung mit atomarer Auflösung wurde erstmalig von J. M. R. Weaver [Wea] und C. C. Williams [Wil 1-2] in der Gruppe um H. K. Wickramasinghe an geheizten Gold- und $\mathrm{MoS}_{2^{-}}$Substraten nachgewiesen. Seitdem kam dieses Verfahren in vielfältiger Weise zum Einsatz [Eng 2, Hof 2-6, Non, Ret, Sch 1-2, Sei, Xu 1].

In dieser Arbeit wird SThVM bei Temperaturen um $80 \mathrm{~K}$ bis $110 \mathrm{~K}$ verwendet, um stehende Wellen von interferierenden Elektronenwellen auf einer weitaus größeren räumlichen Skala $\mathrm{zu}$ detektieren und $\mathrm{zu}$ analysieren, als es bisher mit anderen topografischen oder spektroskopischen Methoden möglich war. 


\section{Experimenteller Aufbau}

\subsection{Apparatives}

\subsubsection{UHV-Tieftemperatur-STM}

Das zentrale Untersuchungsinstrument in dieser Arbeit ist das in unserer Gruppe entwickelte und von M. A. Rosentreter aufgebaute Ultrahochvakuum-Tieftemperatur-Rastertunnelmikroskop [Ros], welches optischen Zugang, „Cross-Section-STM“ und einen UHV Spitzen- und Probenwechsel ermöglicht.

Der prinzipielle Aufbau des Kryostaten ist in Abb. 4.1, der des Mikroskops ist in Abb. 4.2 dargestellt. Der Kryostat besteht aus zwei Tanksystemen. Der äußere Tank (grün in Abb. 4.1) enthält flüssigen Stickstoff $\left(\mathrm{LN}_{2}\right)$ und dient zur Kühlung der Kälteschilde, welche den inneren Tank komplett vor der Raumtemperatur-Wärmestrahlung abschirmen. Der innere Tank kann wahlweise mit $\mathrm{LN}_{2}$ oder flüssigem Helium (LHe) gefüllt werden. Das STM befindet sich in einem vergoldeten Kupfertopf, welcher direkt unter dem inneren Tank angeschraubt ist. Zur besseren thermischen Kopplung befindet sich zwischen dem Topf und dem inneren Tank eine Indiumdichtung. Bei Füllung mit $\mathrm{LN}_{2}$ werden am STM-Sockel mit einer Siliziumdiode Temperaturen von $79 \mathrm{~K}$ gemessen.

Das STM besitzt einen optischen Zugang, über welchen die Spitze mit Hilfe eines Lasers erwärmt werden kann. Die Öffnungen im LN2-Schild sind mit thermisch gut angekoppelten Quarzgläsern verschlossen, welche durch Adsorption von Infrarot-Strahlung den inneren Tank vor Raumtemperatur-Wärmestrahlung schützen.

Zur Erzeugung des Ultrahochvakuums (UHV) wird eine Ionenzerstäuberpumpe benutzt, welche von einem Titanverdampfer unterstützt wird. Der außerhalb der Kältetanks gemessene Druck liegt üblicherweise zwischen $7 \cdot 10^{-11}$ mbar und $2 \cdot 10^{-10}$ mbar.

Die Schwingungsdämpfung für kleine Frequenzen erfolgt einerseits dadurch, dass die gesamte Anlage auf luftgepolsterte Füße gestellt wird. Andererseits besitzt der Innentank eine zusätzliche Dämpfung durch seinen pendelartigen Aufbau, bei dem er von der restlichen Kammer durch eine Kombination von Well- und Membranbalg entkoppelt ist. Die Eigenfrequenzen dieses Aufbaus entsprechen $3 \mathrm{~Hz}$ in die horizontale Richtung und $45 \mathrm{~Hz}$ in die vertikale Richtung, so dass Bewegungen der Kammer oberhalb dieser Frequenzen effektiv abgeschirmt werden. Frequenzen oberhalb von ca. $500 \mathrm{~Hz}$ werden durch eine VitonDämpfung des Wellbalgs mit einem Tiefpass der Ordnung 3.5 abgeschirmt [Ros]. 


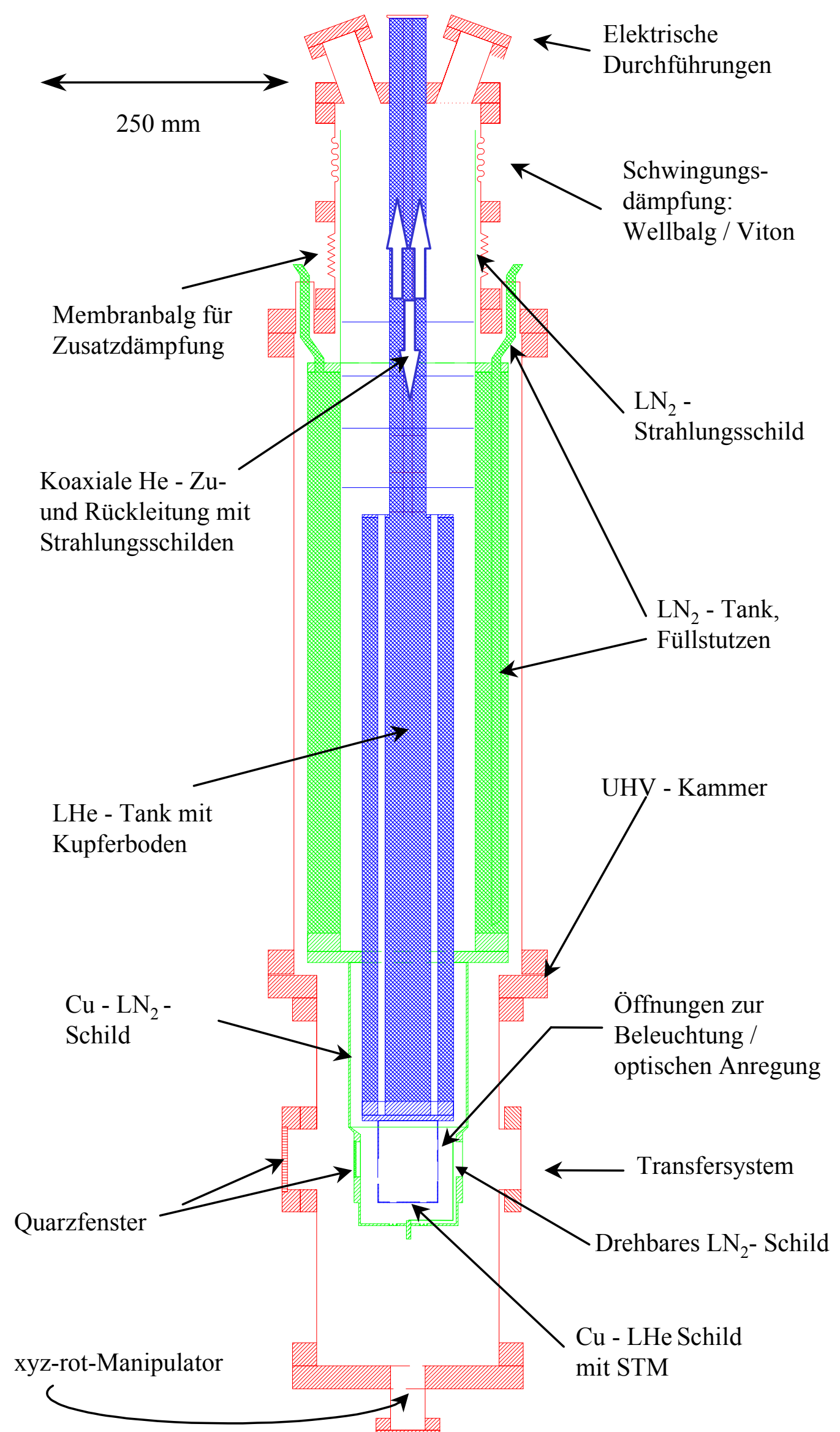

Abb. 4.1 Schema des UHV-Tieftemperatur STM (nach M. A. Rosentreter [Ros]] 


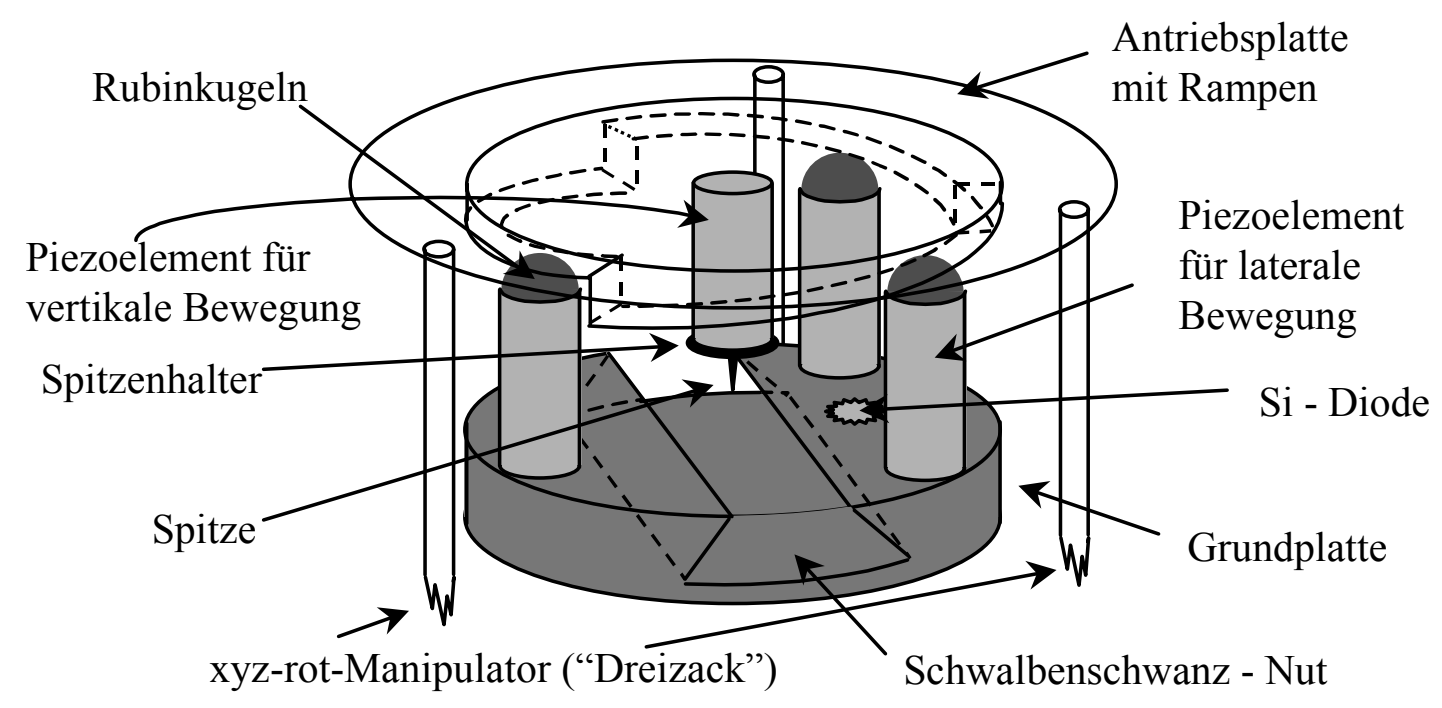

Abb. 4.2 Schema des STM (nach M. A. Rosentreter [Ros]

Das STM ist vom sogenannten „Beetle“-Typ, dessen Prinzip 1987 von K. H. Besocke entwickelt wurde [Bes 2-3]. In unserer Arbeitsgruppe wurde es von T. Quast konzipiert und aufgebaut [Qua]. In diesem Design erlaubt das STM eine zielgenaue laterale Positionierung der Spitze innerhalb eines Umkreises von ca. $6 \mathrm{~mm}$ Durchmesser auf der Probe (bekannt unter dem Begriff „Cross-Section STM“). Es ermöglicht unter UHV-Bedingungen einen Wechsel von Spitze oder Probe und gewährleistet eine relativ schnelle und unproblematische Fein-Annäherung der Spitze an die Probe. Zudem bietet der weitgehend symmetrische Aufbau aus identischen Materialien eine relativ gute Stabilität gegenüber thermischer Drift.

Das Piezostellelement für die Steuerung des Tunnelabstandes (die ,z-Richtung“) ist zentral unter einer Platte montiert, welche auf Rubinkugeln auf drei für die laterale Bewegung (in xund y-Richtung) zuständigen Piezostellelementen aufliegt.

Zum Wechseln der magnetisch gehalterten Spitze oder zum groben Positionieren kann diese Platte mit einem an einen Manipulator befestigten „Dreizack“ angehoben, in alle Raumrichtungen bewegt sowie um die z-Achse rotiert werden.

Alternativ $\mathrm{zu}$ der groben Positionierung kann der Plattenteller auch mit Hilfe der Piezostellelemente lateral bewegt werden. Hierzu werden alle Piezostellelemente wiederholt zunächst langsam in die gewünschte Richtung bewegt und dann ruckartig zurückgezogen (bekannt als „Slip-Stick-Verfahren“). Durch die Massenträgheit führt der Plattenteller den ruckartigen zweiten Schritt nicht mit aus und bewegt sich effektiv vorwärts. Auf diese Weise ist es auch möglich, in situ nur durch einfaches Zurückziehen der Spitze aus dem Tunnelkontakt und anschließendem „Slip-Stick“ große Bereiche der Probenoberfläche systematisch zu untersuchen.

Für die Feinannäherung der Spitze sind an der Unterseite der Platte drei kreisförmig zusammengeschlossene Rampen untergebracht, welche direkt auf den Rubinkugeln gelagert sind. Wenn die Platte um die z-Achse gedreht wird, dann bewirken die Rampen je nach 
Drehrichtung ein Heben oder Senken der gesamten Platte mit einem möglichen Gesamthub von ca. $1.2 \mathrm{~mm}$. Die notwendige Drehbewegung des Plattentellers wird von den drei äußeren Piezostellelementen im Slip-Stick-Verfahren übernommen.

Die Probenkontaktierung geschieht durch zwei (in Abb. 4.2 nicht dargestellten) Federblechen an den Enden der Schwalbenschwanz-Nut. Aus Stabilitätsgründen und zur besseren thermischen Ankopplung wird die Probe mit Hilfe eines an der Unterseite der Nut angebrachten Federbleches gegen die angeschrägten Seitenflächen der Nut gedrückt.

Der lateral mögliche Rasterbereich der drei äußeren Piezos beträgt bei Raumtemperatur ca. $(10 \times 10) \mu \mathrm{m}^{2}$, nimmt aber bei $\mathrm{LN}_{2}$-Kühlung aufgrund des Empfindlichkeitsverlustes der Piezostellelemente auf etwa $(6 \times 6) \mu \mathrm{m}^{2} \mathrm{ab}$. Der entsprechende Hub des z-Piezostellelementes beträgt etwa $1.8 \mu \mathrm{m}$ bei Raumtemperatur und $1 \mu \mathrm{m}$ bei $\mathrm{LN}_{2}$-Kühlung.

\subsubsection{UHV-Peripherie}

Die Präparationsanlage für Spitzen und Proben ist räumlich getrennt vom TieftemperaturSTM untergebracht. Ein Transport von Spitzen und Proben wird mit einem fahrbaren UHVSystem vorgenommen, welches über Schleusen sowohl an die Präparationskammer wie auch an die STM-Kammer angekoppelt werden kann. Auf diese Weise ist es möglich, sowohl Spitzen wie auch Proben von der Präparation bis zur Untersuchung im STM unter UHVBedingungen zu lagern.

\section{Sputterquelle:}

In der Präparationskammer befindet sich die Sputterquelle IQE 11/35 der Firma Specs [Spe]. Die Quelle besitzt ein gaußsches Strahlprofil mit einer Halbwertsbreite von $2 \mathrm{~cm}$ an der Probenposition und kann Ionenströme bis zu $10 \mu \mathrm{A}$ bei einer maximalen Ionenenergie von $5 \mathrm{keV}$ erzeugen. Sowohl zur Präparation von Spitzen wie auch von Proben werden positiv geladene Argon-Ionen verwendet.

\section{Heizapparatur:}

Proben können mit einer in Eigenbau entwickelten Heizapparatur bis zu Temperaturen von ca. $900{ }^{\circ} \mathrm{C}$ geglüht werden. Die Heizung erfolgt mit einem Bornitrid-Heizelement Boralelectric ${ }^{\circledR}$ HT-03 der Firma Tectra [Tec] mitsamt zugehörigem Netzteil HC3500. Um ein Aufwärmen der Kammerwände zu vermindern, ist die Heizapparatur von Hitzeschilden aus Molybdänblech umgeben. 


\subsection{Messtechnik}

\subsubsection{Elektronik}

Die Steuerung des STM basiert auf der Kombination zweier Rechner, dem „Messrechner“ und dem „Regelrechner“ (Abb. 4.3). Dieses System wurde in unserer Gruppe von N. Theuerkauf aufgebaut und detailliert beschrieben [The].

Die zentrale Steuereinheit bildet der Messrechner, welcher den Ablauf einer Messung festlegt, die Messdaten erfasst sowie die Spitze in $\mathrm{x} / \mathrm{y}$-Richtung bewegt.

Im Regelrechner ist der digitale Regelkreis (PI-Regelung [Mer]) für die z-Ansteuerung der Spitze implementiert. In seinem Arbeitsmodus wird er vom Messrechner kontrolliert.

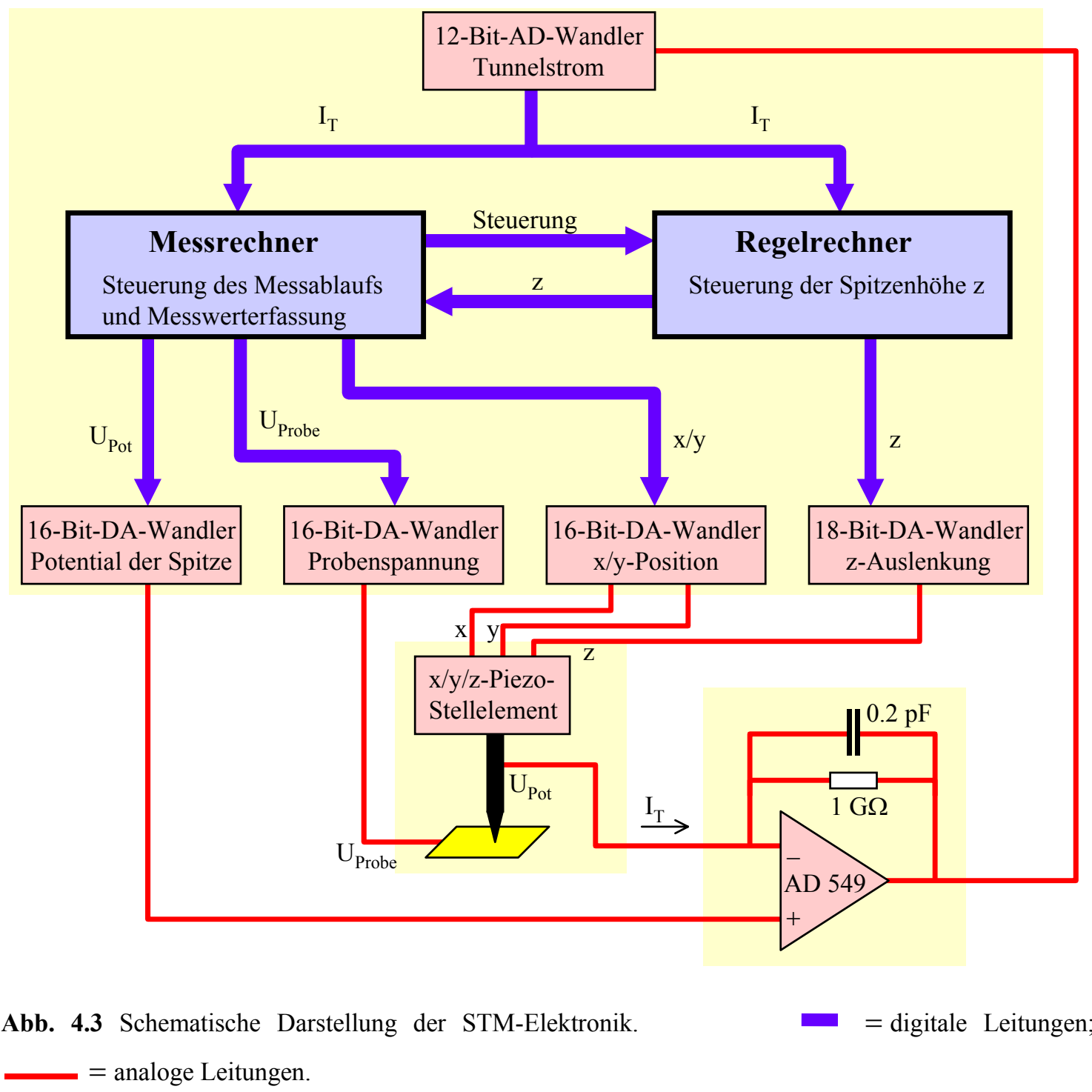

Der Tunnelstrom wird mit einem I/U-Konverter (AD 549, Faktor 1 V/nA) der Firma Analog Devices verstärkt. Von herkömmlichen Stromverstärkerbeschaltungen unterscheidet sich unsere darin, dass die Spitze auf ein vom Messrechner definiertes Potenzial $U_{\text {Pot }}$ gesetzt 
werden kann $\stackrel{8}{\square}$ Im Rückkopplungszweig des I/U-Konverters ist eine Kapazität von 0.2 pF eingesetzt, welche die Bandbreite auf $1 \mathrm{kHz}$ begrenzt. Eine detaillierte Charakterisierung des Stromverstärkers ist in Ref. [Eng 2] zu finden.

\subsubsection{Potenziometrie}

Das hier beschriebene Potenziometrieverfahren wurde in unserer Gruppe von M. A. Schneider entwickelt und detailliert beschrieben [Sch 1], so dass an dieser Stelle nur auf die wesentlichen Punkte eingegangen werden soll.

Im Potenziometriemodus wird an jedem Messpunkt (x,y) zunächst eine Abstandsregelung und sodann der Potenzialabgleich der Spitze auf einen verschwindenden Tunnelstrom vorgenommen.

\section{Die Abstandsregelung:}

- Die Spitze wird zum nächsten Messpunkt (x,y) bewegt. Die Abstandsregelung wird im constant-current-Modus durchgeführt und ein Messwert z(x,y) als „Topografie“ erfasst.

\section{Die Potenzialregelung:}

- Die Abstandsregelung wird unterbrochen und die Position der Spitze festgehalten.

- Das Potenzial $U_{\text {Probe }}$ der Probe wird auf 0 V gesetzt.

- Das Potenzial $U_{\text {Pot }}$ der Spitze wird mittels PI-Regelung [Mer] auf einen verschwindenden Tunnelstrom $\mathrm{I}_{\mathrm{T}}=0$ geregelt. Der Potenzialwert $\mathrm{U}_{\text {Pot }}(\mathrm{x}, \mathrm{y})$ wird erfasst. Bei thermoelektrischer Rastertunnelmikroskopie ist dieser Wert identisch mit der Thermospannung $\mathrm{U}_{\mathrm{Th}}(\mathrm{x}, \mathrm{y})$.

- Das Potenzial der Probe wird wieder auf den für die Abstandsregelung benötigten Wert $\mathrm{U}_{\text {Bias }}$ gesetzt.

- Die Abstandregelung der Spitze wird wieder aufgenommen.

Die experimentell gemessenen Werte von $U_{\text {Pot }}$ für einen Tunnelkontakt bei $80 \mathrm{~K}$ auf $\mathrm{Au}(111)$ besitzen eine Standardabweichung von $0.7 \mu \mathrm{V}$ bei 40 ms Regelphasendauer (s. Abb. 4.4).

\footnotetext{
${ }^{8}$ Das Potenzial $U_{\text {Pot }}$ wird vom Messrechner auf den nichtinvertierenden Eingang „, ${ }^{\text {“ }}$ des Operationsverstärkers gelegt. Die hohe interne Verstärkung des Operationsverstärkers sorgt zusammen mit dem rückgekoppelten Ausgangssignal dafür, dass der mit der Spitze verbundene invertierende Eingang „-“ sich auf nahezu dem selben Potenzial $\mathrm{U}_{\text {Pot }}$ befindet.
} 


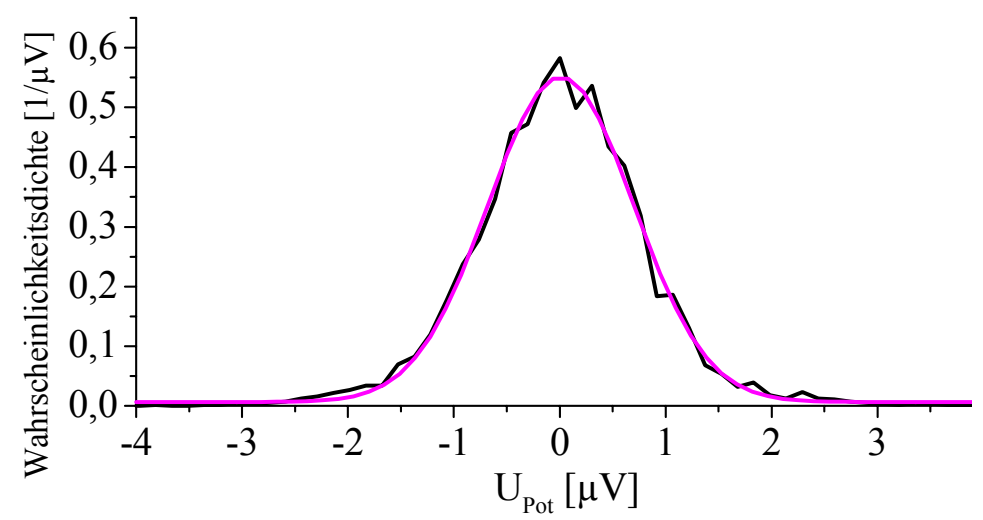

Abb. 4.4 Rauschen des Potenziometrieverfahrens im Tunnelbetrieb auf $\mathrm{Au}(111)$ bei $80 \mathrm{~K}$. Aufgetragen ist eine Verteilungsfunktion (—) für ca. 4000 Messwerte von $\mathrm{U}_{\text {Pot }}$ Verwendete Tunnelparameter: $\mathrm{U}_{\text {Bias }}=-1 \mathrm{mV}, \quad \mathrm{I}_{\mathrm{soll}}=1 \mathrm{nA}$. Eine angepasste Gaußkurve (_) ergibt eine Standardabweichung von $0.7 \mu \mathrm{V}$.

Die technische Realisierung ist eine Weiterentwicklung der ursprünglich von Muralt und Pohl entwickelten Rastertunnelpotenziometrie [Mur 1-2]. Diese und auch die in der Folgezeit entstandenen Techniken Kir 1-2, Pel Rei 2] können die Messvorschrift eines verschwindenden Tunnelstromes nicht erfüllen. Bei diesen Verfahren liegt eine Spannungsdifferenz zwischen Spitze und Probe an, da neben der Potenzialmessung auch noch der Abstand zwischen Probe und Spitze geregelt werden muss. Neben dem hier angewendeten Verfahren erfüllt nur das von R. Möller angegebene [Möl 3] die Messvorschrift $\mathrm{I}_{\mathrm{T}}=0 \mathrm{~A}$. Die Erfüllung dieser Bedingung besitzt entscheidende Vorteile:

- Die Störung der elektronischen Verteilungsfunktion in der Probe wird minimiert.

- Die Messgröße $U_{\text {Pot }}$ ist nahezu unabhängig vom Tunnelwiderstand.

- Das 1/f-Rauschen des Tunnelstromes $\mathrm{I}_{\mathrm{T}}$ verschwindet für $\left\langle\mathrm{I}_{\mathrm{T}}\right\rangle=0$ A Möl1-2] Übrig bleibt hauptsächlich das thermische weiße Rauschen des Tunnelwiderstandes ( Johnson/ Nyquist- Rauschen, [ Joh, Nyq, Buc] ).

Vor allem der letztere Punkt ist ausschlaggebend für die hohe erreichte Auflösung des Potenziometriesignals. Für $\left\langle\mathrm{I}_{\mathrm{T}}\right\rangle=0 \mathrm{~A}$ wird das technisch beobachtete Rauschen nur noch durch das Eingangs-Offsetrauschen des Stromverstärkers dominiert [Eng 2]

\subsection{Erzeugung von Temperaturdifferenzen}

\subsubsection{Temperaturmessung}

Die Probentemperatur kann über die im Sockel des STM angebrachte Siliziumdiode abgeschätzt werden. Die reale Temperatur liegt vermutlich aufgrund der nicht idealen Wärmeleitung der Probenhalterung um einige K höher. Temperaturerhöhungen der Spitze werden abgeschätzt, indem außerhalb des Vakuums in ähnlicher Anordnung die Spitze durch ein Thermoelement ersetzt und mit dem Laser bestrahlt wird [Eng 2]. Alternativ wird die Temperatur der Spitze durch deren Längenausdehnung bestimmt [Sch 1] Aus diesen Messungen wird eine mittlere Thermokraft $\mathrm{S}_{0}$ für den Tunnelkontakt berechnet, mit deren 
Hilfe in späteren Messungen auf den Temperaturunterschied zwischen Spitze und Probe zurückgeschlossen wird. In Tab. 4.1 ist die nach [Eng 2] bestimmte Thermokraft in Vergleich $\mathrm{zu}$ anderen experimentellen Ergebnissen tabelliert. Für auf diese Weise bestimmte Temperaturdifferenzen wird eine Unsicherheit von ca. 50\% angenommen.

\begin{tabular}{|l|l|l|l|l|}
\hline $\mathrm{S}_{0}$ in $\mu \mathrm{V} / \mathrm{K}$ & $\mathrm{T}_{\text {Probe }}$ & $\mathrm{T}_{\text {Spitze }}$ & $\begin{array}{l}\mathrm{S}_{0} \text { in } \mu \mathrm{V} / \mathrm{K}, \text { korrigiert für } \\
\mathrm{T}_{\mathrm{m}}=80 \mathrm{~K} ; \mathrm{T}_{\mathrm{m}}=100 \mathrm{~K}\end{array}$ & \\
\hline-12 & $293 \mathrm{~K}-303 \mathrm{~K}$ & $293 \mathrm{~K}$ & $-3.2 ;-4.0$ & \multicolumn{2}{|l|}{ [Eng 2] } \\
\hline-20 & $293 \mathrm{~K}$ & $298 \mathrm{~K}$ & $-5.4 ;-6.8$ & [Hof 2] \\
\hline-8.5 & $90 \mathrm{~K}$ & $290 \mathrm{~K}$ & $-3.6 ;-4.5$ & [Sei] \\
\hline
\end{tabular}

Tab. 4.1 Experimentell bestimmte Thermokräfte für die $\mathrm{Au}(111)$-Oberfläche

\subsubsection{Erwärmung der Spitze}

Die Erwärmung der Spitze bis zu $30 \mathrm{~K}$ erfolgt mittels Laserheizung über die optischen Zugänge des Kryostaten. Bei $\mathrm{LN}_{2}$-Kühlung stellen sich kleine Temperaturdifferenzen von ca. 2-3 K auch ohne Laserheizung aufgrund von Rest-Wärmestrahlung durch die Quarzgläser ein. Größere Temperaturunterschiede von bis zu einigen $10 \mathrm{~K}$ können erzeugt werden, indem die Verschlussklappe zur Transportöffnung für Spitzen und Proben geöffnet wird. Dieses Verfahren ist aber nicht zu empfehlen, da die gekühlten Proben relativ schnell AdsorbatBedeckungen zeigen.

\subsubsection{Wärmeleitung der Tunnelbarriere}

Es ergibt sich die Fragestellung, wie der Wärmetransport über den Tunnelkontakt stattfindet, und in wie weit dieser die Temperaturverteilung in unmittelbarer Nähe des Kontaktes beeinflusst. Eine ausführliche Untersuchung dieser Thematik wurde von J. B. Xu und Mitarbeitern geleistet [Xu 2-4], Die Mechanismen des Wärmetransports über den Tunnelkontakt sind demnach sehr speziell von der experimentellen Durchführung abhängig. Im einzelnen wurden untersucht:

- Wärmetransport über Elektronenaustausch [Xu 4].

- Wärmeaustausch über Restgasmoleküle oder Oberflächenadsorbate [Dra, Xu 2],

- Wärmestrahlung nach dem Stefan- Boltzmann- Gesetz [Dra, Xu 2$]$ und

- elektromagnetische Strahlung von Oberflächenplasmonen Dra $\mathrm{Xu} 2$.

Bei Tunnelkontakten an Luft überwiegt die Wärmeleitung über Adsorbatschichten und Wasserfilme [Wil 1-3, Wea, Maj, Oes 1-3]. Demgegenüber ist die Wärmeleitung von 
Tunnelkontakten im UHV bei sauberen Proben- und Spitzenoberflächen vernachlässigbar klein im Vergleich zur metallischen Wärmeleitung in Probe und Spitze [Xu 2-4].

\subsection{Präparatives}

Es werden zwei unterschiedliche Probentypen verwendet. Für die Messungen an Raumtemperatur werden zunächst epitaktisch auf Glimmer oder Glassubstrate aufgedampfte Goldfilme verwendet, während im späteren Stadium für die Messungen an tiefen Temperaturen ein $\mathrm{Au}(111)$ Einkristall benutzt wird. Beide Systeme besitzen spezifische Vorund Nachteile, welche für die jeweilige Art der Messung gegeneinander abgewogen werden müssen. Für diese Arbeit ist eine $\mathrm{Au}(111)$ Oberfläche gefordert, welche nicht nur einen hohen Reinheitsgrad, sondern auch relativ große, atomar ebene (111) Terrassen von mehreren $100 \mathrm{~nm}$ Durchmesser aufweisen sollte.

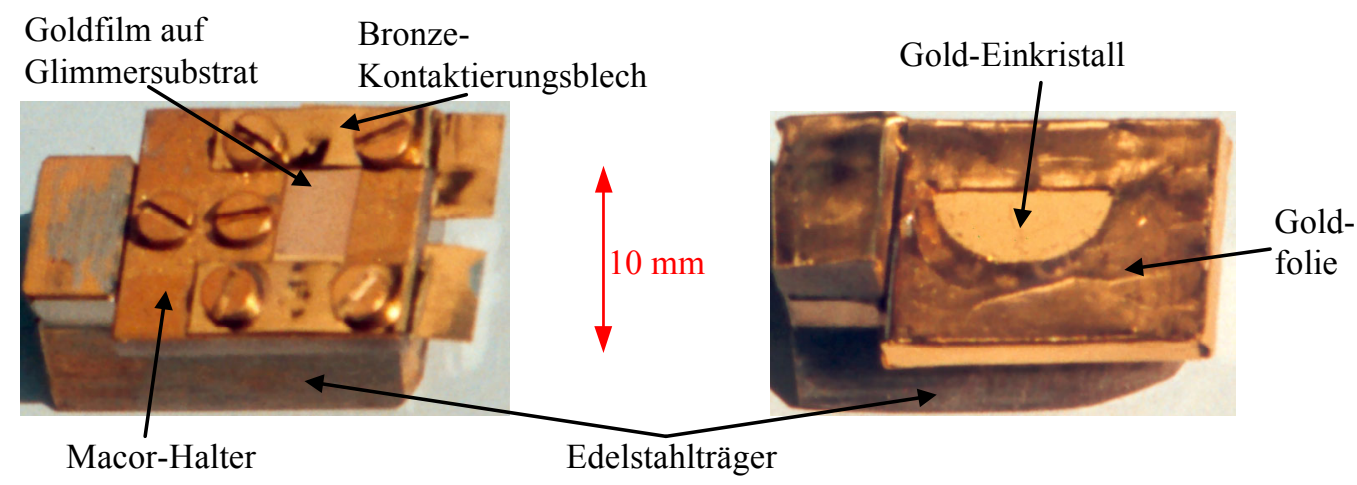

Abb. 4.5 Links: Probenhalter für aufgedampfte Goldfilme; rechts: Probenhalter für den Gold-Einkristall

\subsubsection{Epitaktisch hergestellte Goldfilme}

Aufgedampfte Goldfilme bieten den Vorteil, dass sie relativ einfach herzustellen sind. Zudem ist auch die Präparation in der Literatur schon gut dokumentiert [Pas, Qua]. Allerdings besitzen sie den Nachteil, dass nur relativ kleine atomar ebene Terrassen präpariert werden können. Je nach Wahl des Substratmaterials, der Schichtdicke und der Substrattemperatur können verschiedene Oberflächentopologien mit (111)-Textur hergestellt werden. Entsprechend dem Vollmer- Weber- Wachstumsmodell entstehen während des Aufdampfens zunächst kleine Inseln, welche sich durch Anlagerung von diffundierenden Atomen zunächst vergrößern und später dann zusammenwachsen Pas, Qua 


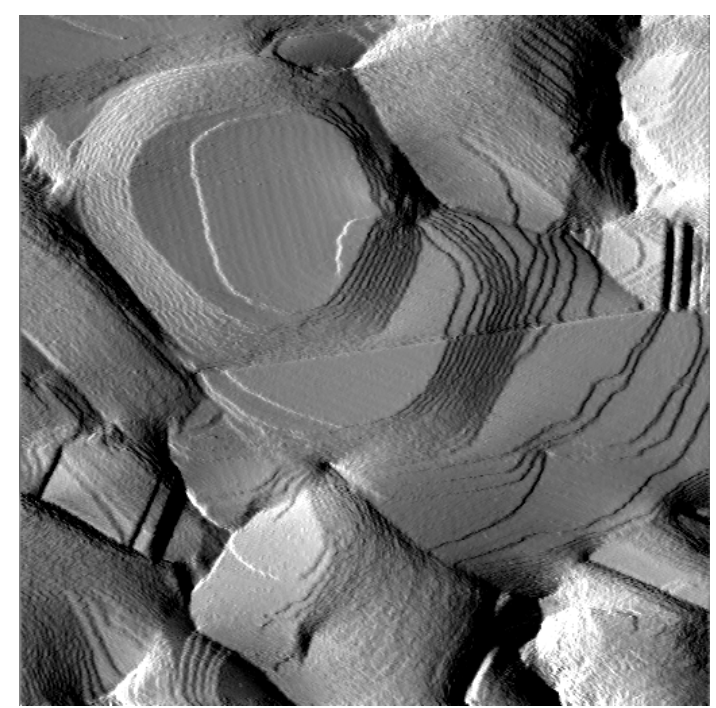

Abb. 4.6 Topografie eines bei $20^{\circ} \mathrm{C}$ aufgewachsenen Goldfilmes $\left(270 \times 270 \mathrm{~nm}^{2}\right)$ $0 \mathrm{~nm} \stackrel{\mathrm{nm}}{\square}$

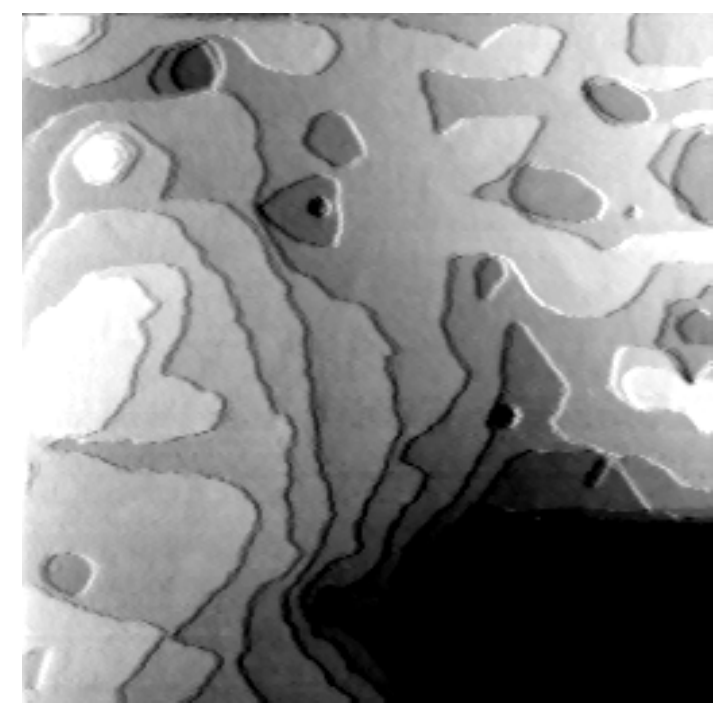

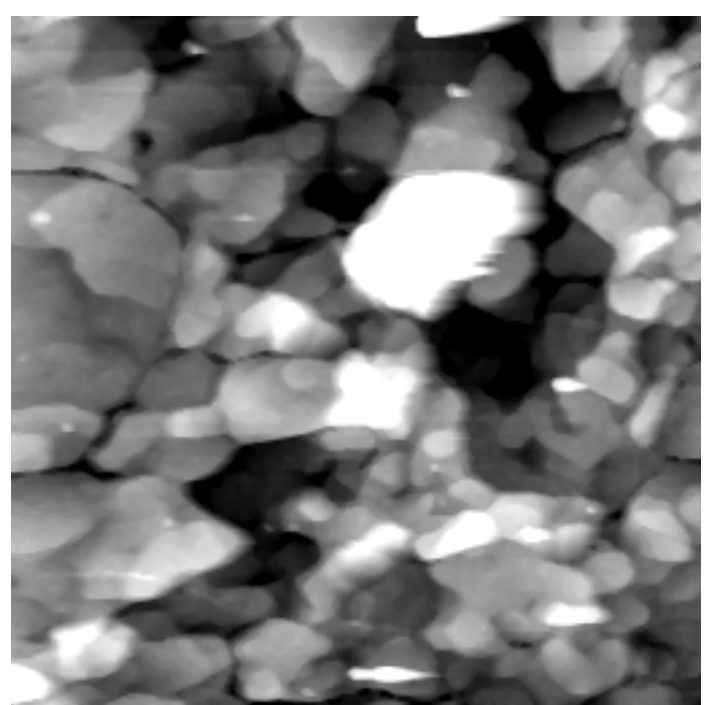

Abb. 4.7 Topografie eines bei $400^{\circ} \mathrm{C}$ aufgewachsenen Goldfilmes $\left(2500 \times 2500 \mathrm{~nm}^{2}\right)$

$0 \mathrm{~nm} \llbracket 20 \mathrm{~nm}$
Abb. 4.8 Topografie eines bei $400^{\circ} \mathrm{C}$ aufgewachsenen Goldfilmes $\left(250 \times 250 \mathrm{~nm}^{2}\right)$

$0 \mathrm{~nm} \square 2 \mathrm{~nm}$

Wenn die Substrattemperatur auf Raumtemperatur gehalten wird, können sehr saubere Oberflächen mit nur wenigen nm Rauhigkeit hergestellt werden (Abb. 4.6). Im Ergebnis erhält man jedoch bestenfalls Terrassenweiten, welche den Korndurchmessern zwischen 50 und $100 \mathrm{~nm}$ entsprechen. Wird bei höheren Substrattemperaturen (ca. $400^{\circ} \mathrm{C}$ ) aufgedampft, dann bilden sich Körner mit Durchmessern bis zu $1 \mu \mathrm{m}$, welche aber durch relativ tiefe Gräben (bis zu einigen $10 \mathrm{~nm}$ ) voneinander getrennt sind (Abb. 4.7). Aufgrund der leichten Rundungen der Körner werden auch bei dieser Art der Präparation keine nennenswert größeren atomar ebenen Terrassen als 100 bis $200 \mathrm{~nm}$ im Durchmesser gefunden (Abb. 4.8)

Die Goldfilme bei $400^{\circ} \mathrm{C}$ sind auf Glimmersubstrate aufgedampft, bei Raumtemperatur werden alternativ auch Glassubstrate benutzt. Zwischen Glas- und Glimmersubstraten werden keine wesentlichen Unterschiede in Korngröße und Topografie gefunden. Für die bei $400^{\circ} \mathrm{C}$ aufgedampften Proben werden die Glimmersubstrate zwecks Ausgasung zuvor etwa $12 \mathrm{~h}$ lang 
bei $400^{\circ} \mathrm{C}$ geglüht. Die hergestellten Filme besitzen eine Dicke von ca. $100 \mathrm{~nm}$ bei $400^{\circ} \mathrm{C}$ bzw. ca. $30 \mathrm{~nm}$ bei $20^{\circ} \mathrm{C}$ Substrattemperatur. Die Aufdampfrate beträgt jeweils $0.1 \mathrm{~nm} / \mathrm{s}$. Die Substrate mit den Abmessungen von $13 \mathrm{~mm} \times 4 \mathrm{~mm} \times 0.1 \mathrm{~mm}$ (Abb. 4.5) werden vor der eigentlichen Schichtpräparation an den beiden Längsenden mit Gold bedampft. Diese Randschichten dienen als elektrische Kontaktierung des Goldfilms, welcher später bei einem Basisdruck von besser als 5.0 $10^{-9}$ mbar auf die gesamte Substratfläche aufgedampft wird. Die Substratplättchen werden mit Bronzeblech auf Macor Haltern festgeklemmt; die Bronzebleche stellen außerdem die elektrische Kontaktierung des Filmes mit den Kontaktbügeln im STM her.

\subsubsection{Präparation des Au(111)-Einkristalls}

Bezüglich der Untersuchung von Interferenzeffekten sind epitaktisch hergestellte Filme nur begrenzt verwendbar. Das Interferenzmuster von Streuzentren (z.B. monatomaren Stufen) hängt sehr empfindlich auch von umgebenden Streuzentren $a b$, welche innerhalb von Abständen in der Größenordnung der Elektronenkohärenzlänge liegen. Für genügend kleine Terrassenweiten sind bereits Quantisierungseffekte aufgrund von Elektronen-Confinement beobachtet worden [SW 2] Um Effekte dieser Art weitestgehend zu vermeiden, wird eine $\mathrm{Au}(111)$-Oberfläche auf einem Einkristall präpariert.

Einkristalle bieten den Vorteil, dass mit ihnen atomar ebene Terrassen mit Ausdehnungen bis zu einigen $\mu \mathrm{m}$ präpariert werden können. Von Nachteil ist allerdings der höhere präparative Aufwand, der zumindest für die Vorpräparation betrieben werden muss.

Der in dieser Arbeit verwendete Gold Einkristall wurde von der Firma MaTeck [MaT] hergestellt. Er besteht aus einer halbierten Scheibe von $10 \mathrm{~mm}$ Durchmesser und $1.5 \mathrm{~mm}$ Höhe. Bei Lieferung war die Oberfläche mit einer Genauigkeit von $<0.1^{\circ}$ bezüglich (111) mit einer Rauhigkeit von $30 \mathrm{~nm}$ poliert. Die Reinheit des Kristalls beträgt $99.999 \%$ (Herstellerangabe).

Bevor dieser Kristall im STM untersucht werden kann, ist eine Befreiung der Oberfläche von sämtlichen Kontaminationen und eine Reduzierung der Rauhigkeit vonnöten. Dies geschieht in mehreren UHV- Vorpräparationsschritten, bei denen der Kristall wiederholt gesputtert und bei Temperaturen zwischen $500^{\circ} \mathrm{C}$ und $650^{\circ} \mathrm{C}$ geglüht wird.

Der Probenhalter (Abb. 4.5) besteht aus einem Standard Edelstahlträger mit einem isolierenden Stenan $\bigoplus$ Aufsatz, in welchem der Einkristall in einer angepassten halbkreisförmigen Öffnung eingebettet ist. Der Stenanhalter wird mit einer $0.1 \mathrm{~mm}$ dicken

\footnotetext{
${ }^{9}$ Stenan wird als Alternative zum üblicherweise benutzten Macor verwendet, da auch bei hohen Temperaturen keine ausgasenden Materialien gefunden werden. Bei Macor gasen mit zunehmender Temperatur Wasserstofffluorid-Komponenten (HF) aus.
} 
Goldfolie überzogen, welche den Einkristall fixiert und elektrisch kontaktiert. Der flächendeckende Überzug der Goldfolien verhindert außerdem, dass während des Sputterns ein anderes Material als Gold abgetragen wird, welches ansonsten die Einkristall- Oberfläche kontaminieren würde. Nicht zuletzt hilft der Goldfolienüberzug auch, den Stenanhalter gleichmäßig zu erhitzen und damit ein Zerbersten aufgrund thermisch induzierter mechanischer Spannungen zu verhindern.

\section{a) Vorpräparation}

Zum Beseitigen von Oberflächenkontaminationen und gleichzeitigem Einebnen des Kristalls werden aufeinanderfolgende Sputter- und Heizzyklen verwendet. Der Sputterprozess dient zum Abtragen der Oberfläche durch hochenergetischen Beschuss mit Argon-Ionen ${ }^{[0]}$ Der anschließende Glühprozess wird zum Einebnen der Oberfläche durchgeführt. Hierzu wird der in Kap. 4.1.2 beschriebene Ofen benutzt.

Der wesentliche Teil der Vorpräparation wird vom Sputterprozess getragen, da in erster Linie möglichst viele Atomablagen abgetragen werden sollen. Typische Sputterzeiten liegen im Bereich zwischen 1 und 5 Stunden, denen ein Heizprozess von etwa 20 Minuten bei ca. $650^{\circ} \mathrm{C}$ folgt. Stichprobenartig wird nach einigen Präparationszyklen die Oberfläche mit einem Rasterkraftmikroskop [DI] untersucht.

Die Abbildungen 4.9-4.12 verdeutlichen den Fortgang der Vorpräparation: Der noch unpräparierte polierte Kristall (Abb. 4.9) ist vor allem von etwa $30 \mathrm{~nm}$ tiefen Schleifriefen geprägt. Nach etwa 6 Stunden Sputtern (Abb. 4.10) sind diese Furchen zwar noch immer gut erkennbar, die Tiefe dieser Rillen beträgt jedoch nur noch ca. 10-20 nm. Nach 24 Stunden Sputtern (Abb. 4.11) sind erstmals deutliche Hinweise für eine fcc-(111)-Oberfläche zu erkennen; neben monatomaren Stufen und Terrassen der Höhe $0.24 \mathrm{~nm}$ (entsprechend dem Erwartungswert für Gold) sind auch einige geradlinige Stufen erkennbar, die einen $60^{\circ}$-Winkel zueinander einschließen. Sie stammen von Versetzungen, die entlang den (111) Ebenen aus dem Kristallinnern herausgewandert sind und beim Durchkreuzen der Oberfläche eine monatomare Stufe in eine der drei zu $[1 \overline{1} 0]$ äquivalenten Richtungen erzeugen. Die monatomaren Stufen sind jedoch noch an einigen punktförmigen Verunreinigungen von etwa $10 \mathrm{~nm}$ Höhe geheftet. Nach 36 Stunden schließlich (Abb. 4.12) Sind auch diese Verunreinigungen weitestgehend verschwunden und es bleiben atomar glatte Terrassen mit Weiten bis zu etwa $1 \mu \mathrm{m}$ übrig.

\footnotetext{
${ }^{10}$ Es wird die in Kap. 4.1.2 beschriebene Ionen Quelle verwendet. Während der Vorpräparation wird ein Ionenstrom von $15 \mu \mathrm{A}$ bei einem Argon- Partialdruck von etwa $7 \cdot 10^{-6}$ mbar erzeugt. Die Ionenenergie beträgt $700 \mathrm{eV}$.
} 


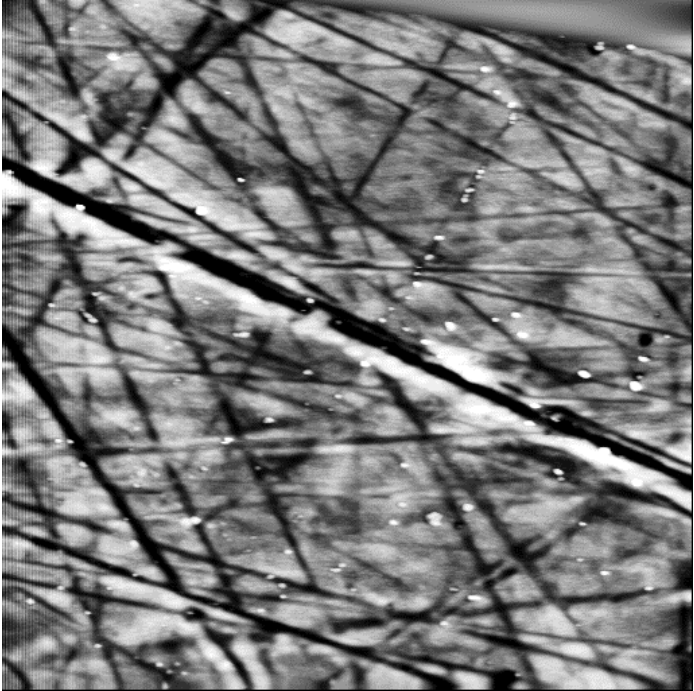

Abb. 4.9 Topografie des Au(111) Einkristalls vor der Präparation $\left(8 \times 8 \mu \mathrm{m}^{2}\right)$

$0 \mathrm{~nm} \square 20 \mathrm{~nm}$

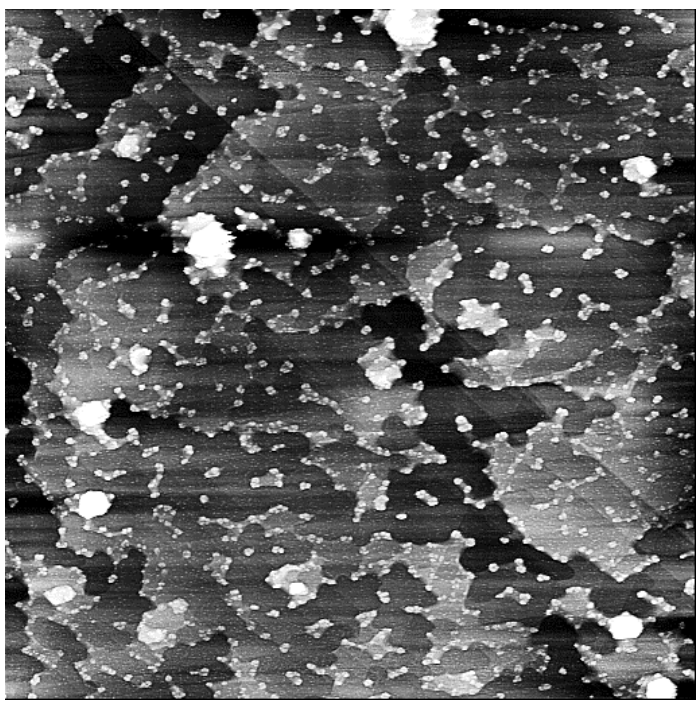

Abb. 4.11 ... nach 24 Stunden Sputtern $\left(8 \times 8 \mu \mathrm{m}^{2}\right)$ $0 \mathrm{~nm} \square 20 \mathrm{~nm}$

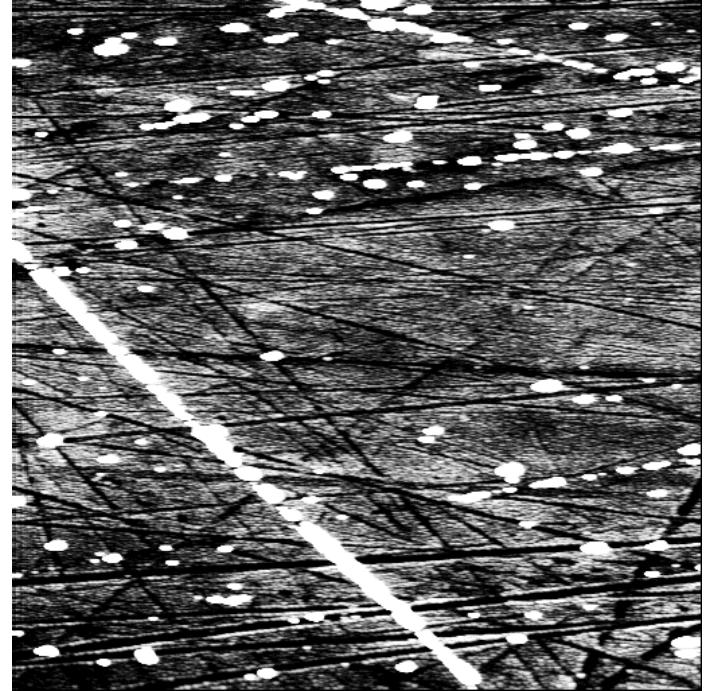

Abb. 4.10 ... nach 6 Stunden Sputtern $\left(8 \times 8 \mu \mathrm{m}^{2}\right)$

$0 \mathrm{~nm} \amalg 20 \mathrm{~nm}$

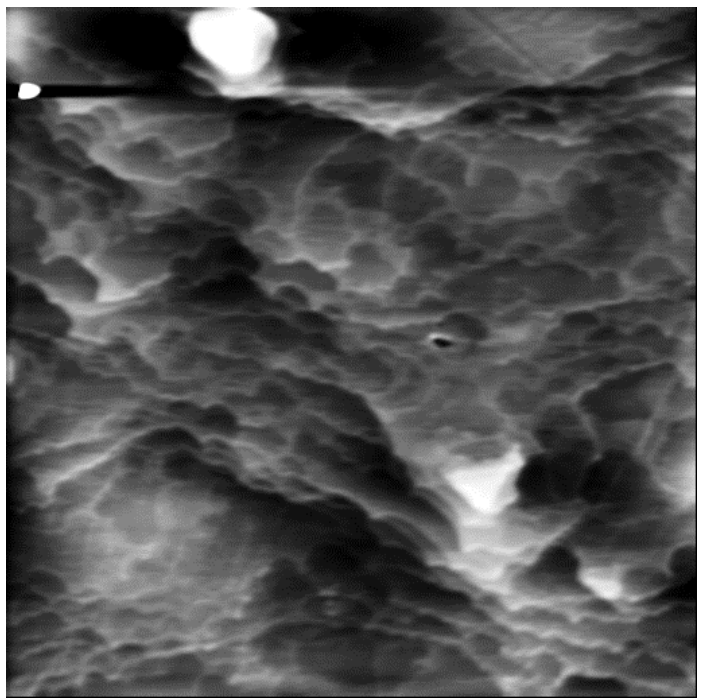

Abb. 4.12 ... nach 36 Stunden Sputtern $\left(8 \times 8 \mu \mathrm{m}^{2}\right) \quad 0 \mathrm{~nm} \square 10 \mathrm{~nm}$

Im nächsten Schritt der Vorpräparation wird darauf hingearbeitet, die Konzentration von Fremdatomen an der Oberfläche so weit wie möglich zu reduzieren. Dies geschieht durch alternierend ausgeführte relativ kurze Sputter- und Glühzyklen. Die Glühtemperatur und Glühdauer wird hierbei mit jedem Heizschritt ein wenig verringert. Es wird angenommen, dass sich Fremdatome bevorzugt auf der Oberfläche ansammeln und dabei in der Nähe der Oberfläche eine an Fremdatomen verarmte Zone hinterlassen [Bür 1]. Die oberste Atomlage wird dann nach jedem Heizvorgang durch kurzes Sputtern entfernt. 
Die letzten drei Zyklen der Vorpräparation werden deshalb mit Sputterzeiten von jeweils $2 \mathrm{~h}$, $1 \mathrm{~h}$ und zuletzt 30 Minuten abgeschlossen, denen jeweils 15 minütige Heizprozesse bei $560^{\circ} \mathrm{C}, 545^{\circ} \mathrm{C}$ und zuletzt $530^{\circ} \mathrm{C}$ folgen.

\section{b) Standardpräparation vor der Untersuchung im STM}

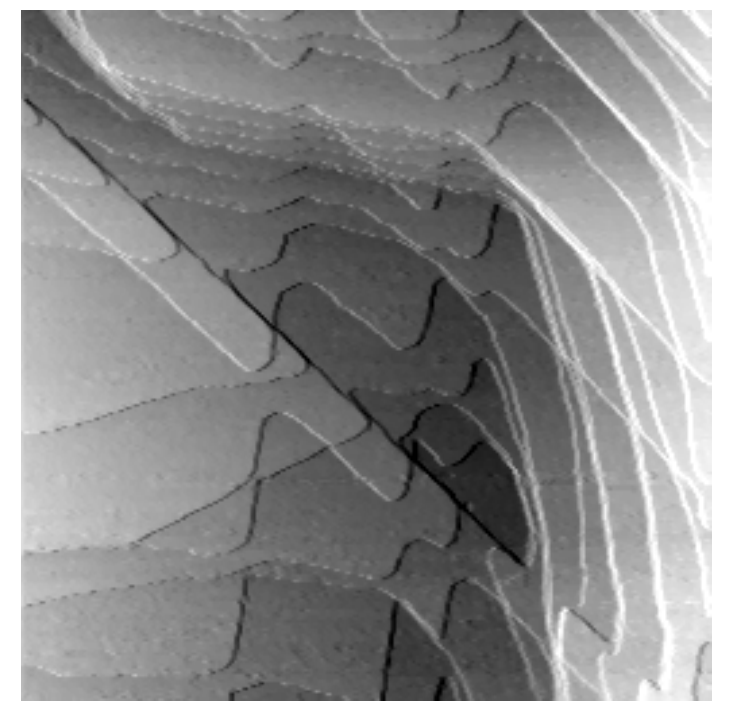

Abb. 4.13 Topografie einer Einkristalloberfläche nach erfolgter Präparation $\left(2 \times 2 \mu \mathrm{m}^{2}\right)$

$0 \mathrm{~nm} \longleftarrow 2.5 \mathrm{~nm}$

Nach abgeschlossener Vorpräparation ist nur noch ein relativ geringer Aufwand für die Schaffung einer reinen Oberfläche nötig. Wenn der Kristall zuvor an Luft lagerte, werden drei aufeinanderfolgende Sputterprozesse von jeweils 15 Minuten durchgeführt, denen jeweils 15 minütige Glühprozesse bei $530^{\circ} \mathrm{C}$ folgen. Bei längerer Lagerung im UHV wird nur ein Sputter- und Glühprozess vorgenommen. Im Gegensatz zur Vorpräparation spielt hier der Glühprozess die entscheidende Rolle. Durch das Glühen wird die Oberflächendiffusion exponentiell mit der Temperatur beschleunigt, was innerhalb der Glühdauer zu folgenden Effekten führt:

- Kleinere Inseln (bis zu mehreren $100 \mathrm{~nm}$ Durchmesser), welche sich während des Sputterprozesses bei Raumtemperatur bilden, werden durch Ostwaldreifung aufgelöst [Mor 3. Haa]. Die von den Inseln abgelösten Atome lagern sich bevorzugt an langgestreckten Stufen an.

- Sputterinduzierte Punktdefekte (Leerstellen und Argon-Ionen) werden ausgeheilt Haa]

Als Schattenseite einer erhöhten Diffusionsgeschwindigkeit wird leider auch die Konzentration von Fremdatomen an der Oberfläche stetig vergrößert. Die 15 Minuten Glühzeit stellen somit einen empirisch gefundenen Kompromiss zwischen obigen erwünschten topografischen Effekten und unerwünschter Fremdatomdiffusion dar. Nach Ende der Präparation erhält man eine Oberfläche, wie sie in Abb. 4.13 dargestellt ist. 


\title{
4.4.3. Spitzenpräparation
}

Für die Messungen werden Wolframspitzen verwendet, welche elektrochemisch im Lamellenätzverfahren mit $2 \mathrm{~m} \mathrm{NaOH}$ - Lösung bei einer angelegten Wechselspannung von $0.5 \mathrm{~V}-2 \mathrm{~V}$ und einer Stromstärke von $2-10 \mathrm{~mA}$ hergestellt werden. Beim Lamellenätzverfahren wird der Wolframdraht von einer Drahtschlaufe von ca. $3 \mathrm{~mm}$ Durchmesser umgeben, innerhalb derer sich eine Lamelle aus $\mathrm{NaOH}$ - Lösung befindet. Zwischen Drahtschlaufe und Wolframdraht wird die Wechselspannung angelegt. Nach dem Durchätzen des Wolframdrahtes wird das untere Drahtstück aufgefangen und in einem Spitzenhalter in das UHV eingeschleust.

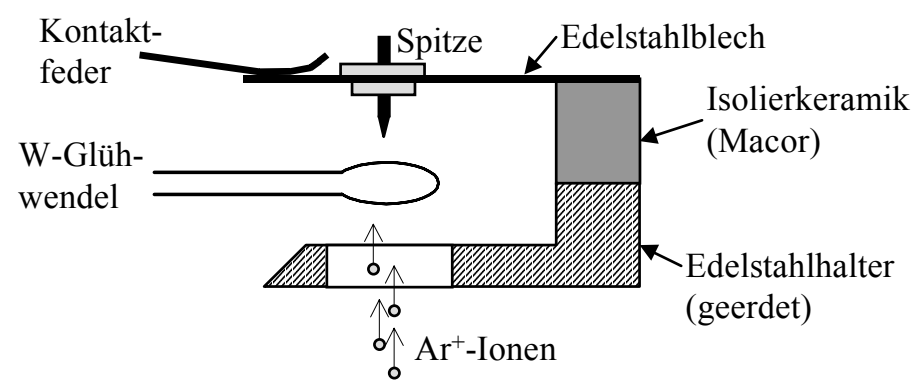

\author{
Abb. 4.14 Schematischer Aufbau \\ zur UHV-Präparation von Spitzen
}

In einer Spezialhalterung (Abb. 4.14) können Spitzen im UHV mit verschiedenen kombinierbaren Techniken präpariert werden. Die Spitze wird auf einem Edelstahlblech gehaltert, welches über eine Kontaktfeder auf ein definiertes Spannungspotenzial gelegt werden kann.

Im ersten Schritt werden die Spitzen eine halbe Stunde lang bei Rotglut (abgeschätzt zu etwa $850^{\circ} \mathrm{C}$ ) geglüht, wobei die Oxidschicht entfernt wird [Che 3]. Das Glühen findet unter Elektronenbeschuss statt, wobei die Spitze auf positives Potenzial (200-300 V bei $4.5 \mathrm{~mA}$ Stromfluss) gelegt wird. Als Kathode wird eine kreisförmig gebogene Wolfram-Glühwendel einer Halogenlampe verwendet.

Anschließend wird die Spitze mit Argon-Ionen mit einer Energie von $4 \mathrm{keV}$ zwischen 30 Minuten und $2 \mathrm{~h}$ gesputtert, um Adsorbate zu entfernen und den äußeren Spitzenradius zu verringern. Während des Sputterns wird die Spitze nach dem von D. Albrektsen und Mitarbeitern angegebenen Verfahren $[\mathrm{Alb}]$ im Feldemissionsmodus betrieben. Hierzu wird die Spitze über eine Regelelektronik auf ein negatives Potenzial $(0>\mathrm{U}>-1000 \mathrm{~V})$ gelegt, welches auf einen konstanten Feldemissionsstrom von $10 \mu \mathrm{A}$ gesteuert wird. Während des Sputterns nimmt die mittlere Feldemissionsspannung kontinuierlich ab, was nach D. Albrektsen auf eine Verringerung des äußersten Spitzenradius hinweist [Alb]. Das Sputtern der Spitze wird abgebrochen, sobald sich die Feldemissionswerte nicht mehr wesentlich verbessern.

Die Lagerung der Spitzen nach der Präparation bis zum Gebrauch geschieht unter UHVBedingungen $\left(<1 \cdot 10^{-9}\right.$ mbar $)$. 


\section{Oberflächenzustände}

Bei der theoretischen Beschreibung der elektronischen Struktur von Festkörpern geht man im allgemeinen von der Annahme aus, dass sich der Festkörper unendlich weit ausdehnt. Hierauf aufbauend erhält man die wohlbekannten Modelle elektronischer Bandstrukturen [Ash] Allen Modellen mit periodischen Potenzialen ist dabei gemeinsam, dass die Wellenfunktion eines jeden Elektrons über den gesamten Kristall hinweg ausgedehnt ist, weshalb diese Zustände Volumenzustände genannt werden (Abb. 5.1 a)

Die Situation ändert sich grundlegend, wenn eine Oberfläche geschaffen wird und damit die Kristallsymmetrie gebrochen wird. Die zuvor periodischen sog. Bloch-Wellenfunktionen der Volumenzustände müssen zur Vakuumseite hin exponentiell abklingen $\llbracket$ Es können jetzt jedoch auch innerhalb der Bandlücken stationäre Lösungen für die Schrödinger-Gleichung gefunden werden, die an der Oberfläche lokalisiert sind und in den Kristall hinein exponentiell abklingen; diese werden Oberflächenzustände genannt (Abb. 5.1 b)

Es kann auch Wellenfunktion geben, welche ein erhöhtes Betragsquadrat an der Oberfläche besitzen, sich aber auch in den Kristall hinein ausdehnen. Diese werden mit Oberflächenresonanzen bezeichnet ${ }^{12}$ (Abb. $\left.5.1 \mathrm{c}\right)$.

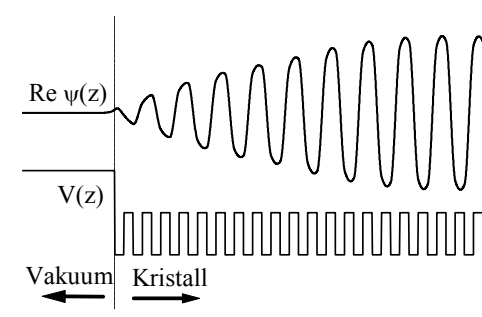

(a) Volumenzustand

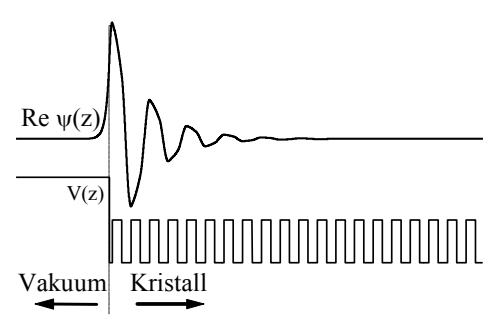

(b) Oberflächenzustand

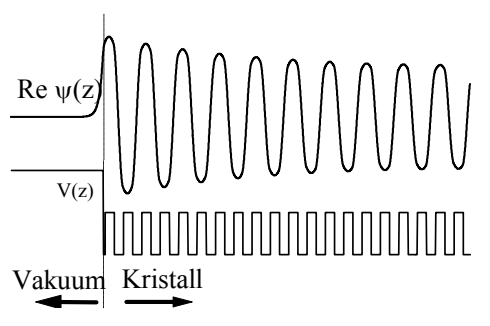

(c) Oberflächenresonanz

Abb. 5.1 Drei mögliche Wellenfunktionen (oberer Graph: Realteil) für einen eindimensionalen, halbseitigen Kristall mit Kronig-Penney-Potenzial ([Kro], unterer Graph).

Aufgrund der Anschlussbedingung an die Vakuumwellenfunktion bestimmt die Form des Oberflächenpotenzials sehr empfindlich darüber, ob Oberflächenzustände überhaupt existieren können, bzw. bei welcher diskreten Energie innerhalb der Bandlücke sie gefunden werden.

Die Existenz von Oberflächenzuständen wurde erstmals 1932 von I. Tamm anhand eines halbseitigen, eindimensionalen Kronig-Penney Potenzialmodells in z-Richtung senkrecht zur Oberfläche vorhergesagt [Tam 1-2], Er berechnete die Lösungen der Schrödingergleichung für die Kristallhälfte und die Vakuumseite getrennt voneinander und schloss diese dann an der Oberfläche stetig differenzierbar aneinander an (Abb. 5.2)

\footnotetext{
${ }^{11}$ Im folgenden sollen nur Elektronen betrachtet werden, die nicht aus dem Kristall herauspropagieren können, d.h. in Eigenzuständen mit Energien unterhalb der des Vakuumniveaus.

${ }^{12}$ Eine grundlegende theoretische Behandlung von Oberflächenzuständen ist z.B. in [Dav 1] gegeben.
} 
1D-Potential: $\quad$ konstant für $z<0$, Kronig-Penney-Potential für $z>0 \quad$ (siehe Abb. 5.1)

$\begin{array}{lll}\text { Lösung für Vakuumseite: } & \psi \propto \mathrm{e}^{\mathrm{bz}} & ; \mathrm{b}>0 \\ \text { Lösung für Kristallhälfte: } & \psi \propto \mathrm{u}_{\mathrm{k}}(\mathrm{z}) \cdot \mathrm{e}^{\mathrm{ikz}} & ; \mathrm{u}_{\mathrm{k}}(\mathrm{z}) \text { ist gitterperiodisch }\end{array}$

für reelle k: $\rightarrow$ Volumenzustände

für komplexe k: $\rightarrow$ Oberflächenzustand

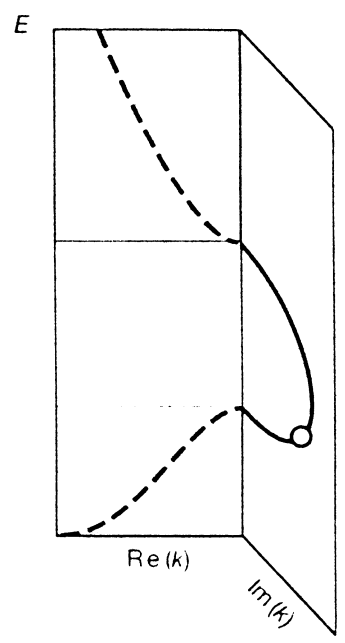

Notwendige Randbedingungen für den Oberflächenzustand:

- Imaginärteil von $\mathrm{k}$ lässt Wellenfunktion in der Kristallhälfte abklingen

- Realteil von k liegt am Rand der Brillouin-Zone

- Anschluss von Vakuum- und Kristallwellenfunktion möglich

Gestrichelte Linie: Lösung für unendlich ausgedehntes Kristall-Potential.

Durchgezogene Linie: Zusätzliche Lösungen des KristallHalbraumes ohne Anschluss der Vakuumwellenfunktion.

Kreis: Die einzige Lösung für einen Oberflächenzustand mit Anschluss der Vakuumwellenfunktion.

Abb. 5.2 Oberflächenzustände in der Behandlung nach I. Tamm [Tam 1-2]; Zeichnung nach N. Memmel Mem].

W. Shockley verwendete 1939 einen alternativen Zugang, in dem er zeigte, wie Oberflächenzustände aus den diskreten Zuständen von Atomen entstehen, wenn sie aus dem Unendlichen heraus auf endliche Abstände angenähert werden [Sho] Hierbei entstehen die Oberflächenzustände, sobald sich die äußeren Atomorbitale bei genügend kleinen Atomabständen hinreichend überlappen. Aus den atomaren Levels entstehenden Bänder. Wie bei Tamm sind die Oberflächenzustände innerhalb der Bandlücke für Volumenzustände anzufinden (Abb. 5.3),

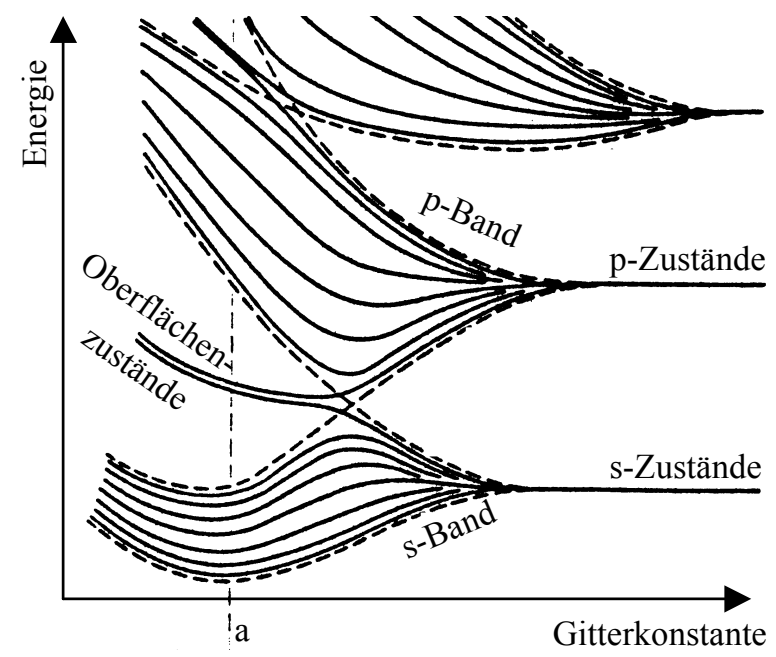

Abb. $\quad 5.3$ Energiebänder für ein eindimensionales Gitter mit acht Atomen, aufgetragen gegen die Gitterkonstante. Für hinreichend kleine Gitterkonstanten a entsteht ein Oberflächenzustand in der Bandlücke der sich überlappenden s- und p-Bänder (aus Ref. W. Shockley [Sho]]. 
Shockley analysierte ebenfalls die Erweiterung auf drei Dimensionen, wobei er die Existenz eines Bandes von Oberflächenzuständen vorhersagte. Demnach sind die Oberflächenzustände nur in eine Richtung senkrecht zur Oberfläche lokalisiert; parallel zur Oberfläche jedoch verhalten sie sich wie Blochwellen.

In der Folgezeit wurde in der Literatur zwischen Tamm- und Shockley-artigen Oberflächenzuständen unterschieden [Dav 1]. So werden Tamm-Zustände häufig mit „lokalisierten“ Oberflächenzuständen (sogenannten „Dangling Bonds“) in Verbindung gebracht, von denen man annimmt, dass sie räumlich hinreichend weit voneinander getrennt sind, so dass die Orbitalüberlappung vernachlässigt werden kann. Die Shockley-Zustände wiederum werden mit einem zweidimensionalen Elektronengas in Verbindung gebracht, welches bei einer Orbitalüberlappung für hinreichend kurze Atomabstände entsteht. Es ist jedoch zu beachten, dass Tamm und Shockley dieselbe Art von Oberflächenzuständen beschrieben haben, und die eben genannte Klassifizierung vielmehr historisch aus den unterschiedlichen methodischen Herangehensweisen entstanden ist.

Experimentell wurden Oberflächenzustände auf Halbleiteroberflächen erstmals 1947 [Mey, Bar 3, Bra] und auf Metalloberflächen erstmals 1967 [Swa] nachgewiesen. Kurze Zeit später lieferten Experimente mit winkelaufgelöster Photoemissionsspektroskopie (ㅁngelresolved Photoemission spectroscopy, „ARPES“) sehr genaue Einblicke in die elektronische Struktur der Oberfläche [Feu], ARPES ermöglichte, die Energie von Zuständen aufgelöst nach der Parallelkomponente $\mathrm{k}_{\|}$des Wellenvektors parallel zur Oberfläche zu bestimmen. 


\section{Die Au(111)-Oberfläche}

\subsection{Elektronische Struktur}

Die elektronische Struktur von $\mathrm{Au}(111)$ ist der von $\mathrm{Cu}(111)$ und $\mathrm{Ag}(111)$ sehr ähnlich. Alle diese Edelmetalle besitzen in ihrer atomaren Elektronenkonfiguration komplett gefüllte d-Niveaus, ein einfach besetztes s-Niveau und bilden fcc-Kristalle. In kristalliner Form überlappen sich, wie in Abb. 5.3 qualitativ dargestellt, das halb gefüllte s-Band mit dem unbesetzten p-Band. In die (111)-Richtung (der $\Gamma$ L-Richtung in der Bandstruktur von Abb. 6.4 bildet sich infolgedessen eine Bandlücke aus, welche von $\mathrm{E}_{\mathrm{F}}-0.9 \mathrm{eV}[\mathrm{Kev}]$ bis $\mathrm{E}_{\mathrm{F}}+3.6 \mathrm{eV}$ [Woo] reicht. Dies wird bei Betrachtung der Fermifläche von Gold Abb. 6.1 und 6.2 besonders deutlich. Diese besitzt eine nahezu sphärische Form mit sogenannten „Halsbahnen“ in allen acht gleichberechtigten (111)-Richtungen, welche die hexagonalen Flächen der Brillouinzone kreisförmig schneiden.

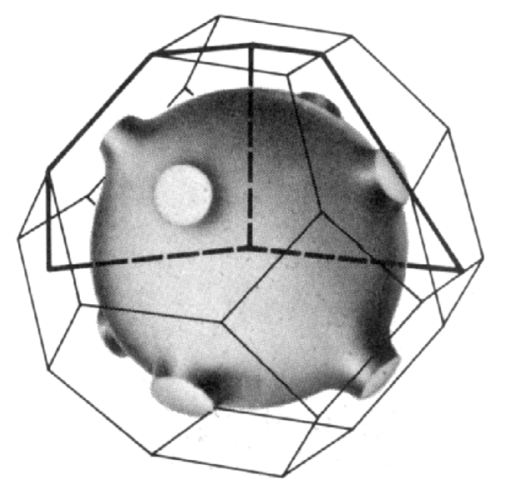

Abb. 6.1 Fermifläche von Gold (aus Ref. D. J. Roaf [Roa])

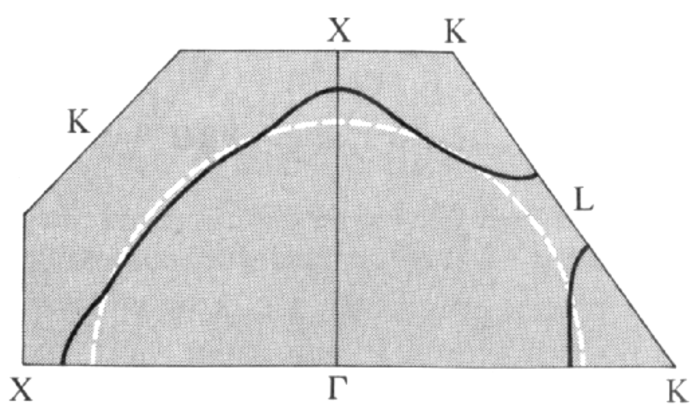

Abb. 6.2 Querschnitt durch die Fermifläche (aus Ref. D. J. Roaf [Roa])
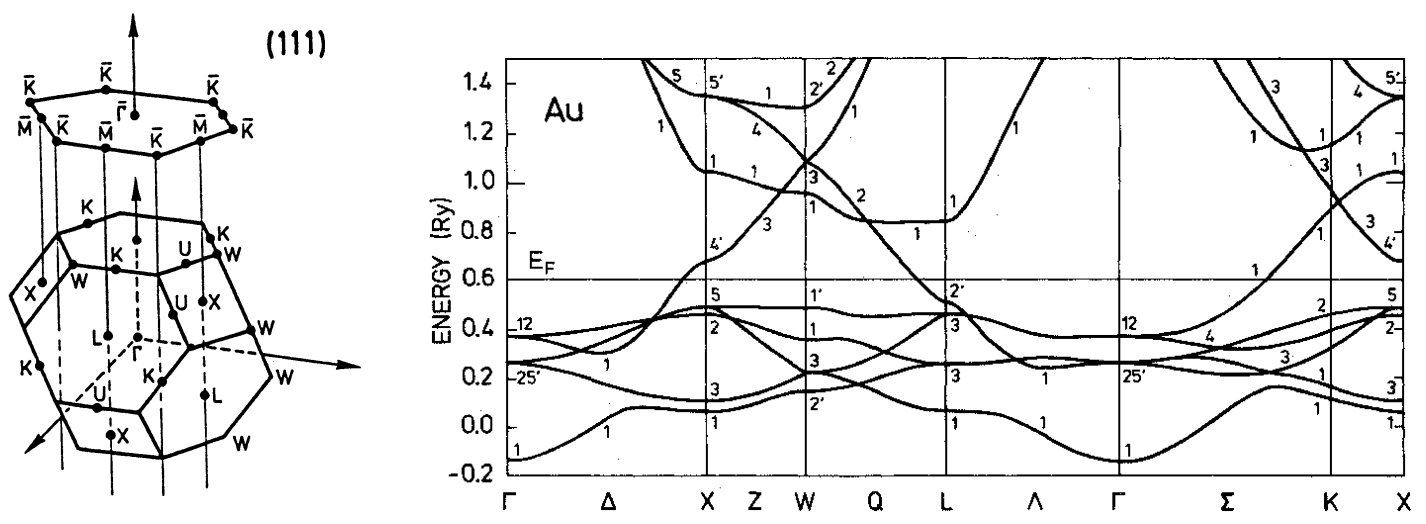

Abb. 6.3 Volumen- und Oberflächen- Brillouinzone Abb. 6.4 Volumenbandstruktur von Gold, berechnet mit Pseudoder Au(111)-Oberfläche (aus Ref. H. Lüth [Lüt]) potenzialmethode mit relativistischer Band-Shift Korrektur (aus Ref. O. Jepsen [Jep] 
Bei der Fermienergie gibt es somit keine in (111)-Richtung propagierenden Elektronenwellen.

Die Voraussetzungen für Oberflächenzuständen sind damit erfüllt, und der s-p-artige Oberflächenzustand wird tatsächlich bei allen drei Edelmetallen gefunden (Abb. 6.5).

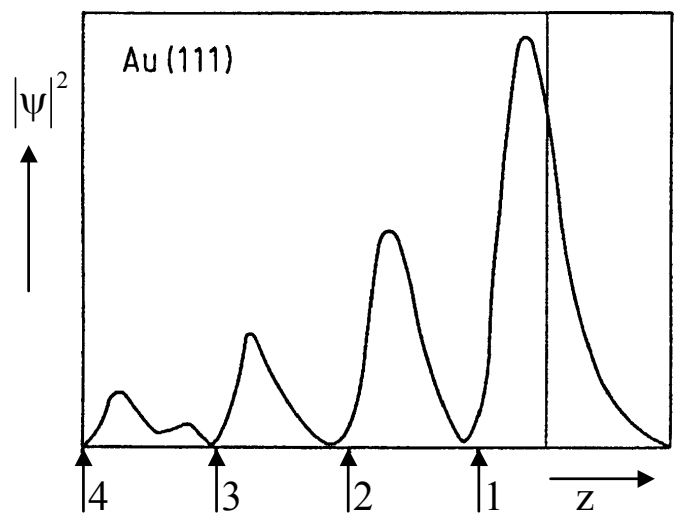
Abb. 6.5 Betragsquadrat des Oberflächenzustan- des von $\mathrm{Au}(111)$ (reproduziert aus Ref. S. H. Liu [Liu]]. Die untere Achse gibt die z-Koordinate senkrecht zur Oberfläche an. Die Zahlen 1-4 geben die Positionen der (111)-Netzebenen durch die Kernpositionen von der obersten Lage aus gezählt an.

Er wurde intensiv mittels winkelaufgelöster Photoemission [Gar, Gol 1, Kev, LaS, Mat 2, $\mathrm{McD}$, Pan, Qu, Rei 1, und k-aufgelöster inverser Photoemissions-Spektroskopie (,KRIPES“) [Hul 1-2, Gol 2, Woo untersucht. Die Ergebnisse dieser Experimente zeigen ein zweidimensionales Gas aus nahezu freien Elektronen mit parabolischer und isotroper Dispersionsrelation. Kürzlich jedoch stellten ARPES Experimente mit deutlich verbesserter Energie- und k-Auflösung erstmals eine qualitative Abweichung der Dispersionsrelation von $\mathrm{Au}(111)$ gegenüber denen von $\mathrm{Ag}(111)$ und $\mathrm{Cu}(111)$ fest. LaShell [LaS], Reinert [Rei 1] und Mitarbeiter wiesen nach, dass die Dispersionsrelation von $\mathrm{Au}(111)$ nicht aus einer, sondern aus zwei im k-Raum parallelverschobenen Parabeln besteht (Abb. 6.6) Alternativ zu ARPES kann die Dispersionsrelation von Au(111) auch mit dem STM ermittelt werden (Abb. 6.7). Bei dieser Technik wird ausgenutzt, dass Elektronenwellen aus Oberflächenzuständen an streuenden Oberflächendefekten, wie z.B. monatomaren Stufen, miteinander interferieren und ein Interferenzmuster bilden. Wenn das STM im Modus der dI/dU-Spektroskopie (STS) betrieben wird, können Interferenzmuster selektiv nach der Elektronenenergie aufgelöst kartiert werden. Durch vermessen der Wellenlängen im Ortsraum kann dann direkt die Dispersionsrelation bestimmt werden. 


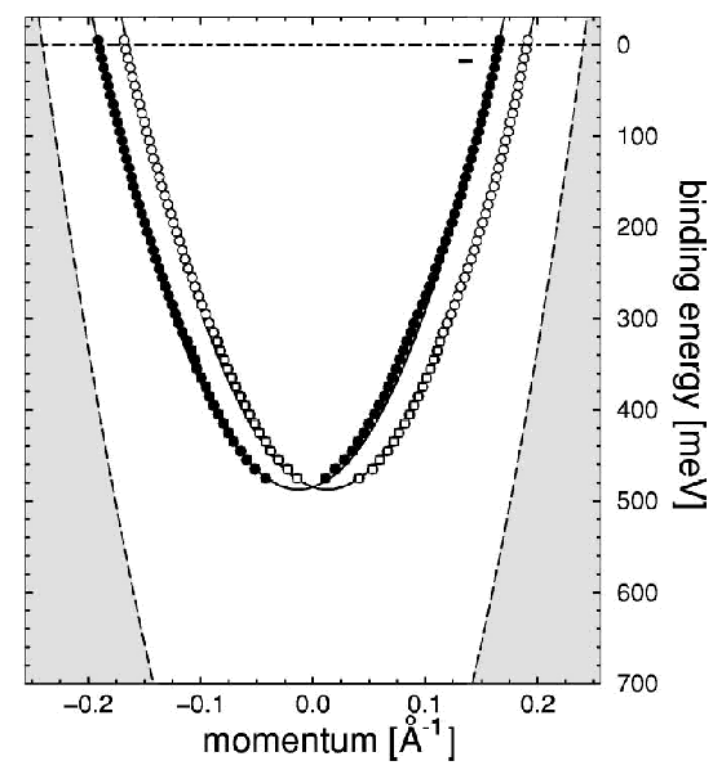

Abb. 6.6 Dispersionsrelation des Oberflächenzustandes von $\mathrm{Au}(111)$, bestimmt mittels ARPES. Aufgrund von Spin-Bahn Aufspaltung entstehen zwei entlang von $\mathrm{k}_{\|}$parallelverschobene Parabeln (aus Ref. F. Reinert [Rei 1]).

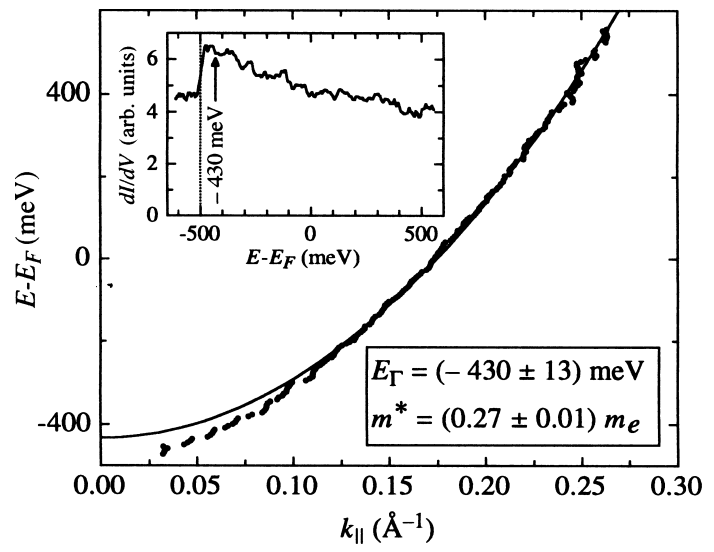

Abb. 6.7 Dieselbe Dispersionsrelation, bestimmt mit dem STM (aus Ref. L. Bürgi [Bür 1]). Die Spin-Bahn-Aufspaltung kann mit diesem Verfahren prinzipiell nicht beobachtet werden [Pet 6]

Im Kontrast zu ARPES wird mit dem STM jedoch mit hoher Messgenauigkeit nur eine einzelne Parabel als Dispersionsrelation gefunden. Diese sich scheinbar widersprechenden Ergebnisse sind jedoch miteinander vereinbar, wenn eine Spin-Bahn-Aufspaltung der Oberflächenzustände angenommen wird ${ }^{[3]}$ [Pet 6]. Aufgrund des Umfanges wird diese Thematik gesondert im nächsten Abschnitt behandelt. An dieser Stelle sei nur erwähnt, dass die mit dem STM gemessenen Interferenzeffekte mit einer Dispersionsrelation $\varepsilon\left(\mathrm{k}_{\|}\right)$ interpretiert werden können, welche aus nur einer einzelnen Parabel besteht:

$$
\varepsilon\left(\mathrm{k}_{\|}\right)=\frac{\hbar^{2} \mathrm{k}_{\|}^{2}}{2 \mathrm{~m}^{*}}-\left(\mu_{\mathrm{ec}}-\mathrm{E}_{\Gamma}\right)
$$

$\mathrm{E}_{\Gamma}$ steht hier für die Energie der unteren Bandkante der Oberflächenzustände. Die Dispersionsrelation für $\mathrm{Au}(111)$ ist jedoch nicht perfekt parabolisch. Wie experimentelle Ergebnisse von L. Bürgi [Bür 1] in Abb. 6.7 zeigen, gibt es für Energien kleiner als ca. $300 \mathrm{meV}$ unterhalb der Fermienergie Abweichungen von der Parabelform. Ursache hierfür ist das effektive Potenzial der Oberflächenrekonstruktion, welches eine Modulationstiefe von etwa $40 \mathrm{meV}$ besitzt und somit für Elektronen niedriger Energie die Modellannahme freier Elektronen nicht mehr erfüllt ist [Che 1: Bür 1]. Für die in den nächsten Abschnitten vorgestellten STM-Messungen spielt diese Einschränkung keine nennenswerte Rolle, da hier

\footnotetext{
${ }^{13}$ Anfängliche Vermutungen, wonach die Aufspaltung mit der Oberflächenrekonstruktion von Au(111) zusammenhängt, konnten glaubhaft ausgeräumt werden [LaS].
} 
hauptsächlich Elektronen im Bereich der thermischen Verbreiterung nahe der Fermienergie betrachtet werden.

Zusammenfassend ist in Tab. 6.1 eine Auswahl experimenteller Ergebnisse zur Dispersionsrelation von $\mathrm{Au}(111)$ wiedergegeben.

\begin{tabular}{|c|c|c|c|c|c|c|}
\hline $\begin{array}{l}\mathrm{E}_{\Gamma}-\mu_{\mathrm{ec}} \\
{[\mathrm{meV}]}\end{array}$ & $\mathrm{m}^{*} / \mathrm{m}_{\mathrm{e}}$ & $\begin{array}{l}\mathrm{k}_{\mathrm{F}} \\
{\left[\mathrm{nm}^{-1}\right]}\end{array}$ & $\mathrm{T}[\mathrm{K}]$ & $\begin{array}{l}\text { Energiefenster } \\
\left(\text { rel. } \mathrm{zu} \mu_{\mathrm{ec}}\right)\end{array}$ & Methode & Referenz \\
\hline \multirow[t]{2}{*}{$-408 \pm 10$} & $\begin{array}{l}0.284 \\
\pm 0.003\end{array}$ & $\begin{array}{l}1.74 \\
\pm 0.05\end{array}$ & 300 & $\mathrm{E}_{\Gamma}$ bis $\mu_{\mathrm{ec}}$ & ARPES & {$[\mathrm{Kev}]$} \\
\hline & $(0.15)^{\mathrm{a})}$ & & 300 & $\begin{array}{l}-275 \mathrm{meV} \text { bis } \\
+450 \mathrm{meV}\end{array}$ & STM & $\begin{array}{l}\text { [Has 1]. } \\
\text { [Hör 1-2] }\end{array}$ \\
\hline$-468 \pm 20$ & & & $50-150$ & Nur bei $E_{\Gamma}$ & ARPES & [Pan] \\
\hline$-440 \pm 20$ & & & 300 & Nur bei $E_{\Gamma}$ & ARPES & [Pan] \\
\hline$-417 \pm 1$ & 0.25 & $\begin{array}{l}1.53 ; \\
1.76 \text { घ) }\end{array}$ & 300 & $\mathrm{E}_{\Gamma}$ bis $\mu_{\mathrm{ec}}$ & ARPES & [LaS] \\
\hline-520 & 0.26 & & 4 & & STM & [Che 1] \\
\hline $\begin{array}{l}-430 \pm 13 \\
(-510)\end{array}$ & $\begin{array}{l}0.27 \\
\pm 0.01\end{array}$ & 1.7 & 5 & $\begin{array}{l}-300 \mathrm{meV} \text { bis } \\
+600 \mathrm{meV}\end{array}$ & STM & [Bür 1] \\
\hline$-487 \pm 1$ & 0.255 & $\begin{array}{l}1.67 ; \\
1.92 \text { b) }\end{array}$ & 30 & $\mathrm{E}_{\Gamma}$ bis $\mu_{\mathrm{ec}}$ & ARPES & [Rei 1] \\
\hline
\end{tabular}

Tab. 6.1 Experimentell bestimmte Daten zur Dispersionsrelation von Au(111) für die Bandkantenenergie $\mathrm{E}_{\Gamma}$, effektive Masse $\mathrm{m}^{*}$ und Fermi-Wellenzahl $\mathrm{k}_{\mathrm{F}}$.

a) Die relativ geringe effektive Masse konnte in einer späteren theoretischen Arbeit Hör 1-2] erklärt werden. Die durchgeführte Korrektur ergibt eine Bestätigung der Daten von [Kev].

b) Es werden zwei spinaufgespaltene Parabeln gemessen, s. Abb. 6.6.

c) $\mathrm{E}_{\Gamma}=-430 \mathrm{meV}$ ist hier nur als Fit-Parameter für Energien oberhalb von $-300 \mathrm{meV}$ relativ zu $\mu_{\mathrm{ec}}$ angegeben. Unterhalb dieser Energie wurden Abweichungen von einer parabelförmigen Dispersion gemessen, mit einer unteren Bandkantenergie von $\mathrm{E}_{\Gamma}=-510 \mathrm{meV}$. 


\subsection{Spin-Bahn-Kopplung}

Dieser Abschnitt erläutert im Modell freier Elektronen, warum eine Spin-Bahn-Aufspaltung der Dispersionsrelation zwar mittels ARPES gefunden wird (Abb. 6.6), jedoch nicht mit dem STM bei der Analyse von Interferenzmustern beobachtet wird ${ }^{14}$ Abb. 6.7).

Betrachtet man die Volumenbandstruktur von Gold, dann sind die Bänder entartet bezüglich der Spin-Bahn-Kopplung. Der Grund hierfür liegt in der Inversionssymmetrie der kubischflächenzentrierten Kristallstruktur, wie im untenstehenden Kasten (Abb. 6.8) näher erläutert wird.

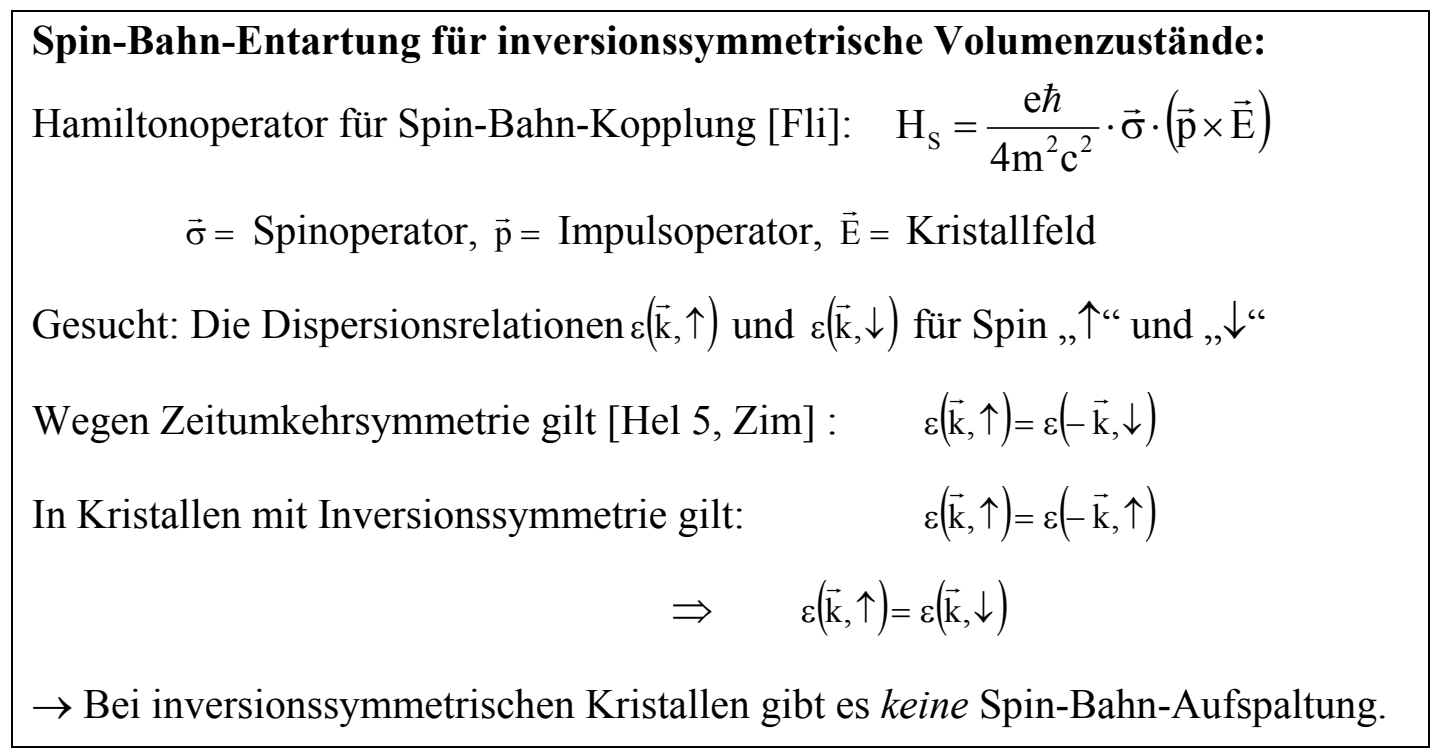

Abb. 6.8 (Nicht vorhandene) Spin-Bahn-Kopplung von Volumenzuständen

Die Inversionssymmetrie des Kristalls ist jedoch im Falle einer Oberfläche nicht mehr gegeben. S. LaShell und Mitarbeiter[LaS] konnten nachweisen, dass in diesem Fall die SpinBahn-Entartung aufgehoben wird (Abb. 6.9).

Die experimentell beobachtete Aufspaltung beträgt $110 \mathrm{meV}$ [LaS] bzw. $120 \mathrm{meV}$ Rei 1]. Diese Werte stimmen annehmbar mit dem theoretisch berechneten von $150 \mathrm{meV}$ überein [LaS],

14 Bei einer Überlagerung von Wellen mit unterschiedlichen Wellenvektoren müsste in den Interferenzmustern an monatomaren Stufen klar erkennbar eine Art „Schwebung“ existieren, welche jedoch experimentell noch nicht einmal ansatzweise gefunden wird.

${ }^{15}$ Mit ARPES wurde bisher noch keine Spinaufspaltung der Oberflächenzustände auf $\mathrm{Ag}(111)$ und $\mathrm{Cu}(111)$ beobachtet. Die relativ niedrige Spin-Bahn-Kopplungsstärke von $\mathrm{Ag}$ und $\mathrm{Cu}$ ist möglicherweise der Grund dafür. Andererseits sollte die Spinaufspaltung mit der aktuell möglichen Energieauflösung von ARPES bereits messbar sein [Rei 1], so dass die Frage der Spinaufspaltung der Oberflächenzustände auf $\operatorname{Ag}(111)$ und Cu(111) noch ungeklärt ist. 


\section{Spin-Bahn-Aufspaltung bei Oberflächenzuständen:}

Eine Oberfläche bricht die Inversionssymmetrie.

Modellannahme: Freies 2D-Elektronengas in einem Oberflächenpotenzial V(z),

$$
\Rightarrow \quad \overrightarrow{\mathrm{E}}=\frac{1}{\mathrm{e}} \cdot \frac{\partial \mathrm{V}}{\partial \mathrm{z}} \cdot \overrightarrow{\mathrm{e}}_{\mathrm{z}} \quad ; \overrightarrow{\mathrm{e}}_{\mathrm{z}} \text { ist Oberflächen-Normalenvektor. }
$$

Der Hamiltonoperator wird in einer Form $H=\frac{p^{2}}{2 m}+\alpha_{R} \cdot \vec{\sigma} \cdot\left(\vec{p} \times \vec{e}_{z}\right)$ genähert. $\alpha_{R}$ ist eine Konstante (die „Spin-Bahn-Kopplungsstärke“), welche den Gradienten $\frac{\partial \mathrm{V}}{\partial \mathrm{z}}$ des Oberflächenpotenzials berücksichtigt.

Die analytische Lösung von $\mathrm{H}$ ergibt eine Spinaufspaltung der Dispersionsrelation:

$$
\varepsilon\left(\overrightarrow{\mathrm{k}}_{\|}\right)=\frac{\hbar^{2} \mathrm{k}_{\|}^{2}}{2 \mathrm{~m}^{*}} \pm \alpha_{\mathrm{R}}\left|\overrightarrow{\mathrm{k}}_{\|}\right| .
$$

Richtung der Spins: $\pm \overrightarrow{\mathrm{k}}_{\|} \times \overrightarrow{\mathrm{e}}_{\mathrm{z}}$

$\rightarrow$ Spin ist senkrecht zu $\overrightarrow{\mathrm{k}}_{\|}$und liegt in der Oberflächenebene (s. Abb. 6.10).

Abb. 6.9 Spin-Bahn-Kopplung von Oberflächenzuständen nach S. LaShell [LaS]

Eine Erklärung, warum diese Aufspaltung nicht in vom STM aufgenommenen Interferenzmustern auftritt, wurde kürzlich von L. Petersen und P. Hedegård gegeben [Pet 6]. Eine Schlüsselrolle spielt demnach das „Kramersche Theorem“ [Hel 5, Pet 6]. Dieses besagt, dass in Systemen mit Spin $\pm 1 / 2$ die Anwendung des Zeitumkehroperators $T$ auf einen Hamilton-Eigenzustand $\psi(\overrightarrow{\mathrm{k}}, \uparrow)$ wieder einen Eigenzustand $\psi(-\overrightarrow{\mathrm{k}}, \downarrow)$ ergibt.

Dieser ist zum ursprünglichen Eigenzustand orthogonal (d.h. es gilt $\langle\psi|\mathrm{T}| \psi\rangle=0$ ), hat denselben Energieeigenwert und besitzt einen Wellenvektor und Spin mit umgekehrten Vorzeichen ${ }^{16}$ (Abb. 6.10 und 6.11).

Die Orthogonalität dieser Wellenfunktionen hat zur Folge, dass eine Elektronenwelle mit Wellenvektor $\overrightarrow{\mathrm{k}}$ nicht mit der zeitumgekehrten, ,rücklaufenden“ Welle mit Wellenvektor $-\overrightarrow{\mathrm{k}}$ interferieren kann.

Bei einer spinaufgespaltenen Dispersionsrelation gibt es jedoch zu einer Energie zwei mögliche Wellenvektorbeträge $\mathrm{k}_{1}$ und $\mathrm{k}_{2}$ (Abb. 6.11). und es ist sehr wohl eine Interferenz von Wellen mit Wellenvektor $-\overrightarrow{\mathrm{k}}_{1}$ und $\overrightarrow{\mathrm{k}}_{2}$ bzw. $\overrightarrow{\mathrm{k}}_{1}$ und $-\overrightarrow{\mathrm{k}}_{2}$ möglich. Dies wird im folgenden an einem Beispiel veranschaulicht.

\footnotetext{
${ }^{16}$ Dies entspricht einer rückwärts laufenden „Filmaufnahme“ eines propagierenden Elektrons mit Spin.
} 


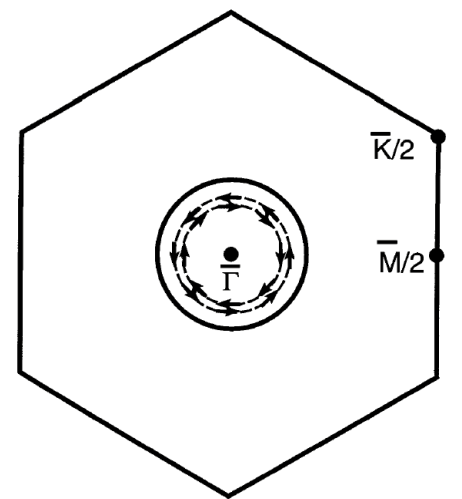

Abb. 6.10 Spin-Bahn-Aufspaltung in der ersten Brillouinzone von $\mathrm{Au}(111)$ bei $\mathrm{E}_{\mathrm{F}}$. Die beiden inneren Kreise stellen die aufgespaltenen Oberflächenzustände dar. Die Pfeile symbolisieren die in der Ebene liegende Spinrichtung. Der äußere Kreis stellt die „Halsbahn“ der entarteten Volumenzustände dar (aus Ref. S. LaShell [LaS]).

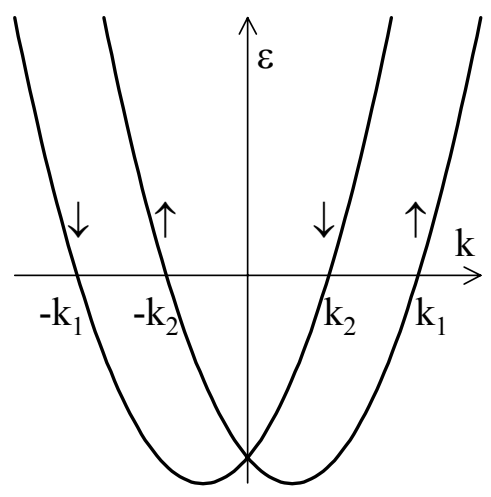

Abb. 6.11 Schematische Darstellung der spinaufgespaltenen Dispersionsrelation

Eine auf einen Streuer fallende Welle kann als Linearkombination der zu $\overrightarrow{\mathrm{k}}_{1}$ und $\overrightarrow{\mathrm{k}}_{2}$ gehörenden Wellenfunktionen geschrieben werden:

$$
\psi_{\text {in }}=\mathrm{A}_{1} \cdot\left|\overrightarrow{\mathrm{k}}_{1}, \uparrow\right\rangle+\mathrm{A}_{2} \cdot\left|\overrightarrow{\mathrm{k}}_{2}, \downarrow\right\rangle
$$

Die reflektierte Welle setzt sich aus der Linearkombination der beiden zueinander orthogonalen Spin-Wellenfunktionen zusammen:

$$
\psi_{\text {out }}=\mathrm{A}_{3} \cdot\left|-\overrightarrow{\mathrm{k}}_{1}, \downarrow\right\rangle+\mathrm{A}_{4} \cdot\left|-\overrightarrow{\mathrm{k}}_{2}, \uparrow\right\rangle
$$

Zur Bestimmung des Interferenzmusters werden einfallende und reflektierte Welle überlagert und das Betragsquadrat gebildet (unter Berücksichtigung der aus dem Kramerschen Theorem abgeleiteten Orthogonalitäten):

$$
\begin{aligned}
\left|\psi_{\text {in }}+\psi_{\text {out }}\right|^{2}=\left|\psi_{\text {in }}\right|^{2}+\left|\psi_{\text {out }}\right|^{2} & \\
& +\left(\mathrm{A}_{1}^{*} \mathrm{~A}_{4}+\mathrm{A}_{1} \mathrm{~A}_{4}^{*}\right) \cdot \operatorname{Re}\left\langle\overrightarrow{\mathrm{k}}_{1}, \uparrow \mid-\overrightarrow{\mathrm{k}}_{2}, \uparrow\right\rangle \\
& +\left(\mathrm{A}_{2}^{*} \mathrm{~A}_{3}+\mathrm{A}_{2} \mathrm{~A}_{3}^{*}\right) \cdot \operatorname{Re}\left\langle\overrightarrow{\mathrm{k}}_{2}, \downarrow \mid-\overrightarrow{\mathrm{k}}_{1}, \downarrow\right\rangle .
\end{aligned}
$$

Die Auswertung der Interferenzterme führt zu einer stehenden Welle, welche die Wellenzahl $\mathrm{k}_{1}+\mathrm{k}_{2}$ besitzt. Führt man eine Auftragung $\varepsilon\left(\mathrm{k}_{\text {STM }}\right)$ mit $\mathrm{k}_{\mathrm{STM}}=\left(\mathrm{k}_{1}+\mathrm{k}_{2}\right) / 2$ durch, dann ergibt dies eine Parabel, welche mittig zwischen den beiden spinaufgespaltenen Parabeln in Abb. 6.6 liegt. Das Interferenzmuster ist also invariant gegenüber einer Spin-Bahn-Aufspaltung der Dispersionsrelation. Die Spin-Bahn-Aufspaltung kann deshalb prinzipiell nicht mit den hier verwendeten STM- Methoden beobachtet werden. 


\subsection{Die Au(111)-Rekonstruktion}
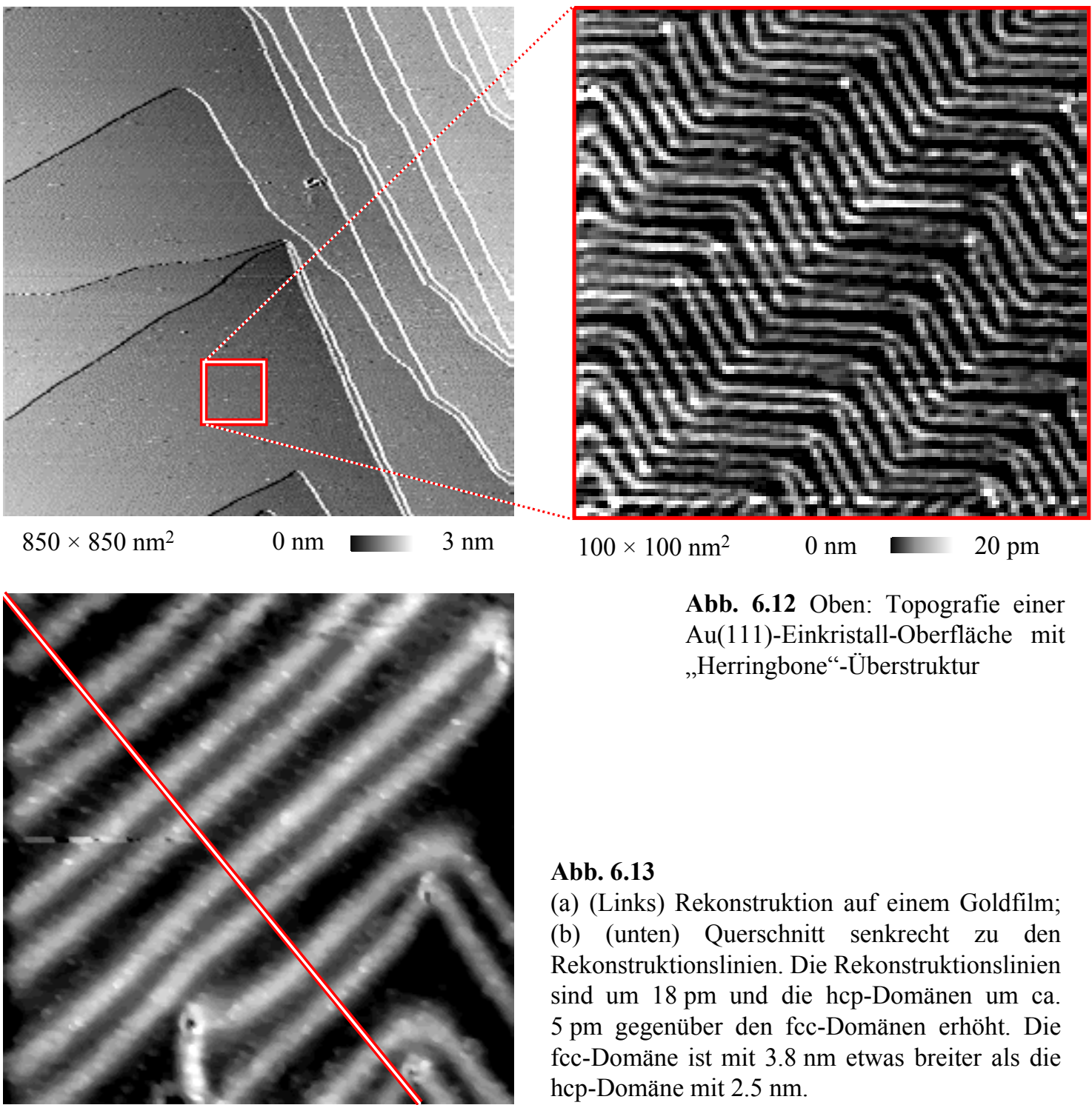

Abb. 6.12 Oben: Topografie einer $\mathrm{Au}(111)$-Einkristall-Oberfläche mit „Herringbone“-Überstruktur

\section{Abb. 6.13}

(a) (Links) Rekonstruktion auf einem Goldfilm; (b) (unten) Querschnitt senkrecht zu den Rekonstruktionslinien. Die Rekonstruktionslinien sind um $18 \mathrm{pm}$ und die hcp-Domänen um ca. 5 pm gegenüber den fcc-Domänen erhöht. Die fcc-Domäne ist mit $3.8 \mathrm{~nm}$ etwas breiter als die hcp-Domäne mit $2.5 \mathrm{~nm}$.

$$
27 \times 27 \mathrm{~nm}^{2} \quad 0 \mathrm{~nm} \quad 20 \mathrm{pm}
$$

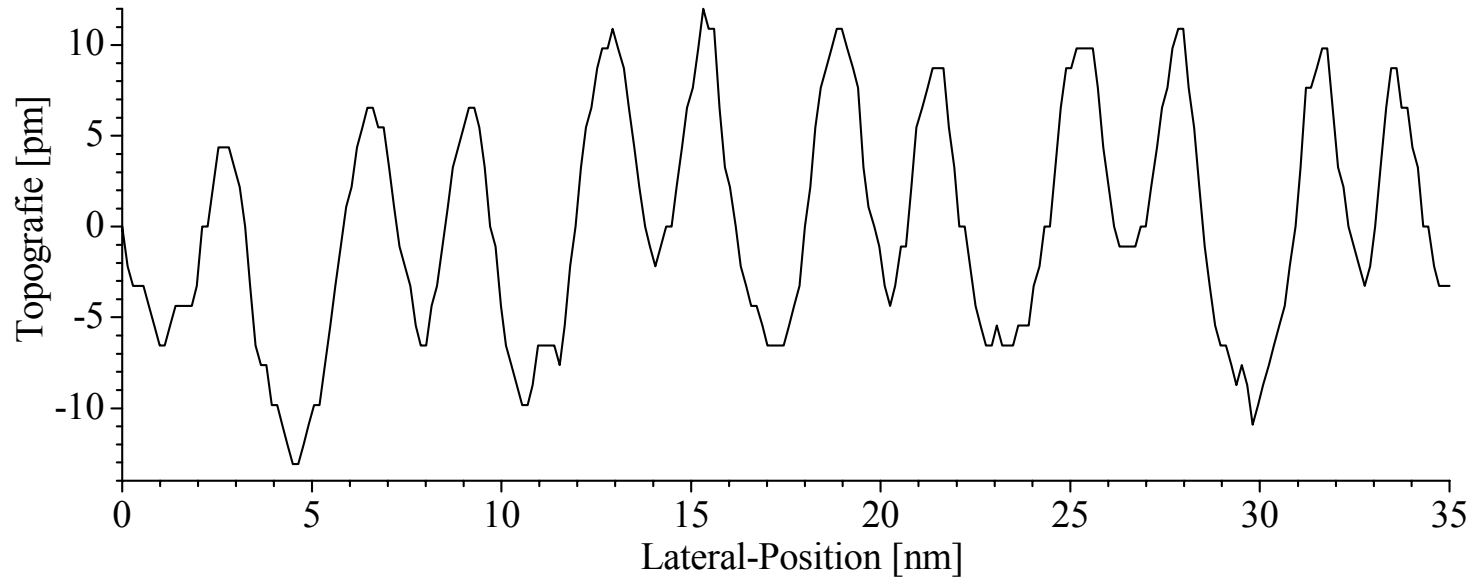


Die Au(111)-Oberfläche weist ein charakteristisches topografisches Muster, die sog. „Herringbone“-Überstruktur, auf [Bar 4, Fuj 4, Har, Wöl]. Wie in Abb. 6.12 zu sehen ist, besteht diese Überstruktur aus benachbarten Doppelreihen, welche auf einem Einkristall sägezahnartig ca. alle $25 \mathrm{~nm}$ kollektiv ihre Richtung um $60^{\circ}$ ändern. Die in den Abbildungen hell erscheinenden Rekonstruktionslinien trennen jeweils zwei Domänen mit unterschiedlicher Stapelfolgen der obersten Atomlage voneinander. In einer Domäne setzen die Atome die kubisch flächenzentrierte Stapelung ( $\mathrm{fcc}$, ...ABCABC) des Kristalls fort, in der anderen Domäne werden die Zwischengitterplätze mit der hexagonal dichtesten Kugelpackung (hcp, ...ABCABA) besetzt. Die Rekonstruktionslinien ${ }^{[7]}$ bilden einen Übergangsbereich zwischen hcp- und fcc-Domänen und erscheinen in der Topografie erhöht, da die Atome weder den Minima der fcc- noch denen der hcp-Stapelplätzen zugeordnet sind und deshalb in Richtung der Oberflächennormalen ausweichen (Abb. 6.13 (b)).

Auf einem Goldfilm kann der Verlauf der Rekonstruktionslinien auch komplexere Formen annehmen, wie in Abb. 6.14 dargestellt ist. Die Ursache hierfür ist in leichten Verspannungen der Oberfläche zu suchen, wie sie z.B. aufgrund des Korngefüges bei Goldfilmen vorhanden sein können.

Zumeist überqueren Rekonstruktionslinien monatomare Stufen, ohne ihre Richtung zu ändern.
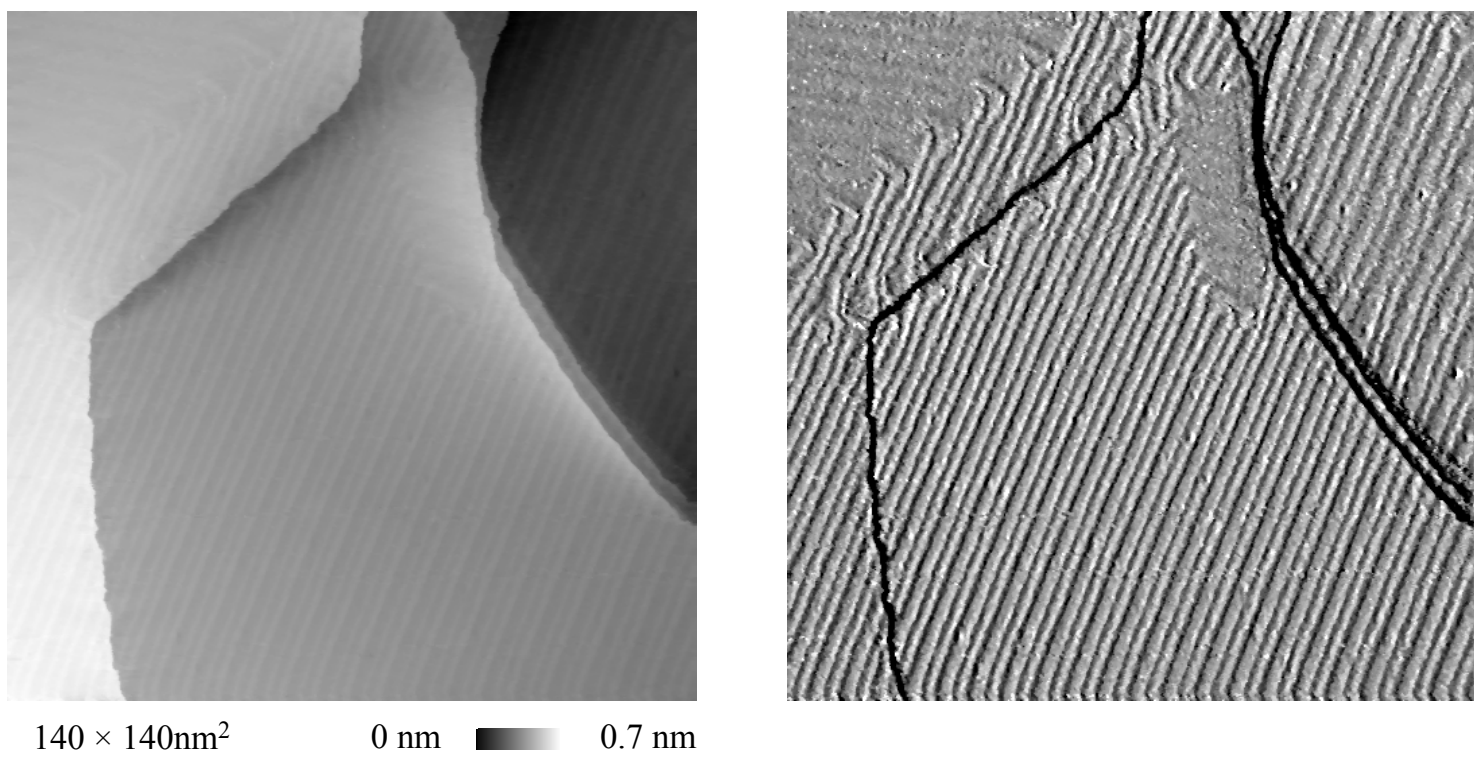

Abb. 6.14 Rekonstruktionsmuster auf einem Goldfilm. Links: Rohdaten Topografie, rechts: Ableitung der Topografie in $\mathrm{x}$-Richtung. In dieser Darstellungsform werden topografische Änderungen in $\mathrm{x}$ Richtung sehr kontrastreich dargestellt. Rekonstruktionslinien, welche in der oberen Hälfte in x-Richtung verlaufen, werden im differentiellen Bild schwächer dargestellt.

\footnotetext{
${ }^{17}$ In der Literatur werden diese Rekonstruktionslinien häufig als „Solitonen-Wälle“ vom Frenkel-Kontorova-Typ bezeichnet. Als „Soliton-Wall“ versteht man den inkommensurablen Übergangsbereich zwischen zwei kommensurablen Domänen [Zan]. Dies ist im Falle der Au(111)-Oberfläche strenggenommen nicht korrekt, da die Übergangsregion von fcc auf hep Stapelung nicht scharf abgegrenzt ist, bzw. die Kontraktion in $[1 \overline{1} 0]$ Richtung nahezu gleichmäßig ist [Bar 4].
} 
Dabei tritt eine Wechselwirkung auf, die in der Literatur bisher keine Beachtung fand, aber im letzten Kapitel dieser Arbeit eine zentrale Rolle spielt: Die Stufen werden periodisch durch das Muster der Rekonstruktion moduliert, d.h. die Atome scheinen sich bevorzugt in eine der beiden Stapelungsregionen (die hcp-Region) umzuordnen.

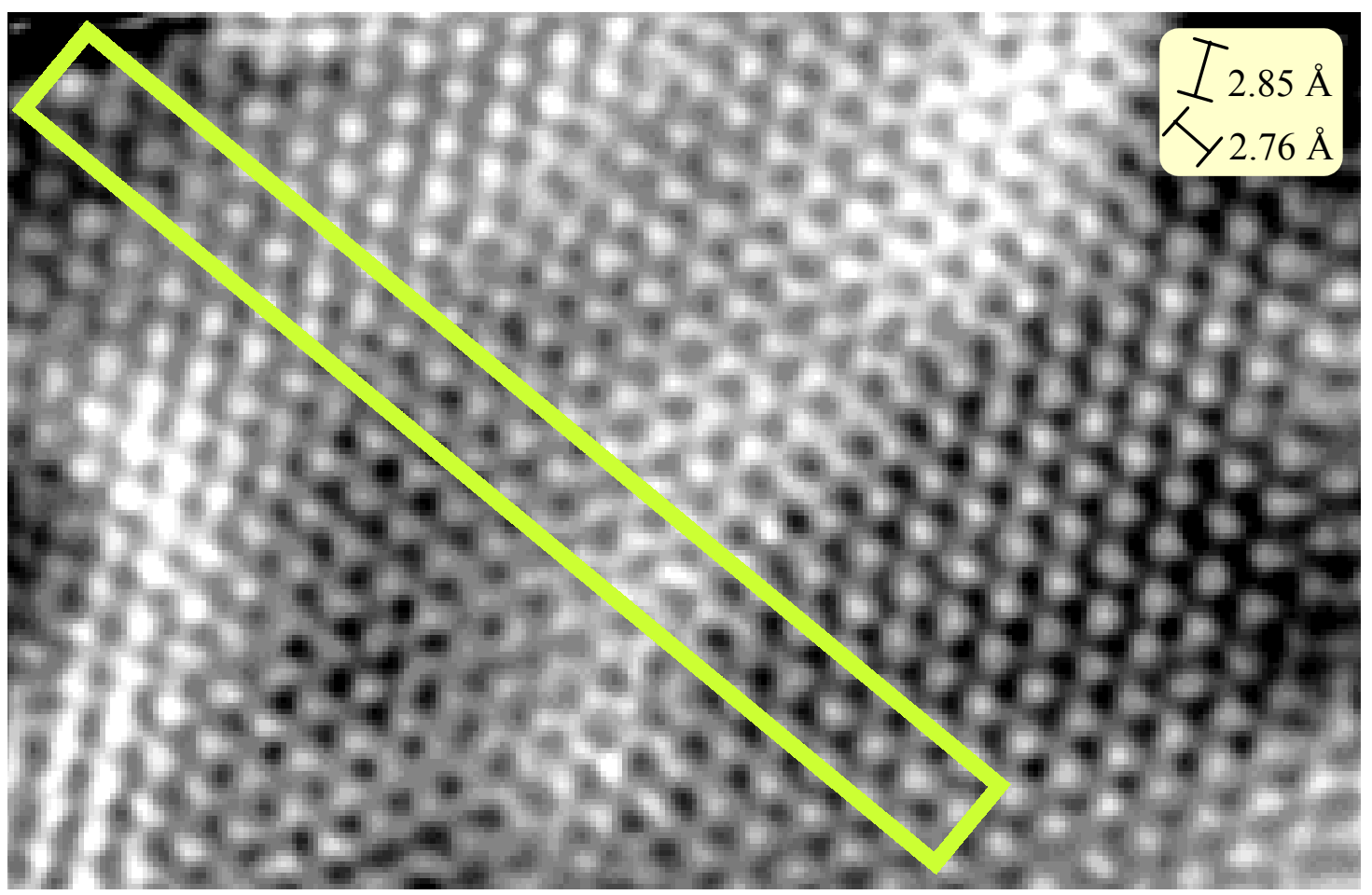

Abb. 6.15 Experimentell erhaltene Atomkarte der Rekonstruktion. $4.5 \mathrm{~nm} \times 7.2 \mathrm{~nm} ; 0 \mathrm{~nm} \square 30 \mathrm{pm}$

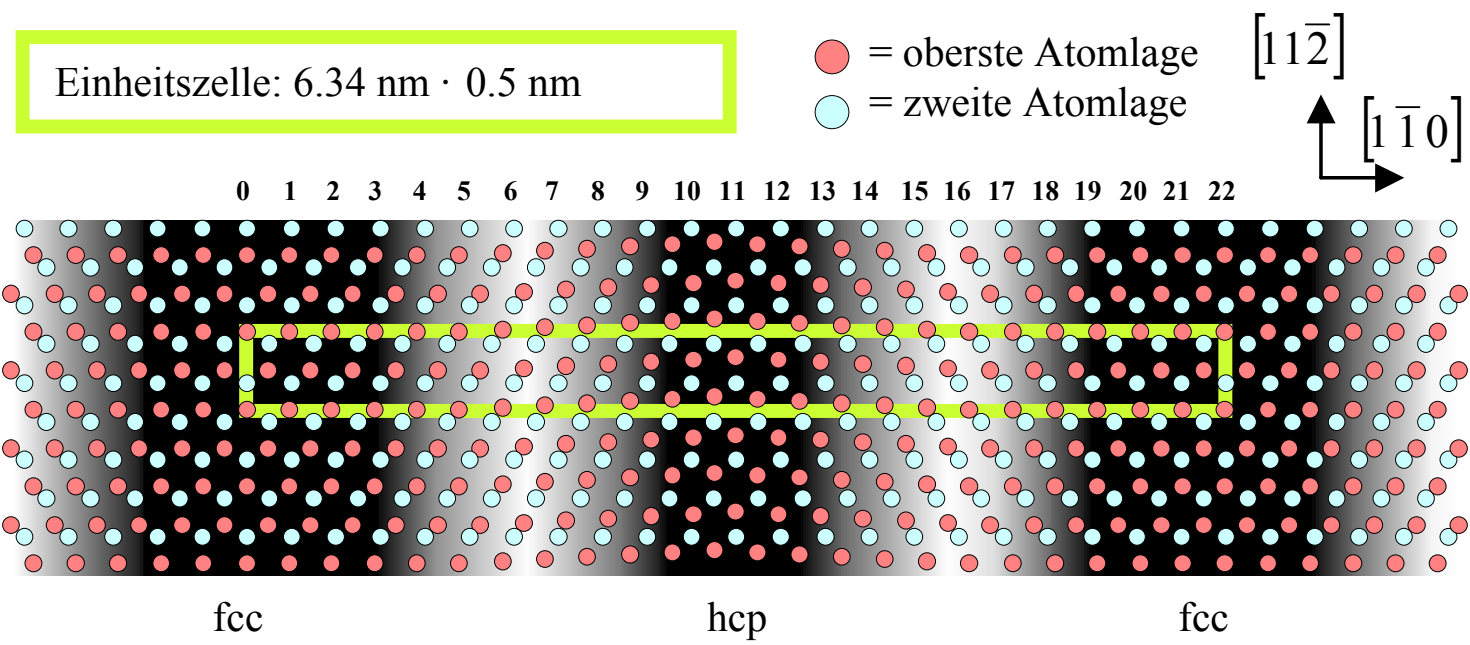

Abb. 6.16 Darstellung der beiden obersten Atomlagen der Rekonstruktion. Die Einheitszelle ist als Rechteck eingezeichnet. 
Für eine Doppelreihe in $[11 \overline{2}]$ - Richtung lässt sich eine rechteckige $22 \times \sqrt{3}$ Elementarzelle 18 angeben, wie sie in Abb. 6.15 und 6.16 dargestellt ist.

Sie enthält auf 22 Volumengitterplätzen 23 Atome in der obersten Lage und stellt eine uniaxiale Kontraktion der Oberfläche um 22/23 senkrecht zu den Rekonstruktionslinien dar. Diese Kontraktion erklärt die Existenz von einer hcp- und einer fcc-Stapelungsdomäne; in der Mitte der in Abb. 6.16 eingezeichneten Elementarzelle ist es für die Atome energetisch günstiger, auf die hcp-Stapelplätze auszuweichen. Dementsprechend sind atomare Reihen senkrecht zu den Rekonstruktionslinien in der hcp-Domäne lateral um $\sqrt{3} / 6 \mathrm{a}=0.083 \mathrm{~nm}$ gegenüber der fcc-Domäne versetzt.

Aufgrund der Symmetrie der Oberfläche gibt es für die Rekonstruktionsreihen drei mögliche Orientierungsrichtungen. Dieser Freiheitsgrad sorgt dafür, dass die anisotrope elastische Verspannung der $22 \times \sqrt{3}$-Rekonstruktion mehr isotropen Charakter erhält, indem die quasieindimensionalen Domänen sägezahnartig gefaltet werden [Nar]. Es bildet sich dadurch die schon erwähnte charakteristische Herringbone-Überstruktur aus.

${ }^{18}$ Die korrekte Bezeichnung für diese Rekonstruktions- Einheitszelle ist durch eine $\left(\begin{array}{cc}22 & 0 \\ -1 & 2\end{array}\right)$-Matrix gegeben, wird im folgenden der Kürze wegen aber (wie auch zumeist in der Literatur) als $22 \times \sqrt{3}$-Matrix angegeben. Die Bezeichnung $22 \times \sqrt{3}$ ist deshalb nicht korrekt, da sich die Richtung der Einheitsvektoren (rechteckige Einheitszelle) gegenüber einer nicht rekonstruierten fcc- (111)-Oberfläche (hexagonale Symmetrie) ändert. 


\title{
7. Interferenz von Elektronenwellen
}

Das zweidimensionale, freie und isotrope Elektronengas der Edelmetall-Oberflächenzustände bietet ein ideales System, in welchem quantenmechanische Interferenzphänomene nicht nur $\mathrm{zu}$ beobachten, sondern auch theoretisch mit vergleichsweise geringem Aufwand zu beschreiben sind. Dazu wird die lokale Zustandsdichte (LDOS) $\rho_{\mathrm{OF}}(\vec{r}, \mathrm{E})$ der Oberflächenzustände berechnet, welche nach Gleichung (2.2) am Ort $\overrightarrow{\mathrm{r}}$ das überlagerte Betragsquadrat aller Wellenfunktionen mit Energie E darstellt.

\subsection{Lokale Zustandsdichte an geradlinigen Stufen}

Bei der Modellannahme einer unendlich langen, geradlinigen Stufe auf der Oberfläche kann eine analytische Lösung für die lokale Zustandsdichte gefunden werden. Man verwendet zweidimensionale ebene Wellen $\psi_{\mathrm{i}}$, welche an der Stufe reflektiert werden, und überlagert die zurücklaufende Welle $\psi_{\mathrm{r}}$ kohärent mit der einlaufenden Welle $\psi_{\mathrm{i}}$.

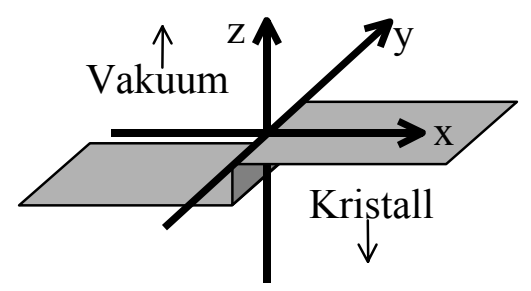

\begin{abstract}
Abb. 7.1 Vereinbarung des Koordinatensystems: Die Stufe führt in $y$-Richtung mit der Oberkante bei $x=z=0$. Die Reflexion der ebenen Welle wird im positiven Halbraum der $\mathrm{x}$-Achse betrachtet.
\end{abstract}

Zur Vereinfachung werde zunächst angenommen, dass die ankommende Welle bei der Stufe totalreflektiert wird und dabei einen Phasensprung von $-\pi$ erfährt (dies entspricht einem 2DPotenzial $\mathrm{V}(\mathrm{x})=\infty$ für $\mathrm{x}<0$ und $\mathrm{V}(\mathrm{x})=\mathrm{E}_{\Gamma}-\mu_{\mathrm{ec}}$ für $\mathrm{x}>0$; eine allgemeinere Betrachtung für beliebige Reflektivitäten und Phasenverschiebungen ist in Anhang A aufgeführt). Die auf die Stufe zulaufende Welle $\psi_{\mathrm{i}}\left(\overrightarrow{\mathrm{r}}, \overrightarrow{\mathrm{k}}_{\|}\right)$besitzt am Ort $\overrightarrow{\mathrm{r}}=(\mathrm{x}, \mathrm{y}, \mathrm{z})$ einen Wellenvektor $\overrightarrow{\mathrm{k}}_{\|}=\left(\mathrm{k}_{\mathrm{x}}, \mathrm{k}_{\mathrm{y}}, 0\right)$ mit $\mathrm{k}_{\mathrm{x}}<0$ :

$$
\psi_{\mathrm{i}}\left(\overrightarrow{\mathrm{r}}, \overrightarrow{\mathrm{k}}_{\|}, \omega\right)=\frac{\psi(\mathrm{z})}{\sqrt{\mathrm{S}_{\mathrm{xy}}}} \cdot \exp \left(\overrightarrow{\mathrm{ik}}_{\|} \overrightarrow{\mathrm{r}}\right)=\frac{\psi(\mathrm{z})}{\sqrt{\mathrm{S}_{\mathrm{xy}}}} \cdot \exp \left(\mathrm{ik}_{\mathrm{x}} \mathrm{x}+\mathrm{ik}_{\mathrm{y}} \mathrm{y}\right)
$$

$\psi(\mathrm{z})$ beschreibt den sowohl zur Vakuumseite wie auch zum Kristallvolumen hin exponentiell gedämpften Anteil der Wellenfunktion. $S_{x y}$ ist das Normierungsvolumen parallel zur Oberfläche. Die reflektierte Welle behält ihre $\mathrm{k}_{\mathrm{y}}$-Komponente bei, aber das Vorzeichen der $\mathrm{k}_{\mathrm{x}}$-Komponente ändert sich: 


$$
\psi_{\mathrm{r}}\left(\overrightarrow{\mathrm{r}}_{,}, \overrightarrow{\mathrm{k}}_{\|}\right)=-\frac{\psi(\mathrm{z})}{\sqrt{\mathrm{S}_{\mathrm{xy}}}} \cdot \exp \left(-\mathrm{ik}_{\mathrm{x}} \mathrm{x}+\mathrm{i \textrm {k } _ { \mathrm { y } } \mathrm { y } )}\right.
$$

Die Überlagerung dieser beiden Wellen, $\psi=\psi_{\mathrm{i}}+\psi_{\mathrm{r}}$ und die anschließende Bildung des Betragsquadrates ergibt für $\mathrm{x}>0$

$$
\left|\psi\left(\overrightarrow{\mathrm{r}}, \overrightarrow{\mathrm{k}}_{\|}\right)\right|^{2}=\frac{|\psi(\mathrm{z})|^{2}}{\mathrm{~S}_{\mathrm{xy}}} \cdot 2 \cdot\left[1-\cos \left(2 \mathrm{k}_{\mathrm{x}} \mathrm{x}\right)\right] .
$$

Es handelt es sich um eine stehende Welle in $|\psi|^{2}$, welche bisher jedoch nur für einen einzigen Zustand (d.h. für eine Welle in eine durch $\overrightarrow{\mathrm{k}}_{\|}$definierte Raumrichtung) durchgeführt wurde. Die LDOS $\rho_{\mathrm{OF}}(\overrightarrow{\mathrm{r}}, \mathrm{E})$ für die Oberflächenzustände ergibt sich jedoch aus der Summation aller $|\psi|^{2}$ bei der Energie E. Da die Dispersionsrelation isotrop ist, besitzen diese Zustände dieselbe Wellenzahl $\mathrm{k}_{\|}(\varepsilon)=\sqrt{2 \mathrm{~m}^{*}\left(\varepsilon+\varepsilon_{\mathrm{F}}\right)} / \hbar$. Allerdings ist die $\mathrm{k}_{\mathrm{x}}$-Komponente von $\overrightarrow{\mathrm{k}}_{\|}$richtungsabhängig, so dass bei der Summation stehende Wellen mit unterschiedlichen Wellenlängen beitragen. Die Summation ergibt deshalb eine resultierende stehende Welle, welche mit dem Abstand $\mathrm{x}$ von der Stufe abklingt. Nach Anhang A wird die Summation berechnet zu

$$
\rho_{\mathrm{OF}}(\overrightarrow{\mathrm{r}}, \varepsilon)=\rho_{\mathrm{OF}}(\mathrm{z}) \cdot\left(1-\mathrm{J}_{0}\left(2 \cdot \mathrm{k}_{\|}(\varepsilon) \cdot \mathrm{x}\right)\right),
$$

wobei $\mathrm{J}_{0}$ die Besselfunktion erster Gattung nullter Ordnung (s. Abb.7.2 und $\rho_{\mathrm{OF}}(\mathrm{z})=|\psi(\mathrm{z})|^{2} \cdot \mathrm{m}^{*} /\left(\pi \hbar^{2}\right)$ ist. $|\psi(\mathrm{z})|^{2}$ gibt die z-Abhängigkeit der LDOS an und $\mathrm{m}^{*} /\left(\pi \hbar^{2}\right)$ ist die Zustandsdichte für ein zweidimensionales Elektronengas [Ash].

In der Verallgemeinerung für beliebige Amplituden-Reflexionsgrade $\mathrm{R}$ und Phasenverschiebungen $\varphi$ bei der Reflexion der Welle ergibt sich nach Gleichung (A.11), Anhang A eine LDOS, welche qualitativ die Form einer um $\varphi$,phasenverschobenen“ Besselfunktion besitzt. Aus Gründen der Übersichtlichkeit wird im folgenden eine feste Phasenverschiebung von $\varphi=-\pi$ angenommen, für welche sich die LDOS zu

$$
\rho_{\mathrm{OF}}(\overrightarrow{\mathrm{r}}, \mathrm{E})=\frac{\mathrm{m}^{*}}{\pi \hbar^{2}} \cdot|\psi(\mathrm{z})|^{2} \cdot\left(1-\mathrm{R} \cdot \mathrm{J}_{0}\left(2 \mathrm{k}_{\|} \mathrm{x}\right)\right)
$$

vereinfacht. Die in dieser Arbeit vorgestellten Rechnungen können prinzipiell mit der im Anhang A aufgeführten allgemeineren LDOS durchgeführt werden; außer einer deutlich umständlicheren Darstellung der Formeln würden jedoch keine neuen fundamentale Erkenntnisse gewonnen. 


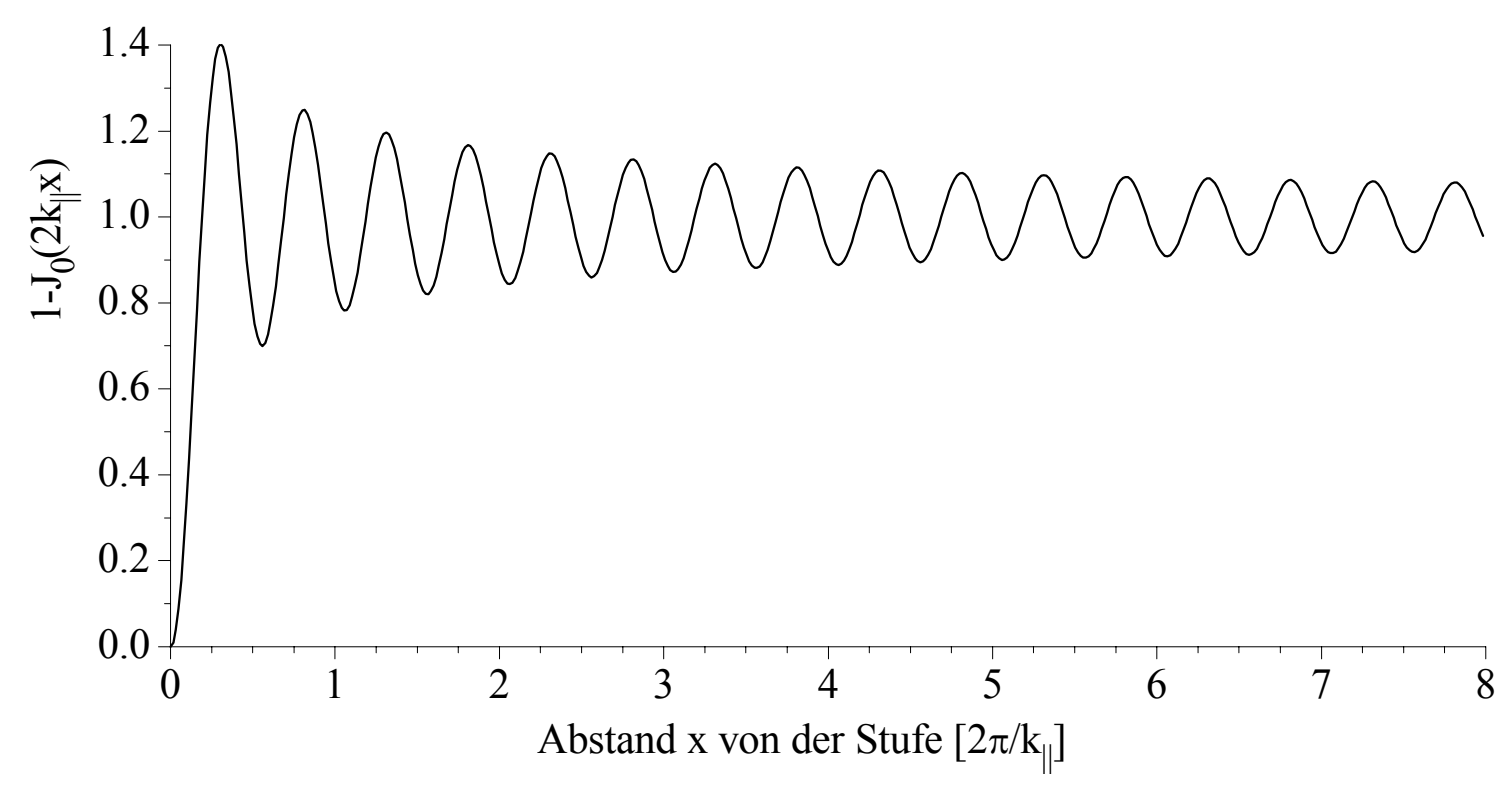

Abb. 7.2 Abstandsabhängigkeit der lokalen Zustandsdichte (,stehende Welle“) an einer geradlinigen Oberflächenstufe bei $\mathrm{x}=0$. Überlagert werden nur Wellenfunktionen mit konstanter Energie $\varepsilon$. Die stehende Welle klingt mit dem Abstand ab, da zwar der Wellenvektorbetrag $\mathrm{k}_{\|}(\varepsilon)$ der überlagerten Wellen konstant ist, nicht jedoch deren $\mathrm{x}$-Komponente $\mathrm{k}_{\mathrm{x}}$. Die $\mathrm{x}$-Achse ist in Einheiten der Wellenlänge $2 \pi / \mathrm{k}_{\|}$ gewählt.

\subsection{Lokale Zustandsdichte für allgemeinere Geometrien}

Ein anderer Ansatz zur Berechnung von Interferenzmustern wurde von D. M. Eigler und Mitarbeitern [Hel 1] verwendet. Die Spitze wird hier als „Sender“ (bzw. als „Empfänger“) einer Kugelwelle mit Amplitude $\mathrm{a}_{\mathrm{T}}(\overrightarrow{\mathrm{r}})$ betrachtet. Die Kugelwelle propagiert von der Spitze aus entlang der Oberfläche, bis sie auf ein (punktförmiges) Streuzentrum am Ort $\vec{r}_{j}$ trifft. Dieses emittiert bei der Streuung wiederum eine Kugelwelle mit Amplitude $a_{T}\left(\vec{r}_{j}\right) \cdot a_{j}(\vec{r})$, welche ihren Weg zurück zur Spitze findet und sich kohärent mit der Primärwelle überlagert (s. Abb. 7.3.

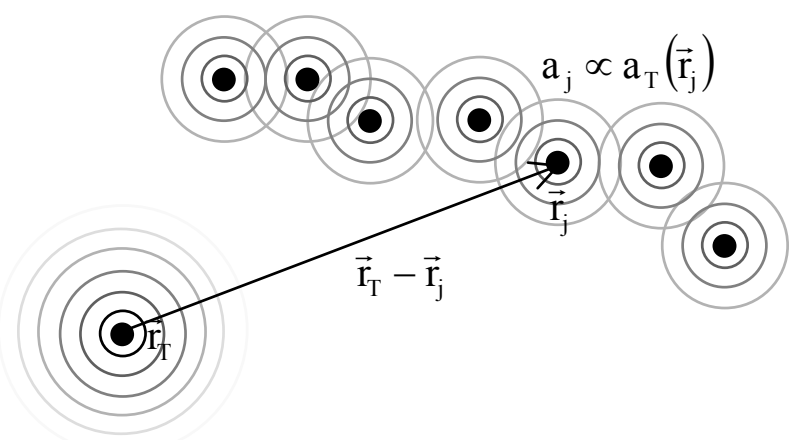

Abb. 7.3 Schemazeichnung zum Berech-
nungsverfahren der LDOS nach [Hel 1] für
allgemeine Streugeometrien. Von der
Spitze geht eine Kugelwelle mit Amplitu-
de $\mathrm{a}_{\mathrm{T}}$ aus, welche von punktförmigen
Streuern in Form von Kugelwellen mit
Amplitude $\mathrm{a}_{\mathrm{j}}$ reflektiert wird. 
Wenn die Spitze sich am Ort $\overrightarrow{\mathrm{r}}_{\mathrm{T}}$ befindet, dann kann für große Abstände $\mathrm{k}_{\|} \cdot\left|\overrightarrow{\mathrm{r}}_{\mathrm{T}}-\overrightarrow{\mathrm{r}}\right| » 1$ die „Sender“-Kugelwelle näherungsweise ${ }^{19}$ beschrieben werden zu

$$
\mathrm{a}_{\mathrm{T}}\left(\overrightarrow{\mathrm{r}}, \mathrm{k}_{\|}\right)=\sqrt{\frac{2}{\pi \mathrm{k}_{\|} \cdot\left|\overrightarrow{\mathrm{r}}_{\mathrm{T}}-\overrightarrow{\mathrm{r}}\right|}} \cdot \exp \left(\mathrm{ik}_{\|} \cdot\left|\overrightarrow{\mathrm{r}}_{\mathrm{T}}-\overrightarrow{\mathrm{r}}\right|\right)
$$

Die von Streuzentrum emittierte Kugelwelle ist gegeben zu

$$
\mathrm{a}\left(\overrightarrow{\mathrm{r}}, \mathrm{k}_{\|}\right)=\sqrt{\frac{2}{\pi \mathrm{k}_{\| \mid} \cdot\left|\overrightarrow{\mathrm{r}}_{\mathrm{j}}-\overrightarrow{\mathrm{r}}\right|}} \cdot \exp \left(\mathrm{ik}_{\|} \cdot\left|\overrightarrow{\mathrm{r}}_{\mathrm{j}}-\overrightarrow{\mathrm{r}}\right|\right) \cdot \frac{\exp (\mathrm{i} \varphi)-1}{2 \mathrm{i}}
$$

wobei $\varphi$ die Phasenverschiebung der emittierten gegenüber der Primärwelle angibt. Wenn sich die Spitze am Ort $\vec{r}$ befindet, dann wird die von einem einzelnen Streuzentrum am Ort $\vec{r}_{j}$ zurückreflektierte Amplitude zu

$$
a_{T}\left(\left|\vec{r}_{j}-\vec{r}\right|, k_{\|}\right) \cdot a\left(\left|\vec{r}-\vec{r}_{j}\right|, k_{\|}\right)=\frac{1}{\pi k_{\|}\left|\vec{r}-\vec{r}_{j}\right|} \cdot \exp \left(2 \mathrm{ik}_{\|}\left|\vec{r}-\vec{r}_{j}\right|\right) \cdot \frac{\exp (i \varphi)-1}{2 i}
$$

berechnet. Bei mehreren Streuzentren bei $r_{1}, \ldots, r_{n}$ ist nach [Hel 1] die lokale Zustandsdichte $\rho_{\text {streu }}(\vec{r}, E)$ der Streuzustände proportional zur aufsummierten Amplitude,

$$
\rho_{\text {streu }}(\vec{r}, E) \propto 2 \cdot \sum_{k_{\|}} \operatorname{Re}\left(\sum_{j=1}^{n} a_{T}\left(\left|\vec{r}-\vec{r}_{j}\right|, k_{\|}\right) \cdot a\left(\left|\vec{r}-\vec{r}_{j}\right|, k_{\|}\right)\right)
$$

wobei hier Mehrfachstreuung vernachlässigt wurde. Die Summation über alle Wellenvektoren $\overrightarrow{\mathrm{k}}_{\|}$ergibt die gesamte lokale Zustandsdichte zu

$$
\rho_{\mathrm{OF}}(\overrightarrow{\mathrm{r}}, \mathrm{E})=\frac{\mathrm{m}^{*}}{\pi \hbar^{2}} \cdot|\psi(\mathrm{z})|^{2} \cdot\left(1-2 \cdot \operatorname{Re}\left[\sum_{\mathrm{j}=1}^{\mathrm{n}} \mathrm{a}_{\mathrm{T}}\left(\left|\overrightarrow{\mathrm{r}}-\overrightarrow{\mathrm{r}}_{\mathrm{j}}\right|, \mathrm{k}_{\|}\right) \cdot \mathrm{a}\left(\left|\overrightarrow{\mathrm{r}}-\overrightarrow{\mathrm{r}}_{\mathrm{j}}\right|, \mathrm{k}_{\|}\right)\right]\right) .
$$

\footnotetext{
${ }^{19}$ Die Einhüllende der Kugelwellenfunktion entspricht der Einhüllenden einer Besselfunktion $\mathrm{J}_{0}$ nullter Ordnung [Cro2]. Am „Emissionsort“ der Kugelwelle besitzt diese den Wert $\mathrm{J}_{0}(0)=1$.
} 


\section{Thermospannungs-Oszillationen an geradlinigen Stufen}

\subsection{Experimentelle Ergebnisse}

In diesem Abschnitt werden einige Messungen von kombinierten Topografie- und Thermospannungsmessungen auf $\mathrm{Au}(111)$ vorgestellt. Die Messungen erfolgen zum einen bei Raumtemperatur und zum anderen bei $\mathrm{LN}_{2}$-gekühlten Proben.

Abb. 8.1 zeigt Messungen, welche bei Raumtemperatur mit einer vom Laser um ca. $4 \mathrm{~K}$ erwärmten Spitze durchgeführt werden. In der Thermospannung ist deutlich ein Muster von stehenden Wellen zu erkennen, welches die Stufen garniert. Der Querschnitt durch die Thermospannung zeigt, dass die Amplitude der Wellen ca. $20 \mu \mathrm{V}$ p-p beträgt und mit zunehmendem Abstand von der Stufe abklingt, bis sie nach ca. $10 \mathrm{~nm}$ im Rauschen von etwa $7 \mu \mathrm{V}$ p-p verschwindet. Die Wellenlänge wird zu $1.8 \mathrm{~nm}$ ausgemessen. Sie entspricht dem halben Wert der Fermi-Wellenlänge $\lambda_{F}$ der Oberflächenzustände. Das Muster kann somit als Interferenzmuster von Elektronenwellen gedeutet werden, welche nach der Reflexion an Stufen ein stehendes Wellenfeld in der lokalen Zustandsdichte erzeugen.

Bei Raumtemperatur wurden stehende Wellen in Thermospannungs-Karten erstmals von D. Hoffmann in der Gruppe um R. Möller beobachtet und in der Folgezeit mittels numerischer Berechnungen charakterisiert [Hof 2-5, Sch 1-2, Hof4].

Zusätzlich zum Interferenzmuster ist ein besonders starkes Thermospannungssignal direkt an Oberflächen-Stufen sichtbar. Dieses Signal hängt nach M. A. Schneider [Sch 1-2] mit der besonderen elektronischen Struktur an Stufenkanten zusammen, bei welcher das Tunneln von Ladungsträgern in erhöhtem Maße über Volumenzustände erfolgt [Avo 2, Avo 5, Li 4]. Die Höhe dieses Signals ist stark von der Konfiguration der Spitze abhängig und erscheint fast immer als betragsmäßig erhöhte Thermospannung [Eng 2, Sch 1-2]. In Abb. 8.1 ist ferner zu erkennen, dass auch vicinale Oberflächen (wie z.B. rechts unterhalb der zentralen Insel) eine ähnliche Erhöhung der Thermospannung wie einzelne Stufen zeigen. 


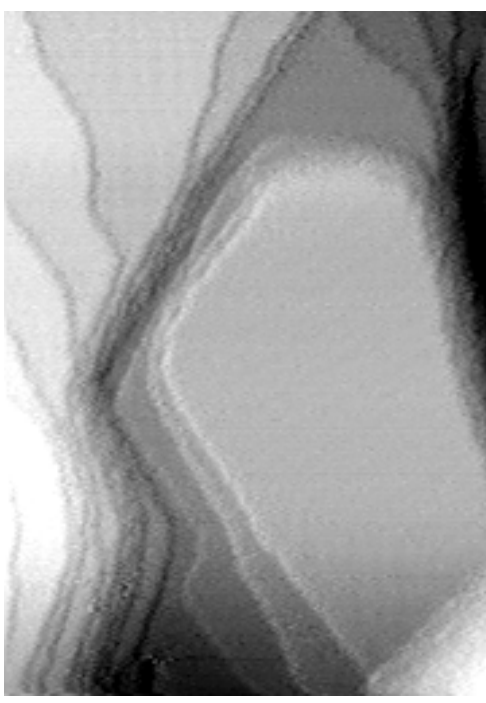

Topografie $0 \mathrm{~nm}$

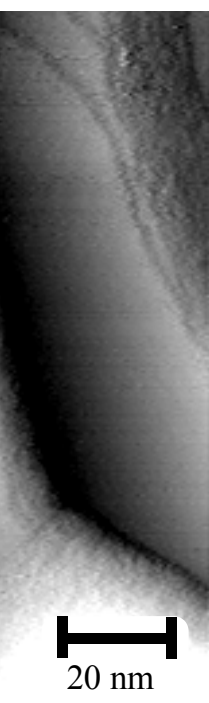

$2 \mathrm{~nm}$

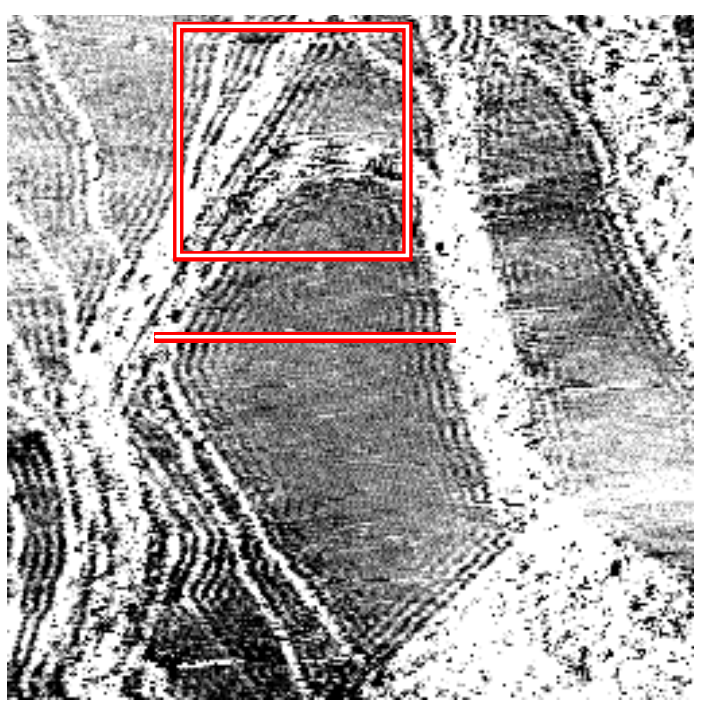

Thermospannung $0 \mu \mathrm{V} \quad 70 \mu \mathrm{V}$

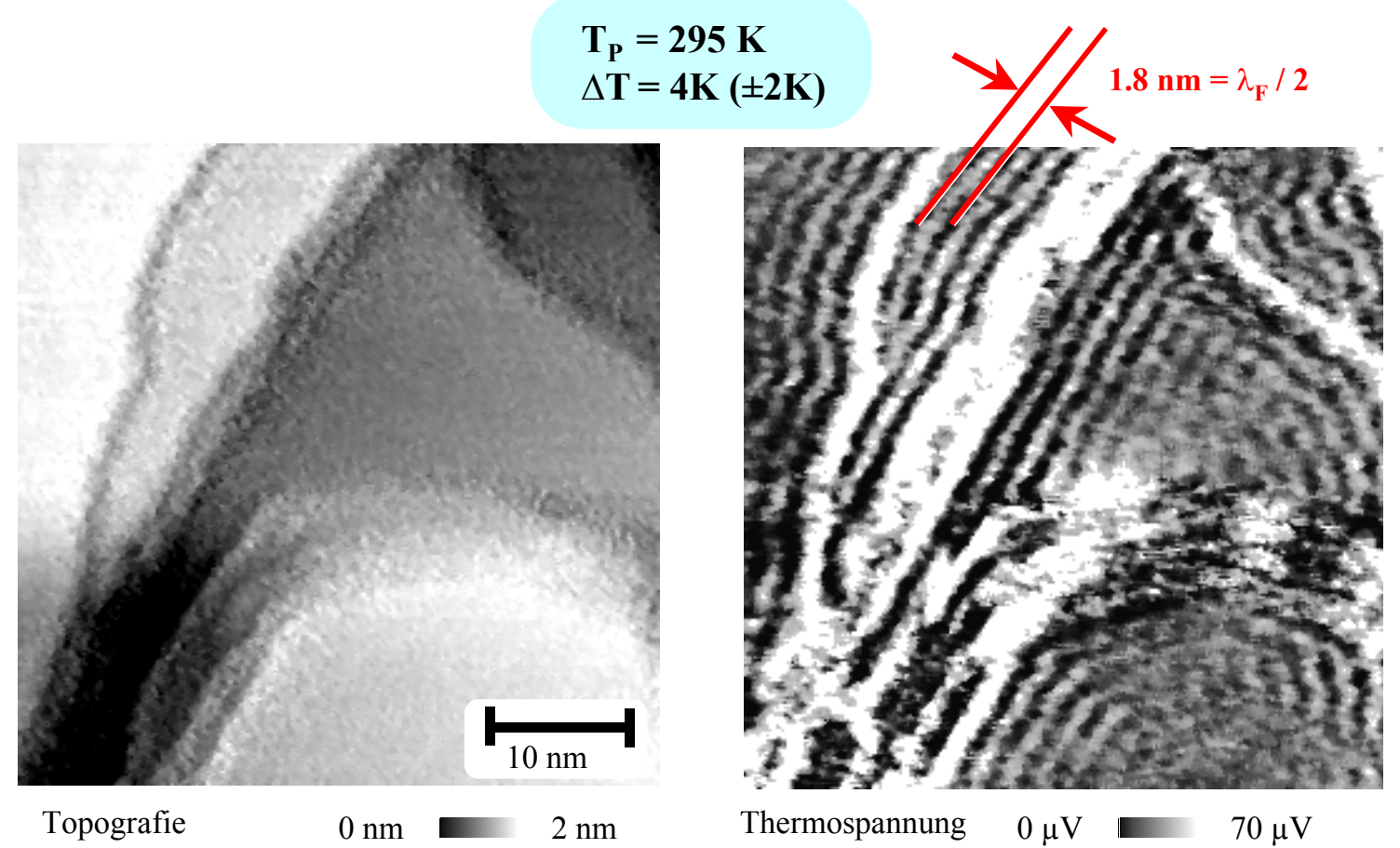

Abb. 8.1 Interferenzmuster von Elektronenwellen, welche an monatomaren Stufen gestreut werden. Die Messung erfolgt bei Raumtemperatur mit lasergeheizter Spitze an einem aufgedampften Goldfilm. Die unteren beiden Messungen stellen einen vergrößerten Ausschnitt der oberen beiden Messungen dar. 


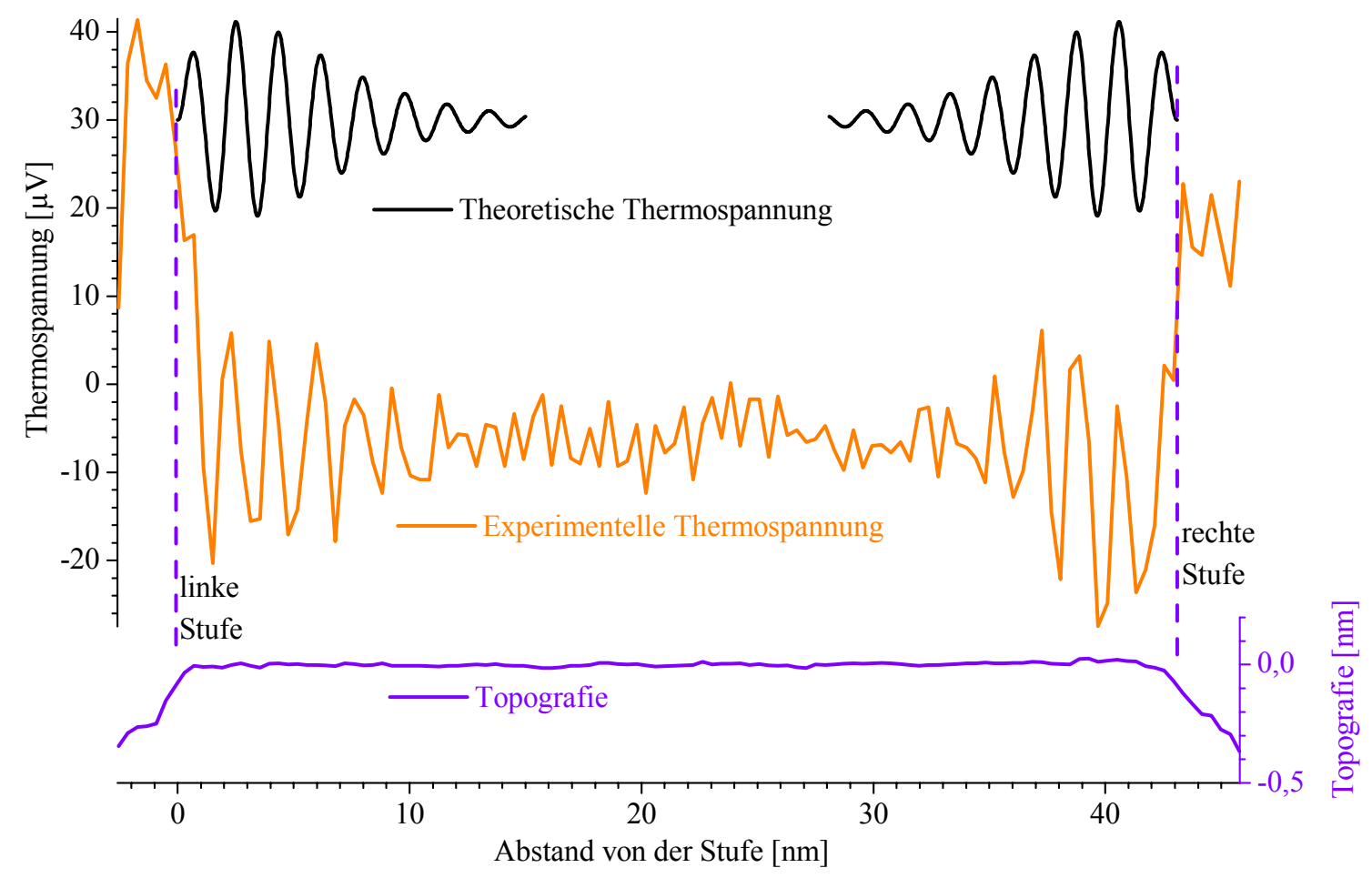

Abb. 8.2 Querschnitte durch die in Abb. 8.1 dargestellte Topografie (-) und Thermospannung (-). Die für eine Kohärenzlänge von $\mathrm{L}_{\varphi}=30 \mathrm{~nm}$ berechnete theoretische Thermospannung (s. Kap. 8.4, unter Vernachlässigung von Elektronen-Confinement durch die Inselform) ist in schwarz eingezeichnet (_).

Bei Raumtemperatur können stehende Wellen bis zu ähnlich großen Abständen von der Stufe nachgewiesen werden, wie sie bereits auch schon mit dI/dU-Spektroskopie beobachtet worden sind [Avo 1-5], Eine deutliche Änderung der Einhüllendenform der stehenden Wellen tritt ein, wenn $\mathrm{Au}(111)$ Oberflächen bei tieferen Temperaturen untersucht werden, wie in Abb. 8.3 gezeigt. Die Messung erfolgt für eine Probentemperatur von ca. $110 \mathrm{~K}$ und einer Temperatur der Spitze von ca. 155 K. Die Topografie zeigt in der Mitte die (111)-Terrasse eines Goldkorns, welche eine Breite von etwa $40 \mathrm{~nm}$ besitzt. In der simultan aufgenommenen Thermospannungsmessung ist ein Interferenzmuster $\mathrm{zu}$ erkennen, welches jedoch im Gegensatz zur Raumtemperaturmessung in Abb. 8.1 auf der gesamten Terrasse zu finden ist. Auffällig ist hier eine gewisse Anisotropie der stehenden Wellen, d.h. es werden nach links bzw. rechts orientierte Wellen mit einer stärkeren Amplitude als in die dazu senkrechte Richtung dargestellt. Besonders ausgeprägt ist die Amplitude im linken unteren Viertel der Insel. Zum einen stößt die Insel an dieser Stelle mit einem anderen leicht verkippten Korn zusammen. Die Korngrenze besitzt vermutlich eine deutlich höhere Reflektivität als eine monatomare Stufe, was sich dann in einer erhöhten Amplitude des stehenden Wellenfeldes äußert. Zum anderen zeigt hier vermutlich die Geometrie der Spitze einen Einfluss. Eine Analyse von Messungen der Stufenkontur mit derselben Spitze an monatomaren Stufen zeigt tatsächlich, dass die Spitze in x-Richtung eine höhere Auflösung besitzt als in y-Richtung. 


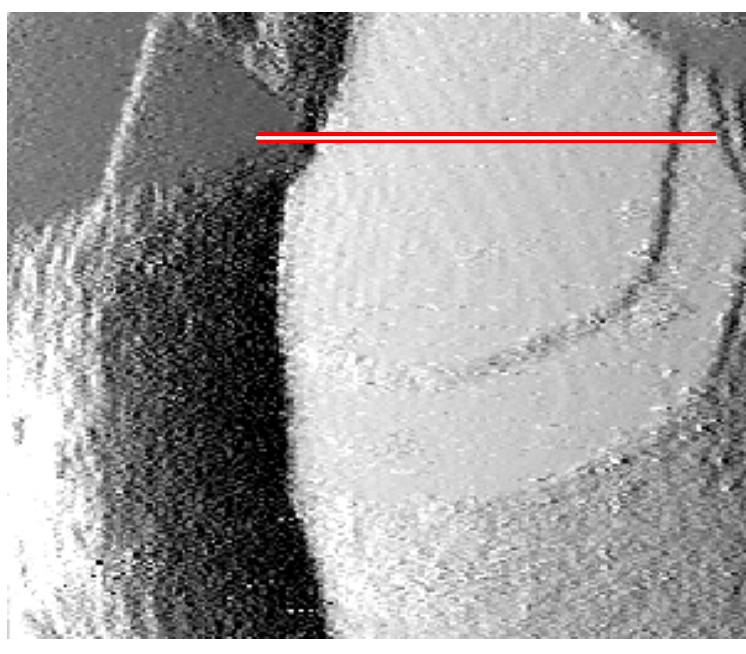

Topografie
$0 \mathrm{~nm}$

$4 \mathrm{~nm}$

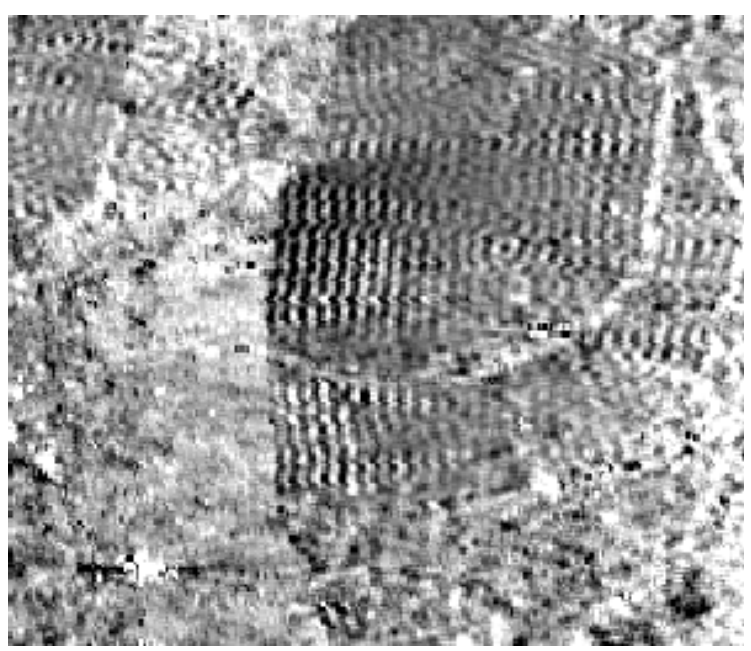

Thermospannung

$0 \mu \mathrm{V}$

$250 \mu \mathrm{V}$

$20 \mathrm{~nm}$

Topografie- und

Thermospannungsmessung bei tiefen Temperaturen an einem Goldkorn mit hoher Korngrenzen-Reflektivität.

$\mathbf{T}_{\mathbf{P}}=\mathbf{1 1 0} \mathbf{K}( \pm \mathbf{3 0} \mathbf{~ K})$
$\Delta \mathbf{T}=\mathbf{4 5} \mathbf{K}( \pm \mathbf{2 0 ~ K})$
Abb. 8.4
Hochaufgelöste reine
Topografiemessung
der obersten Terrasse
des Goldkorns.

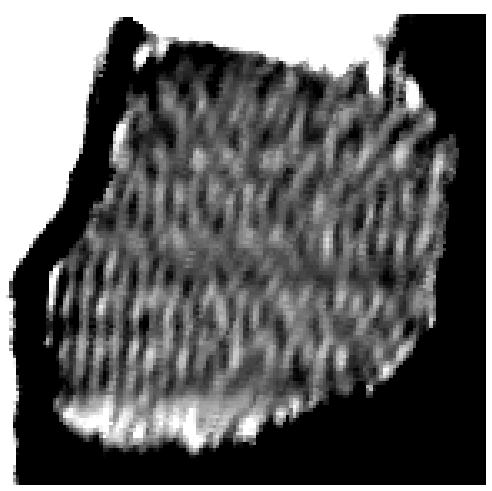

$0 \mathrm{~nm}$

$20 \mathrm{pm}$
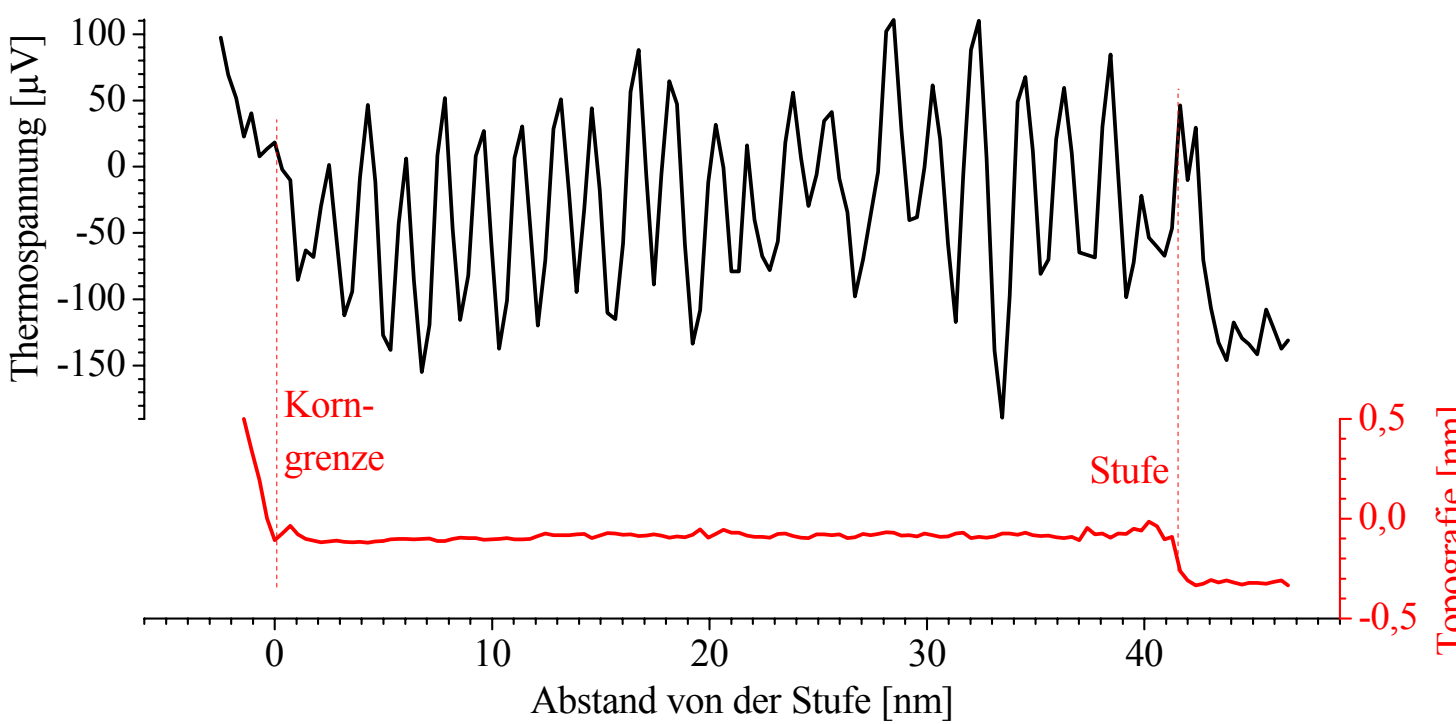

Abb. 8.5 Querschnitt durch Topografie und Thermospannung entlang der Markierung in Abb. 8.3 links oben.

Möglicherweise werden also stehende Wellen in die y-Richtung aufgrund eines Faltungsprozesses unterdrückt. Dieser Effekt zeigt eine Problematik in der Interpretation von Interferenzmustern: die Amplitude von stehenden Wellenfeldern wird von der Auflösung der Spitze mitgeprägt. 
Abb. 8.4 zeigt eine hochaufgelöste Topografieaufnahme derselben Insel, welche ohne simultane Messung der Thermospannung durchgeführt wird. Auch in der Topografie ist eindeutig ein stehendes Wellenfeld zu identifizieren, welches sich hier mit den Konturen der Herringbone-Rekonstruktion überlagert. Ungewöhnlich ist hier die Amplitude der stehenden Wellen von ca. 20 pm p-p. Üblicherweise werden Interferenzerscheinungen in der Topografie erst bei sehr tiefen Temperaturen bei Helium-gekühlten Proben gefunden [Cro 1-5, Che 1, Fuj 1-5 und können ab $80 \mathrm{~K}$ nur bei sehr stabilen Tunnelkontakten auch in der Topografie nachgewiesen werden [Bür 1, Bür 4, Jea, Nag]. Die relativ gute Sichtbarkeit der stehenden Wellen ist eine Folge des thermoelektrischen Effektes, welcher auch auftritt, wenn statt einer Potenziometriemessung nur eine pure Topografiemessung durchgeführt wird. Die Beobachtung von stehenden Wellen in der Topografie wird in Kapitel 9 ausführlicher diskutiert. Die bisher vorgestellten Messungen an aufgedampften Goldfilmen zeigen, dass an diesem Proben-System Messungen nur schwierig zu interpretieren sind, da nur selten freie Terrassen mit Durchmessern größer als 50-100 nm auftreten. Interferenzeffekte werden nicht nur von der reflektierenden Stufe allein bestimmt, sondern sind abhängig von der Topologie der gesamten Terrasse oder Insel. Es ist deshalb wünschenswert, ein System zu untersuchen, bei dem Beeinflussungen des Interferenzmusters von benachbarten Stufen oder Korngrenzen weitestgehend ausgeschlossen werden können. Hierfür bieten sich Einkristalloberflächen an.

Da auch Krümmungen der Stufe das Interferenzmuster beeinflussen, sind für quantitative Untersuchungen der stehenden Wellen die üblicherweise vorgefunden monatomaren Stufen nur bedingt geeignet, da sie fast immer eine Rest-Welligkeit besitzen. Es wird deshalb ein Verfahren der in situ Präparation angewendet, welches auf der Probe geradlinige monatomare Stufen erzeugt. Durch kontrolliertes Eintauchen der Spitze in die Probe werden lokal Versetzungen in den Kristall eingebracht. Diese Versetzungen gleiten bei der Verformung des Materials üblicherweise entlang von niedrig indizierten Ebenen mit dichtester Packung von Atomen [Haa], Bei einem fcc-Kristall wie bei Gold sind dies die vier (111)-Ebenen. Wenn Versetzungen an der Oberfläche austreten, dann hinterlassen sie geradlinige Stufen entlang der Schnittlinien von (111)-Ebenen, bzw. den Kantenlinien im Tetraedermodell [Haa]. Konkret heißt dies, dass durch das Eintauchen der Spitze geradlinige monatomare Stufen in der Umgebung des Kraters erzeugt werden. Ein Beispiel einer solchen Prozedur an einer Einkristalloberfläche ist in Abb. 8.6 zu sehen, wo die Spitze in der oberen rechten Bildecke in die Probe eingetaucht worden ist. An einer der Stufen (s. Markierung in Abb. 8.6 wird eine kombinierte Topografie- und Thermospannungsmessung bei ca. $80 \mathrm{~K}$ Probentemperatur durchgeführt, welche in Abb. 8.7 dargestellt ist. 

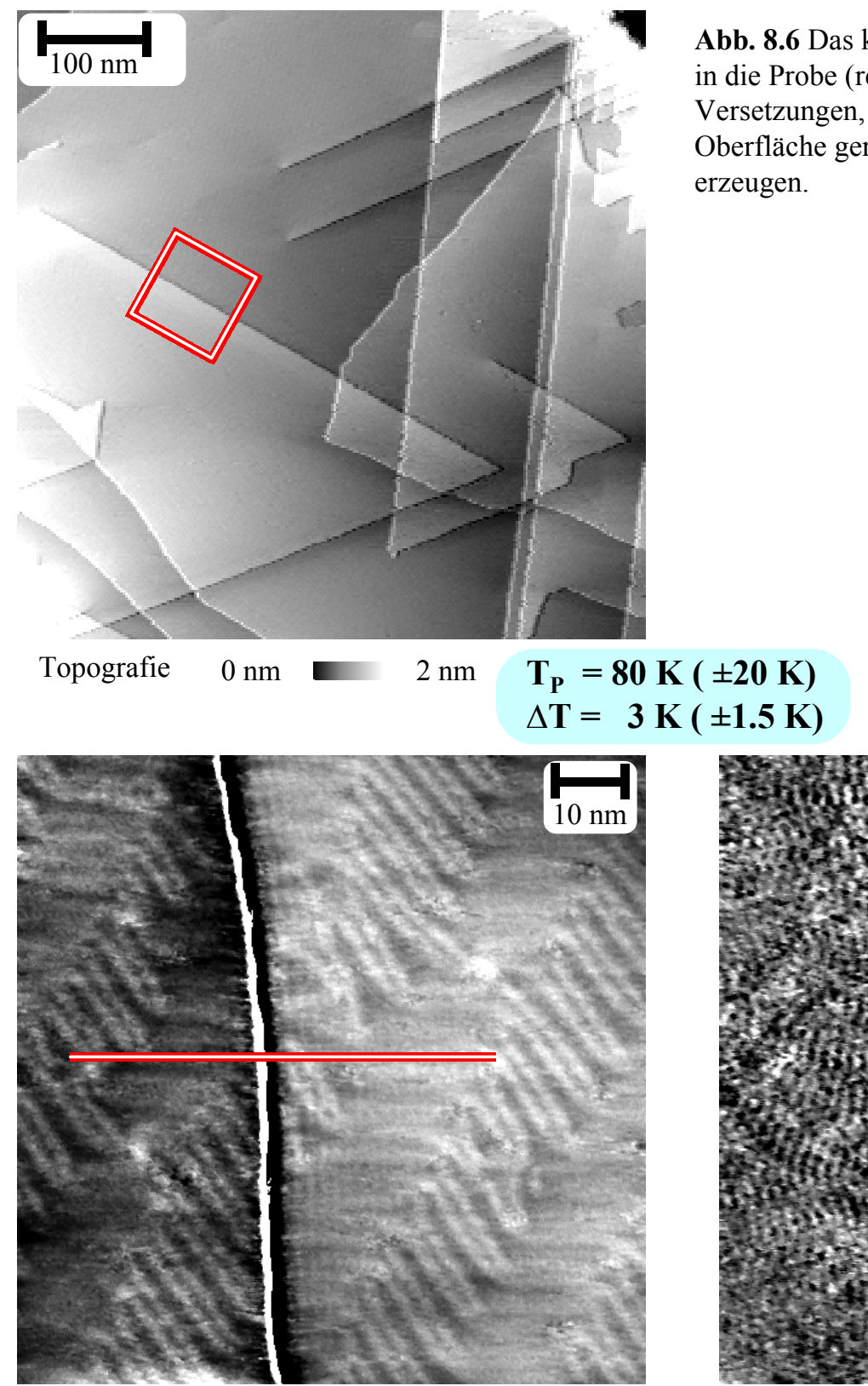

Topografie

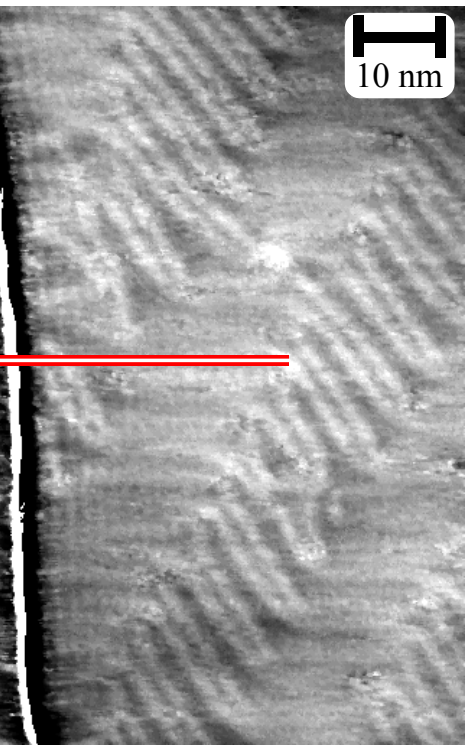

$0 \mathrm{~nm}$

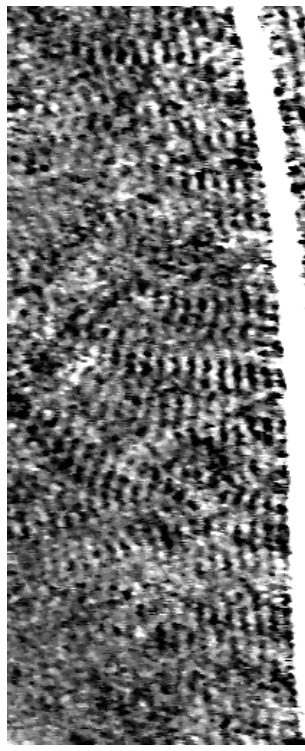

Thermospannung

\begin{abstract}
Abb. 8.7 vergrößerter
Ausschnitt aus Abb. 8.6: kombinierte Topografie- und Thermospannungsmessung an einer geradlinigen Stufe. Die leichte Welligkeit der Stufenkontur ist auf laterale Drift während der Messung zurückzuführen.
\end{abstract}

Auch in dieser Messung sind in der Topografie stehende Wellen in der Nähe der Stufe zu finden, welche jedoch aufgrund der geringeren Temperaturdifferenz verglichen mit Abb. 8.4 deutlich geringer in ihrer Amplitude ausfallen.

In der Thermospannungsmessung ist deutlich ein Muster von stehenden Wellen zu finden, welches noch bis zu einer Entfernung von $30 \mathrm{~nm}$ der Stufe zugeordnet werden kann, wie ein Schnitt durch Topografie und Thermospannung in Abb. 8.8 zeigt. Als Besonderheit kann dem Schnitt entnommen werden, dass die Amplitude der Thermospannungs-Oszillationen mit größer werdendem Abstand von der Stufe zunächst zunimmt, bis sie ein Maximum in einer Entfernung von ca. $10 \mathrm{~nm}$ zur Stufe erreicht, und erst darüber hinaus abklingt. Dieses Verhalten ist sehr ungewöhnlich im Vergleich zu topografischen und spektroskopischen Messungen, welche von der dI/dU-Spektroskopie her bekannt sind. In diesen Messungen wird 
immer ein monotones Abfallen der Oszillationsamplitude festgestellt [SW 1]. Eine ausführliche Diskussion über die Kurvenform wird aufgrund des Umfanges dieser Thematik gesondert im folgenden Abschnitt 8.2 gegeben.

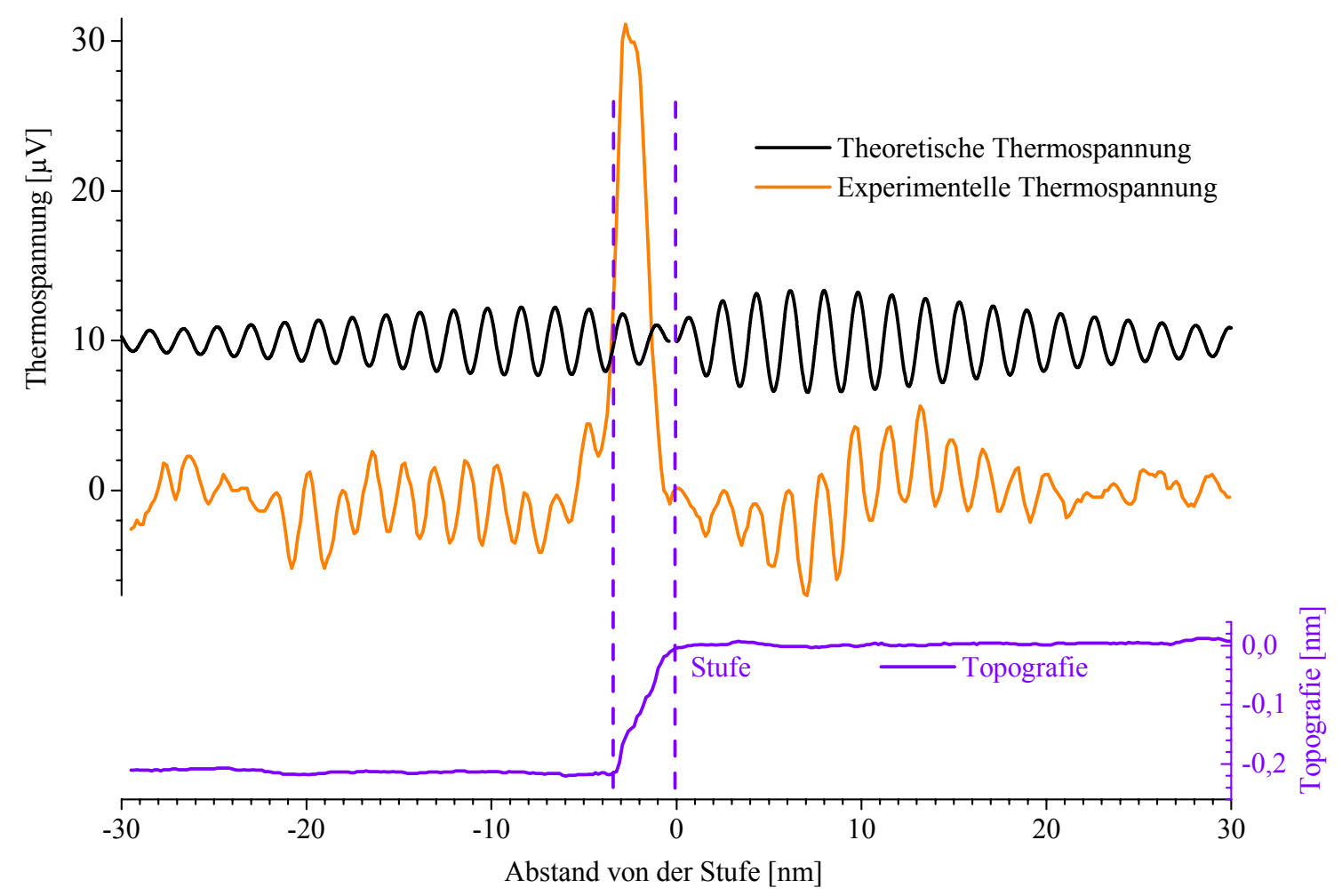

Abb. 8.8 Querschnitt durch Topografie (-) und Thermospannungsmessung (-) entlang der Markierung von Abb. 8.7. Die theoretisch nach Kapitel 8.4 berechnete Thermospannung für eine Kohärenzlänge von $\mathrm{L}_{\varphi}=60 \mathrm{~nm}$ ist in schwarz dargestellt (—).

Das etwa $30 \mu \mathrm{V}$ hohe peak-artige Signal der Thermospannung an der Stufenkante ist auch hier ein Effekt, der von der elektronischen Struktur der Stufenkante herstammt [Sch 1-2]. In Abb. 8.8 ist dieses Signal mit der Topografiekontur der Stufenkante über einen Bereich von ca. $3 \mathrm{~nm}$ korreliert.

Die Reflexion von Elektronenwellen an der Stufe erfolgt sowohl auf der oberen wie auch auf der unteren Terrasse. Die Amplitude der stehenden Wellen ist jedoch auf der oberen Terrasse stärker ausgeprägt als auf der unteren. Nach Kapitel 7.1 ist dies ein Hinweis auf verschiedene Amplitudenreflexionsgrade $\mathrm{R}_{\text {oben }}$ und $\mathrm{R}_{\text {unten }}$ für Elektronenwellen, welche von der oberen bzw. der unteren Terrasse aus auf die Stufe treffen 20 . Aus der Amplituden-Analyse von stehenden Wellen in mehreren Thermospannungskarten wird reproduzierbar ein Verhältnis

\footnotetext{
${ }^{20}$ Der nicht reflektierte Anteil der Elektronenwellen transmittiert zum einen in die Oberflächenzustände auf der jeweils anderen Terrassenseite, zum anderen aber auch in Volumenzustände. Da aus Gründen der Teilchenerhaltung die Transmission Oberflächenzustand $\leftrightarrow$ Oberflächenzustand in beide Richtungen über die Stufe gleich sein muss, stammen die Unterschiede in den Reflexionsgraden von den unterschiedlichen Transmissionsgraden in die Volumenzustände, wenn die Elektronenwellen auf eine aufsteigende bzw. absteigende Stufe treffen.
} 
von $\mathrm{R}_{\mathrm{oben}} / \mathrm{R}_{\mathrm{unten}}=1.5$ gefunden. Andere experimentell gefundene Verhältniswerte sind in Tab. 8.1 zusammengestellt.

\begin{tabular}{|l|l|l|}
\hline $\mathrm{R}_{\text {oben }} / \mathrm{R}_{\text {unten }}$ & Methode & Referenz \\
\hline 5.0 & $\mathrm{dI} / \mathrm{dU}(300 \mathrm{~K})$ & [Has 3] \\
\hline 4.0 & Topografie (30 K) & {$[$ Fuj 3] } \\
\hline 5.0 & SThVM (300 K) & [Hof 4] \\
\hline 1.5 & SThVM (100 K) & Vorliegende Arbeit \\
\hline
\end{tabular}

Tab. 8.1 Experimentell bestimmte Verhältniswerte von Amplituden-

Reflexionsgraden bei auf- bzw. absteigenden Stufen.

Bisherige Literaturwerte geben ein deutlich höheres relatives Amplitudenverhältnis für die stehenden Wellen beiderseits der Stufe an. Die Ursache für diese Diskrepanz ist nicht bekannt.

\subsection{Theoretische Beschreibung der Oszillationen}

In diesem Abschnitt wird die Orts- und Temperaturabhängigkeit der Oszillationen in der Thermospannung nahe einer monatomaren Stufe theoretisch zunächst unter Vernachlässigung von Elektron-Elektron- und Elektron-Phonon-Streuung behandelt. Es wird diskutiert, warum die Oszillationsamplitude mit dem Abstand von der Stufe zunächst zunimmt, bis sie ein Maximum erreicht, bevor sie exponentiell gedämpft wird.

Eine systematische Untersuchung der Orts- und Temperaturabhängigkeit des Interferenzmusters in der Thermospannung wurde in der Literatur bisher nicht durchgeführt; lediglich für den Bereich der Raumtemperatur gibt es Simulationsrechnungen [Hof 2-5, Sch 1], welche mit den experimentellen Ergebnissen in Deckung zu bringen sind.

Es wurde bereits versucht, die Thermospannung analytisch mit Hilfe des Støvneng-LipavskýFormalismus (s. Kap. 3.2) [Sto] zu berechnen [Sch 1, Eng 1, Sei]. Dessen Durchführung führt jedoch zu einer Problematik: Die so berechnete Thermospannung zeigt eine divergierende Oszillationsamplitude mit wachsendem Abstand von der Stufe. Dieses Verhalten wird im Experiment nicht beobachtet. Die Støvneng-Lipavský-Näherung ist fernab der Stufe nicht mehr anwendbar, da die notwendige Annahme einer linearen LDOS um $\mathrm{E}=\mu_{\mathrm{ec}}$ nicht mehr gerechtfertigt ist [Hof 5, Eng 1, Sei].

Eine genauere Charakterisierung des Interferenzmusters erfordert eine über den StøvnengLipavský-Formalismus hinausgehende analytische Behandlung. Eine solche wird im Rahmen dieser Arbeit gefunden im folgenden vorgestellt. 


\subsubsection{Grafische Darstellung der LDOS}

Als Grundlage für die Berechnung des Interferenzmusters an einer monatomaren Stufe wird die lokale Zustandsdichte $\rho_{\mathrm{P}}(\overrightarrow{\mathrm{r}}, \varepsilon)$ benötigt, welche bereits in Kapitel 7.1 beschrieben wurde:

$$
\rho_{\mathrm{P}}(\overrightarrow{\mathrm{r}}, \varepsilon)=\rho_{0} \cdot\left[1-\mathrm{C} \cdot \mathrm{J}_{0}\left(2 \cdot \mathrm{k}_{\|}(\varepsilon) \cdot \mathrm{x}\right)\right]
$$

Die Konstante $\mathrm{C}$ gewichtet hier den Interferenzterm der Oberflächenzustände relativ zur LDOS $\rho_{0}$ einer ungestörten, stufenlosen Oberfläche (s. auch Anhang B.

Zur Interpretation der Thermospannung wird nicht nur die Abhängigkeit der LDOS $\rho_{P}$ vom Abstand $\mathrm{x}$ von der Stufe, sondern auch deren Abhängigkeit von der Energie $\varepsilon$ relativ zum elektrochemischen Potenzial benötigt. Diese ist grafisch in Abb. 8.9 in einem Graustufenplot veranschaulicht.

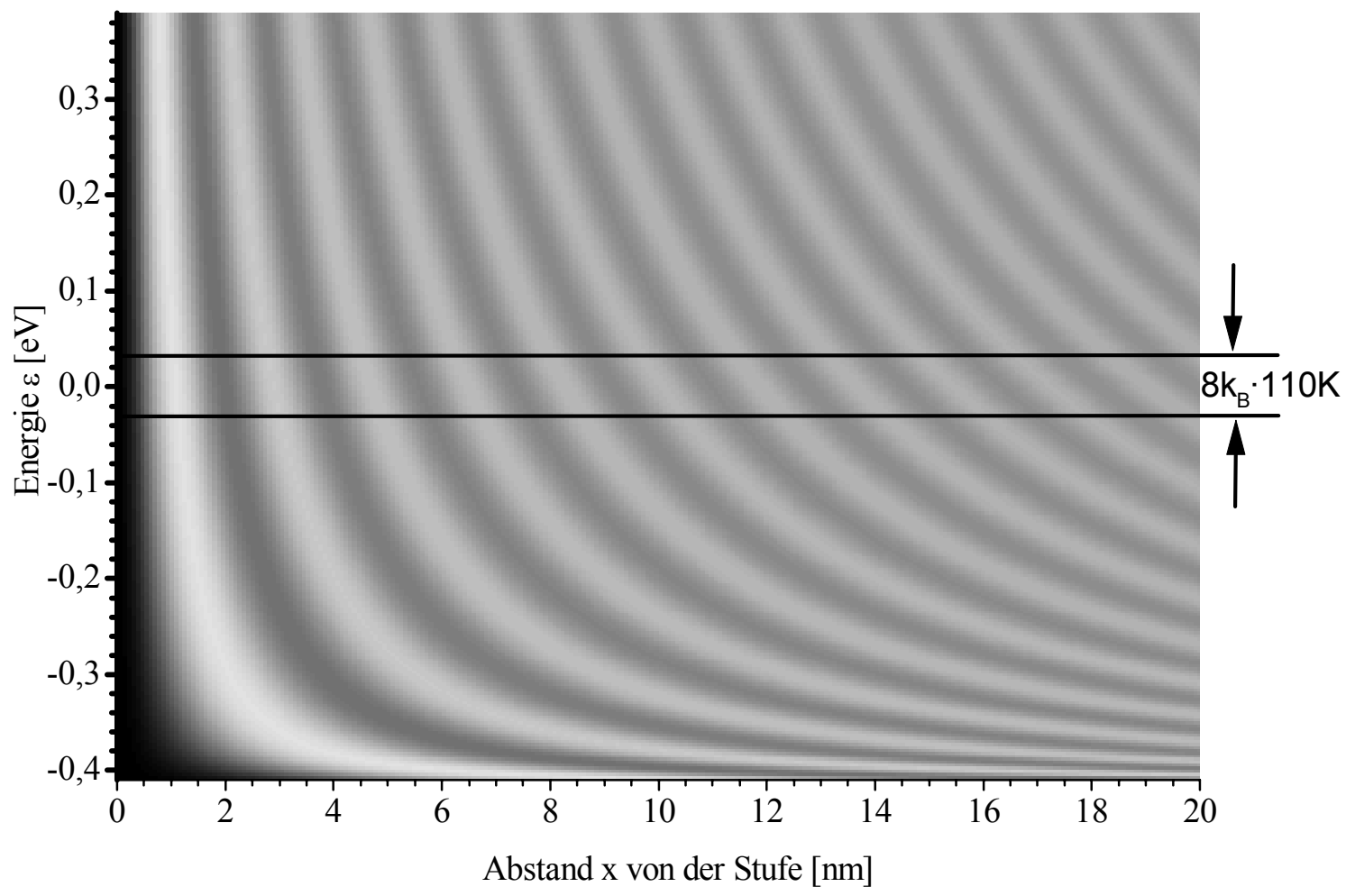

Abb. 8.9 Graustufenkodierte Lokale Zustandsdichte $\rho_{\mathrm{p}}$ der Oberflächenzustände in Abhängigkeit von der Energie $\varepsilon$ der streuenden Oberflächenzustände sowie dem Abstand x von der Stufe. Dunkle Bereiche geben eine geringe LDOS, helle Bereiche eine hohe LDOS an. Eingezeichnet ist der Bereich thermischer Verbreiterung um $\varepsilon=0$ für eine Temperatur von $110 \mathrm{~K}$.

Da die Wellenzahl der Oberflächenzustände von der Energie abhängt, zeigt die LDOS nicht nur Oszillationen im Ortsraum, sondern auch im Energieraum.

In Abb. 8.9 ist der Bereich der thermischen Verbreiterung der Fermifunktion (ca. $8 \mathrm{k}_{\mathrm{B}} \mathrm{T}$ ) als Beispiel für eine Temperatur von $110 \mathrm{~K}$ dargestellt. Für die Berechnung des thermoelektrischen Effekts ist die Energieabhängigkeit der LDOS innerhalb von diesem Bereich ausschlaggebend. Im Støvneng-Lipavský-Formalismus wird hier die Linearität der 
LDOS in $\varepsilon$ als notwendige Voraussetzung gefordert [Sto]. Wie in Abb. 8.9 zu sehen ist, kann diese Forderung für große Abstände von der Stufe nicht mehr erfüllt werden, da die LDOS der Oberflächenzustände im Energieraum innerhalb von $8 \mathrm{k}_{\mathrm{B}} \mathrm{T}$ oszilliert.

\subsubsection{Analytische Behandlung im Tersoff-Hamann-Modell}

Eine genauere analytische Näherung der Thermospannung $\mathrm{U}_{\mathrm{Th}}(\mathrm{x})$ muss das oszillatorische Verhalten der LDOS berücksichtigen. Die mathematische Behandlung im Tersoff-Hamann Modell ist sehr umfangreich und wird deshalb in den Anhang B verlagert. An dieser Stelle sollen nur die zentralen Ergebnisse referiert und physikalisch interpretiert werden.

Die wesentlichen Näherungsschritte bestehen in einer Taylorentwicklung erster Ordnung für die Zustandsdichte der Spitze, der LDOS der Volumenzustände sowie der Dispersionsrelation bei $\varepsilon=0$. Ferner wird die Besselfunktion in der LDOS für die Oberflächenzustände durch die Linearkombination einer Sinus- und Kosinusfunktion genähert. Für die Gültigkeit dieser Näherungen müssen folgende Bedingungen erfüllt sein:

- $\mathrm{eU}_{\mathrm{Th}} \ll \mathrm{k}_{\mathrm{B}} \mathrm{T}$ (experimentell wird $\mathrm{eU}_{\mathrm{Th}} / \mathrm{k}_{\mathrm{B}} \mathrm{T} \approx 10^{-3}$ gefunden).

- Die Zustandsdichte der Spitze und die LDOS der Volumenzustände müssen innerhalb von $\pm 4 \mathrm{k}_{\mathrm{B}} \mathrm{T}$ um $\varepsilon=0$ nahezu linear in $\varepsilon$ sein. Zusätzlich darf die Zustandsdichte der Spitze nur schwach von der Energie abhängig sein, d.h. im Detail $4 k_{B} T \cdot d \rho_{S} / d \varepsilon \ll \rho_{S}$.

- $4 \mathrm{k}_{\mathrm{B}} \mathrm{T} \ll \varepsilon_{\mathrm{F}}$, d.h. die Fermienergie der Oberflächenzustände muss deutlich größer als die thermische Verbreiterung der Fermifunktionen sein. Für $\operatorname{Au}(111)$ mit $\varepsilon_{\mathrm{F}} \approx 410 \mathrm{meV}$ impliziert diese Bedingung $\mathrm{T} \ll 1200 \mathrm{~K}$.

Die analytisch erhaltene Näherungslösung der Thermospannung ist in Abb. 8.10 zusammengefasst. 


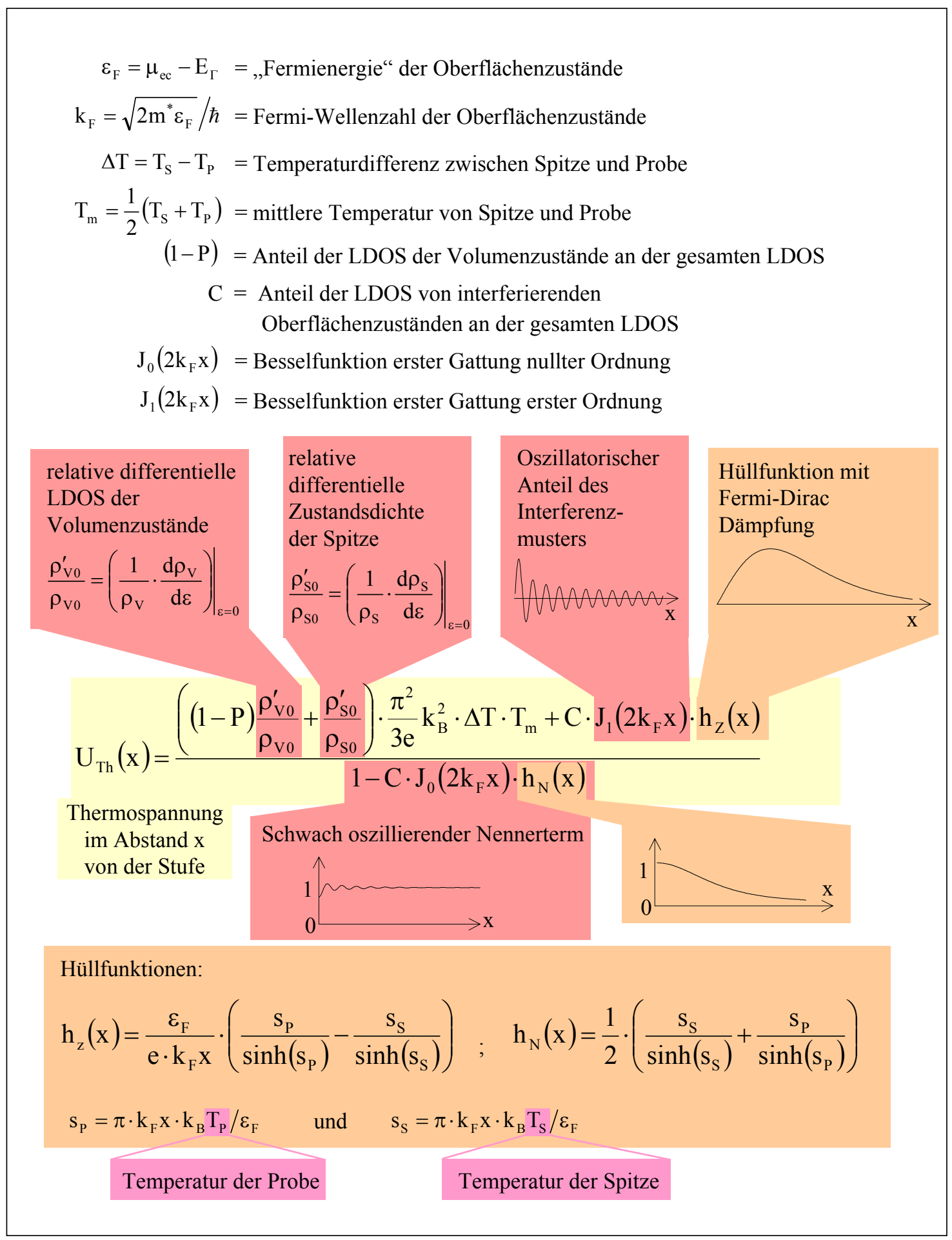

Abb. 8.10 Schematische Darstellung der nach dem Tersoff-Hamann-Modell berechneten Näherungslösung für die Thermospannung $\mathrm{U}_{\mathrm{Th}}(\mathrm{x})$ an einer monatomaren Stufe. 
Zur Vereinfachung der in Abb. 8.10 dargestellten Näherungslösung werde zunächst der Einfluss von der Zustandsdichte der Spitze und der LDOS der Volumenzustände vernachlässigt, indem $\rho_{\mathrm{s} 0}^{\prime} / \rho_{\mathrm{s} 0}=\rho_{\mathrm{v} 0}^{\prime} / \rho_{\mathrm{v} 0}=0$ gesetzt wird (Die Berücksichtigung dieser Terme erfolgt in einem späteren Abschnitt). Die Thermospannung $U_{T h}(x)$ vereinfacht sich dann zu

$$
\mathrm{U}_{\mathrm{Th}}(\mathrm{x})=\frac{\mathrm{C} \cdot \mathrm{J}_{1}\left(2 \mathrm{k}_{\mathrm{F}} \mathrm{x}\right) \cdot \mathrm{h}_{\mathrm{Z}}(\mathrm{x})}{1-\mathrm{C} \cdot \mathrm{J}_{0}\left(2 \mathrm{k}_{\mathrm{F}} \mathrm{x}\right) \cdot \mathrm{h}_{\mathrm{N}}(\mathrm{x})}
$$

mit einer Einhüllendenfunktion $\mathrm{U}_{\mathrm{Env}}(\mathrm{x})$ nach Anhang B, Gleichung (B.30a) von

$$
\mathrm{U}_{\mathrm{Env}}(\mathrm{x}) \approx \frac{\mathrm{C}}{\sqrt{\pi \mathrm{k}_{\mathrm{F}} \mathrm{x}}} \cdot \mathrm{h}_{\mathrm{z}}(\mathrm{x})
$$

Für die weitere Diskussion werden zwei Zugänge benutzt. Der analytische Zugang besteht in einer Kurvendiskussion von Gleichung (8.2) und liefert quantitative Informationen über die Temperaturabhängigkeit der Einhüllendenform der Thermospannung.

Im qualitativen Zugang werden grafisch die Elektronen-Diffusionsströme über die Tunnelbarriere interpretiert (s. auch Kap. 3.2). Diese Art der Beschreibung ermöglicht, die Einhüllende qualitativ zu verstehen. Hierzu sind zwei Abbildungen sehr hilfreich; Abb. 8.11 enthält in (a) einen Ausschnitt der LDOS aus Abb. 8.9, in (b) die Differenz der Fermifunktionen für das Beispiel $\mathrm{T}_{\mathrm{P}}=110 \mathrm{~K}$ und $\Delta \mathrm{T}=1 \mathrm{~K}$ sowie in (c) die nach Gleichung (8.2) berechnete Thermospannung.

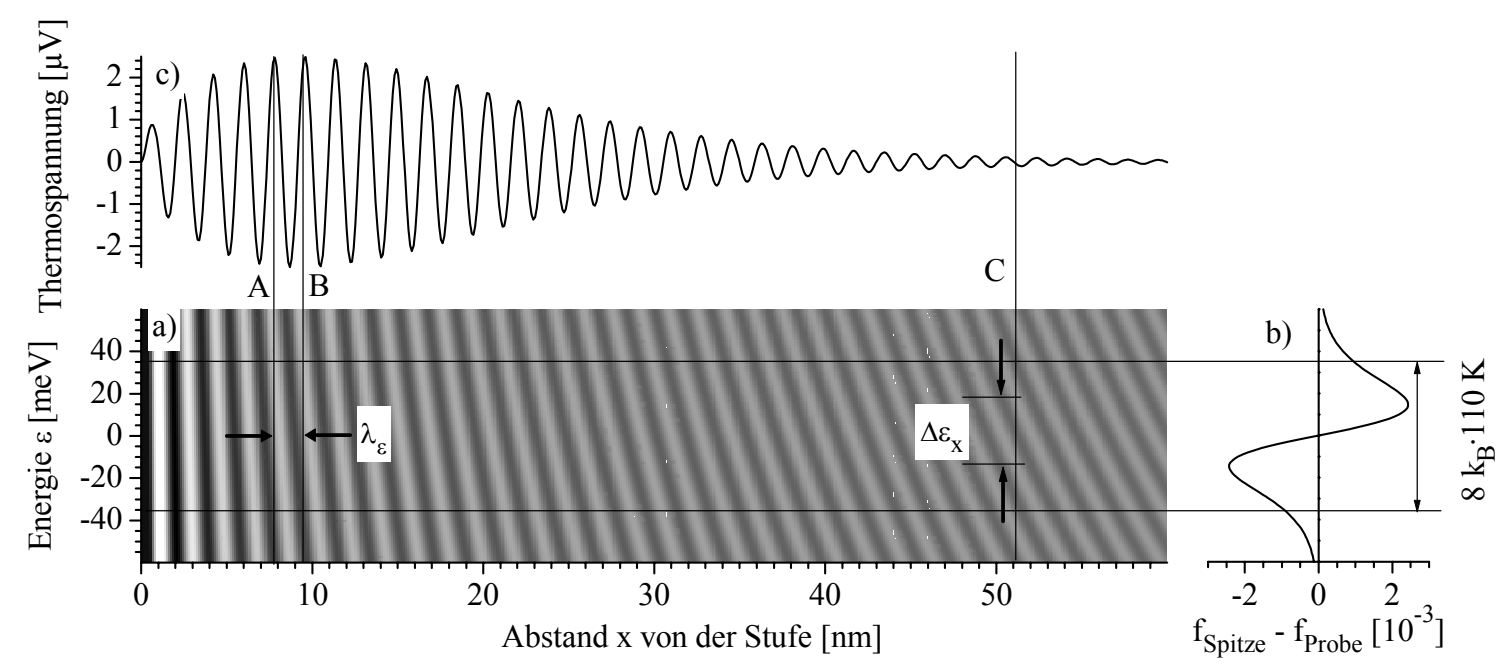

Abb. 8.11 (a) Grauskalendarstellung der LDOS in Abhängigkeit von Energie $\varepsilon$ und Abstand $\mathrm{x}$ von der Stufe; (b) Differenz der Fermifunktionen von Spitze und Probe für $\mathrm{T}_{\mathrm{S}}=111 \mathrm{~K}$ und $\mathrm{T}_{\mathrm{P}}=110 \mathrm{~K}$. (c) zugehörige Thermospannung für $\mathrm{C}=0.2$. 

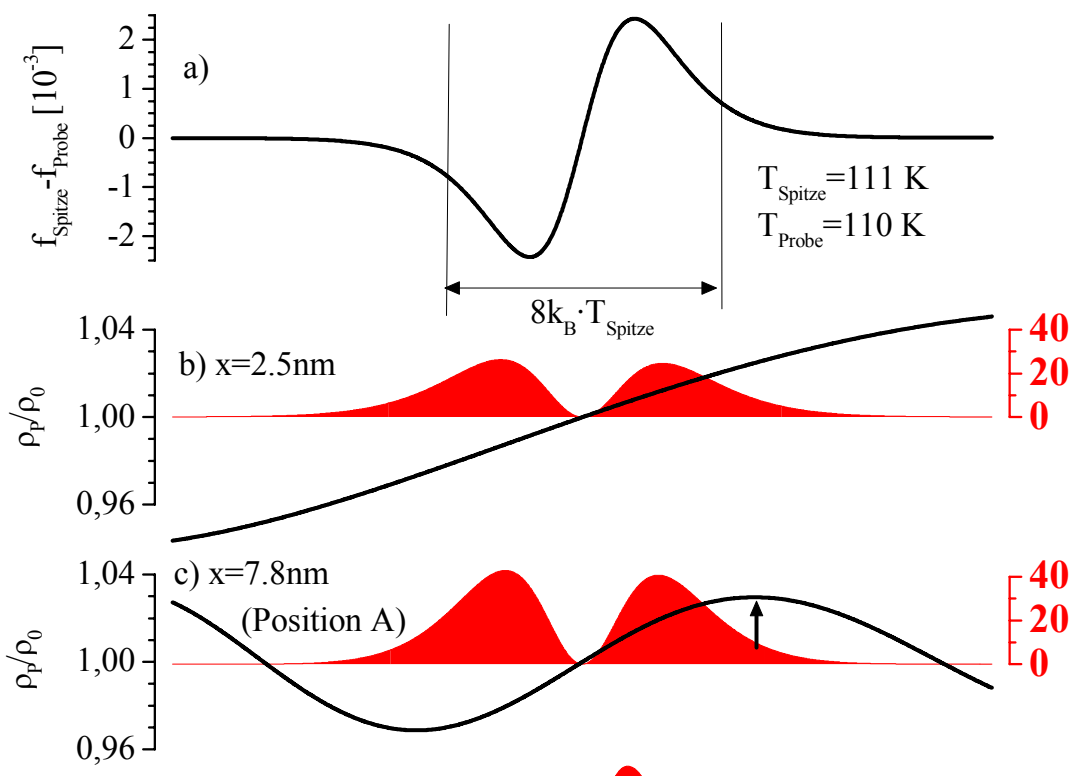

\author{
lokales \\ Maximum in $\mathrm{U}_{\mathrm{Th}}$
}
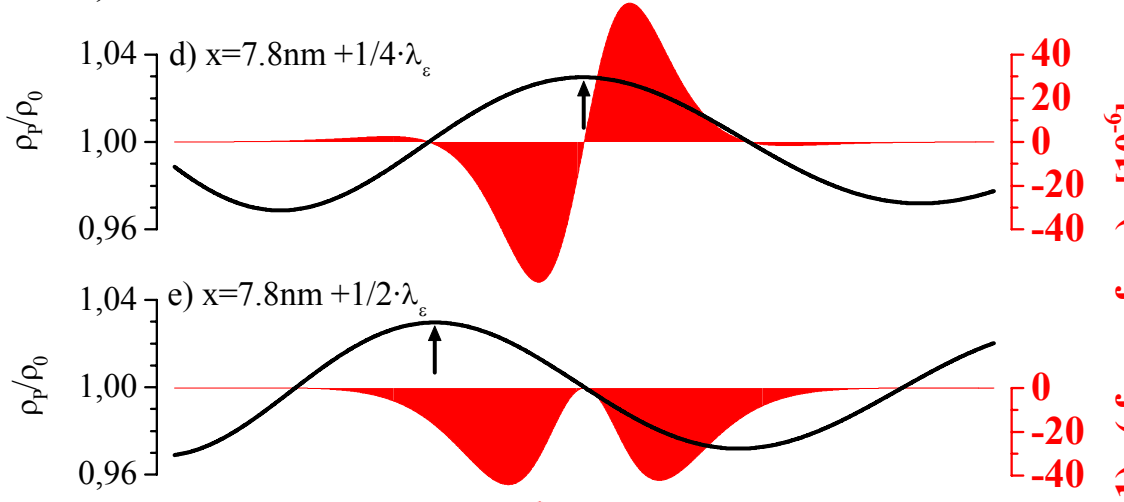

$\stackrel{\mathrm{U}_{\mathrm{Th}}}{=} 0 \mu \mathrm{V}$

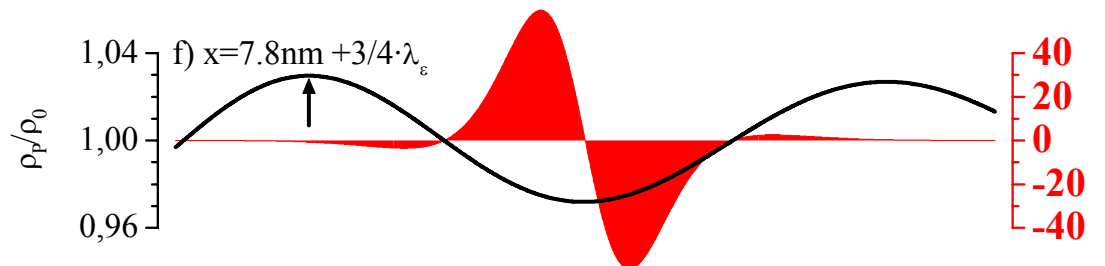

lokales
Maximum in $\mathrm{U}_{\mathrm{Th}}$

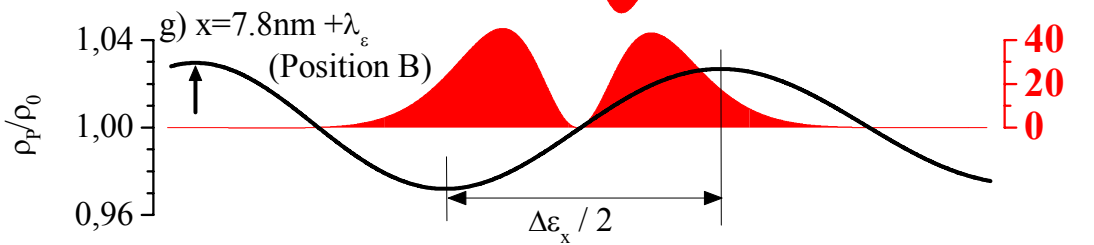

lokales

lokales

Minimum in $\mathrm{U}_{\mathrm{Th}}$

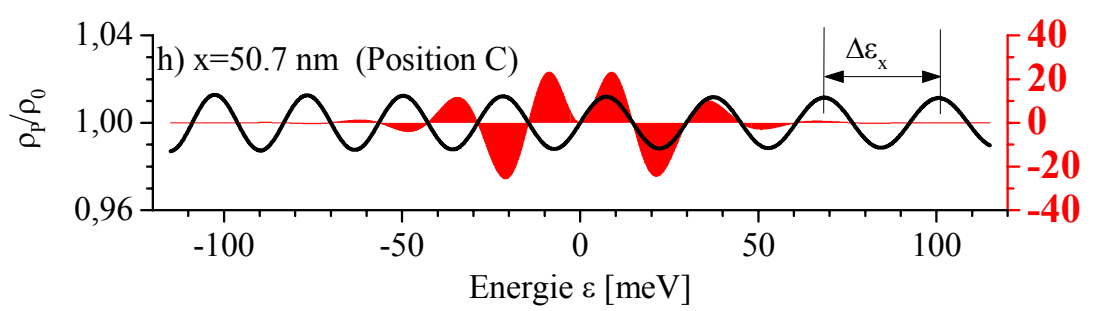

Maximum in $\mathrm{U}_{\mathrm{Th}}$

Abb. 8.12 Einfluss der lokalen Zustandsdichte auf den Tunnelstrom für einige ausgewählte Abstände von der Stufe. (a) Differenz der Fermifunktionen von Spitze und Probe für den Fall $U_{T h}=0$. (b)-(h) Durchgezogene Linie (—): Lokale Zustandsdichte in Abhängigkeit von der Energie, normiert auf die lokale Zustandsdichte einer ungestörten Oberfläche. Flächengraph (—): Zugehöriger Tunnelstrom („Thermostrom“) in Abhängigkeit von der Energie, normiert dargestellt als Abweichung zum Tunnelstrom für eine ungestörte Oberfläche. (c), (g) und (h) sind jeweils als Schnitt A, B und C in Abb. 8.11 markiert. 
In Abb. 8.12 (b)-(h) sind Querschnitte durch die LDOS entlang der Energie-Achse von Abb. 8.11 (a) für verschiedene Abstände von der Stufe dargestellt. Diese werden mit der Differenz der Fermifunktionen von Spitze und Probe multipliziert, um den spektral nach der Energie $\varepsilon$ aufgelösten Tunnelstrom zu erhalten (in Abb. 8.12 als Flächengraph dargestellt). Der resultierende Tunnelstrom entspricht dem Integral über die Energie und ist ein Maß für die lokale Thermospannung

\section{Deutung einer einzelnen Oszillationsperiode}

Abb. 8.12 (c) bis (g) erklärt, warum prinzipiell Oszillationen in der Thermospannung beobachtet werden. Es sind fünf Querschnitte durch die LDOS in Abb. 8.11 (a) entlang der Energie-Achse gezeigt, welche im Ortsraum auf der x-Achse jeweils eine Viertel Wellenlänge $\lambda_{\varepsilon}$ auseinanderliegen. In Abb. 8.11 (a) ist der erste Querschnitt mit „A“ und der letzte mit „, $\mathrm{B}^{\text {“ }}$ gekennzeichnet; beide fallen mit einem Maximum in der Thermospannung zusammen.

Bei der Verschiebung des Querschnittes entlang der x-Achse ist erkennbar, dass sich auch ein Maximum in der LDOS entlang der Energie-Achse verschiebt (markiert mit einem Pfeil in Abb. 8.12. Dementsprechend variiert auch das Spektrum des Tunnelstromes, welches sich als Produkt der lokalen Zustandsdichte mit der Differenz der Fermifunktionen berechnet. Nach jeweils einer halben Wellenlänge ändert sich das Vorzeichen des resultierenden Tunnelstromes, was sich entsprechend in einem Vorzeichenwechsel der Thermospannung äußert.

\subsubsection{Diskussion der Thermospannungs-Einhüllenden}

In diesem Abschnitt sollen folgende Fragestellungen bearbeitet werden:

- Warum besitzt die Thermospannung diese Form der Einhüllenden?

- Welche Temperaturabhängigkeit besitzt die Form der Einhüllenden?

Es bietet sich an, die Form der Einhüllenden in drei Bereiche zu unterteilen. Im ersten, stufennahen Bereich steigt die Amplitude der Thermospannung mit dem Abstand von der Stufe an. Im zweiten Bereich besitzt die Thermospannung eine maximale Amplitude, bevor sie im dritten Bereich für große Abstände von der Stufe ein abklingendes Verhalten zeigt.

Jeder dieser drei Bereiche soll im folgenden qualitativ und quantitativ analysiert werden. Als Übersichtshilfe dient dazu Abb. 8.13, welche neben der analytisch berechneten

\footnotetext{
${ }^{21}$ Dieses Modell entspricht nicht exakt der Messmethode, da in der Abbildung der „Thermostrom“ $\mathrm{I}_{\mathrm{Th}}$ statt der Thermospannung $U_{T h}$ dargestellt ist. Es ist jedoch ein anschauliches Modell, und die Thermospannung für den Fall $\mathrm{I}_{\mathrm{Th}}=0$ ist nahezu proportional zum Thermostrom $\mathrm{I}_{\mathrm{Th}}$ für den Fall $\mathrm{U}_{\mathrm{Th}}=0$.
} 
Thermospannung auch deren Einhüllende sowie die im folgenden vorgestellten Näherungslösungen für den stufennahen bzw. -fernen Bereich darstellt.

Im folgenden sei $\mathrm{T}_{\min }$ die niedrigere und $\mathrm{T}_{\max }$ die höhere Temperatur im System Spitze/Probe.

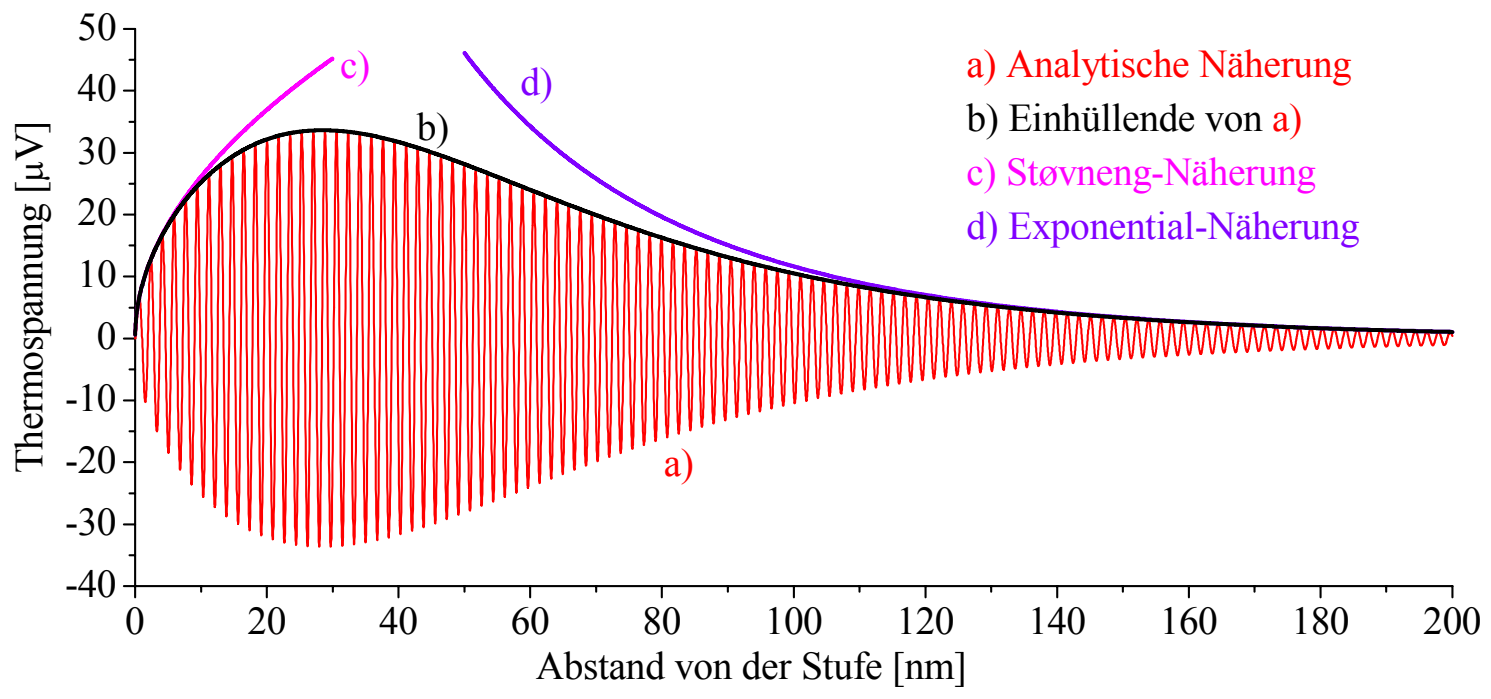

Abb. 8.13 Darstellung der analytisch bestimmten Thermospannung und deren Näherungen für das Beispiel $\mathrm{T}_{\mathrm{P}}=20 \mathrm{~K}, \mathrm{~T}_{\mathrm{S}}=50 \mathrm{~K}$ und $\mathrm{C}=0.2$.

\section{a) Die Thermospannung für kleine Abstände von der Stufe}

Der Anstieg der Thermospannungsamplitude kann anschaulich wie folgt erklärt werden: Im Støvneng-Lipavský-Modell ist die Thermospannung proportional zur Ableitung der LDOS nach der Energie, $d \rho_{\mathrm{P}} /\left.\mathrm{d} \varepsilon\right|_{\varepsilon=0}$, am Orte des elektrochemischen Potenzials $\varepsilon=0$. Grafisch ist diese Größe in Abb. 8.12 als Steigung der LDOS-Kurven bei $\varepsilon=0$ abzulesen. Die Steilheit der LDOS-Oszillationen nimmt mit dem Abstand von der Stufe $z u$, da sich die Abstände der LDOS-Extrema auf der Energieachse schneller verringern, als die Amplitude der LDOS mit dem Abstand abnimmt.

Ein solcher Anstieg der Thermospannungsamplitude ergibt sich auch im analytischen Zugang aus Gleichung (8.2), Die Einhüllende kann nach Anhang B, Gleichung (B.33a), für kleine Abstände von der Stufe genähert werden zu

$$
\mathrm{U}_{\mathrm{Env}}(\mathrm{x}) \approx \frac{\pi^{3 / 2} \cdot \mathrm{k}_{\mathrm{B}}^{2} \cdot \Delta \mathrm{T} \cdot \mathrm{T}_{\mathrm{m}} \cdot \mathrm{C} \cdot}{3 \mathrm{e} \cdot \varepsilon_{\mathrm{F}}} \cdot \sqrt{\mathrm{k}_{\mathrm{F}} \mathrm{x}}
$$

Dasselbe Ergebnis wird bei Anwendung des Støvneng-Lipavský-Formalismus auf die LDOS der Oberflächenzustände erhalten [Eng 1]. Die Thermospannung nahe der Stufe wird also wie folgt beschrieben: 
- Die Einhüllende der Thermospannung steigt wurzelförmig mit dem Abstand x von der Stufe an.

- Die Thermospannung skaliert proportional zur mittleren Temperatur $T_{m}$ von Spitze und Probe sowie zur Temperaturdifferenz $\Delta \mathrm{T}$.

- Für den Spezialfall $T_{\max } » T_{\min }$ skaliert die Thermospannung aufgrund der Identität $\Delta \mathrm{T} \cdot \mathrm{T}_{\mathrm{m}}=1 / 2 \cdot\left(\mathrm{T}_{\mathrm{S}}^{2}-\mathrm{T}_{\mathrm{P}}^{2}\right)$ quadratisch zur höchsten Temperatur $\mathrm{T}_{\max }$ im System Spitze/Probe.

\section{b) Die Thermospannung für große Abstände von der Stufe}

Im Støvneng-Lipavský-Modell hört die Zunahme der Oszillationsamplitude mit dem Abstand erst auf, wenn die LDOS nicht mehr als linear innerhalb der thermischen Verbreiterung angenommen werden kann.

Dieser Fall tritt für große Abstände von der Stufe auf (s. Abb. 8.12 (h)]. Mit zunehmendem Abstand von der Stufe rücken die LDOS-Extrema auf der Energie-Achse immer dichter zusammen, bis auch innerhalb der thermischen Verbreiterung der Fermifunktionen mehrere aufeinanderfolgende LDOS-Extrema $\mathrm{zu}$ finden sind. Multipliziert mit der FermiDifferenzfunktion zeigt das Spektrum der thermischen Diffusionsströme ein mehrfach wechselndes Vorzeichen (s. Abb. 8.12 (h) . Integriert über die Energie führt dies zu einer raschen Abnahme des resultierenden Thermostromes und damit der Oszillations-Amplitude der Thermospannung.

In Amtrang D, Gieichung (B.38) ist gezeigt, dass unter den Bedingungen $x \gg \frac{\varepsilon_{\mathrm{F}}}{\pi \cdot \mathrm{k}_{\mathrm{F}} \cdot \mathrm{k}_{\mathrm{B}}|\Delta \mathrm{T}|}$ und $\mathrm{x} » \frac{\varepsilon_{\mathrm{F}}}{\pi \cdot \mathrm{k}_{\mathrm{F}} \cdot \mathrm{k}_{\mathrm{B}} \mathrm{T}_{\text {min }}}$ die Einhüllende der Thermospannung in Gleichung (8.2) genähert werden kann zu

$$
\mathrm{U}_{\mathrm{Env}}(\mathrm{x})=\frac{2 \mathrm{C}}{\mathrm{e}} \cdot \sqrt{\frac{\pi}{\mathrm{k}_{\mathrm{F}} \mathrm{x}}} \cdot \frac{\mathrm{k}_{\mathrm{B}} \mathrm{T}_{\min }}{\exp \left(\pi \cdot \mathrm{k}_{\mathrm{F}} \mathrm{x} \cdot \mathrm{k}_{\mathrm{B}} \mathrm{T}_{\min } / \varepsilon_{\mathrm{F}}\right)}
$$

Die stehenden Wellen in der Thermospannung klingen also für große Abstände exponentiell ab. An diesem Ergebnis überrascht, dass die höhere Temperatur $T_{\max }$ nicht mit eingeht. Im praktischen Experiment bedeutet dies, dass die räumliche Skala, auf welcher Interferenzphänomene abklingen, wesentlich durch die jeweils tiefste Temperatur $\mathrm{T}_{\min }$ im System Spitze/Probe bestimmt ist. 
Dieses Verhalten kann qualitativ verstanden werden, wenn die Differenz zweier Fermifunktionen für einen großen Temperaturunterschied betrachtet wird, wie in Abb. 8.14 dargestellt.

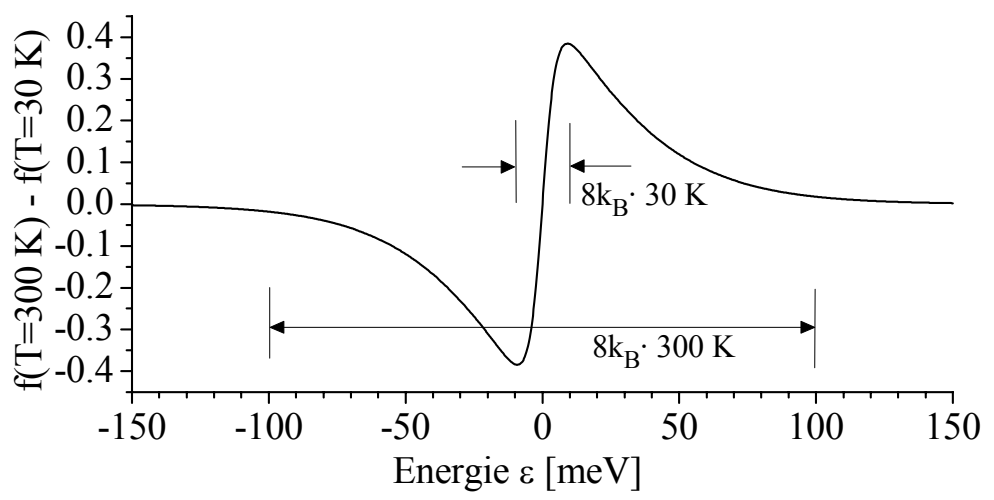

Abb. 8.14 Differenz zweier Fermifunktionen bei $300 \mathrm{~K}$ und $30 \mathrm{~K}$

Die Differenz der Fermifunktionen zeigt um $\varepsilon=0$ einen Bereich mit relativ hoher Steigung zwischen zwei Extrema. Die energetische Breite dieses Bereiches fällt zusammen mit der thermischen Verbreiterung der Fermifunktion mit der kälteren Temperatur (Im Beispiel von Abb. 8.14 sind dies $8 \mathrm{k}_{\mathrm{B}} \cdot 30 \mathrm{~K}=21 \mathrm{meV}$ ). Dieser relativ „steile“ Anstieg liefert für große Abstände von der Stufe den dominanten Term in der Thermospannung und bestimmt deren Abklingverhalten. Dies ist eine wichtige Erkenntnis: Die Thermospannung wird nicht alleine von der Fermifunktion mit der größeren thermischen Verbreiterung bestimmt. Auch die spektral „schärfere“ Fermifunktion für die niedrigere Temperatur liefert einen signifikanten Beitrag.

Dies eröffnet interessante Möglichkeiten: Es reicht aus, entweder die Spitze oder die Probe alleine zu kühlen. Insbesondere ist eine Anordnung denkbar, bei der die Spitze auf möglichst tiefe Temperatur gebracht wird. In der Probe kann unter diesen Umständen bei einer beliebigen Temperatur das Interferenzmuster auch für sehr große Abstände von der Stufe untersucht werden. In bisherigen Untersuchungen wurde das Abklingen stehender Wellen in der Regel durch die (identische) thermische Verbreiterung der Fermifunktionen in Spitze und Probe dominiert [Bür 1, Bür 4]. Diese Problematik hat bisher verhindert, dass bei der Fermienergie eine zuverlässige Kohärenzlänge ${ }^{22}$ für Elektronen bestimmt werden konnte; lediglich untere Grenzwerte konnten bisher angegeben werden [Bür 1, Bür 4]. Im eben genannten Verfahren wäre es hingegen möglich, die lokale Zustandsdichte der Probe unabhängig von deren spektral relativ breiten Fermifunktion mit der spektral „schärferen“ Fermifunktion der kalten Spitze zu spektroskopieren.

\footnotetext{
${ }^{22}$ Die Kohärenzlänge $\mathrm{L}_{\varphi}$ ist die mittlere Wegstrecke, auf der ein Elektron (genauer: Quasiteilchen) durch phasenzerstörende Streuprozesse seine Phaseninformation verliert. Eine weiter Behandlung dieser Thematik ist in Kapitel 8.3 gegeben.
} 


\section{c) Die Thermospannung im Bereich maximaler Oszillationsamplitude}

Es gibt einen charakteristischen Abstand $\mathrm{x}_{\mathrm{m}}$ von der Stufe, bei dem die Oszillationsamplitude maximal wird. An diesem Ort entspricht der energetische Abstand zwischen aufeinanderfolgenden LDOS Minima und Maxima, $\Delta \varepsilon_{\mathrm{x}} / 2$, ungefähr der thermischen Verbreiterung (s. Abb. 8.11 (a) und Abb. 8.12 (g)). Bei $x_{m}$ existiert ein Übergangsbereich, wo das Støvneng-Lipavský-Modell seine Gültigkeit verliert und das exponentielle Abklingen einsetzt.

Dieser Abstand $\mathrm{x}_{\mathrm{m}}$ wird in Anhang B, Gleichung (B.44) abgeschätzt zu

$$
\mathrm{x}_{\mathrm{m}} \approx \frac{\pi \varepsilon_{\mathrm{F}}}{8 \mathrm{k}_{\mathrm{B}} \mathrm{k}_{\mathrm{F}}} \cdot \frac{1}{\mathrm{~T}_{\max }}
$$

Eingesetzt in die Näherungsformel (8.3) nahe der Stufe ergibt sich als oberer Grenzwert $\mathrm{U}_{\mathrm{Th} \text {,max }}$ für die Thermospannungsamplitude:

$$
\mathrm{U}_{\mathrm{Th}, \max }<\frac{\pi^{2} \cdot \mathrm{k}_{\mathrm{B}}^{3 / 2} \cdot \mathrm{C}}{6 \cdot \mathrm{e} \cdot \sqrt{2 \cdot \varepsilon_{\mathrm{F}}}} \cdot \Delta \mathrm{T} \cdot \sqrt{\mathrm{T}_{\max }} .
$$

Hieraus lassen sich die wesentliche Eigenschaften für das Maximum der Oszillationseinhüllenden ablesen:

- Die maximale Oszillationsamplitude ist in einem Abstand $x_{m}$ von der Stufe zu finden, welcher sich wie $1 / \mathrm{T}_{\max }$ zur höchsten Temperatur im System Spitze/Probe verhält. Für tiefe Temperaturen kann somit die Amplitude der Interferenzerscheinungen in der Thermospannung bis auf relativ große Abstände von der Stufe ansteigen.

- Die Amplitude der Thermospannung kann einen Maximalwert proportional zu $\Delta \mathrm{T} \cdot \sqrt{\mathrm{T}_{\max }}$ nicht überschreiten. Trotz des Anstieges in Stufennähe gibt es deshalb auch im Grenzfall $\mathrm{T}_{\max } \rightarrow 0$ keine Divergenz in der Thermospannungsamplitude. 


\section{d) Temperaturabhängigkeit von $U_{T h}(x)$}

Abb. 8.15 bis Abb. 8.17 gibt einen Überblick über die aus Gleichung (8.2a) erhaltenen Thermospannungs-Einhüllenden der stehenden Wellen für verschiedene Temperaturkombinationen.

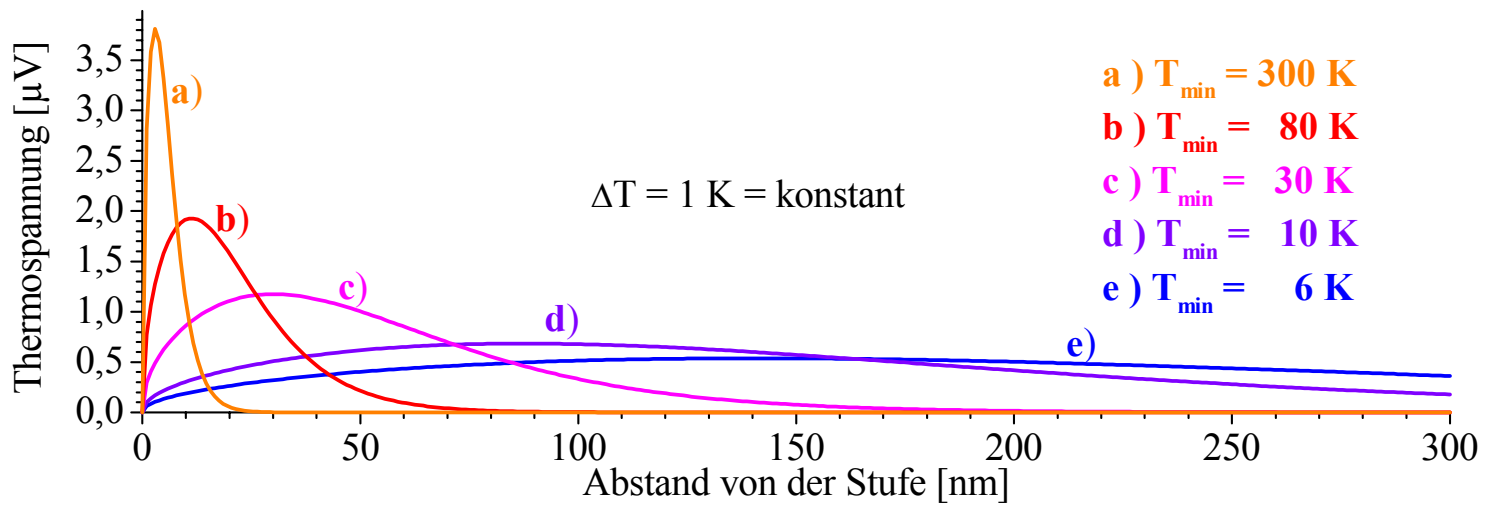

Abb. 8.15 Einhüllende von $\mathrm{U}_{\mathrm{Th}}(\mathrm{x})$ für eine kleine Temperaturdifferenz $\Delta \mathrm{T}=1 \mathrm{~K}$.

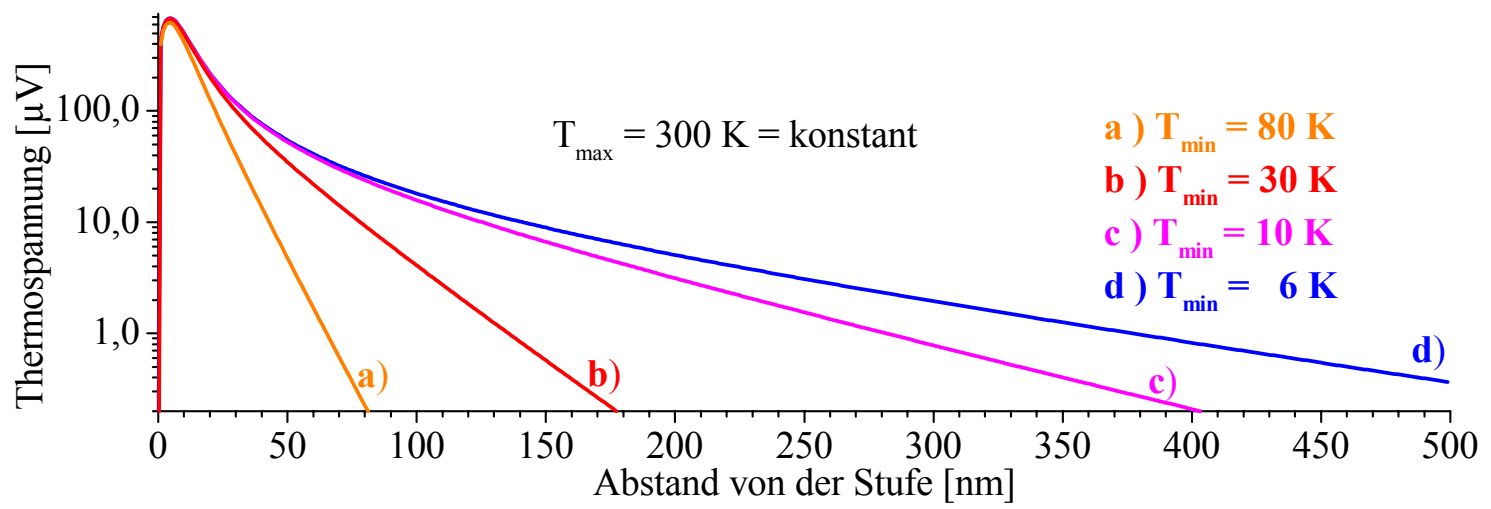

Abb. 8.16 Logarithmische Auftragung der Einhüllenden von $U_{T h}(x)$ für $T_{\max }=300 \mathrm{~K}$.

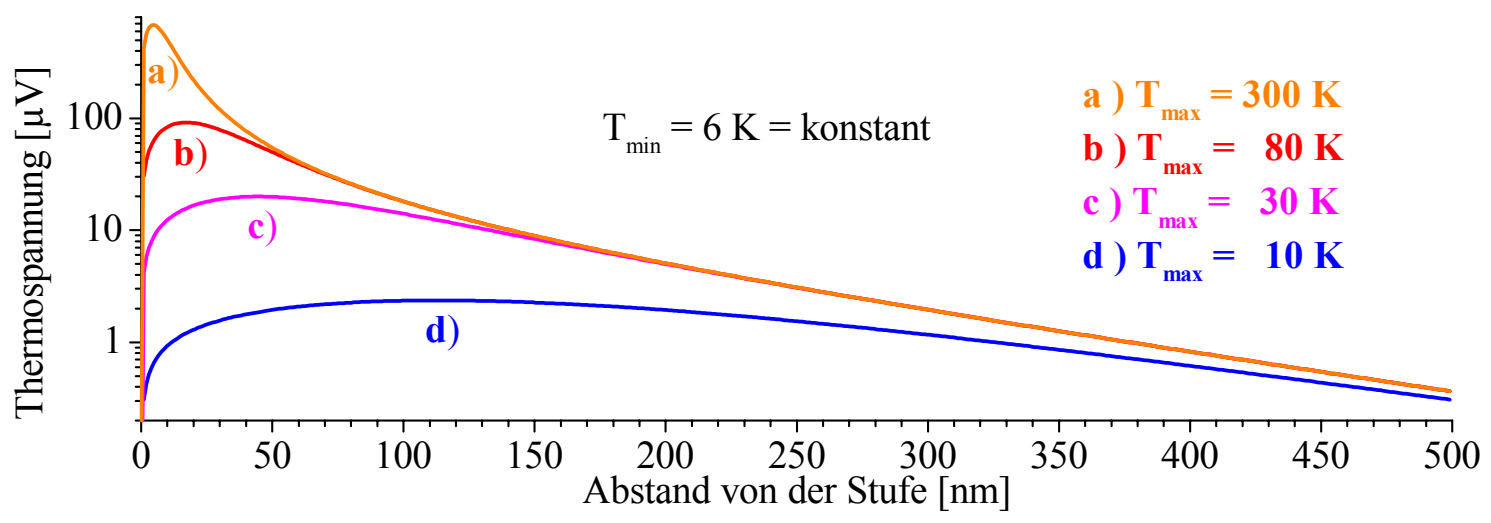

Abb. 8.17 Logarithmische Auftragung der Einhüllenden von $U_{T h}(x)$ für $T_{\min }=6 \mathrm{~K}$. 
Zusammenfassend können bezüglich der Temperaturabhängigkeit folgende Aussagen getroffen werden:

- Die höhere Temperatur $\mathrm{T}_{\max }$ prägt die Form der Thermospannungs-Einhüllenden im stufennahen Bereich. Dies betrifft primär den Abstand $x_{m}$ des Einhüllenden-Maximums von der Stufe $\left(x_{m} \propto 1 / T_{\max }\right.$ nach Gleichung (8.5) s. Abb. 8.15 und Abb. 8.17. Für große Temperaturunterschiede bestimmt $\mathrm{T}_{\max }$ die Skalierung von $\mathrm{U}_{\mathrm{Th}}$ im stufennahen Bereich $\left(\mathrm{U}_{\mathrm{Th}} \propto \mathrm{T}_{\max }^{2}\right.$ nach Gleichung (8.3). s. Abb. 8.16).

- Die tiefere Temperatur $\mathrm{T}_{\min }$ prägt das exponentielle Abklingen und die Skalierung der Thermospannungs-Einhüllenden im stufenfernen Bereich (s. Gleichung (8.4) und Abb. 8.17).

\subsubsection{Vergleich mit numerischer Simulation}

Da für die analytische Lösung einige Näherungsannahmen gemacht werden müssen, ist noch deren Genauigkeit zu prüfen. Dies kann durch eine numerische Berechnung der Thermospannung geschehen. Im Detail unterscheidet sich die numerische Simulation von der analytischen Berechnung wie folgt:

- Statt einer um $\varepsilon=0$ linearisierten Dispersionsrelation wird die korrekte parabolische Dispersionsrelation (6.1) verwendet.

- Für die LDOS der Oberflächenzustände wird statt einer Linearkombination von Sinusund Kosinusfunktion die in (8.1) angegebene Form mit der Besselfunktion $\mathrm{J}_{0}$ verwendet. Der Algorithmus hierzu simuliert das Potenziometrieverfahren, wobei der Tunnelstrom im Tersoff-Hamann Modell (s. Anhang B, Gleichung B.1) berechnet wird.

An jedem simulierten Ort x wird zunächst eine Potenzialdifferenz eingestellt. Die Höhe der virtuellen Spitze wird mit einem numerischen Regelalgorithmus eingestellt, bis der berechnete Tunnelstrom dem im Experiment vorgegebenem Sollwert vom $1 \mathrm{nA}$ entspricht. Im nächsten Schritt wird die Potenzialdifferenz zwischen den Fermifunktionen so eingestellt, dass das Tersoff-Hamann Integral den Wert Null ergibt.

Die Ergebnisse dieser dem Experiment so weit wie möglich nachempfundenen Simulation entsprechen mit hoher Genauigkeit der analytisch erhaltenen Näherung. Im grafischen Plot von Abb. 8.13 ist die simulierte Lösung nicht von der analytischen zu unterscheiden. Die Amplituden von simulierter und analytischer Lösung weichen lediglich um $0.02 \%$ voneinander ab. Die größte Abweichung besteht in einer Phasenverschiebung, welche bei etwa $1 \%$ liegt. 


\subsubsection{Vergleich mit dI/dU-Spektroskopie}

Um den Unterschied zwischen den Interferenzmustern zu verdeutlichen, welche einerseits in der Thermospannung und andererseits mittels dI/dU-Spektroskopie (s. Kap. 2.3.2 gemessen werden, kann mit einer leichten Modifikation die oben vorgestellte Simulation zur numerischen Berechnung des dI/dU-Signals benutzt werden. In der Simulation wird dazu das Potenzial der Spitze um einen Betrag dU verändert und die zugehörige Stromänderung dI berechnet. Für einige ausgewählte Temperaturen sind Thermospannung und dI/dU-Signal zum Vergleich in Abb. 8.18 gegenübergestellt.
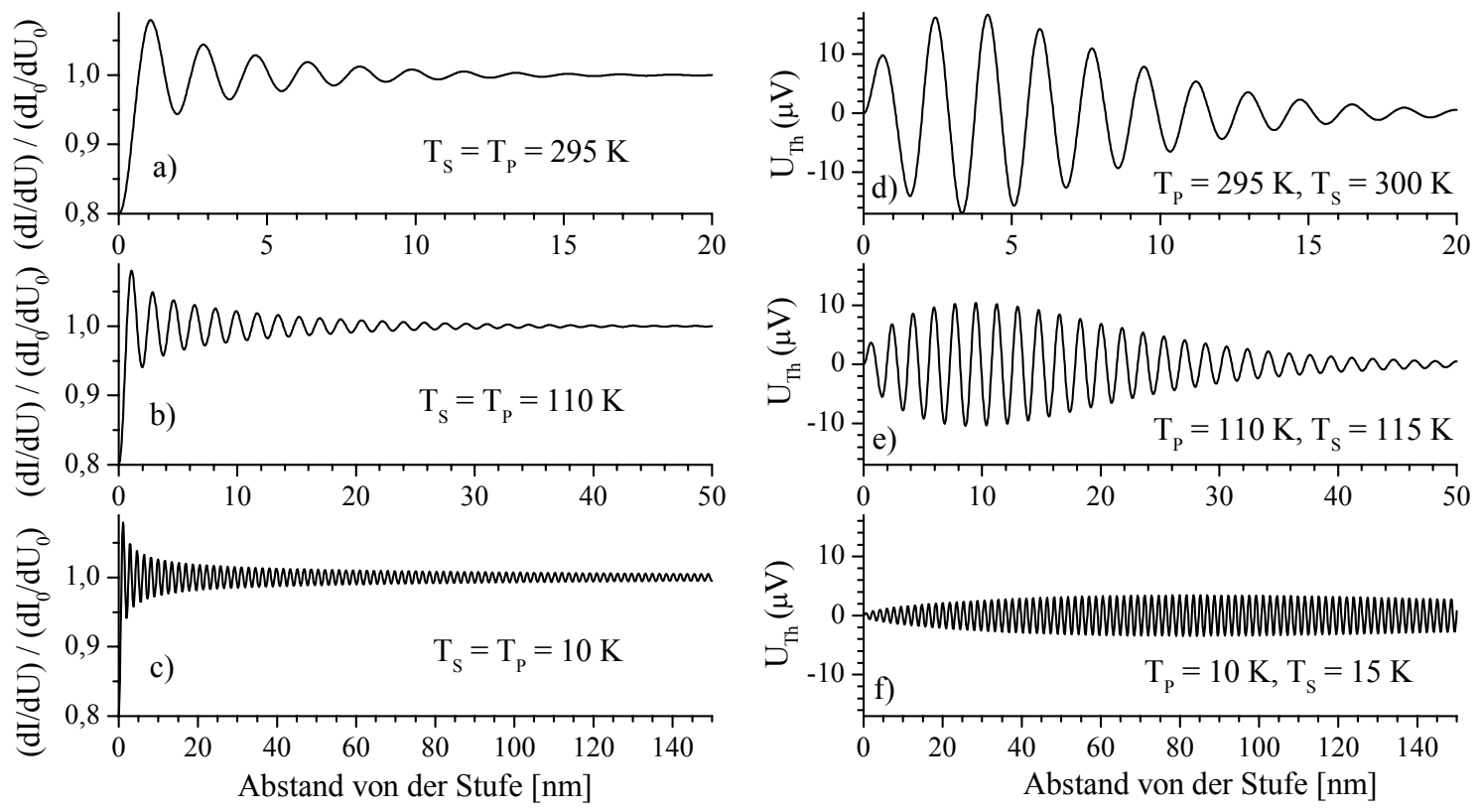

Abb. 8.18 Thermospannung $U_{\mathrm{Th}}(\mathrm{x})$ (rechts) im Vergleich $\mathrm{zu} d \mathrm{~d} / \mathrm{dU}$-Spektroskopie bei $\mathrm{E}_{\mathrm{F}}$ (links, numerisch im Tersoff-Hamann Modell berechnet) für verschiedene Temperaturbereiche.

Im Gegensatz zur Thermospannung klingen die Oszillationen in dI/dU-spektroskopischen Messungen monoton mit dem Abstand ab. Das Maximum der Oszillationen in $\mathrm{U}_{\mathrm{Th}}(\mathrm{x})$ wird bei Raumtemperatur bei $3-5 \mathrm{~nm}$, bei $110 \mathrm{~K}$ bei ca. $10 \mathrm{~nm}$ und bei $10 \mathrm{~K}$ etwa bei $100 \mathrm{~nm}$ Abstand von der Stufe gefunden.

\subsubsection{Einfluss der Spitzen- und der lokalen Volumen- Zustandsdichte}

Alle bisherigen Diskussionen vernachlässigen sowohl die Zustandsdichte der Spitze wie auch die lokale Zustandsdichte der Volumenzustände. Dieser Abschnitt soll die bisher erhaltenen Ergebnisse komplettieren.

Wie Abb. 8.10 entnommen werden kann, ergibt die Berücksichtigung dieser Zustandsdichten einen additiven Term $\mathrm{U}_{\mathrm{Th}+}$ in der Thermospannung: 


$$
\mathrm{U}_{\mathrm{Th}+}(\mathrm{x})=\frac{\left((1-\mathrm{P}) \frac{\rho_{\mathrm{V} 0}^{\prime}}{\rho_{\mathrm{V} 0}}+\frac{\rho_{\mathrm{S} 0}^{\prime}}{\rho_{\mathrm{S} 0}}\right) \cdot \pi^{2} \cdot \mathrm{k}_{\mathrm{B}}^{2} \cdot \Delta \mathrm{T} \cdot \mathrm{T}_{\mathrm{m}}}{3 \mathrm{e} \cdot\left[1+\mathrm{C} \cdot \mathrm{J}_{0}\left(2 \mathrm{k}_{\mathrm{F}} \mathrm{x}\right) \cdot \mathrm{h}_{\mathrm{N}}(\mathrm{x})\right]}=\frac{\mathrm{U}_{\text {Offset }}}{1+\mathrm{C} \cdot \mathrm{J}_{0}\left(2 \mathrm{k}_{\mathrm{F}} \mathrm{x}\right) \cdot \mathrm{h}_{\mathrm{N}}(\mathrm{x})}
$$

Dieser Term ergibt weit weg von der Stufe einen konstanten Offset U Uffset in der Thermospannung. Dieser Offset ist für $\mathrm{x} \rightarrow \infty$ proportional zur Summe aus der differentiellen Zustandsdichte der Spitze, $\frac{\rho_{\mathrm{S} 0}^{\prime}}{\rho_{\mathrm{S} 0}}=\left.\left(\frac{1}{\rho_{\mathrm{S}}} \cdot \frac{\partial \rho_{\mathrm{S}}}{\partial \varepsilon}\right)\right|_{\varepsilon=0}$, und der differentiellen LDOS der Volumenzustände, $\frac{\rho_{\mathrm{V} 0}^{\prime}}{\rho_{\mathrm{V} 0}}=\left.\left(\frac{1}{\rho_{\mathrm{V}}} \cdot \frac{\partial \rho_{\mathrm{V}}}{\partial \varepsilon}\right)\right|_{\varepsilon=0}$, sowie der Temperaturdifferenz $\Delta \mathrm{T}$ und der mittleren Temperatur $\mathrm{T}_{\mathrm{m}}$. In der Nähe einer Stufe wird dieser Offset räumlich durch die Funktion $\left[1+\mathrm{C} \cdot \mathrm{J}_{0}\left(2 \mathrm{k}_{\mathrm{F}} \mathrm{x}\right) \cdot \mathrm{h}_{\mathrm{N}}(\mathrm{x})\right]^{-1}$ moduliert, welche in $\mathrm{Abh} .8 .19$ dargestellt ist. In dieser Funktion bestimmt die Besselfunktion $\mathrm{J}_{0}$ den oszillatorischen Anteil, während $\mathrm{h}_{\mathrm{N}}(\mathrm{x})$ die exponentiell abklingende Einhüllende aufprägt, welche die thermische Verbreiterung der Fermifunktionen berücksichtigt (s. dazu auch Abb. 8.10.
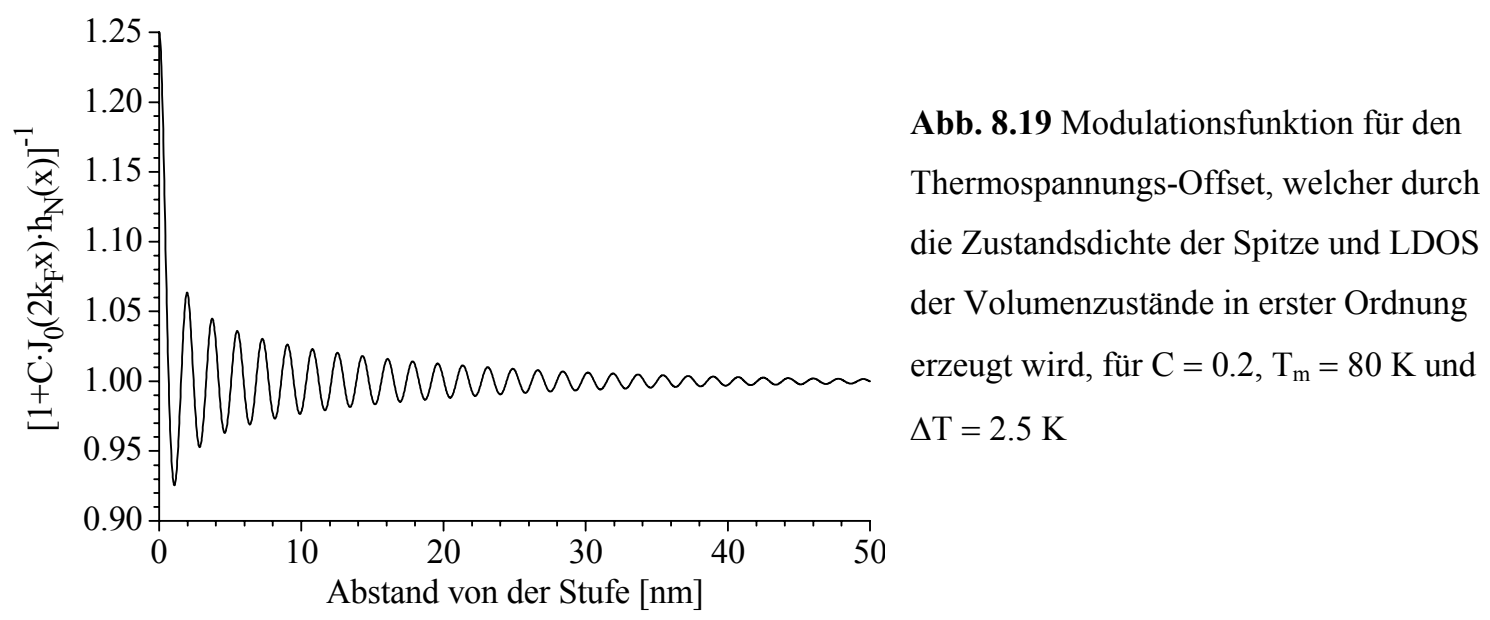

In den vorherigen Abschnitten kann der Einfluss der Nennerfunktion in Gleichung (8.3) vernachlässigt werden, da sich deren Extrema nahe den Nullstellen der Besselfunktion erster Ordnung befinden. Wenn jedoch $\mathrm{U}_{\text {Offset }}$ in der Nähe der Stufe moduliert wird, dann kann dieser die Einhüllende der Thermospannung wesentlich beeinflussen. Es hängt hierbei von der Größenordnung der Offsetspannung $U_{\text {Offset }} a b$, welcher der Terme (8.3) oder (8.7) die Thermospannung in der Nähe der Stufe dominiert.

Zur näheren Analyse wird für den Tunnelkontakt weit weg von der Stufe die Thermokraft $\mathrm{S}_{0}$ berechnet, welche die in (8.7) angegebenen differentiellen Zustandsdichten enthält:

$$
\mathrm{S}_{0}=\frac{\mathrm{U}_{\mathrm{Offset}}}{\Delta \mathrm{T}}=\frac{\pi^{2} \cdot \mathrm{k}_{\mathrm{B}}^{2} \cdot \mathrm{T}_{\mathrm{m}}}{3 \mathrm{e}} \cdot\left((1-\mathrm{P}) \frac{\rho_{\mathrm{V} 0}^{\prime}}{\rho_{\mathrm{V} 0}}+\frac{\rho_{\mathrm{S} 0}^{\prime}}{\rho_{\mathrm{S} 0}}\right)
$$

In Abb. 8.20 ist die Thermospannung $\mathrm{U}_{\mathrm{Th}}(\mathrm{x})$ für verschiedene Thermokräfte $\mathrm{S}_{0}$ aufgetragen: 


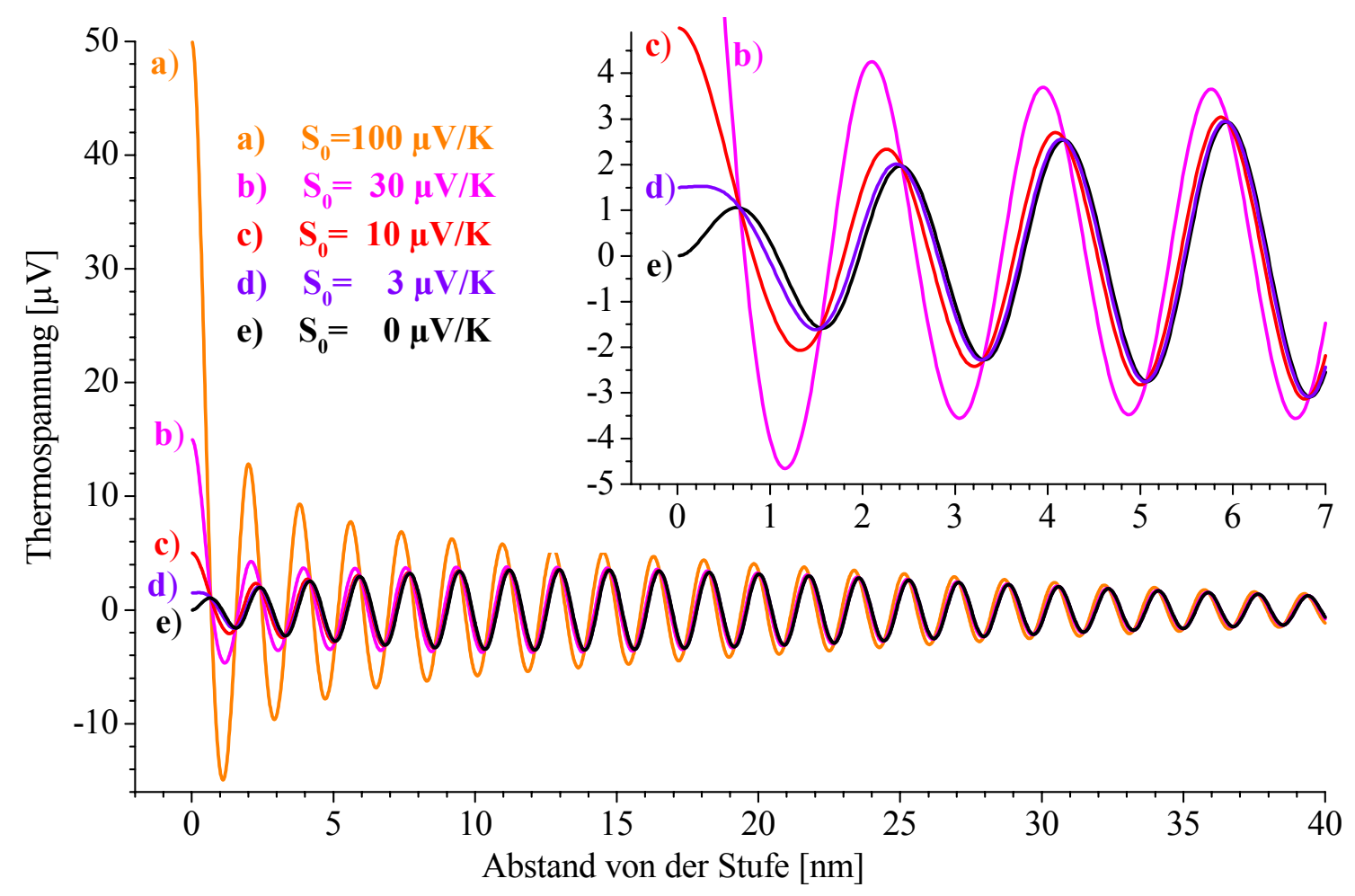

Abb. 8.20 Thermospannung an einer Stufe für verschiedene Thermokräfte $S_{0}$ des Tunnelkontaktes weit weg von der Stufe. Zwecks Vergleichbarkeit wurde der jeweilige Thermospannungs-Offset von jedem Graphen abgezogen. Oben rechts sind die Graphen b) bis e) vergrößert dargestellt.

Der Abb. 8.20 ist $\mathrm{zu}$ entnehmen, dass Einflüsse der Spitzen- oder lokalen Volumenzustandsdichte vernachlässigt werden können, so lange die Thermokraft des Tunnelkontaktes größenordnungsmäßig kleiner als $10 \mu \mathrm{V} / \mathrm{K}$ ist; lediglich die ersten beiden Oszillationszyklen zeigen in diesem Fall signifikante Amplitudenabweichungen. Für größere Thermokräfte nimmt die Form der Thermospannungseinhüllende zunächst in der Nähe der Stufe die einer Besselfunktion nullter Ordnung an, geht aber für weitere Abstände von der Stufe in die bereits in den letzten Abschnitten diskutierte Form über.

Wie in Kap. 4.3.2, Tab. 4.1 dargestellt, treten für den Vakuum-Tunnelkontakt für Au(111) bei mittleren Temperaturen $\mathrm{T}_{\mathrm{m}}$ von $80 \mathrm{~K}-100 \mathrm{~K}$ typischerweise Thermokräfte zwischen $-3 \mu \mathrm{V} / \mathrm{K}$ und $-7 \mu \mathrm{V} / \mathrm{K}$ auf 23 .

In Anbetracht dieser Ergebnisse kann davon ausgegangen werden, dass die Zustandsdichte der Spitze und die LDOS der Probe in den meisten Fällen nur eine vernachlässigbare Modifikation der Einhüllenden der Thermospannung bewirken, welche sich zudem nur auf wenige Oszillationszyklen in der Nähe der Stufenkante beschränken.

\footnotetext{
${ }^{23}$ Es ist zu beachten, dass experimentell bestimmte Thermokräfte eine breite Streuung besitzen, da jede Spitze einen individuellen Anteil zur Thermokraft beiträgt, der sich nach Spitzenmodifikationen auch ändern kann.
} 


\subsection{Bestimmung von Kohärenzlängen aus dem}

\section{Interferenzmuster}

Dieser Abschnitt klärt, in wie weit phasenzerstörende Streuprozesse die Einhüllende des Interferenzmusters beeinflussen. Es wird gezeigt, dass zusätzlich zum thermisch bedingten Fermi-Dirac-Abklingen die Einhüllende auch aufgrund einer endlichen Kohärenzlänge ein exponentielles Abklingen zeigt.

\subsubsection{Dephasierend wirkende Streuprozesse}

Die bisherigen Betrachtungen erfolgen unter der Annahme, dass es keine phasenzerstörenden Streuprozesse gibt. In diesem Bild kann man sich das Tunneln eines Elektrons von der Spitze in die Probe vereinfacht wie folgt vorstellen [Hel 1]: Eine Elektronenwelle transmittiert über die Tunnelbarriere in die Probe und breitet sich dort aus. Bei Anwesenheit einer Streuers, wie z.B. einer monatomaren Stufe, gelangt ein Teil der Welle zur Position der Spitze zurück und interferiert dabei mit sich selbst. Abhängig davon, ob die Welle unterhalb der Spitze konstruktiv oder destruktiv interferiert, erhöht oder erniedrigt sich die „Reflektivität“ der Tunnelbarriere und führt zu den experimentell beobachteten Interferenzmustern.

Während elastische Streuprozesse an Streuern ohne internen Freiheitsgrad ${ }^{24}$ die Phaseninformation erhalten, bewirkt die Streuung von Elektronen mit anderen Elektronen (,e-e“), mit Phononen (,e-ph“), Streuungen an magnetischen Atomen mit Spinflip etc. eine Zerstörung der Phaseninformation [Dat].

Wenn jedoch die Elektronenwelle in der Probe auf dem Weg zwischen Spitze und Stufe aufgrund phasenzerstörender Prozesse ihre Phaseninformation verliert, dann nimmt auch der Grad der Interferenzfähigkeit unterhalb der Spitze mit zunehmendem Abstand vom Streuer ab. Man erwartet demnach ein exponentielles Abklingen des Interferenzmusters auf einer durch die Kohärenzlänge $\mathrm{L}_{\varphi}$ definierten räumlichen Skala [Bür 1, Bür 3-4, Jea].

Für hochangeregte Elektronen (Energien im eV-Bereich oberhalb der Fermienergie) konnte dieser Effekt im Interferenzmuster von $\mathrm{Cu}(111)$ - und $\mathrm{Ag}(111)$-Oberflächen bereits mit hoher Signifikanz nachgewiesen werden [Bür 4].

Ein experimenteller Nachweis für das Abklingen stehender Wellen aufgrund phasenzerstörender Prozesse unterhalb sowie nahe der Fermienergie steht jedoch bisher noch aus. Der Grund hierfür liegt darin, dass die Kohärenzlänge von Elektronen für abnehmende

\footnotetext{
${ }^{24}$ Hierzu zählen alle elastischen Streuprozesse, bei denen kein anderer quantenmechanischer Zustand erzeugt, vernichtet oder geändert wird, also das Elektron in einem kohärenten Eigenzustand verbleibt (strenggenommen kann man aus diesem Grund eigentlich nicht von „Streuung“ sprechen). Zu elastischen Streuern gehören beispielsweise monatomare Stufen, Leerstellen, nicht-magnetische Störatome, Versetzungen, Korngrenzen etc.
} 
Anregungsenergien um Größenordnungen zunimmt (eine quantitative Behandlung wird im nächsten Abschnitt vorgenommen). In mit dem STM gemessenen Interferenzmustern wird bei der Fermienergie das Abklingen der stehenden Wellen hauptsächlich durch die thermische Verbreiterung der Fermifunktionen dominiert [Bür 4].

Es ist deshalb noch eine ungeklärte Frage, ob auch bei Energien weit unterhalb der Fermienergie ein Abklingen im Interferenzmuster gefunden wird. Im eben vorgestellten Modell des Ladungstransportes über die Tunnelbarriere sollte dies der Fall sein, wenn in einer abstrakten Vorstellung ein „Loch“ als positiv geladenes Quasiteilchen von der Spitze in die Probe injiziert wird. Dieses Loch besäße dann für Anregungsenergien unterhalb von $\mathrm{E}_{\mathrm{F}}$ dieselbe Kohärenzlänge wie ein Elektron gleicher Anregungsenergie oberhalb von $\mathrm{E}_{\mathrm{F}}$. Aus dI/dU-spektroskopischen STM-Messungen konnten kürzlich von J. Li und Mitarbeitern signifikante experimentelle Hinweise dafür erhalten werden, dass die Lebensdauer von Lochzuständen in der Probe in tunnelspektroskopischen Messungen wiederzufinden ist [Ber, Kli. Li 5 .

Es sei an dieser Stelle bemerkt, dass das „Tunneln“ von Löchern nur der Versuch einer simplifizierenden Vorstellung des Tunnelprozesses in einem Ein-Teilchenbild ist. Dieser Prozess kann anschaulich im Bilde eines „Quasiteilchens“ erklärt werden: Dieses definieren wir als Elektron/Loch-Paarung, bei der sich Loch und Elektron jeweils auf der gegenüberliegenden Seite der Tunnelbarriere befinden. Den Tunnelprozess verstehen wir als Austauschprozess, bei dem das Elektron über die Tunnelbarriere hinweg den zuvor unbesetzten Zustand des Loches einnimmt und dabei ein Loch in seinem ursprünglichen Zustand hinterlässt. Das oben beschriebene Beispiel, bei dem ein Elektron von der Spitze in die Probe injiziert wird, erweitern wir dahingehend, dass ,gleichzeitig“"während des Tunnelns der Elektronenwelle eine „Lochwelle“ denselben Weg der Elektronenwelle austestet.

Ein Interferenzmuster sollte nur dann detektiert werden können, wenn sowohl das Elektron als auch das Loch keine phasenzerstörenden Streuprozesse erfahren.

Im folgenden werde die Kohärenzlänge $\mathrm{L}_{\varphi}(\varepsilon, T)$ als mittlere freie Weglänge angegeben, welche dieses Quasiteilchen zwischen zwei phasenzerstörenden Streuprozessen zurücklegt ( $\varepsilon$ stehe hier für die Anregungsenergie relativ zum elektrochemischen Potenzial). Mit $\mathrm{L}_{\varphi}$ direkt verknüpft ist die mittlere Lebensdauer $\tau_{\varphi}$ dieses Teilchens. Wenn das Teilchen in Abwesenheit elastischer Streuprozesse (d.h. ballistisch) mit einer Geschwindigkeit v propagiert, dann gilt der Zusammenhang [Dat]

$$
\mathrm{L}_{\varphi}=\mathrm{v} \cdot \tau_{\varphi}
$$

Die Streurate $1 / \tau_{\varphi}$ des Quasiteilchens kann man sich additiv aus der Streuraten $1 / \tau_{\mathrm{e}}$ des Elektrons sowie $1 / \tau_{\mathrm{h}}$ des Loches zusammengesetzt denken (Matthiessensche Regel, [Ash]): 


$$
\frac{1}{\tau_{\varphi}}=\frac{1}{\tau_{\mathrm{e}}}+\frac{1}{\tau_{\mathrm{h}}}
$$

Bei chemisch hochreinen (111)-Edelmetalloberflächen wird die Lebensdauer angeregter Oberflächenzustände hauptsächlich durch die e-e- und e-ph-Streuung dominiert [Bür 4]. Wird diese unabhängig voneinander behandelt, dann kann den Quasiteilchen jeweils eine für den Streuprozess charakteristische Lebensdauer $\tau_{\mathrm{e}-\mathrm{e}}$ und $\tau_{\mathrm{e}-\mathrm{ph}}$ zugeordnet werden.

\subsubsection{Elektron-Elektron-Streuung}

Die Streurate $1 / \tau_{\mathrm{e}}$ von Elektronen im Modell der Fermiflüssigkeit für ein dreidimensionales Gas freier Elektronen berechnet sich zu Noz, Bür 4, McD

$$
\frac{1}{\tau_{\mathrm{e}}}=\frac{1}{\tau_{0}} \cdot \frac{1}{\varepsilon_{\mathrm{F}, \text { Vol }}^{2}} \cdot \frac{\left(\pi \mathrm{k}_{\mathrm{B}} \mathrm{T}\right)^{2}+\varepsilon^{2}}{1+\exp \left(-\varepsilon /\left(\mathrm{k}_{\mathrm{B}} \mathrm{T}\right)\right)}
$$

Hier ist $\varepsilon_{\mathrm{F}, \mathrm{V} \text { l }}$ die Fermienergie des Elektronengases relativ zur Volumen-Leitungsbandunterkante. Die Proportionalitätskonstante $1 / \tau_{0}$ beträgt

$$
\frac{1}{\tau_{0}}=\frac{\pi^{2} \sqrt{3}}{128} \cdot \omega_{\mathrm{SP}},
$$

wobei $\omega_{\text {SP }}$ die Plasmafrequenz des Elektronengases ist. Da die Streuprozesse mit VolumenElektronen an der Oberfläche stattfinden, wird hier für $\omega_{\text {SP }}$ die Oberflächen-Plasmafrequenz verwendet, welche sich nach [Des] $\mathrm{zu} \quad \omega_{\mathrm{SP}}=\omega_{\mathrm{P}} / \sqrt{2}$ aus der Plasmafrequenz $\omega_{\mathrm{P}}$ der Volumenelektronen [Ash] berechnen lässt:

$$
\omega_{\mathrm{SP}}=\frac{\omega_{\mathrm{P}}}{\sqrt{2}}=\sqrt{\frac{\mathrm{ne}^{2}}{2 \varepsilon_{0} \mathrm{~m}_{\mathrm{Vol}}^{*}}} .
$$

Hier ist $\mathrm{n}$ die Dichte der Leitungsbandelektronen mit effektiver Masse $\mathrm{m}_{\mathrm{Vol}}^{*}$, und $\varepsilon_{0}$ ist die Dielektrizitätskonstante. 

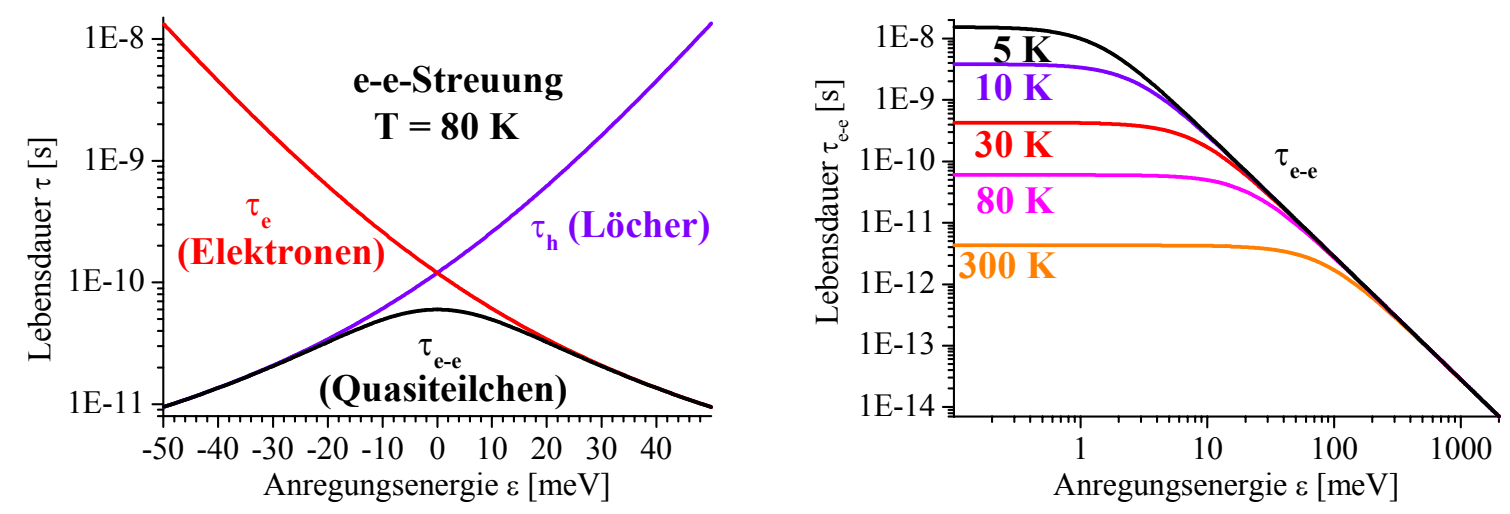

Abb. 8.21 Links: Lebensdauer für angeregte Loch-, Elektronen- und Quasiteilchenzustände unter der Annahme von e-e-Streuung bei $\mathrm{T}=80 \mathrm{~K}$. Rechts: Lebensdauer $\tau_{\mathrm{e}-\mathrm{e}}$ der Quasiteilchenzustände für ausgewählte Temperaturen. Es wurden die Gold-Daten $\mathrm{n}=5.9 \cdot 10^{28} \mathrm{~m}^{-3}, \quad \varepsilon_{\mathrm{F}, \mathrm{Vol}}=5.53 \mathrm{eV}$ [Ash] und $\mathrm{m}^{*}{ }_{\mathrm{Vol}}=1.09 \cdot \mathrm{m}_{\mathrm{e}}$ Len, Mar 3 verwendet.

Für hohe Anregungsenergien $\varepsilon \gg \pi \mathrm{k}_{\mathrm{B}} \mathrm{T}$ zeigt die Lebensdauer eine $\tau_{\mathrm{e}-\mathrm{e}} \propto 1 / \varepsilon^{2}$ Abhängigkeit und ist nahezu unabhängig von $\mathrm{T}$ (Abb. 8.21). Anschaulich wird dies verständlich, wenn man sich den zur Verfügung stehenden Phasenraum unbesetzter Zustände oberhalb von $\mathrm{E}_{\mathrm{F}}$ ansieht, in die das Elektron unter Anregung eines Elektron-Loch-Paares hineinstreuen kann [Ash]. Auf den Systemen $\mathrm{Cu}$ und $\mathrm{Ag}$ konnte für hohe Anregungsenergien im eV-Bereich Lebensdauern für „heiße“ Ladungsträger ermittelt und damit die $\tau_{\mathrm{e}-\mathrm{e}}(\varepsilon) \propto 1 / \varepsilon^{2}$-Abhängigkeit bestätigt werden [Bür 4].

Für Anregungsenergien in der Nähe der Fermienergie, $|\varepsilon|<\pi \mathrm{k}_{\mathrm{B}} \mathrm{T}$, wird die Lebensdauer nur relativ schwach von $\varepsilon$ abhängend von der thermischen Verbreiterung $\mathrm{k}_{\mathrm{B}} \mathrm{T}$ der Fermifunktion bestimmt. Für Energien weit unterhalb von $\mathrm{E}_{\mathrm{F}}$ steigt die Lebensdauer von Elektronenzuständen exponentiell an, da hier auch die Zahl unbesetzter Zustände, in die das Elektron hineinstreuen kann, aufgrund der Fermiverteilung exponentiell abnimmt.

Entsprechende Überlegungen für angeregte Lochzustände führen zu einer zu Gl. (8.11) symmetrischen Streurate $1 / \tau_{\mathrm{h}}$, bei der Lochzustände mit der Anregungsenergie $\varepsilon$ dieselbe Lebensdauer wie Elektronenzustände mit der Anregungsenergie $-\varepsilon$ besitzen:

$$
\frac{1}{\tau_{\mathrm{h}}(\varepsilon)}=\frac{1}{\tau_{\mathrm{e}}(-\varepsilon)}=\frac{1}{\tau_{0}} \cdot \frac{1}{\varepsilon_{\mathrm{F}, \text { Vol }}^{2}} \cdot \frac{\left(\pi \mathrm{k}_{\mathrm{B}} \mathrm{T}\right)^{2}+\varepsilon^{2}}{1+\exp \left(\varepsilon /\left(\mathrm{k}_{\mathrm{B}} \mathrm{T}\right)\right)}
$$

Die Quasiteilchen-Streurate $1 / \tau_{\mathrm{e}-\mathrm{e}}$ ergibt sich schließlich zu

$$
\frac{1}{\tau_{\mathrm{e}-\mathrm{e}}}=\frac{1}{\tau_{\mathrm{e}}}+\frac{1}{\tau_{\mathrm{h}}}=\frac{1}{\tau_{0}} \cdot \frac{1}{\varepsilon_{\mathrm{F}, \mathrm{Vol}}^{2}} \cdot\left[\left(\pi \mathrm{k}_{\mathrm{B}} \mathrm{T}\right)^{2}+\varepsilon^{2}\right] .
$$




\subsubsection{Elektron-Phonon-Streuung}

Die Streurate $1 / \tau_{\mathrm{e}-\mathrm{ph}}$ der Quasiteilchen berechnet sich nach $[\mathrm{McD}]$ in einem Debye-Modell zu

$$
\frac{1}{\tau_{\mathrm{e}-\mathrm{ph}}(\varepsilon)}=2 \pi \cdot \int_{0}^{\omega_{\mathrm{D}}} \lambda_{\mathrm{e}-\mathrm{ph}} \cdot\left(\frac{\omega}{\omega_{\mathrm{D}}}\right)^{2} \cdot[1-\mathrm{f}(\varepsilon-\hbar \omega)+2 \cdot \mathrm{n}(\hbar \omega)+\mathrm{f}(\varepsilon+\hbar \omega)] \cdot \mathrm{d} \omega
$$

wobei $\omega_{\mathrm{D}}$ die Debyefrequenz und $\lambda_{\mathrm{e}-\mathrm{ph}}$ der ,electron-phonon mass enhancement parameter“ ist, welcher für Gold den Wert $0.17 \pm 0.05$ besitzt [Gri, Tab. 11.1] Integriert wird über die Fermi-Verteilungsfunktion $\mathrm{f}$ und die Bose-Verteilungsfunktion n. Mit dem von G. Grimvall [Gri, G1. 4.47] angegebenen Ansatz lassen sich auch die Streuraten für Elektronen und Löcher $\mathrm{zu}$

$$
\frac{1}{\tau_{\mathrm{e}}(\varepsilon)}=2 \pi \cdot \int_{0}^{\omega_{\mathrm{D}}} \lambda_{\mathrm{e}-\mathrm{ph}} \cdot\left(\frac{\omega}{\omega_{\mathrm{D}}}\right)^{2} \cdot[\mathrm{n}(\hbar \omega) \cdot[2-\mathrm{f}(\varepsilon-\hbar \omega)-\mathrm{f}(\varepsilon+\hbar \omega)]+1-\mathrm{f}(\varepsilon-\hbar \omega)] \cdot \mathrm{d} \omega
$$

und

$$
\frac{1}{\tau_{\mathrm{h}}(\varepsilon)}=2 \pi \cdot \int_{0}^{\omega_{\mathrm{p}}} \lambda_{\mathrm{e}-\mathrm{ph}} \cdot\left(\frac{\omega}{\omega_{\mathrm{D}}}\right)^{2} \cdot[\mathrm{n}(\hbar \omega) \cdot[\mathrm{f}(\varepsilon-\hbar \omega)+\mathrm{f}(\varepsilon+\hbar \omega)]+\mathrm{f}(\varepsilon+\hbar \omega)] \cdot \mathrm{d} \omega
$$

berechnen. Die Gleichungen (8.16) - (8.18) lassen sich numerisch integrieren (Abb. 8.23). Für die temperaturabhängige Debyefrequenz werden jeweils experimentell erhaltene Werte für die Debye-Temperatur $\theta_{D}$ aus Ref. [Mar 1-2, Geb. Lyn verwendet (Abb. 8.22), aus welcher sich die Debyfrequenz zu

$$
\hbar \omega_{\mathrm{D}}=\mathrm{k}_{\mathrm{B}} \theta_{\mathrm{D}}
$$

berechnet [Ash]. Für Raumtemperatur wird die maximale Phononenfrequenz experimentell zu $\omega_{\mathrm{D}}=2.95 \cdot 10^{13} \mathrm{~Hz}$ bzw. $\theta_{\mathrm{D}}=225 \mathrm{~K}$ ermittelt [Lyn]

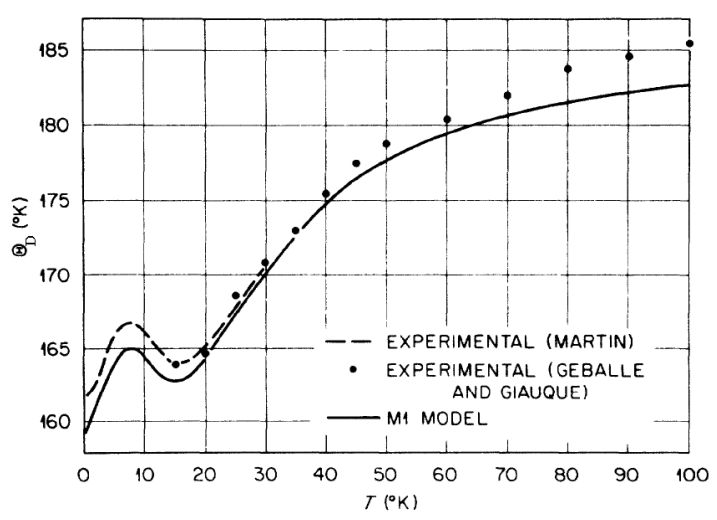

Abb. 8.22 Experimentell erhaltene Werte für die Debye-Temperatur nach D. L. Martin [Mar 1-2] und T. H. Geballe [Geb]. Die durchgezogene Linie stellt eine theoretische Modellierung im ,fourth-neighbor general (M1)“- Modell dar [Lyn] (reproduziert aus [Lyn]. 

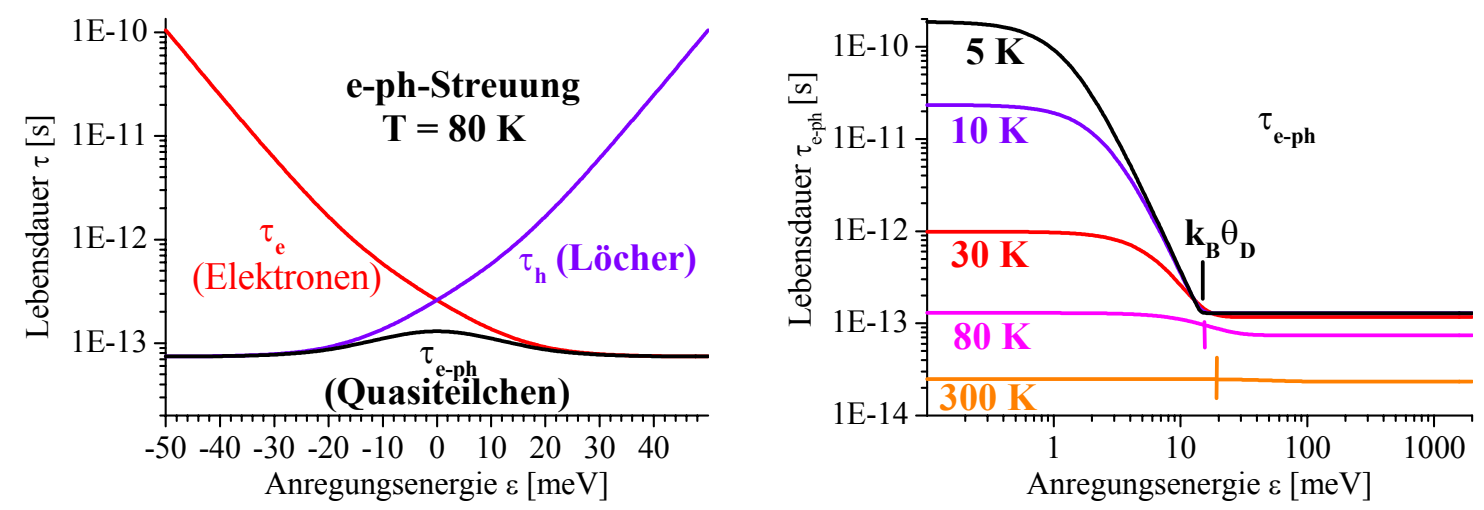

Abb. 8.23 Links: Lebensdauer für angeregte Loch-, Elektronen- und Quasiteilchenzustände unter der Annahme von e-ph-Streuung bei $\mathrm{T}=80 \mathrm{~K}$. Rechts: Lebensdauer $\tau_{\mathrm{e} \text {-ph }}$ der Quasiteilchenzustände für ausgewählte Temperaturen.

Wenn die Quasiteilchen-Anregungsenergien betragsmäßig oberhalb einer Energie $k_{B}\left(\theta_{D}+T\right)$ liegen, dann ist die e-ph-Streurate nahezu konstant, da fast alle möglichen Zielzustände unbesetzt sind ${ }^{25}$ Für kleinere Energien steigt bei e-ph-Streuprozessen die Lebensdauer auf ein Maximum bei $\mathrm{E}_{\mathrm{F}}$ an. Bei Raumtemperatur ist dieses Maximum nur schwach ausgeprägt; die Lebensdauer liegt hier für alle Anregungsenergien zwischen $23 \mathrm{fs}$ und $25 \mathrm{fs}$. Auch bei $80 \mathrm{~K}$ ist die Abhängigkeit nur relativ schwach; sie nimmt von 74 fs bei hohen Anregungsenergien auf 130 fs bei $\mathrm{E}_{\mathrm{F}} \mathrm{Zu}$.

\subsubsection{Abklinglängen in Interferenzmustern}

Ein Vergleich zwischen den Lebensdauern $\tau_{\mathrm{e}-\mathrm{e}}$ und $\tau_{\mathrm{e}-\mathrm{ph}}$ in Abb. 8.24 zeigt, dass bei der Fermienergie die e-ph-Streuung eindeutig dominiert. Die für die e-e-Streuung berechneten Lebensdauern liegen um etwa zwei Größenordnungen über den für e-ph-Streuung berechneten. Erst ab Anregungsenergien von $0.6 \mathrm{eV}$ bei $80 \mathrm{~K}$ und $1 \mathrm{eV}$ bei $300 \mathrm{~K}$ überwiegt die e-e- Streuung. Da jedoch bei der Messung der Thermospannung im wesentlichen nur Zustände innerhalb von $\pm 4 \mathrm{k}_{\mathrm{B}} \mathrm{T}$ um die Fermienergie herum ausgetestet werden, kann für Gold die e-e-Streuung vernachlässigt werden. Wie das rechte Diagramm von Abb. 8.24 zeigt, gilt dies für einen weiten Temperaturbereich von $1 \mathrm{~K}$ bis $1000 \mathrm{~K}$.

\footnotetext{
${ }^{25}$ Man beachte, dass bei der Streuung mit einem Phonon die Energie des Quasiteilchens nur maximal um einen Betrag $\mathrm{k}_{\mathrm{B}} \theta_{\mathrm{D}}$ geändert wird.
} 

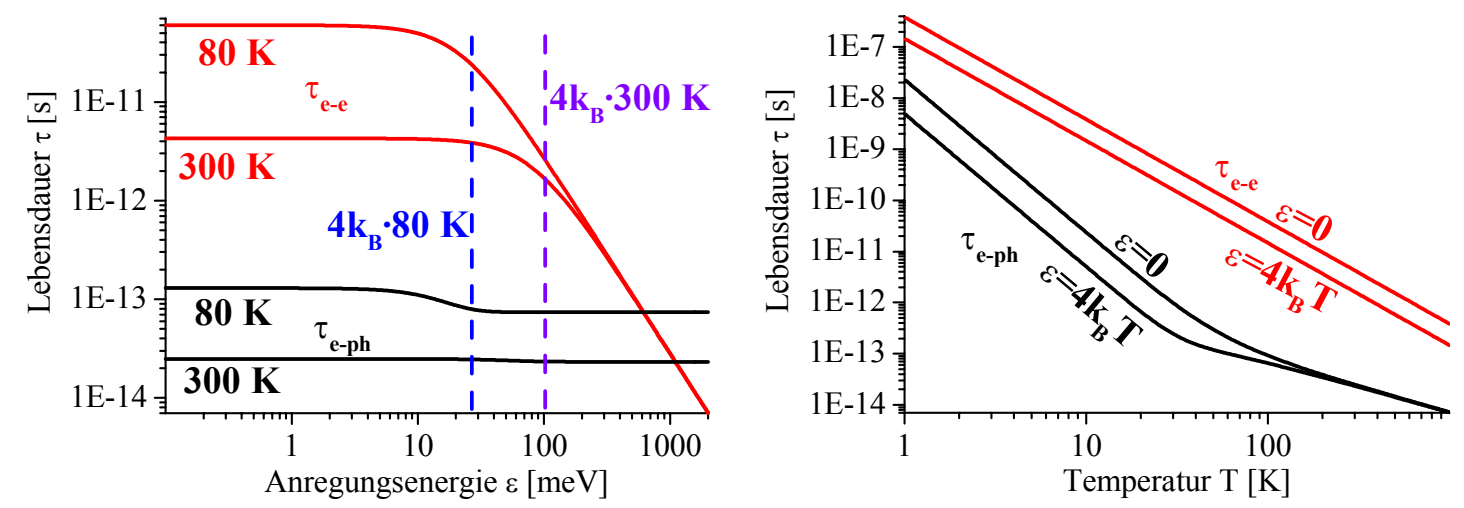

\begin{abstract}
Abb. 8.24 Auftragung der Lebensdauern für Quasiteilchen bei alleiniger e-e-Streuung (-) bzw. e-phStreuung (-). Links: Auftragung über die Anregungsenergie relativ zu $\mathrm{E}_{\mathrm{F}}$ bei $80 \mathrm{~K}$ und $300 \mathrm{~K}$. Die senkrechten gestrichelten Linien geben die „Grenze“ $4 \mathrm{k}_{\mathrm{B}} \mathrm{T}$ der thermischen Verbreiterung der Fermifunktionen an, innerhalb derer der Hauptanteil des Tunnelstromes getragen wird. Rechts: Auftragung über die Temperatur direkt bei der Fermienergie $(\varepsilon=0)$ und bei der „Grenze“ $\varepsilon=4 \mathrm{k}_{\mathrm{B}} \mathrm{T}$ der thermischen Verbreiterung der Fermifunktion.
\end{abstract}

Schätzt man die von der thermischen Fermi-Dirac-Verbreiterung verursachte Abklinglänge $\mathrm{L}_{\mathrm{FD}} \mathrm{ab}$, so erhält man aus Gl. (8.4) die Größenordnung $1 / \mathrm{L}_{\mathrm{FD}}=\pi \cdot \mathrm{k}_{\mathrm{B}} \mathrm{T} \cdot \mathrm{k}_{\mathrm{F}} / \varepsilon_{\mathrm{F}}$. Für $300 \mathrm{~K}$ bzw. $80 \mathrm{~K}$ ergibt sich hieraus $\mathrm{L}_{\mathrm{FD}}(300 \mathrm{~K})=3 \mathrm{~nm}$ bzw. $\mathrm{L}_{\mathrm{FD}}(80 \mathrm{~K})=12 \mathrm{~nm}$. Demgegenüber werden für e-ph-Streuung Abklinglängen von $1 / 2 \cdot L_{\varphi}(300 \mathrm{~K})=9 \ldots 10 \mathrm{~nm} \quad$ bzw. $1 / 2 \cdot \mathrm{L}_{\varphi}(80 \mathrm{~K})=29 \ldots 51 \mathrm{~nm}$ aus Gl. (8.9) und mit den Ergebnissen von Abb. 8.24 abgeschätz ${ }^{26}$. Angesichts dessen ist zu verstehen, dass bei monoton mit dem Abstand von der Stufe abfallenden Topografie- oder dI/dU-spektroskopischen Messungen selbst bei exzellentem Signal/Rausch-Verhältnis eine Bestimmung von $\mathrm{L}_{\varphi}$ schwierig ist. Anders hingegen verhält es sich bei der Messung der Thermospannungseinhüllenden, bei welcher neben dem exponentiellen Fermi-Dirac-Abklingen auch der Abstand von der Stufe im Zählerterm auftritt. Dies führt zu dem schon besprochenen Maximum der Einhüllenden in einem Abstand, bei dem topografische und dI/dU-spektroskopische Messungen bereits um einen Faktor 1/e gedämpft sind. Es besteht also die begründete Vermutung, dass sich Kohärenzlängen mit Hilfe der Thermospannung messen lassen.

Die LDOS an einer Stufenkante, Gl. (8.1), wird bei einer endlichen Kohärenzlänge $\mathrm{L}_{\varphi}$ in guter Näherung wie folgt modifiziert [Bür 4]

$$
\rho_{\mathrm{P}}(\overrightarrow{\mathrm{r}}, \varepsilon)=\rho_{0} \cdot\left(1+\mathrm{C} \cdot \exp \left(\frac{-2 \cdot \mathrm{x}}{\mathrm{L}_{\varphi}(\varepsilon, \mathrm{T})}\right) \cdot \mathrm{J}_{0}(2 \cdot \mathrm{k}(\varepsilon) \cdot \mathrm{x})\right) .
$$

\footnotetext{
${ }^{26}$ Man beachte, dass ein Quasiteilchen den Weg zwischen Spitze und Stufe zweimal zurücklegen muss. Für einen Vergleich mit $\mathrm{L}_{\mathrm{FD}}$ muss $\mathrm{L}_{\varphi}$ deshalb mit einem Faktor $1 / 2$ multipliziert werden.
} 
Die Kohärenzlänge selbst wird mit Gl. (8.9) aus der Geschwindigkeit v(ع) der Elektronen aus den Oberflächenzuständen berechnet:

$$
\mathrm{v}(\varepsilon)=\left.\frac{\mathrm{d} \varepsilon(\mathrm{k})}{\hbar \cdot \mathrm{dk}}\right|_{\mathrm{k}=\mathrm{k}(\varepsilon)}=\frac{\hbar \cdot \mathrm{k}(\varepsilon)}{\mathrm{m}^{*}}=\sqrt{\frac{2 \cdot\left(\varepsilon+\varepsilon_{\mathrm{F}}\right)}{\mathrm{m}^{*}}}
$$

Zusammen mit der e-ph-Lebensdauer $\tau_{\text {e-ph }}$ aus Gl. (8.16) ergibt sich dann

$$
\mathrm{L}_{\varphi}=\sqrt{\frac{2 \cdot\left(\varepsilon+\varepsilon_{\mathrm{F}}\right)}{\mathrm{m}^{*}}} \cdot\left[2 \pi \cdot \int_{0}^{\omega_{\mathrm{D}}} \lambda_{\mathrm{e}-\mathrm{ph}} \cdot\left(\frac{\omega}{\omega_{\mathrm{D}}}\right)^{2} \cdot[1-\mathrm{f}(\varepsilon-\hbar \omega)+2 \cdot \mathrm{n}(\hbar \omega)+\mathrm{f}(\varepsilon+\hbar \omega)] \cdot \mathrm{d} \omega\right]^{-1}
$$

Diese Gleichung wird numerisch integriert und in dem in Kap. 8.2.4 beschriebenen numerischen Berechnungsverfahren für die Thermospannung verwendet. In Abb. 8.25 sind die Kohärenzlängen und in Abb. 8.26 die Thermospannungseinhüllenden für die Temperaturen $\mathrm{T}=80 \mathrm{~K}$ und $\mathrm{T}=295 \mathrm{~K}$ dargestellt.
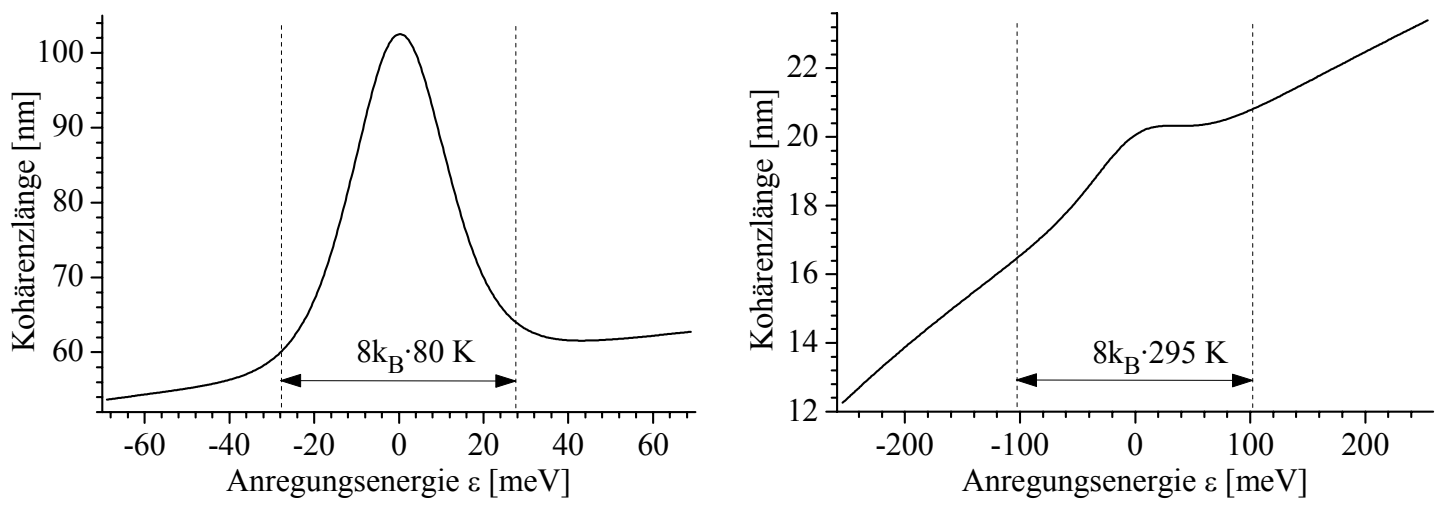

Abb. 8.25 Kohärenzlänge $\mathrm{L}_{\varphi}$ in Abhängigkeit von der Anregungsenergie $\varepsilon$ der Quasiteilchen für die Oberflächenzustände von $\mathrm{Au}(111)$ für die Temperaturen $\mathrm{T}=80 \mathrm{~K}$ (links) und $\mathrm{T}=295 \mathrm{~K}$ (rechts). Man beachte, dass außerhalb der thermischen Verbreiterungszone die Kohärenzlänge mit der Energie zunimmt, obwohl für e-ph-Streuung die Lebensdauer nahezu konstant bleibt, da die Geschwindigkeit der Elektronen zunimmt.
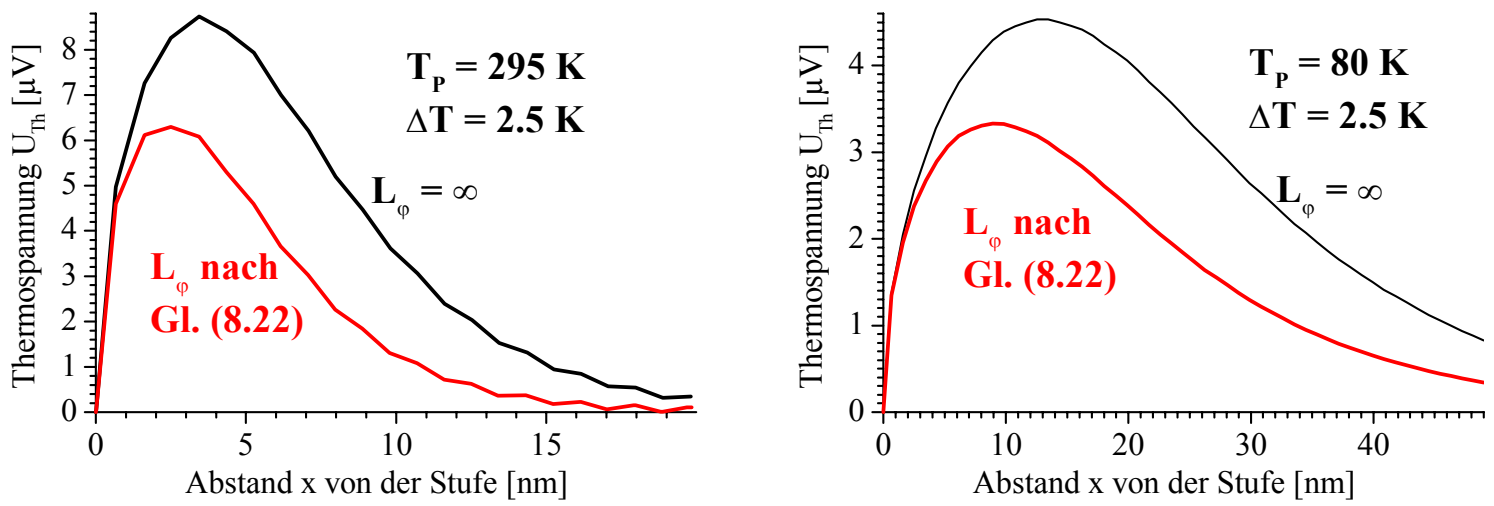

Abb. 8.26 Einhüllende der Thermospannung mit (_) und ohne (_) Berücksichtigung einer nach Gl. (8.22) berechneten Kohärenzlänge für $\mathrm{T}_{\mathrm{P}}=80 \mathrm{~K}$ (links), $\mathrm{T}_{\mathrm{P}}=300 \mathrm{~K}$ (rechts) mit jeweils $\Delta \mathrm{T}=2.5 \mathrm{~K}$. 


\subsection{Quantitative experimentelle Auswertung}

Beim quantitativen Vergleich des Interferenzmusters an einer monatomaren Stufe mit der Theorie sind einige Besonderheiten zu beachten. In der einfachsten Form des Vergleiches werden einzelne Rasterzeilen senkrecht zur Stufe verwendet und die Kurvenform der experimentellen Thermospannung mit der analytischen Rechnung zu vergleichen. Diese Vorgehensweise besitzt jedoch zum einen den Nachteil, dass die Auswahl willkürlich verläuft und damit nicht repräsentativ ist. Zum anderen können auf einzelne Zeilen nicht die statistischen Methoden der Rauschunterdrückung angewendet werden.

Diese Nachteile werden behoben, indem ein Mittelwert der Kurvenform über viele Rasterzeilen gebildet wird. In der Praxis hat sich jedoch gezeigt, dass hier ein neues Problem auftritt: Es ist nicht mehr gewährleistet, dass bei der Mittelung die Extrema übereinander liegen. Im unglücklichsten Fall kann es sogar geschehen, dass aufgrund von Drifterscheinungen, Piezo-Nichtlinearitäten oder leichten Spitzenmodifikationen die Extrema zwar in der Nähe der Stufe bei der Mittelung übereinander liegen, aber nicht mehr in Abständen von 10-20 Wellenzyklen von der Stufe. Die Mittelung ergibt dann für stufenferne Bereiche eine kleinere Amplitude. Abgesehen von dieser Problematik zeigen die Interferenzmuster in der Regel auch eine Überlagerung vom Signal einer geraden Stufe mit einem „Störungsmuster“, welches von vereinzelten Punktdefekten, Kinken oder nicht exakt geradlinigen Stufen herstammt. Diese Effekte in der Summe erlauben eine zuverlässige Bestimmung der Ortsabhängigkeit der Thermospannung bestenfalls für die Mittelung einiger weniger, hochaufgelöster Oszillationszyklen in der Nähe der Stufe.

Für Messungen bei Raumtemperatur ist diese Methode noch genau genug, um die Oszillationen der Thermospannung quantitativ zu analysieren. In Abb. 8.27 werden 60 Zeilen aus der in Abb. 8.1 dargestellten Messung bei $\mathrm{T}_{\mathrm{P}}=295 \mathrm{~K}$ gemittelt und mit der numerisch berechneten Thermospannung für verschiedene Kohärenzlängen verglichen. Für die numerische Berechnung werden zwei Parameter variiert. Die Amplituden-Skalierung ist hauptsächlich von der Temperaturdifferenz $\Delta \mathrm{T}$ zwischen Spitze und Probe abhängig, welche experimentell nur in einem sehr groben Rahmen bekannt ist (s. Kap. 4.3.1) und deshalb hier als Skalierungs-Parameter berücksichtigt wird. Alternativ kann die Amplituden-Skalierung auch durch Variation der ebenfalls nur sehr ungenau bekannten Konstanten C geschehen ${ }^{27}$ Die Ortsabhängigkeit der Thermospannung, d.h. insbesondere deren Abklinglänge, wird durch Vorgabe einer Kohärenzlänge $\mathrm{L}_{\varphi}(\varepsilon=0)$ bei der Fermienergie als zweiten Fit-Parameter

\footnotetext{
${ }^{27}$ Die Konstante $\mathrm{C}$ ist das Produkt aus der Reflektivität $\mathrm{R}$ der Stufe sowie dem Anteil P der LDOS der Oberflächenzustände an der gesamten LDOS in Abwesenheit eines Interferenzmusters. R wird $\mathrm{zu} R=0.4$ angenommen [Sch 1]. P ist abhängig von der individuellen Konfiguration der Spitze und wurde experimentell im Mittel zu $\mathrm{P} \approx 0.5$ bestimmt [Kli, Che 1, Bür 1], so dass in der numerischen Berechnung der Wert $\mathrm{C}=\mathrm{P} \cdot \mathrm{R}=0.2$ verwendet wird.
} 
variiert. Dies geschieht durch eine entsprechende Skalierung der nach Gl. (8.22) berechneten energieabhängigen Kohärenzlänge $\mathrm{L}_{\varphi}$.

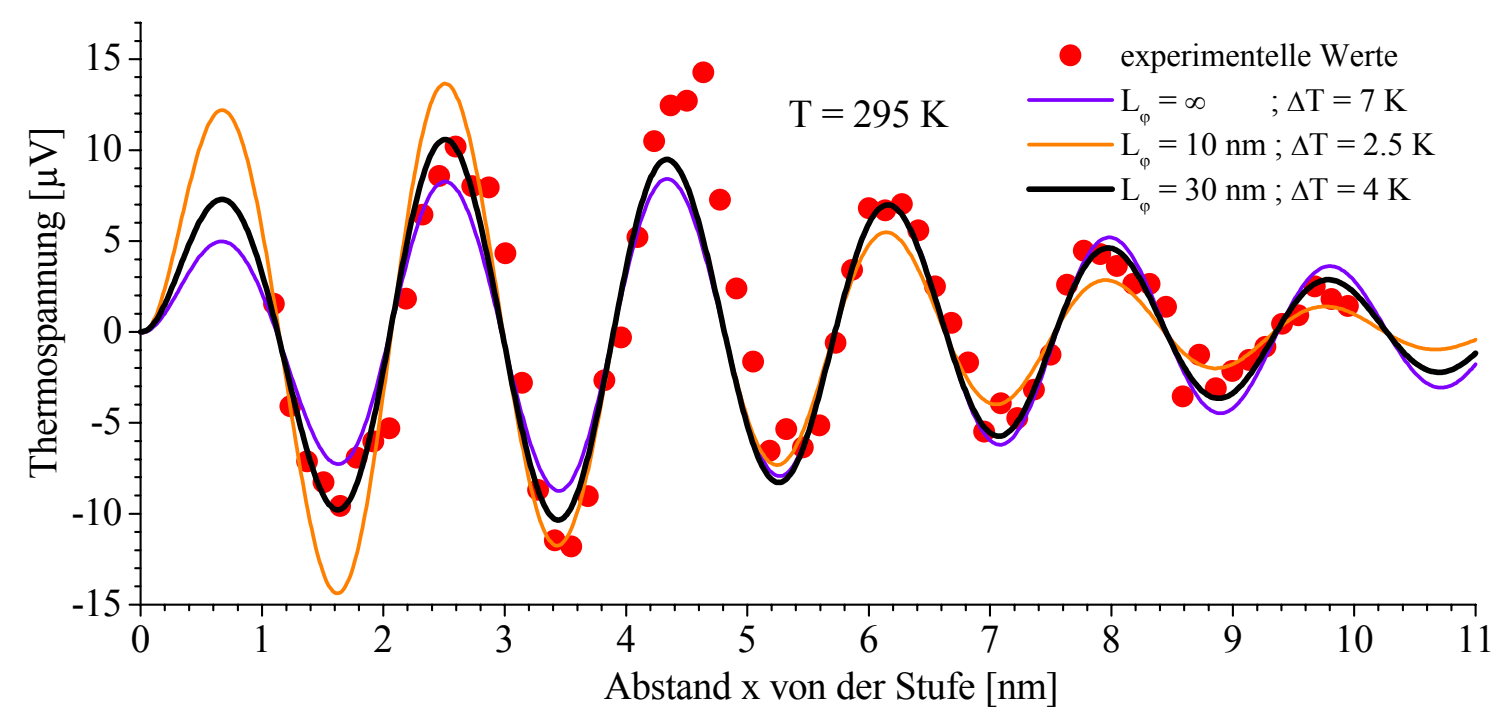

Abb. 8.27 Vergleich der experimentell gemessenen Thermospannung (•) mit den theoretischen Verläufen für $\mathrm{L} \varphi=10 \mathrm{~nm}, \mathrm{~L} \varphi=30 \mathrm{~nm}$ und $\mathrm{L} \varphi=\infty$ bei der Fermienergie $\varepsilon=0$. Die beste Übereinstimmung ergibt sich für $\mathrm{L}_{\varphi}=30 \mathrm{~nm}(-)$.

Eine signifikante Abweichung der theoretischen von der experimentellen Kurvenform ergibt sich für $\mathrm{L}_{\varphi}<10 \mathrm{~nm}$; wie in Abb. 8.27 zu sehen liegt die Amplitude des ersten Oszillationszyklus der theoretischen Kurve deutlich über der experimentell gemessenen, während die letzten drei Zyklen der theoretischen Kurve deutlich stärker abklingen als experimentell gemessen.

Damit ist eine neue untere Grenze von $L_{\varphi}>10 \mathrm{~nm}$ für die Kohärenzlänge bzw. $\tau_{\varphi}=13 \mathrm{fs}$ für die Lebensdauer der Oberflächenzustände von Gold bei der Fermienergie für $T=295 \mathrm{~K}$ gefunden.

Die höchste bisher von Y. Hasegawa und P. Avouris gefundene Kohärenzlänge von $\mathrm{L}_{\varphi}>5 \mathrm{~nm}$ ist von der Fermi-Dirac Verbreiterung dominiert Has 1]. Eine obere Grenze für die Kohärenzlänge kann aus den experimentellen Daten nicht eindeutig bestimmt werden; wie in Abb. 8.27 dargestellt kann auch für $\mathrm{L}_{\varphi}=\infty$ eine innerhalb der experimentellen Unsicherheit liegende Kurve an die Daten angefittet werden, obwohl tendenziell die theoretische Kurve eine zu geringe Amplitude nahe der Stufe und eine zu große Amplitude weit entfernt von der Stufe zeigt. Als ,best guess“ ergibt sich eine Kohärenzlänge von $\mathrm{L}_{\varphi}=30 \mathrm{~nm}$.

Für $\mathrm{LN}_{2}$-gekühlte Gold-Proben wird zur Auswertung der Thermospannungseinhüllenden eine andere Methode angewendet, welche die Problematik der Faltung von Oszillationen während der Mittelung umgeht: Es wird die Oszillationseinhüllenden vor der Mittelung der Rasterzeilen gebildet. Diese Methode besitzt zwar den Nachteil, dass nicht mehr einzelne Oszillationen aufgelöst werden, also die Phasenlage der Oszillationen verloren geht, aber den 
Vorteil, dass ein statistisches Mittel über die ortsabhängige Amplitudenstärke der Thermospannung gebildet werden kann.

In der praktischen Durchführung dieser Methode wird eine Messung zunächst Fouriertiefpassgefiltert, um hochfrequentes Rauschen auf den Oszillationen zu glätten (die Grenzfrequenz wird hierbei hoch genug gewählt, so dass die eigentlich interessierende Form der Einhüllenden nicht beeinflusst wird). Anschließend wird die Einhüllende einer Rasterzeile bestimmt, indem jeweils der halbe Differenzbetrag zwischen zwei aufeinanderfolgenden Extrema bestimmt wird. Die so bestimmten Einhüllenden werden dann gemittelt.

Bei der Interpretation der Einhüllenden $U_{\text {env }}(x)$ muss berücksichtigt werden, dass die Thermospannung ein Hintergrund-Rauschen $U_{R}$ besitzt, welches sich dem Interferenzmuster mit einer Amplitude $\mathrm{U}_{\mathrm{I}}(\mathrm{x})$ überlagert. Dieses „Rauschen“ setzt sich primär aus Interferenzanteilen der Thermospannung zusammen, welche vermutlich von Punktdefekten und Oberflächen-Adsorbaten herstammen und ein p-p Amplitude von ca. $2 \mu \mathrm{V}$ besitzen. Die Einhüllende besitzt die Form

$$
\mathrm{U}_{\text {env }}(\mathrm{x})=\sqrt{\mathrm{U}_{\mathrm{I}}(\mathrm{x})+\mathrm{U}_{\mathrm{R}}}
$$

und kann mit der numerisch berechneten Einhüllenden durch Anpassen der Parameter verglichen werden. $U_{R}$ bestimmt hier den Offset für den stufenfernen Bereich. Als weitere Parameter stehen die Probentemperatur $T_{P}$, die Temperaturdifferenz $\Delta \mathrm{T}$ und die Kohärenzlänge $\mathrm{L}_{\varphi}$ zur Verfügung.

Die experimentelle Bestimmung der Proben- und Spitzentemperatur bei $\mathrm{LN}_{2}$-Kühlung ist mit einem großen Fehler behaftet, so dass diese nur in ihrer Größenordnung bekannt sind und deshalb als Fit-Parameter verwendet wurden. Die Probentemperatur $T_{P}$ wird im Bereich zwischen $80 \mathrm{~K}$ und $130 \mathrm{~K}$ abgeschätz ${ }^{28}$ Die Temperaturdifferenz zwischen Spitze und Probe beträgt in der Größenordnung bis zu 5 Kelvin. Auch hier bewirkt eine Variation von $\Delta \mathrm{T}$ eine Skalierung der Einhüllendenfunktion, während die qualitative Hüllenform (insbesondere die Abklinglänge) hauptsächlich vom Parameter $\mathrm{T}_{\mathrm{P}}$ und der Kohärenzlänge $\mathrm{L}_{\varphi}$ bestimmt wird.

\footnotetext{
${ }^{28}$ Die Sockeltemperatur des STM entspricht mit $79 \mathrm{~K}$ (gemessen von einer thermisch sehr gut angekoppelten Siliziumdiode) relativ gut der Temperatur des flüssigen Stickstoffs als Kühlmittel. Aufgrund von thermischer Rest-Strahlung durch die transparenten Fenster und der nicht perfekten thermischen Ankopplung der Probe an den Sockel ist jedoch damit zu rechnen, dass die Probe deutlich wärmer sein kann.
} 
Das Verfahren der Einhüllenden-Mittelung wird auf die in Abb. 8.28 vorgestellte Messung angewendet. Die Topografie zeigt links und am rechten Rand der Abbildung jeweils monatomare Stufen, welche eine Terrasse der Breite von $90 \mathrm{~nm}$ einschließen. An der linken Stufe ist in der Thermospannung deutlich ein Interferenzmuster zu erkennen. Dieses weist bis zu einer Entfernung von ca. $30 \mathrm{~nm}$ von der Stufe stehende Wellen auf, welche parallel zur Stufe verlaufen.
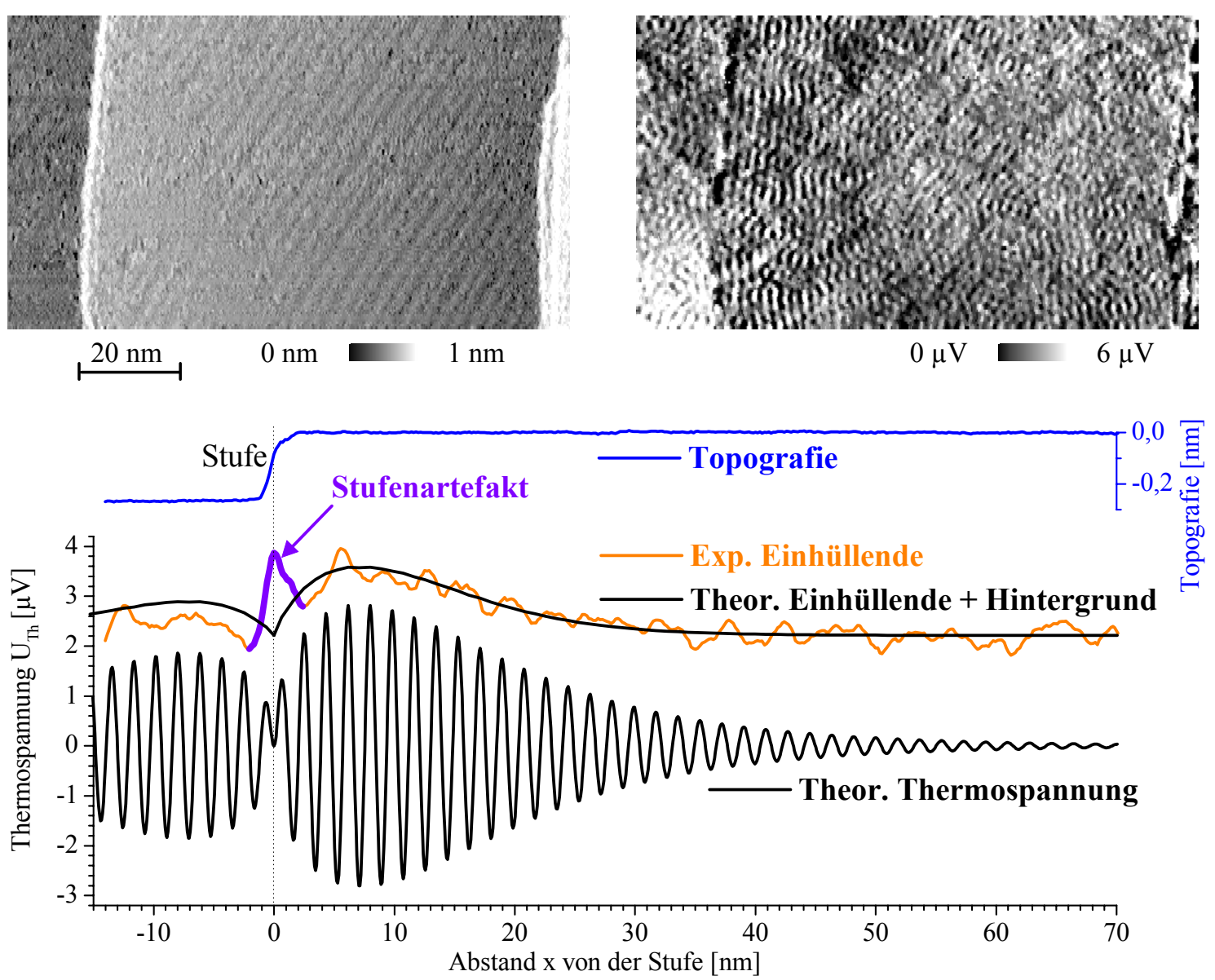

Abb. 8.28 Oben: Topografie (links) und simultan aufgenommene Thermospannung (rechts) auf einer etwa $90 \mathrm{~nm}$ breiten Terrasse. Unten: Experimentell bestimmte Einhüllende der Thermospannung (_) im Vergleich zur theoretischen Thermospannung (-), welche aus der numerisch berechneten Thermospannung für $\mathrm{T}_{\mathrm{P}}=80 \mathrm{~K}, \Delta \mathrm{T}=2.5 \mathrm{~K}$ und $\mathrm{L}_{\varphi}=60 \mathrm{~nm}$ bei der Fermienergie gewonnen wird. Die theoretische Einhüllende wird auf eine Hintergrund-Rauschamplitude von $2.2 \mu \mathrm{V}$ addiert. Die experimentelle Kurve besitzt direkt an der Stufenkante bei $\mathrm{x}=0$ einen Peak ( $($ ), der von der besonderen elektronischen Struktur der Stufe herstammt [Sch 1] und für das Anpassen der numerischen Ergebnisse nicht berücksichtigt wird. 


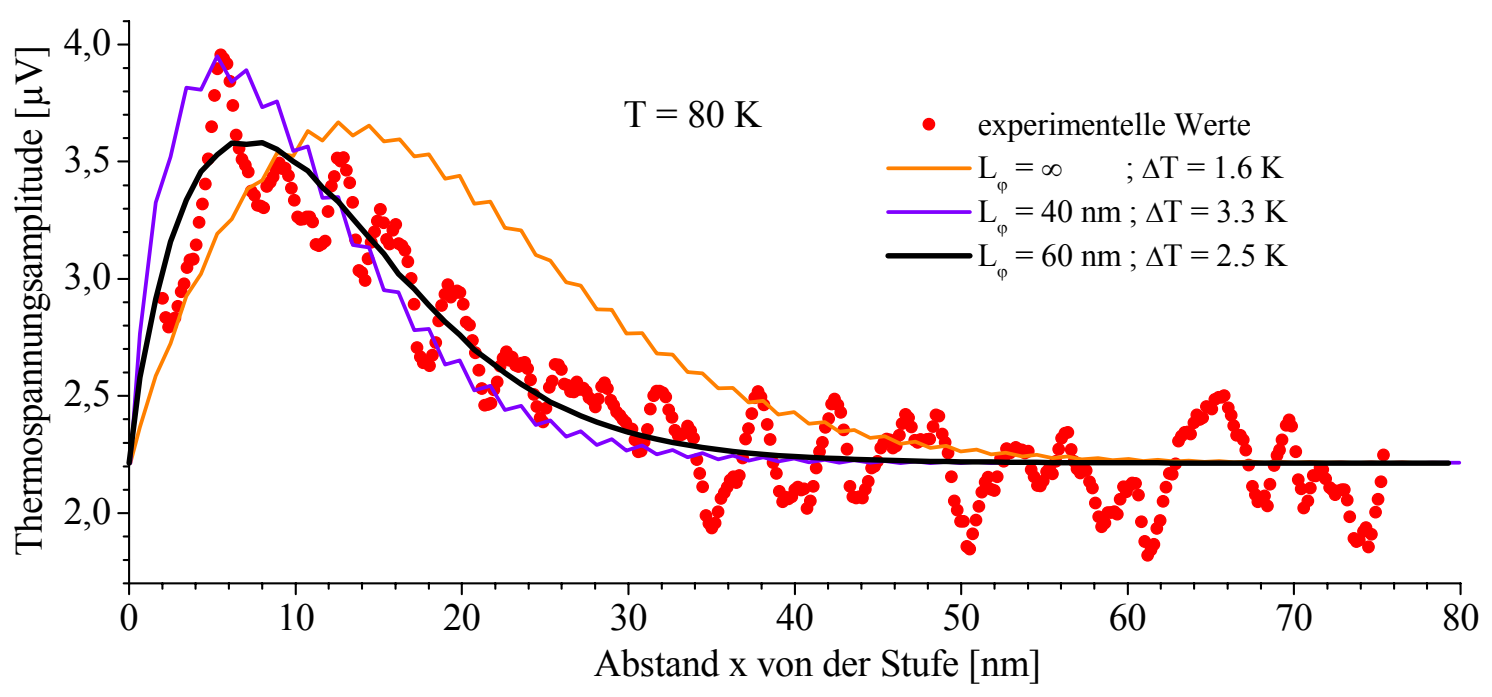

Abb. 8.29 Vergleich der experimentell gemessenen Thermospannungseinhüllenden (•) mit den theoretischen Verläufen für verschiedene angenommene Kohärenzlängen L $\varphi$ bei der Fermienergie $\varepsilon=0$. Die beste Übereinstimmung ergibt sich für $\mathrm{L}_{\varphi}=60 \mathrm{~nm}(-)$. Alle theoretischen Kurven werden auf eine Hintergrund-Rauschamplitude von $\mathrm{U}_{\mathrm{R}}=2.2 \mu \mathrm{V}$ addiert.

Der Vergleich mit der analytisch erhaltenen Einhüllenden ergibt für die Fit-Parameter $\mathrm{T}_{\mathrm{P}}=80 \mathrm{~K}, \Delta \mathrm{T}=2.5 \mathrm{~K}, \mathrm{U}_{\mathrm{R}}=2.2 \mu \mathrm{V}$ und $\mathrm{L}_{\varphi}=60 \mathrm{~nm}$ die beste Übereinstimmung. Eine signifikante Abweichung der theoretischen von der experimentellen Kurvenform ergibt sich für $\mathrm{L}_{\varphi}<40 \mathrm{~nm}$; wie in Abb. 8.29 zu sehen ist. Im Vergleich $\mathrm{zu}$ der experimentellen Einhüllenden steigt in diesen Fall die theoretische Einhüllende zunächst stärker an und klingt mit dem Abstand auch stärker ab. Für $\mathrm{L}_{\varphi}=\infty$ zeigt die theoretische Kurve ebenfalls eine deutliche Abweichung $\mathrm{zu}$ den experimentell gemessenen Werten. Allerdings ist hier $\mathrm{zu}$ beachten, dass die Probentemperatur auch oberhalb von $80 \mathrm{~K}$ liegen kann, so dass das im Experiment beobachtete Abklingen der Thermospannung in diesem Fall durch die FermiDirac-Verbreiterung verursacht werden könnte. Wenn eine Probentemperatur von $T_{P}=110 \mathrm{~K}$ angenommen wird, kann die Thermospannungseinhüllende auch bei einer Annahme von $\mathrm{L}_{\varphi}=\infty$ mit hinreichender Genauigkeit angepasst werden [Eng 1] Aufgrund der unsicheren Temperaturangabe sind somit die in Abb. 8.29 bestimmten Kohärenzlängen als untere Grenzwerte zu verstehen. Da die experimentell gemessene Kohärenzlänge bei $\mathrm{E}_{\mathrm{F}}$ nicht kleiner als $40 \mathrm{~nm}$ sein kann, die Probentemperatur nicht kleiner als $80 \mathrm{~K}$ sein kann und die Kohärenzlänge mit abnehmender Temperatur ansteigt, folgt hieraus:

Bei $80 \mathrm{~K}$ gibt es eine untere Grenze von $L_{\varphi}>40 \mathrm{~nm}$ für die Kohärenzlänge bzw. $\tau_{\varphi}>50$ fs für die Lebensdauer der Oberflächenzustände von Au(111) bei der Fermienergie. 
Ähnliche untere Grenzwerte für die Kohärenzlänge bei der Fermienergie konnten von L. Bürgi auf den Systemen $\mathrm{Cu}(111)$ und $\mathrm{Ag}(111)$ gefunden werden [Bür 4] Diese und weitere aus STM-Messungen erhaltene Daten sind in Abb. 8.30 zusammen mit den hier gefundenen Ergebnissen zusammengefasst. Zwecks besserer Vergleichbarkeit werden alle Ergebnisse mittels Gl. (8.9) in mittlere Lebensdauern konvertiert.

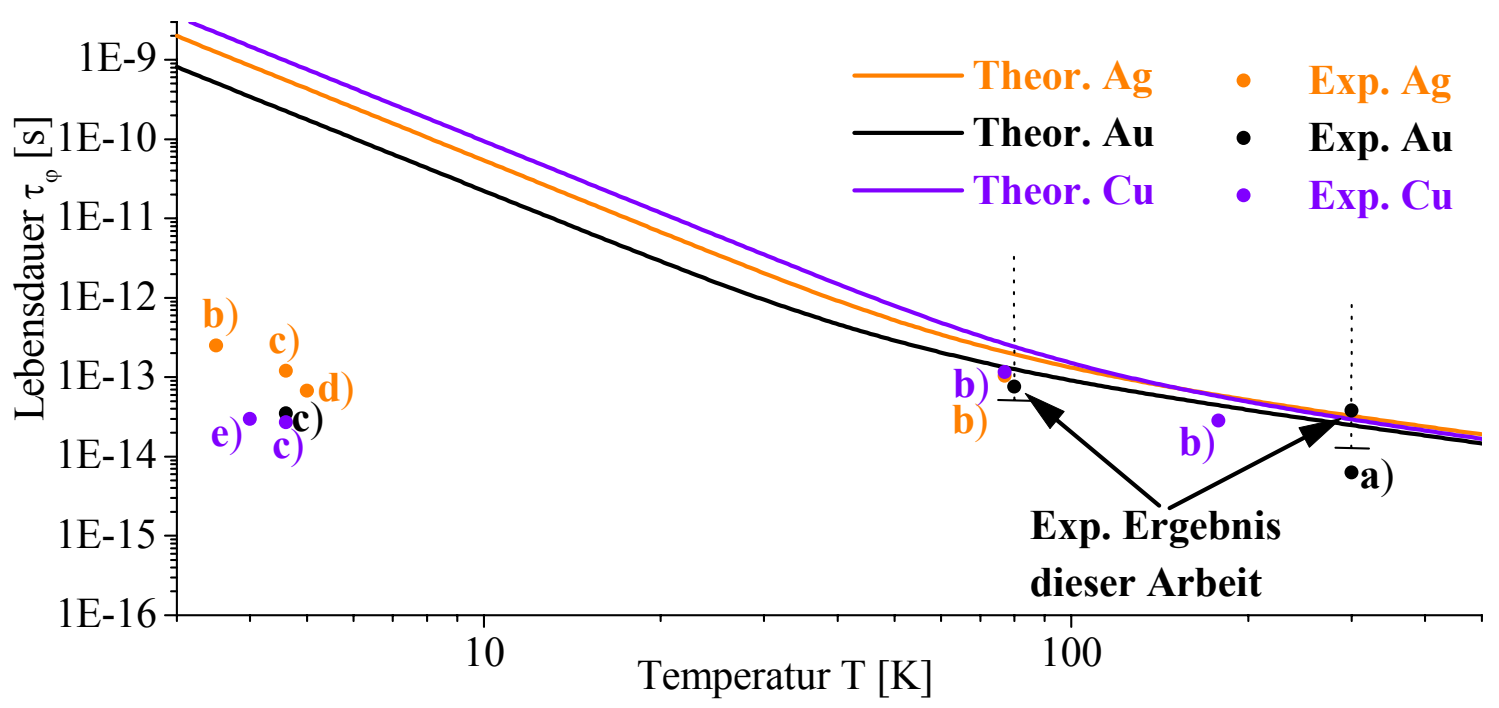

Abb. 8.30 Experimentell mittels STM bestimmte untere Grenzen für die Lebensdauer von Quasiteilchen bei der Fermienergie für die Oberflächenzustände von $\mathrm{Ag}(111), \mathrm{Au}(111)$ und $\mathrm{Cu}(111)$ im Vergleich zu theoretischen Werten für e-ph-Streuung. a) Ref.[Has 1], b) Ref. [Bür 4], c) Ref.[Kli], d) Ref. [Ber, Li 5], e) Ref. [Cro 2]

Die hier bestimmten unteren Grenzwerte für die Lebensdauern liegen für $\mathrm{T}=295 \mathrm{~K}$ mit $\tau_{\varphi}=13 \mathrm{fs}$ um einen Faktor zwei unterhalb des Theoriewertes $\tau_{\varphi}=25 \mathrm{fs}$; der beste Fit-Wert von $\tau_{\varphi}=38 \mathrm{fs}$ liegt jedoch bereits darüber. Bei $80 \mathrm{~K}$ liegen sowohl der experimentell bestimmte untere Grenzwert von $\tau_{\varphi}=50$ fs wie auch der beste Fit-Wert von $\tau_{\varphi}=80$ fs noch unterhalb des theoretisch erwarteten Wertes von $\tau_{\varphi}=130$ fs. 


\section{Thermoelektrische Effekte in der Topografie}

Bei genauerer Betrachtung der Topografien, welche zum einen im Standard-Topografiemodus und zum anderen mittels Raster-Thermospannungs-Mikroskopie gewonnen wurden, fällt ein Unterschied bezüglich dem Interferenzmuster auf.

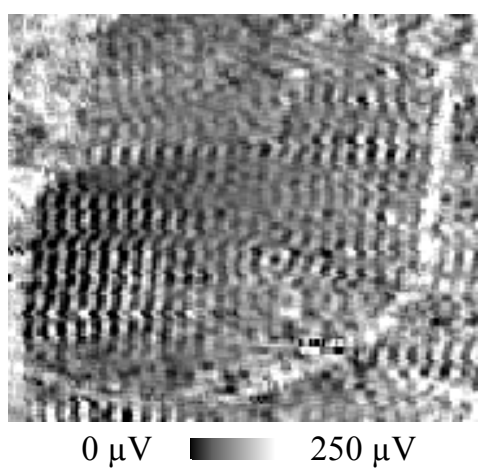

Abb. 9.1 (a) Thermospannung

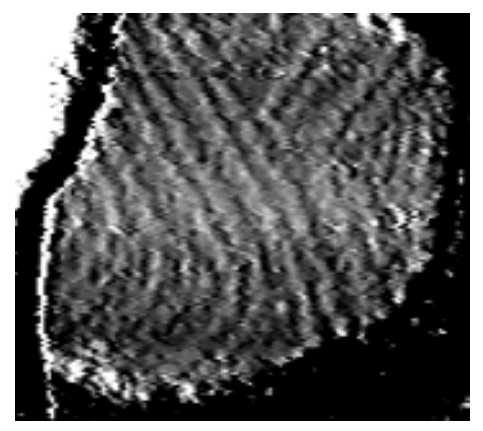

$0 \mathrm{~nm}$

(b) Simultan zur

Thermospannung aufgenommene Topografie

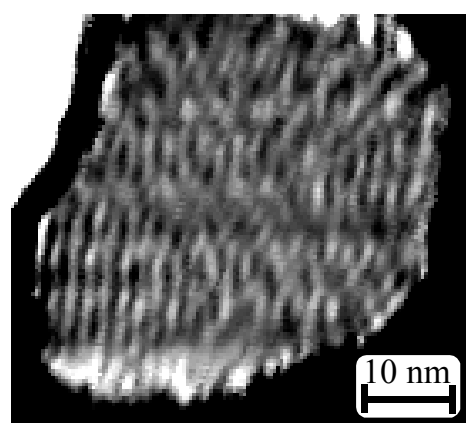

$0 \mathrm{~nm}$ $20 \mathrm{pm}$

c) Topografie derselben Insel ohne simultane Thermospannungsmessung ("Standard-Topografie")

In Abb. 9.1 ist dieselbe Insel abgebildet; in (a) ist die Thermospannung, in (b) die simultan mit der Thermospannung gemessene Topografie und in (c) eine unabhängig dazu durchgeführte alleinige „Standard“-Topografiemessung dargestellt. Die Thermospannungsmessung zeigt ein deutlich $\mathrm{zu}$ erkennendes Interferenzmuster. In der simultan durchgeführten Topografieaufnahme (b) ist dieses Interferenzmuster nur sehr schwach, wenn überhaupt, erkennbar. Es wird im wesentlichen vom Rekonstruktionsmuster überdeckt. Erstaunlicherweise tritt jedoch das in der Thermospannung $\mathrm{zu}$ sehende Interferenzmuster überaus deutlich in einer unabhängig durchgeführten StandardTopografiemessung (c) zutage. Mit seinem Kontrast dominiert das Interferenzmuster sogar über das Rekonstruktionsmuster. Ein derart hoher Kontrast mit Amplituden bis zu 10 pm bei $\mathrm{T}=80 \mathrm{~K}$ ist in der Literatur bisher nicht beobachtet worden.

Die Erklärung für dieses Phänomen liegt im thermoelektrischen Effekt in Kombination mit der technischen Durchführung der Topografieaufnahme. Bei der StandardTopografiemessung wird extern ein Spannungspotenzial $U_{B i a s}$ an die Probe und ein Spannungspotenzial $U_{\text {Spitze }}=0$ an die Spitze angelegt. Die Höhe der Spitze wird so gesteuert, dass der Strom einen definierten Sollwert $\mathrm{I}_{\text {soll }}$ annimmt.

Demgegenüber funktioniert die Topografieregelung im Potenziometrieverfahren leicht abgewandelt: Die Spitze liegt nicht (wie im Standard-Verfahren) auf Massepotenzial, sondern auf dem ortsabhängigen Potenzial $\mathrm{U}_{\mathrm{Th}}(\mathrm{x})$, welches identisch zur lokalen Thermospannung ist. 
Während also bei der Standard-Topografieregelung eine konstante Spannung $U_{\text {Bias }}$ am Tunnelkontakt anliegt, beträgt diese bei der Topografieregelung im Potenziometriemodus $\mathrm{U}_{\mathrm{Bias}}-\mathrm{U}_{\mathrm{Th}}(\mathrm{x})$.

Bei der Standard-Topografie tritt aufgrund des thermoelektrischen Effektes ein ortsabhängiger Diffusionsstrom auf. Dieser Thermo-Tunnelstrom wird alleine durch eine Variation der Spitzenhöhe ausgeglichen. Das Interferenzmuster, welches im Potenziometrieverfahren in der Thermospannung auftreten würde, erscheint also im StandardTopografiemodus direkt in der Topografie. Demgegenüber wird bei Topografieaufnahmen im Potenziometriemodus der thermische Diffusionsstrom durch Anlegen eines Gegenpotentials $U_{T h}(x)$ an die Spitze kompensiert.

Konkreter beschreibbar ist dieser Effekt bei einer Betrachtung im Tersoff-Hamann Formalismus. Zur Vereinfachung wird angenommen, dass sich die LDOS der Oberfläche in einer Form $\rho_{\mathrm{P}}(\overrightarrow{\mathrm{r}}, \varepsilon)=\mathrm{A} \cdot \exp (-\alpha \cdot \mathrm{z}) \cdot \rho_{\mathrm{P}}(\mathrm{x}, \mathrm{y}, \varepsilon)$ beschreiben lässt, d.h. die LDOS soll unabhängig von der Energie $\varepsilon$ mit einer Abklingkonstante $\alpha$ zum Vakuum hin abklingen. Wie im Anhang D gezeigt ist, kann dann für die Topografieregelung im Potenziometriemodus die Topografiespur z(x) senkrecht zur Stufe beschrieben werden zu

$$
\mathrm{z}(\mathrm{x})=\mathrm{z}_{0}+\frac{1}{\alpha} \cdot \ln \left(1-\mathrm{C} \cdot \mathrm{J}_{0}\left(2 \mathrm{k}_{\mathrm{F}} \mathrm{x}\right) \cdot \mathrm{h}_{\mathrm{N}}(\mathrm{x})\right)
$$

Das Potenziometrieverfahren eliminiert jeden thermoelektrischen Effekt in der topografischen Abbildung und bildet die Oberfläche so ab, wie sie auch bei Temperaturgleichheit von Spitze und Probe aussehen würde.

Demgegenüber wird Standard-Topografiemodus eine Topografiespur hergeleitet, welche von der Thermospannung $\mathrm{U}_{\mathrm{Th}}(\mathrm{x})$ beeinflusst wird:

$$
\mathrm{z}=\mathrm{z}_{0}+\frac{1}{\alpha} \cdot \ln \left(\frac{1-\mathrm{C} \cdot \mathrm{J}_{0}\left(2 \mathrm{k}_{\mathrm{F}} \mathrm{x}\right) \cdot \mathrm{h}_{\mathrm{N}}(\mathrm{x})}{\left|1-\mathrm{U}_{\mathrm{Th}}(\mathrm{x}) / \mathrm{U}_{\text {Bias }}\right|}\right)
$$

Die Topografie hängt also davon ab, welche Größenordnung die angelegte Tunnelspannung relativ zur Thermospannung besitzt. Wenn $\left|\mathrm{U}_{\mathrm{Th}}\right| \ll\left|\mathrm{U}_{\text {Bias }}\right|$, dann geht Gleichung (9.2)] über in Gleichung (9.1) In diesem Fall unterscheiden sich die Topografieaufnahmen in beiden Modi nicht voneinander. Wenn allerdings $U_{T h}$ in die Größenordnung von $U_{\text {Bias }}$ gelangt, dann wird in der Topografie die lokale Thermospannung abgebildet.

Die letzte Gleichung führt zu einer interessanten neuen „Betriebsart“ des STM. Für eine genügend hohe Thermospannung $\mathrm{U}_{\mathrm{Th}}$ und entsprechend gewählter kleiner Tunnelspannung $\mathrm{U}_{\text {Bias }}$ sollte es möglich sein, die Interferenzmuster mit im Prinzip beliebig hoher topografischer Amplitude abzubilden. Ein Beispiel hierfür ist in Abb. 9.1 (c) vorgestellt; die 
Maximalamplitude der Thermospannung ist hier $\mathrm{U}_{\mathrm{Th}} \approx 250 \mu \mathrm{V}$, während die angelegte Tunnelspannung $\mathrm{U}_{\text {Bias }}=1000 \mu \mathrm{V}$ beträgt. Für $\mathrm{Au}(111)$ werden experimentell effektive Barrierenhöhen von $\Phi \approx 5 \mathrm{eV}$ gefunden, welche relativ unabhängig vom Abstand $\mathrm{z}$ sind [Ole] Eingesetzt ergibt dies für die Konstante $\alpha=\sqrt{8 \mathrm{~m}_{\mathrm{e}} \Phi} / \hbar=2.3 \AA^{-1}$.

In Abb. 9.2 ist die theoretisch erwartete Topografie im Vergleich zu einem Schnitt durch die experimentellen Daten in Abb. 9.1 (c) für die Standard-Topografiemessung dargestellt. Sowohl die Periodizität als auch die Größenordnung der Amplitude, welche im Falle der reinen Topografiemessung nahezu konstant ist, stimmen gut miteinander überein.

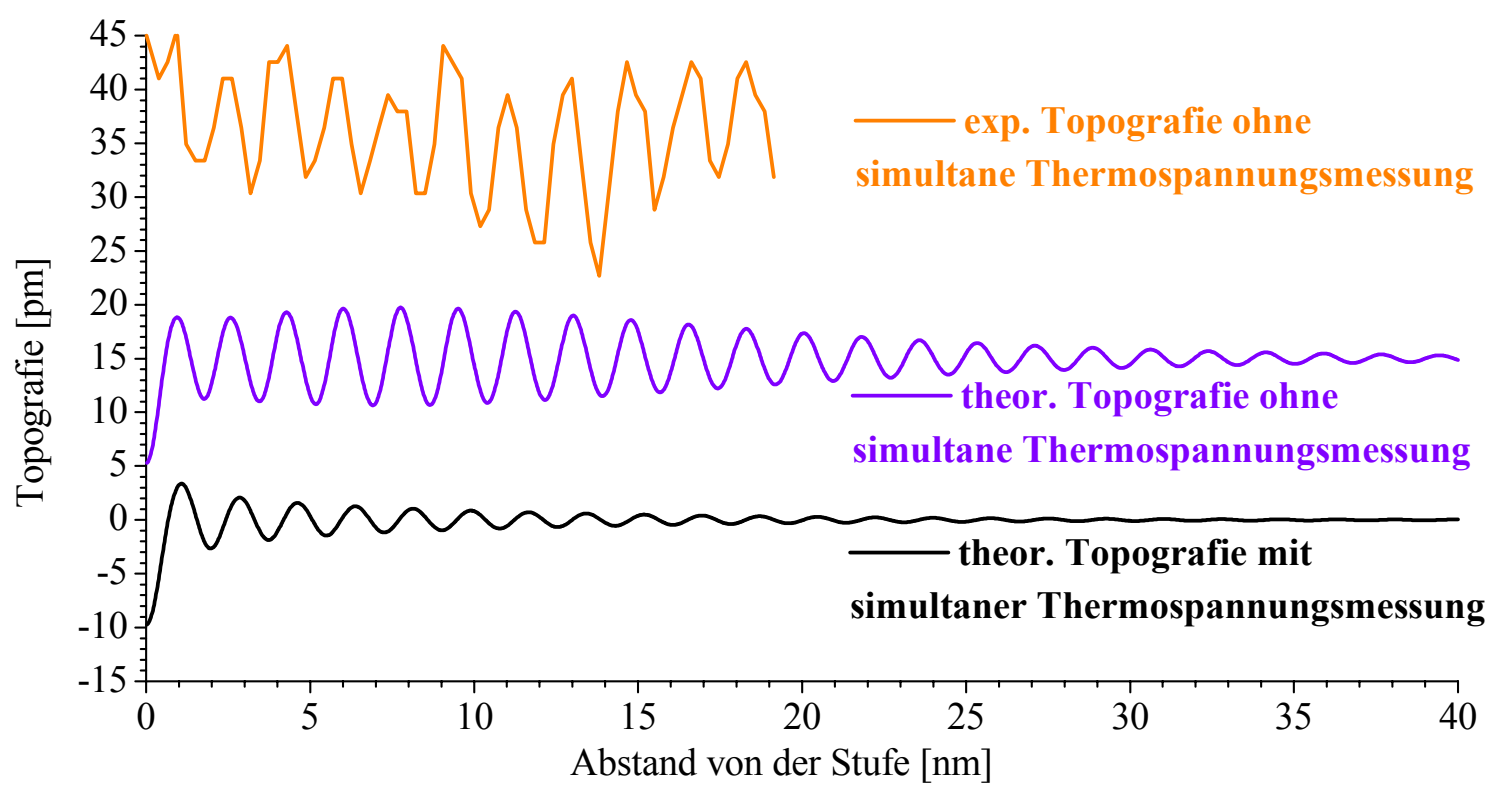

Abb. 9.2 Experimentelle „Standard“-Topografiemessung ohne Kompensation der Thermospannung ( — im Vergleich zur theoretisch erwarteten Topografie mit (-) und ohne (-) simultane Thermospannungsmessung. Für die theoretische Berechnung wurden als Parameter $\mathrm{U}_{\text {Bias }}=1 \mathrm{mV}$, $\mathrm{T}_{\mathrm{P}}=110 \mathrm{~K}, \mathrm{~T}_{\mathrm{S}}=155 \mathrm{~K}$ und $\mathrm{C}=0.2$ verwendet 29 .

In der Praxis ist ein Kartieren von Interferenzmustern in der Topografie mit Hilfe des thermoelektrischen Effektes nur sinnvoll möglich, wenn hohe Temperaturdifferenzen, also auch hohe Thermospannungen vorhanden sind, welche in der Größenordnung um die $100 \mu \mathrm{V}$ mit der Tunnelvorspannung vergleichbar sind. Es muss jedoch darauf geachtet werden, dass die Thermospannung (welche sich aus einem Offsetwert und den ortsabhängigen Oszillationen zusammensetzt) den $\mathrm{U}_{\text {Bias }}$-Wert nicht überschreitet, da ansonsten der Tunnelstrom sein Vorzeichen umkehrt und die Spitze in die Probe hineinfährt.

Um die Bedeutung dieses Effektes zu verdeutlichen, ist in Abb. 9.3 eine Simulation der Topografie für verschiedene Probenvorspannungen $U_{\text {Bias }}$ dargestellt.

\footnotetext{
${ }^{29}$ Die Konstante $\mathrm{C}=\mathrm{P} \cdot \mathrm{R}$ ist hier aus der Reflektivität $\mathrm{R}=0.4$ der Stufe [Sch 1] und dem Anteil $\mathrm{P}=0.5$ der Oberflächenzustände am gesamten Tunnelstrom [Kli, Che 1, Bür 1] zusammengesetzt.
} 

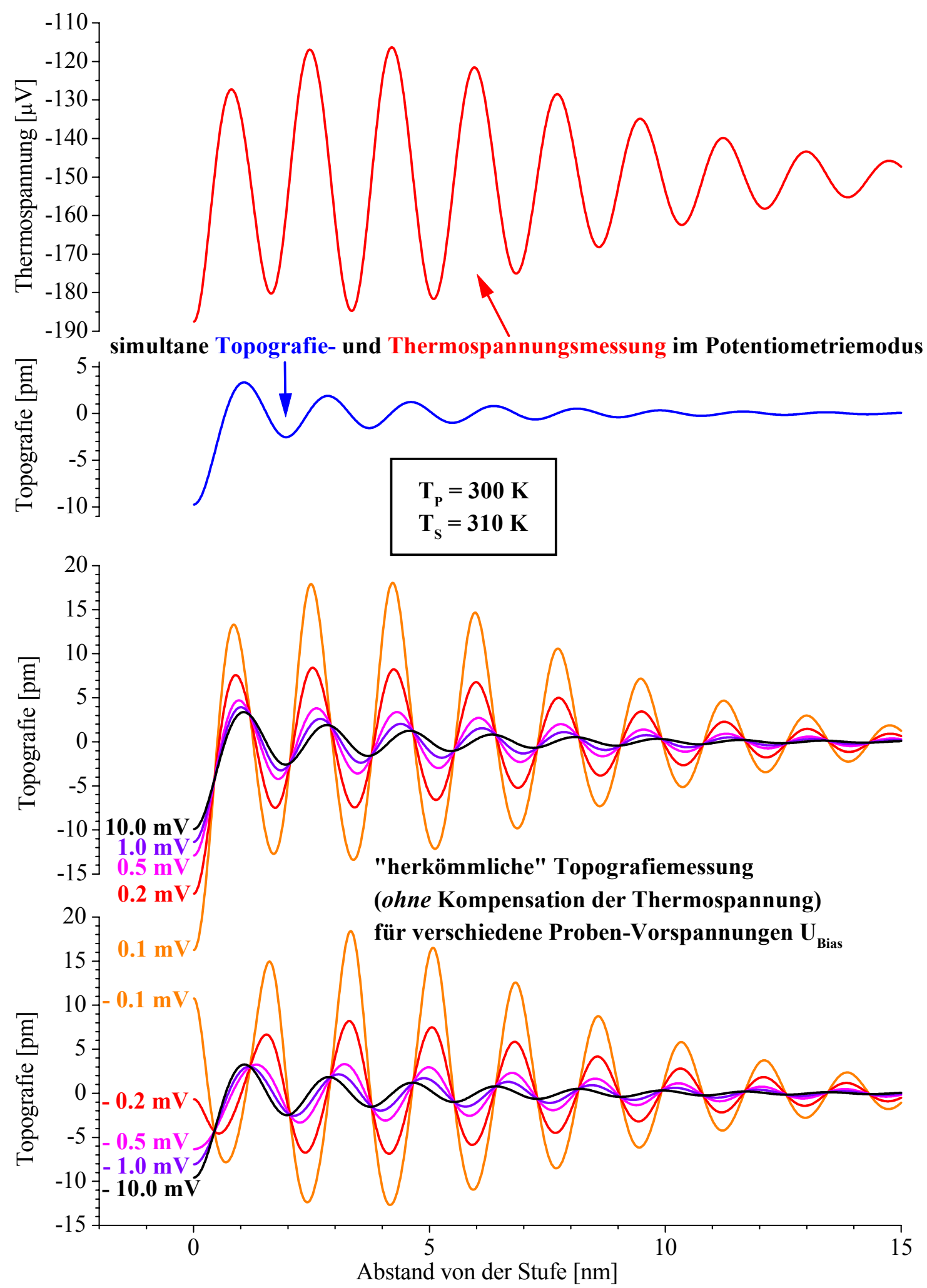

Abb. 9.3 Thermoelektrischer Effekt in der Topografiemessung für $T_{P}=300 \mathrm{~K}, \mathrm{~T}_{\mathrm{S}}=310 \mathrm{~K}$ und $\mathrm{C}=0.2$. Obere Graphen: Topografie (_ - ) und Thermospannung (_ ${ }_{-}$im Potenziometriemodus. Die Topografie ist weitestgehend unabhängig von der extern angelegten Probenvorspannung $U_{\text {Bias, }}$, da Thermospannungen über Variation des Potenzials $U_{\text {Spitze }}=U_{\mathrm{Th}}(\mathrm{x})$ kompensiert werden. Untere Graphen: Topografie im ,herkömmlichen“ Topografiemodus für verschiedene $U_{\text {Bias. }}$. Da in diesem Fall das Potenzial der Spitze konstant $\mathrm{U}_{\text {Spitze }}=0$ ist, müssen thermische Diffusionsströme über die Abstandsregelung ausgeglichen werden. Bei kleinen Biasspannungen erscheint mit ungewöhnlich hoher topografischer Amplitude dasselbe Interferenzmuster, welches alternativ bei Potentriometriemessungen in der Thermospannung gefunden wird. 
Die Simulationen zeigen, dass bei Vorhandensein einer Temperaturdifferenz zwischen Spitze und Probe die topografischen Effekte in ihrer Amplitude um ein Vielfaches zunehmen können. Bei Raumtemperatur reicht z.B. schon eine Temperaturdifferenz von $10 \mathrm{~K}$ aus, um in der Topografie bei einer Tunnelspannung von $0.2 \mathrm{mV}$ Amplituden bis zu $0.25 \AA$ zu finden.

Der Vorteil bei der Nutzung der Thermospannung für den Topografiemodus liegt darin, dass kein spezieller experimenteller Aufbau für die Potenziometrie nötig ist und somit im Prinzip jedes herkömmliche STM benutzt werden kann, so lange eine genügend große Temperaturdifferenz zwischen Spitze und Probe erzeugt wird. Dieser Effekt beschränkt sich nicht alleine auf die Beobachtung von Interferenzmustern. Die Sensitivität der Thermospannung auf die differentielle lokale Zustandsdichte könnte potenziell Anwendungen wie chemische Kartierung, Unterscheidung magnetischer Domänen o.ä. allein durch topografische Messungen ermöglichen.

In der Literatur könnte dieser Effekt eine Erklärung für einige Phänomene liefern, welche bisher noch nicht überzeugend interpretiert werden konnten. Als Beispiel seien hier die sog. „Giant-Friedel-Oscillations“ auf dem System Be(0001) genannt, welche von P. T. Sprunger und Mitarbeitern beobachtet wurden [Pet 5]. Die Autoren benutzten ein variables Tieftemperatur-STM bei einer Temperatur von $150 \mathrm{~K}$ bis $\mathrm{zu} 300 \mathrm{~K}$ und studierten das Interferenzmuster, welches von einem zweidimensionalen Elektronengas von Oberflächenzuständen herstammt. Sie beobachteten, dass die Amplitude des Interferenzmusters bei Erniedrigung der Tunnelspannung von $-35 \mathrm{mV}$ auf $-2.1 \mathrm{mV}$ um einen Faktor zehn von $4.2 \mathrm{pm}$ auf $42 \mathrm{pm}$ zunahm, und das Interferenzmuster für diesen Fall auf der gesamten Oberfläche mit relativ konstanter Amplitude zu sehen war. Als Ursache wurde ein Vielteilcheneffekt vermutet. Die hier besprochenen topografischen Effekte bieten eine alternative Erklärung für die von den Autoren angegebenen Parameter an, zumal ein größerer Temperaturunterschied zwischen Spitze und Probe bei einem variablen Tieftemperatur-STM durchaus wahrscheinlich sind. 


\section{Monatomare Stufen als Beugungsgitter}

\subsection{Einführung}

Im Gegensatz zu allen anderen (111)-Edelmetalloberflächen besitzt die Au(111)-Oberfläche die sogenannte „Herringbone-Rekonstruktion“. Die Umordnung der Atome ist mit einer Veränderung der elektronischen Struktur von der Oberfläche korreliert; es wird ein Confinement-Potenzial für elektronische Zustände geschaffen. Zusammen mit der zweiten Besonderheit der $\mathrm{Au}(111)$-Oberfläche, nämlich der Existenz von Oberflächenzuständen, eröffnet sich hier ein interessantes System für die Erforschung quantenmechanischer Effekte, über das bisher erstaunlich wenig in der Literatur berichtet wird.

Fokus der bisherigen Untersuchungen ist die Frage, welche Wechselwirkung die Oberflächenzustände mit der Rekonstruktion eingehen. Hierzu gibt es zwei Zugänge: Zum einen den rein topografischen Zugang, welcher erstmals 1996 von D. Fujita in der Gruppe um H. Nejoh untersucht wurde [Nej, Fuj 1-3]. Die experimentellen Ergebnisse deuteten die Autoren dahingehend, dass die Rekonstruktion als eindimensionales Confinement-Potenzial für die Oberflächenzustände wirkt.

Zum anderen gibt es den spektroskopischen Zugang, welcher erstmals 1998 von W. Chen in der Gruppe um M. F. Crommie beschritten wurde [Che 1], Die spektroskopischen Daten deuteten darauf hin, dass das Rekonstruktions-Potenzial so schwach moduliert ist, dass Elektronen in der Nähe der Fermienergie keinerlei Confinement mehr erfahren sollten.

Dieser Widerspruch zwischen topografischen und spektroskopischen Daten wurde in der Literatur bisher noch nicht näher behandelt.

\subsection{Die Rekonstruktion: Confinement für Oberflächenzustände?}

Eine erste theoretische Untersuchung der elektronischen Struktur der Au(111)-Oberfläche, welche die verschiedenen Stapelungsmöglichkeiten der obersten Atomlage in Betracht zieht, wurde 1991 von N. Takeuchi und Mitarbeitern durchgeführt [Tak] Die Autoren konnten eine Ladungsdichteverteilung des Oberflächenzustandes für eine fcc-, hcp- und Übergangsbereichs-Stapelung bestimmen (Abb. 10.1). 


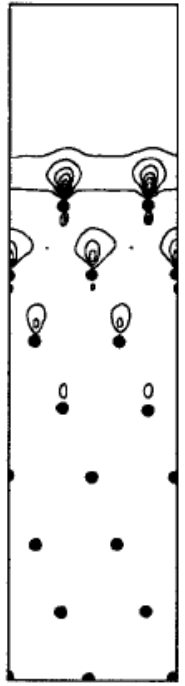

fcc

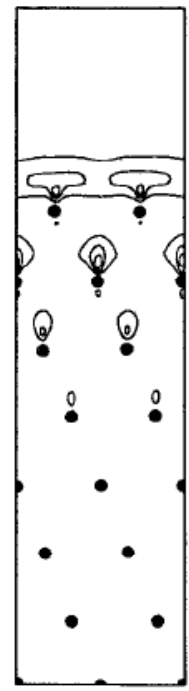

Übergang

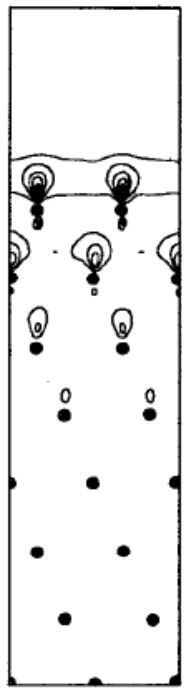

hcp
Abb. 10.1 Ladungsdichteverteilung des Oberflächenzustandes von $\mathrm{Au}(111)$ für verschiedene Stapelungsformen der obersten Atomlage. Mit „Zwischengitter" wird hier eine Atomstapelung beschrieben, bei der die Atome der obersten Atomlage genau zwischen einer fcc- und hepStapelposition (=Übergangsbereich) liegen (aus Ref. N. Takeuchi [Tak]].

Obwohl die Detailform der Rekonstruktion nicht berücksichtigt wurde, deuten diese Ergebnisse an, dass zwischen der fcc- und der hcp-Region keine Unterschiede in der Ladungsdichte $\mathrm{zu}$ erwarten sind. Lediglich im Übergangsbereich bei den Rekonstruktionslinien erwartet man eine schwächere Präsenz der Oberflächenzustände.

Erste experimentelle Ergebnisse in Form von rein topografischen Messungen des Interferenzmusters an einer Stufenkante wurden 1996 von D. Fujita in der Gruppe um H. Nejo veröffentlicht [Nej, Fuj 1-3]. Im Gegensatz zur theoretischen Erwartung beobachteten die Autoren eine deutliche Erhöhung der Amplitude der stehenden Wellen an den Rekonstruktionslinien (=,Soliton-Wällen“) sowie in der hcp-Region relativ zu der der fccRegion (s. Abb. 10.2
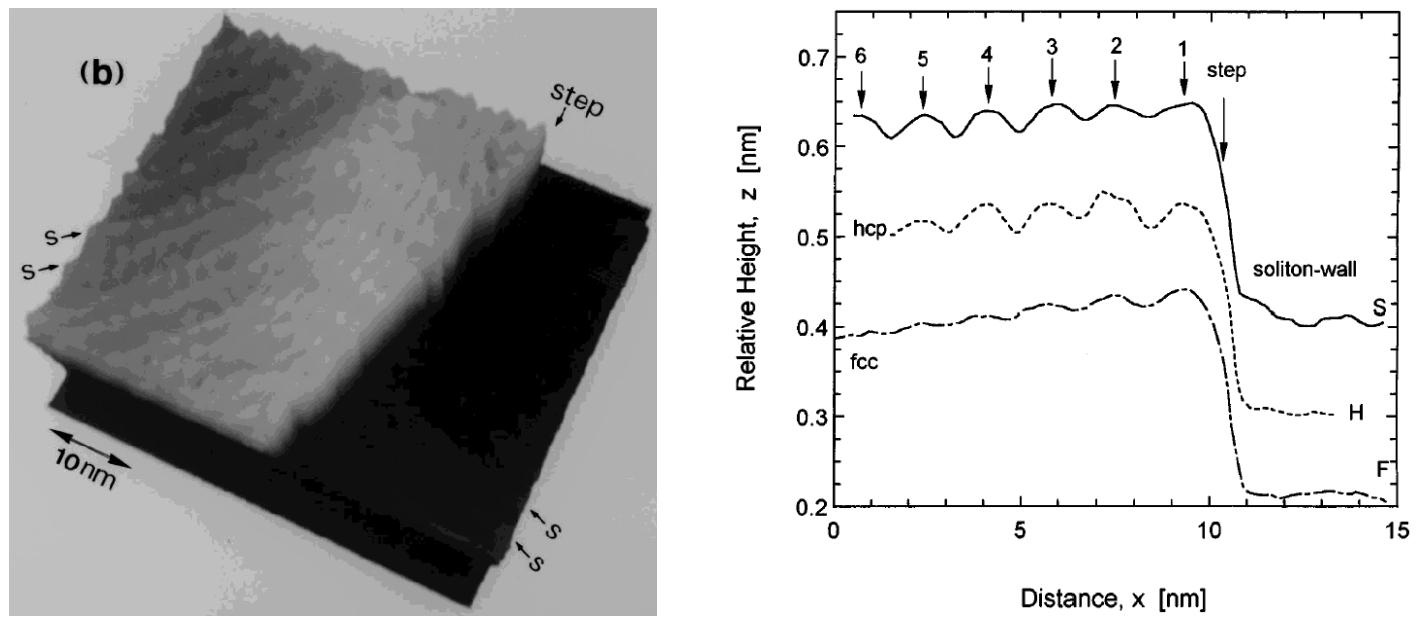

Abb. 10.2 Topografie (links) und Querschnitte (rechts) senkrecht zur Stufenkante entlang den Domänen der Rekonstruktion (aus Ref. D. Fujita [Fuj 1]) 
Dies wurde interpretiert in der Form, dass durch Confinement die Elektronenwellen in einem durch die Rekonstruktion vorgegebenem Oberflächenpotenzial entlang der Soliton-Wälle ,geleitet" werden.

Neue Erkenntnisse über das Oberflächenpotenzial konnten 1998 durch ortsaufgelöste dI/dUspektroskopische Messungen mit dem STM von W. Chen und Mitarbeitern in der Gruppe um M. F. Crommie erbracht werden [Che 1] Abb. 10.3.
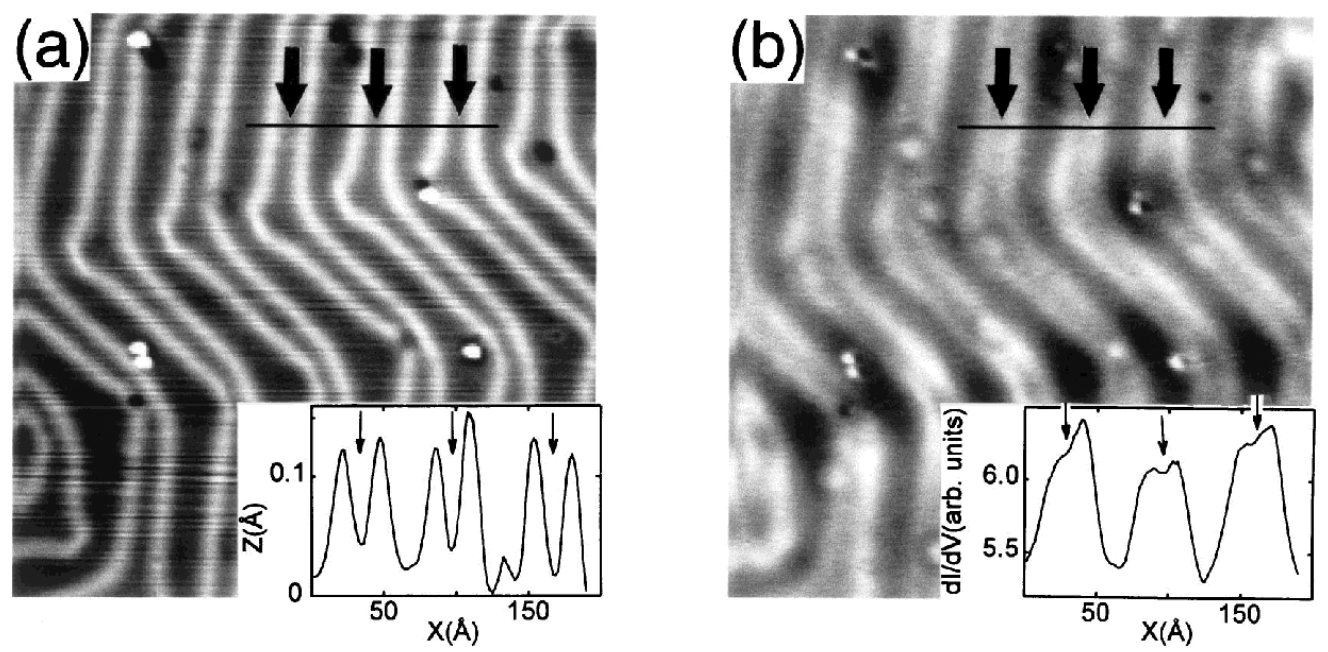

Abb. 10.3 Topografie (links) und ortsaufgelöstes dI/dU-Signal (rechts) für eine Tunnelspannung von $-0.48 \mathrm{~V}$ (aus Ref. W. Chen [Che 1]

Die Autoren beobachteten, dass die Oberflächenzustände für relativ tiefe Energien von der Bandunterkante bei $-0.52 \mathrm{eV}$ bis $\mathrm{zu}-0.43 \mathrm{eV}$ relativ zur Fermienergie $\mathrm{E}_{\mathrm{F}}$ in der hcp-Region lokalisiert sind. Diese Beobachtung kann in einem zweidimensionalen Modell durch ein Oberflächenpotenzial erklärt werden, welches die Form eines mit $25 \mathrm{meV}$ hohen Stufen modulierten Kronig-Penney Potenzials hat Elektronen oberhalb des angegebenen Energiebereiches sollten nur noch sehr geringe Lokalisierung durch dieses Potenzial erfahren.

Diese Beobachtung wurde 2000 von L. Bürgi in der Gruppe um K. Kern bestätigt [Bür 1], welcher ebenfalls ortsaufgelöst dI/dU-Spektroskopie an der Rekonstruktion der Au(111)Oberfläche durchführte und das Ein-Teilchen-Potenzial der Rekonstruktion mittels LindhardAbschirmungstheorie aus der Ladungsdichteverteilung berechnen konnte. Auch L. Bürgi beobachtete oberhalb einer Energie von $-0.3 \mathrm{eV}$ unterhalb von $\mathrm{E}_{\mathrm{F}}$ keine Lokalisierung der Ladungsdichte der Oberflächenzustände bezüglich der Rekonstruktion.

Zusammengefasst kann davon ausgegangen werden, dass Elektronen nahe von $\mathrm{E}_{\mathrm{F}}$ keine Wechselwirkung mit der Rekonstruktion zeigen. Dies wirft jedoch die Frage auf, warum

\footnotetext{
${ }^{30}$ Angesichts der Tatsache, dass sich die Rekonstruktion nur auf die oberste Atomlage beschränkt [Har], die Oberflächenzustände aber auch noch einige Atomlagen weit im Kristallinnern vorzufinden sind [Liu, Tak], beschreibt das zweidimensionale Potenzialmodell die Experimente relativ erfolgreich.
} 
trotzdem in der Topografie in den beiden Rekonstruktionsdomänen verschiedene Amplituden für die stehenden Wellen gefunden werden [Nej, Fuj 1-3].

Die Frage, ob Elektronenwellen sich entlang von Rekonstruktionskanälen orientieren, wird auch im Rahmen dieser Arbeit behandelt. Dazu wird eine kombinierte Topografie- und Thermospannungsmessung in der Nähe einer Stufe verwendet und mittels Autokorrelation und Fouriertransformation analysiert wie in Abb. 10.4 gezeigt. Die Topografie zeigt die Herringbone-Rekonstruktion sowie etwas schwächer auf der linken Seite auch stehende Wellen, welche einer (außerhalb der Abbildung liegenden) monatomaren Stufe zuzuordnen sind. In der Autokorrelation der Topografie sind deutlich zwei Richtungen der Rekonstruktionslinien wiederzuerkennen, welche zum einen senkrecht zur monatomaren Stufe wie auch im $60^{\circ}$-Winkel dazu nach rechts unten weisen. Auch die Fouriertransformation zeigt eine x-förmige Struktur, welche dem Rekonstruktionsmuster zuzuordnen ist. 

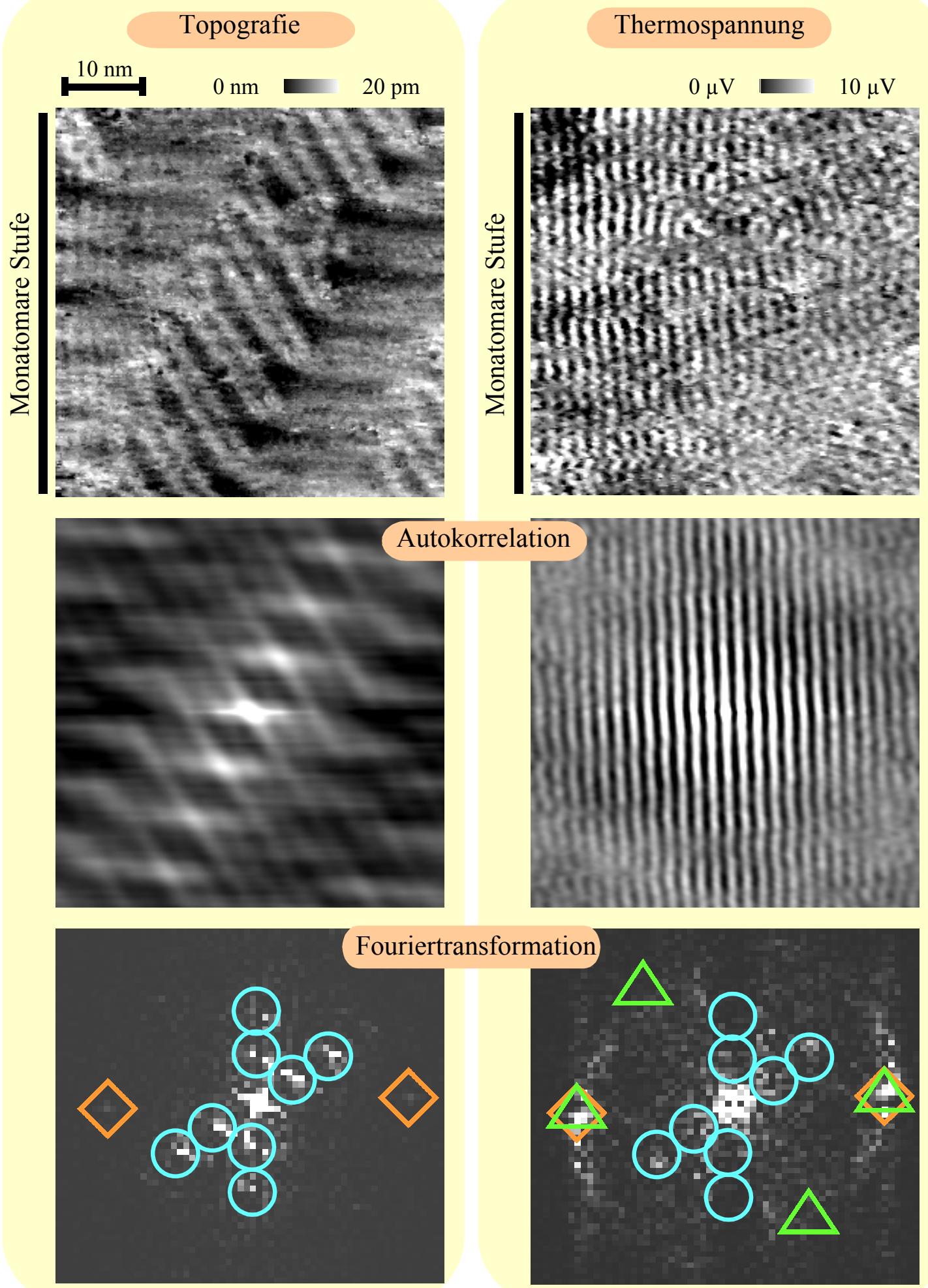

Abb. 10.4 Autokorrelation und Fouriertransformation einer kombinierten Topografie- (linke Spalte) und Thermospannungsmessung (rechte Spalte). Die streuende monatomare Stufe verläuft in y-Richtung außerhalb des Bildbereiches auf der linken Seite. $\mathbf{O}=$ Mit der Rekonstruktion korrelierte Fourierkomponenten. $\boldsymbol{\nabla}$ = Fourierkomponenten von stehenden Wellen parallel zu streuenden Stufe. $\boldsymbol{\Delta}$ = Fourierkomponenten von stehenden Wellen, welche entlang von Rekonstruktionskanälen laufen würden. 
Wenn die Rekonstruktionsdomänen als „Wellenleiter“ wirken würden, dann müsste dies auch in der Autokorrelation und der Fouriertransformation der Thermospannung zu beobachten sein. Diese zeigen aber nur eine Komponente von stehenden Wellen parallel zur Stufenkante, während in die zweite Richtung der Rekonstruktionslinien keinerlei Signal beobachtet wird. Stattdessen zeigt die Fouriertransformation der Thermospannung zusätzlich noch schwach die $\mathrm{x}$-förmige Struktur, welche mit der rein topografischen Struktur der Rekonstruktion übereinstimmt (blaue Kreise in Abb. 10.4.

Zusammengefasst steht diese Analyse im Widerspruch zu der Theorie, wonach die Rekonstruktion als Übergitter für die Oberflächenzustände wirkt, zumindest für Elektronenenergien nahe der Fermienergie.

\subsection{Periodische Modulation monatomarer Stufen}

Bisher wird bei der Betrachtung von Interferenzmustern immer davon ausgegangen, dass die monatomaren Stufen topologisch perfekt gerade sind. Dies ist bei der Berücksichtigung der Rekonstruktion von $\mathrm{Au}(111)$ aber schon selbst dann nicht möglich, wenn die Atome einer Stufenkante in einer perfekten Reihe angeordnet sind. Der Wechsel der Stapelfolgen von fcczur hcp-Region erzwingt, dass die Atome in Richtung der Rekonstruktionslinien um einen Betrag von $\mathrm{a} \cdot \sqrt{3} / 6 \approx 0.083 \mathrm{~nm}$ verschoben sind (s. Kap. 6.3). Dies ist in Abb. 10.5 an einem Beispiel für eine monatomare Stufe in $[1 \overline{1} 0]$-Richtung demonstriert, welche von Rekonstruktionslinien in $[11 \overline{2}]$-Richtung geschnitten wird.

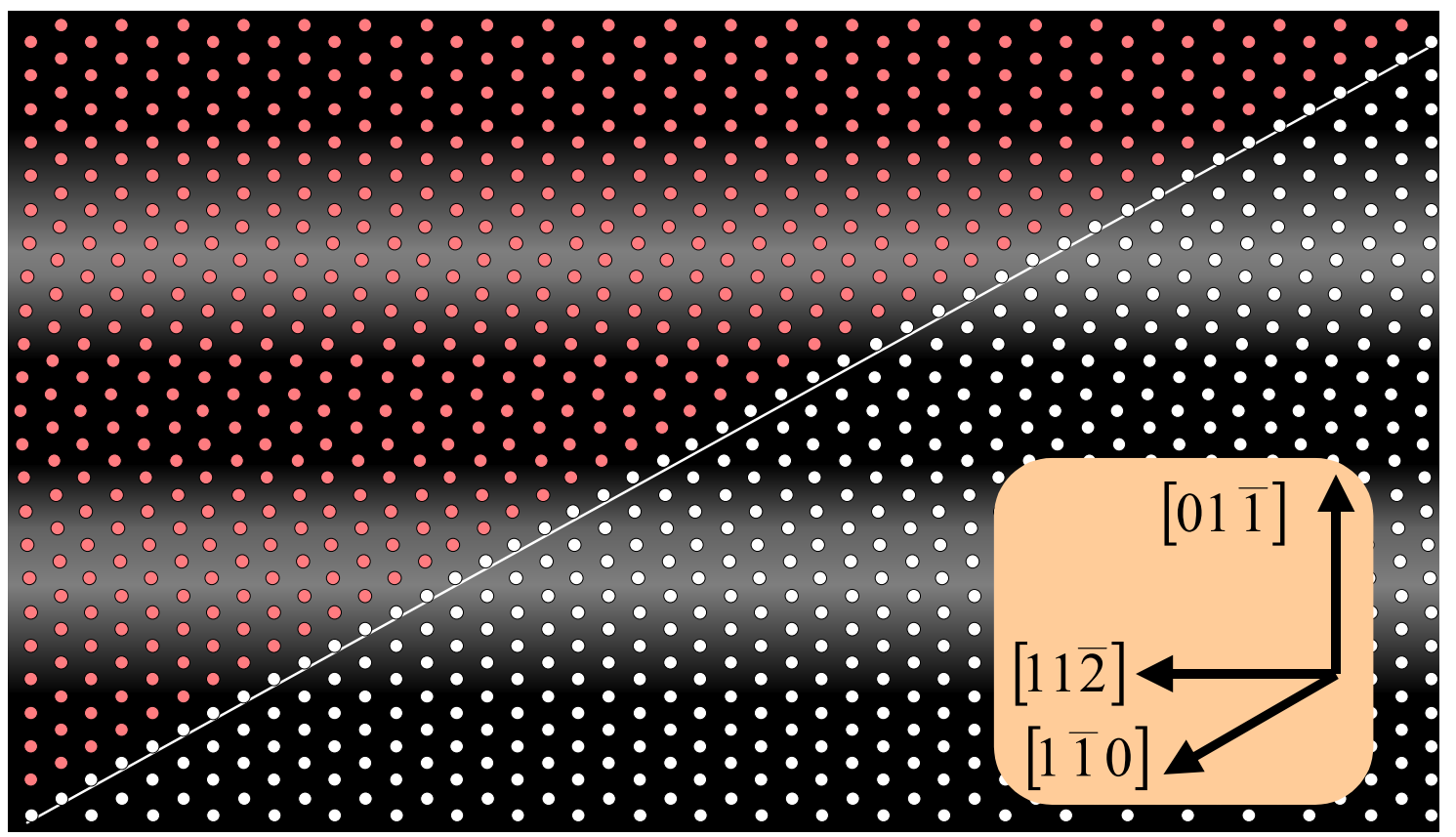

Abb. 10.5 Atomkarte einer monatomare Stufe, welche von der Rekonstruktionslinien „überquert“ wird. Der Wechsel von hcp-Region (Mitte) zur fcc-Region (oben und unten) verursacht eine Modulation der Stufenkante. 
Wie schon in Kap. 6.3 erwähnt, wird die Herringbone-Rekonstruktion nicht durch einzelne Stufen gestört, d.h. das Muster setzt sich über die Stufe hinweg fort.

Im Rahmen dieser Arbeit werden Modulationen an einer Stufenkante gefunden, die deutlich größer als die in Abb. 10.5 konstruierten sind, wie Abb. 10.6 zeigt.
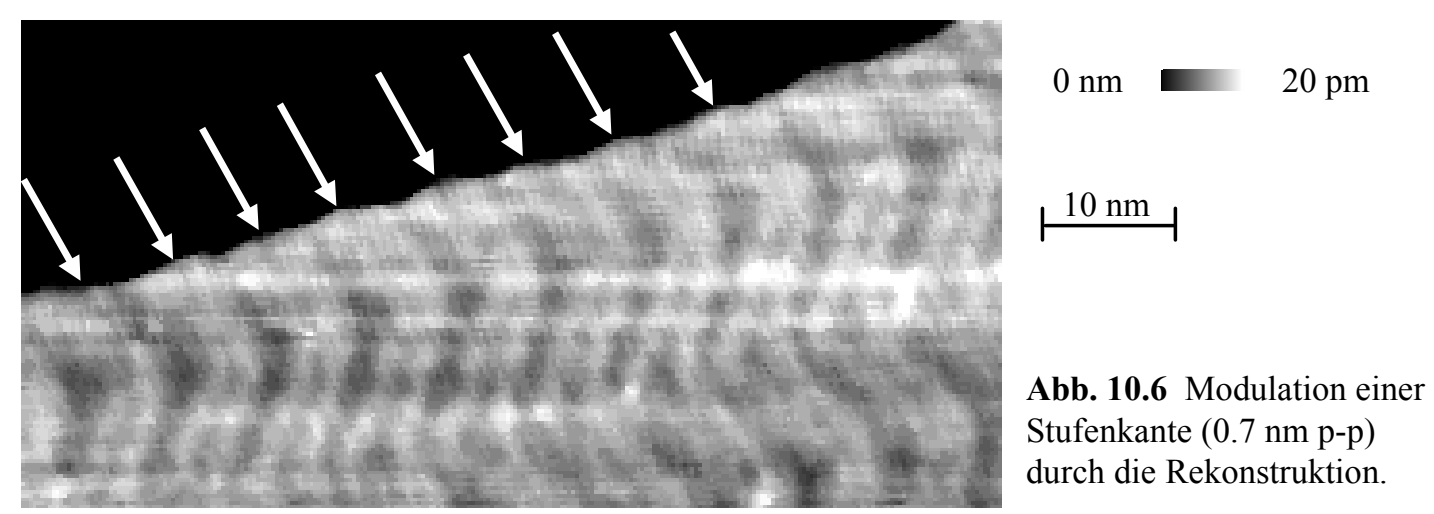

Die Modulation der Stufe beträgt ca. $0.7 \mathrm{~nm}$ p-p, was in Lateralrichtung 2 bis 3 Atomreihen entspricht, und schneidet die Rekonstruktionslinien in einem Winkel von ca. $17^{\circ}$. Die Periodizität der Stufe ist jedoch direkt korreliert mit den Überquerungspunkten der Rekonstruktionslinien und beträgt hier etwa $6.6 \mathrm{~nm}$.

Eine im Rahmen dieser Arbeit durchgeführte systematische Untersuchung von Stufen auf einer getemperten $\mathrm{Au}(111)$-Oberfläche führt zu der Erkenntnis, dass die Stufen in der hcpRegion ausgewölbt sind, zumeist mit einer experimentell gemessenen lateralen Modulation zwischen 0.6 und 1.4 nm p-p, vermutlich abhängig von der Richtung der Stufe.

In der Literatur ist über diesen Effekt bisher noch nicht berichtet worden.

Diese Ergebnisse legen nahe, dass im thermodynamischen Gleichgewichtszustand die Stufen nicht wie ,mit dem Lineal gezogen“" ausseher ${ }^{[1]}$ Vielmehr bringt eine Umordnung der Atome von der fcc-Region in die hcp-Region vermutlich einen Gewinn an Freier Energie ein und begünstigt die Diffusion von der fcc- in die hcp-Region. Die Stufe wird dann in der hcpRegion ,ausgewölbt“. Diese Auswölbung kostet jedoch andererseits Freie Energie, da im atomaren Bild zusätzliche Bindungen zu nächsten Nachbaratomen aufgebrochen werden müssen [Ros] Diese beiden Prozesse konkurrieren gegeneinander, so dass sich im Gleichgewicht eine mit einer gewissen Amplitude lateral modulierte Stufe ergibt.

\footnotetext{
${ }^{31}$ Es kommt noch hinzu, dass bei einer nicht niedrig indizierten Stufe Atomreihen „geschnitten“ werden. Eine eingeschobene Atomreihe der Breite $0.25 \mathrm{~nm}$ ist dann mit einem Sprung in der Stufentopologie verbunden.
} 


\subsection{Interferenzmuster an modulierten Stufen}

Die durch die Rekonstruktion modulierten Stufen besitzen eine laterale Variation (ca. 0.6-1.4 nm p-p), welche größenordnungsmäßig in den Bereich der halben Wellenlänge der Oberflächenzustände $(1.8 \mathrm{~nm})$ fällt. Es sind deshalb Einflüsse auf das Interferenzmuster zu erwarten. Dazu ist in Abb. 10.7 eine kombinierte Topografie und Thermospannungsmessung vorgestellt.
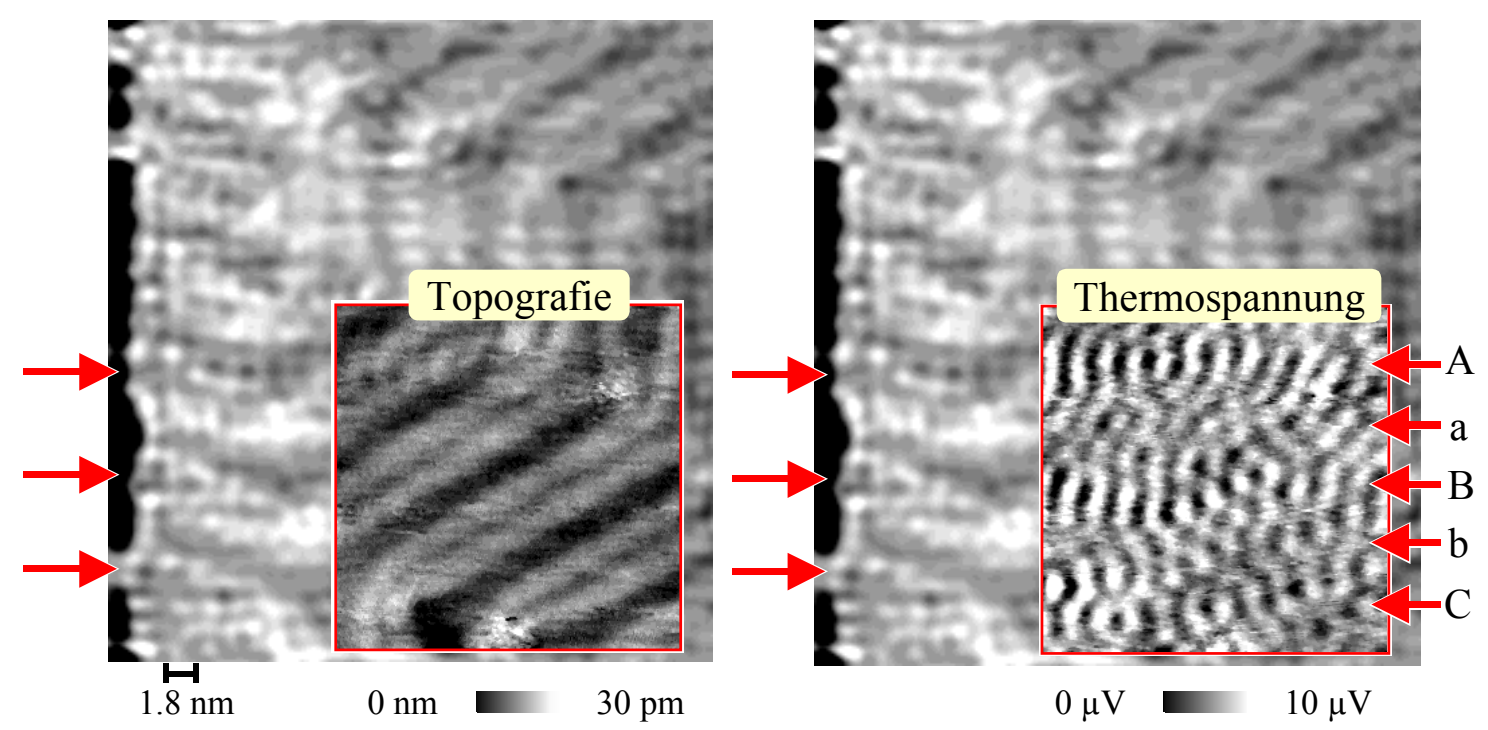

Abb. 10.7 Im Hintergrund ist die Topografie einer modulierten monatomaren Stufe dargestellt. In der rechten unteren Ecke ist innerhalb eines Ausschnittes Topografie und zugehörige Thermospannung gemessen worden. Die linksseitigen Pfeile weisen auf Bereiche, in denen eine hcp-Region der Rekonstruktion die Stufe kreuzt. A,B und C weisen auf Bereiche mit hohem Kontrast (also großer Thermospannungsamplitude), a und b entsprechend auf Bereiche mit schwachem Kontrast.

Im Hintergrund der beiden Messungen in Abb. 10.7 ist eine (identische) Topografie mit einer monatomaren Stufe dargestellt. Die Stufe wird von Rekonstruktionslinien in einem Winkel von ca. $26^{\circ}$ zur Normalen geschnitten. Die Positionen, bei denen eine hcp-Region die Stufe überquert, sind mit einem Pfeil gekennzeichnet; sie sind durch eine Auswölbung der Stufe von $0.8 \mathrm{~nm}$ p-p charakterisiert. Die Periodizität der Auswölbungen beträgt ca. $7 \mathrm{~nm}$. Neben der Rekonstruktion sind in der Topografie auch schwach stehende Wellen zu erkennen.

Anschließend wird eine hochaufgelöste simultane Topografie- und Thermospannungsmessung unmittelbar neben der Stufe durchgeführt. Diese wird in Abb. 10.7 grafisch über die ursprüngliche Topografiemessung gelegt, um eine Korrelation der Thermospannung mit der Topologie der Stufenkante zu ermöglichen.

Die Thermospannung zeigt ein Interferenzmuster, in welchem die typische Periodizität der halben Fermi-Wellenlänge der Oberflächenzustände deutlich hervortritt. Bei genauerem Hinsehen zeigt sich eine zusätzliche Modulation des Interferenzmusters. Die Messung unterteilt sich in drei horizontal ausgedehnte Bereiche mit relativ hohem Kontrast (in Abb. 10.7 markiert mit A, B und C), welche korreliert sind mit den Kreuzungspunkten der 
hcp-Region mit der Stufe. Getrennt sind diese Gebiete jeweils durch einen Bereich, in dem das Interferenzmuster zwar noch sichtbar ist, aber einen deutlich schwächeren Kontrast besitzt (markiert in Abb. 10.7 mit a und b). Dies wird deutlicher in Abb. 10.8. in welcher fünf horizontale Querschnitte durch die jeweiligen Bereiche der Thermospannung gelegt sind und die Einhüllende aufgetragen ist. Die Schnitte zeigen, dass die Oszillationen in A, B und C zumindest im stufennäheren Bereich wesentlich stärker ausfallen als in den Bereichen a und b. Erst weiter weg von der Stufe gleichen sich die Amplituden an.

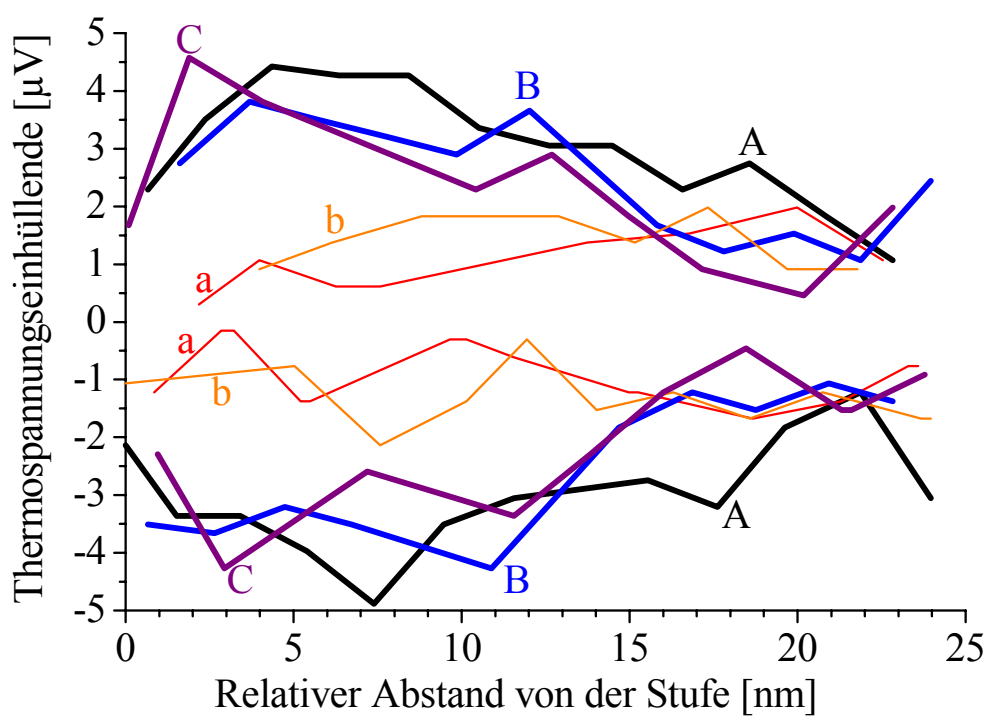

\begin{abstract}
Abb. 10.8 obere und untere Einhüllende von Querschnitten durch die mit $\mathrm{A}, \mathrm{B}$ und $\mathrm{C}$ markierten Bereiche starken Kontrastes (三) sowie durch die mit $\mathrm{a}$ und $\mathrm{b}$ markierten Bereich schwachen Kontrastes (=) in der Thermospannungsmessung aus Abb. 10.7
\end{abstract}

Die Symmetrie und Periodizität dieser Intensitätsvariationen lassen vermuten, dass die Topologie der Stufe das Interferenzmuster entscheidend prägt, also wie ein eindimensionales Gitter für Elektronenwellen wirkt. Dieses Modell wird durch Simulationen, welche im folgenden Abschnitt vorgestellt werden, untermauert.

\title{
10.5. Simulation des Beugungsmusters
}

In diesem Abschnitt wird analysiert, welchen Einfluss eine durch die Rekonstruktion modulierte Stufe auf das Interferenzmuster hat. Hierbei wird vor allem auf folgende Fragestellungen eine Antwort gesucht:

- Gibt es im Interferenzmuster charakteristische Merkmale, welche alleine auf eine periodische Stufenmodulation zurückzuführen sind?

- Wie hängen diese Merkmale von den Parametern der Stufentopologie ab?

- Lassen sich mit diesen Merkmalen experimentell beobachtete Effekte erklären?

Zur Klärung dieser Fragestellungen wird die LDOS numerisch nach dem in Kap. 7.2 angegebenen Verfahren berechnet. 


\title{
10.5.1. Technisches und grafische Darstellung
}

Die Stufe wird hier als unendlich hohe Potenzialbarriere moduliert, an welcher die Elektronenwellen bei der Streuung einen Phasensprung von $-\pi$ erfahren (d.h. sie bilden an der Stufe einen Knoten im stehenden Wellenfeld). Die Topologie der Stufe wird durch hintereinander angeordnete „Elementarzellen“ festgelegt (Abb. 10.9).

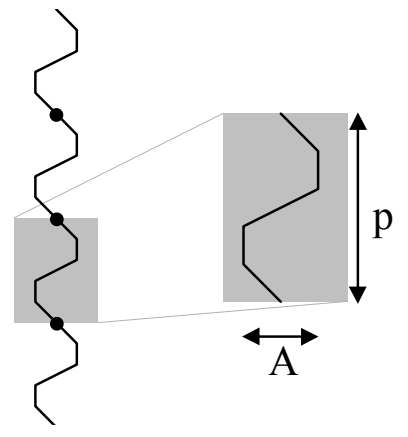

\begin{abstract}
Abb. 10.9 Definition der Stufentopologie durch aneinandergefügte Elementarzellen der Periodenlänge p und lateralen ,peak-to-peak““Modulationstiefe A. Wenn nicht anders angegeben, werden für die Simulation sinusförmige Elementarzellen verwendet.
\end{abstract}

Jede Elementarzelle setzt sich aus 100 hintereinander angeordneten Streuzentren zusammen, deren Abstände untereinander deutlich kleiner als die Wellenlänge gewählt sind. Die Anordnung der Elementarzellen geht über den simulierten Bildbereich so weit hinaus (typischerweise der fünf- bis zehnfache Wert der Simulationsfeldabmessungen), dass Beugungseffekte an den Rändern des Gitters vernachlässigt werden können.

Zusätzlich zur LDOS wird auch die Ableitung der LDOS nach der Energie berechnet, indem die LDOS jeweils einzeln für zwei sehr nahe beieinander liegenden Energien berechnet und dann die Differenz der Muster gebildet wird. Das Ergebnis dieser Operation gibt in erster Näherung eine differentielle LDOS, also ein Maß für die Thermospannung an.

Ein exemplarisches Beispiel für die Simulation eines Beugungsmusters ist in Abb. 10.10 dargestellt. Die reflektierende Stufe befindet sich in allen Simulationen auf der linken Seite. Die Interferenzmuster von der LDOS, Abb. 10.10 (a), und der Energieableitung der LDOS, Abb. 10.10 (b), ähneln sich sehr, wenn man von der Abstandsabhängigkeit des Kontrastes absieht. Es bietet sich deshalb an, zu einer alternativen grafischen Darstellungsart überzugehen, die den relativen Kontrast benachbarter Oszillationen unabhängig vom Abstand zur Stufe darstellt. Dies geschieht durch das „Herausrechnen“ der Einhüllendenfunktion, indem die LDOS der modulierten Stufe durch die Einhüllendenfunktion der geraden Stufe dividiert wird, wie in Abb. 10.10 (c) gezeigt. Diese Darstellung wird im folgenden „normierte“ LDOS genannt. In einem weiteren Schritt wird zeilenweise der Kontrast (d.h. die Einhüllende) der normierten LDOS berechnet ${ }^{32}$, Abb. 10.10 (d)). In dieser grafischen Darstellung sind helle Bereiche gleichbedeutend mit einer hohen Amplitude im Interferenzmuster bzw. dunkle Bereichen gleichbedeutend mit einer niedrigen Amplitude.

\footnotetext{
${ }^{32}$ Dies geschieht nach dem in Kap. 8.4 angegebenen Verfahren durch Bildung der Einhüllenden für die „normierte“ LDOS.
} 


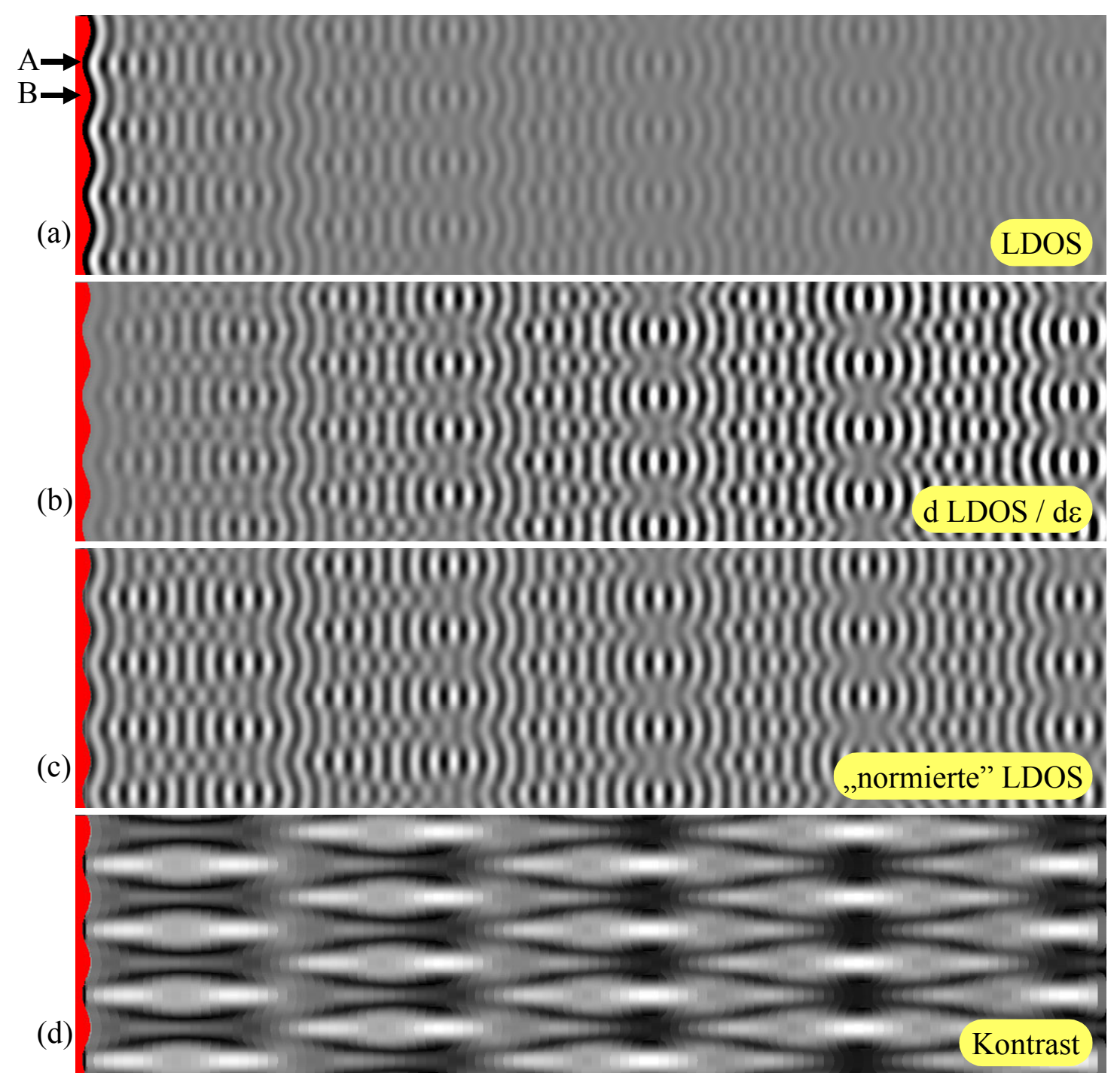

Abb. 10.10 Simulation des Interferenzmusters $(100 \mathrm{~nm} \times 25 \mathrm{~nm})$ an einer sinusförmig modulierten Stufe auf der linken Seite $(\mathrm{p}=6.3 \mathrm{~nm}, \mathrm{~A}=0.8 \mathrm{~nm})$. Dargestellt ist die LDOS in $(\mathrm{a})$, die Energieableitung der LDOS in (b), eine bezüglich der Einhüllendenfunktion für eine gerade Stufe ,,normierte“ LDOS in (c) (s. Text), sowie in (d) die Darstellung des relativen Kontrastes als Einhüllende von (c). Es sind deutlich periodisch angeordnete, rautenförmige Gebiete hoher Amplitudenstärke im Interferenzmuster zu erkennen, welche korreliert sind mit der periodischen lateralen Modulation der Stufe.

\subsubsection{Beschreibung des Beugungsmusters}

Die Simulation zeigt ein zweidimensionales „Schwebungsmuster“, d.h. es existieren nebenund untereinander angeordnete, rautenförmige Bereiche mit abwechselnd hohem und niedrigem Kontrast. Parallel zur Stufe besitzt das Muster dieselbe Periodenlänge $\mathrm{p}$ wie die Stufe selbst. Dies ist auch zu erwarten, da die Stufe bei einer Translation um $\mathrm{p}$ auf sich selbst abgebildet wird, und damit auch das Interferenzmuster dieselbe Translationssymmetrie besitzen muss.

In unmittelbarer Nähe zur Stufe ist ein Bereich mit hohem Kontrast immer gekoppelt an eine konvexe „Auswölbung“ der Stufe. 
Der „Schwebungs-Charakter“ des Beugungsmusters ist in Abb. 10.11 verdeutlicht, wo jeweils zwei um p/2 versetzte Querschnitte durch die LDOS dargestellt sind. In Bereichen mit hohem Kontrast ist die Amplitude etwa zweimal so hoch wie die einer perfekt geraden Stufe.

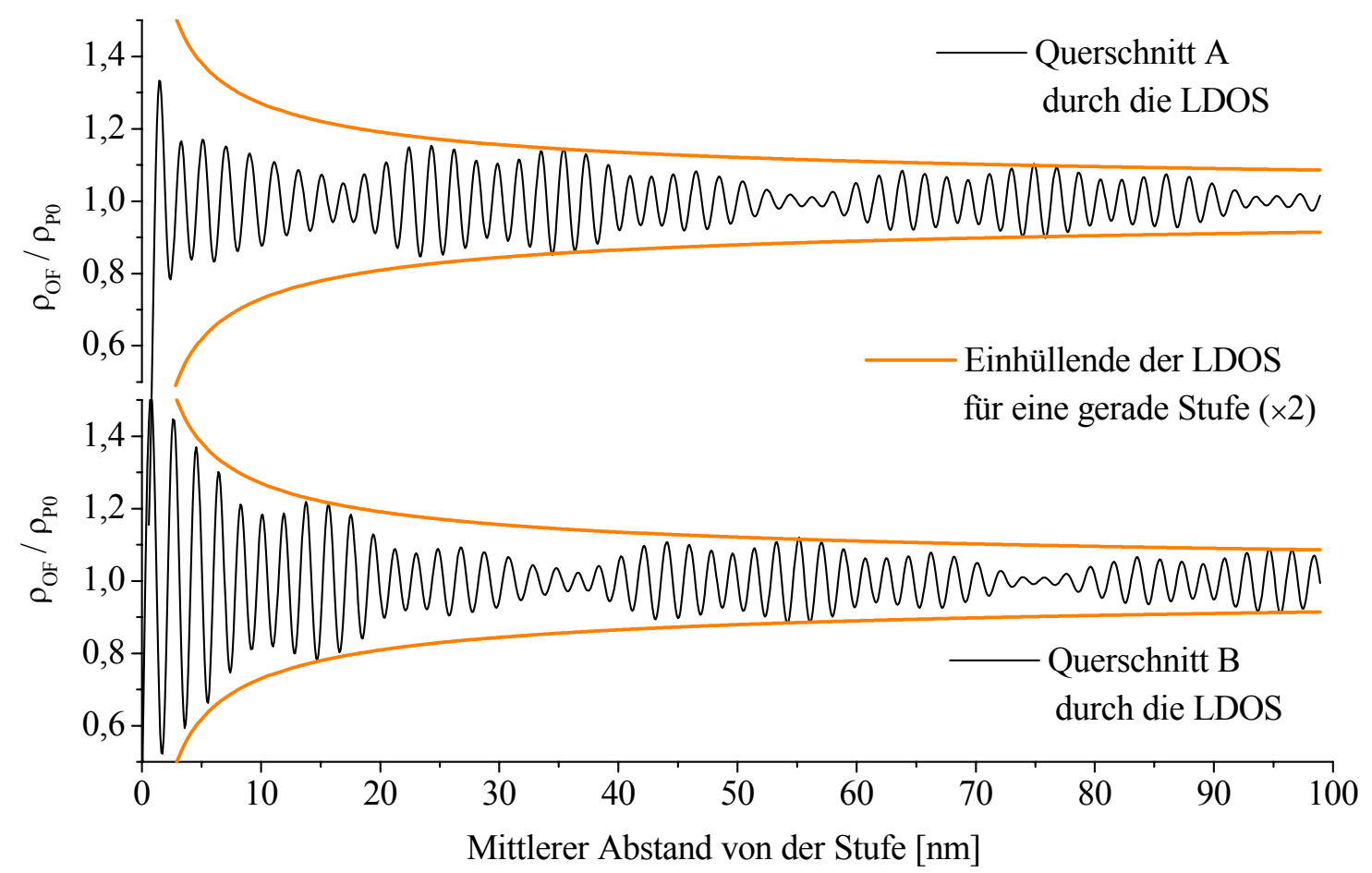

Abb. 10.11 Querschnitte durch die LDOS in Abb. 10.10 (a) (dort mit „A“ und „B“ markiert) im Vergleich zu der Einhüllenden für eine gerade Stufe (die Einhüllende ist mit einen Faktor 2 multipliziert, um die Existenz von Schwebungsmaxima zu verdeutlichen)

Zur Charakterisierung des Abklingverhaltens ist auch die Einhüllende für eine perfekt gerade Stufe mit eingezeichnet. Die Abbildung zeigt, dass grundsätzlich das Abklingverhalten der Oszillationen identisch zu dem einer perfekt geraden Stufe ist, d.h. die LDOS-Oszillationen werden mit $1 / \sqrt{\pi \mathrm{k}_{\mathrm{F}} \mathrm{x}}$ gedämpft. Dies ist nicht überraschend, da die Dämpfung der LDOS aus der Überlagerung verschiedener Wellenlängen resultiert (s. Kap. 7.1) und dieser Effekt weitestgehend unabhängig von der topologischen Form der Stufe ist.

$\mathrm{Zu}$ einem äquivalenten Ergebnis führt die (hier nicht dargestellte) Analyse der Energieableitung der LDOS; wie theoretisch erwartet (s. Kap. 8.2.3 steigt die Amplitude wie $\sqrt{\pi \mathrm{kx}}$ mit dem Abstand an.

\subsubsection{Variation der Stufenparameter}

Wenn die laterale Modulationstiefe der Stufe klein gegenüber der Wellenlänge der streuenden Elektronen ist, wird ein Schwebungsmuster gefunden, welches aus über- und nebeneinander angeordneten, rautenähnlichen Bereichen mit unterschiedlichem Kontrast besteht. 
Um systematisch zu untersuchen, welche Wirkung die modulierte Stufe auf dieses Muster hat, stehen folgende Variations-Parameter zur Verfügung:

- die topologische Form der Stufe,

- die laterale Modulationstiefe A der Stufe und

- die Periodizitätslänge $\mathrm{p}$ der Stufe.

In Abb. 10.12 wird die Periodizitätslänge $\mathrm{p}$ und in Abb. 10.13 die laterale peak-to-peak Modulationstiefe A der Stufe variiert.
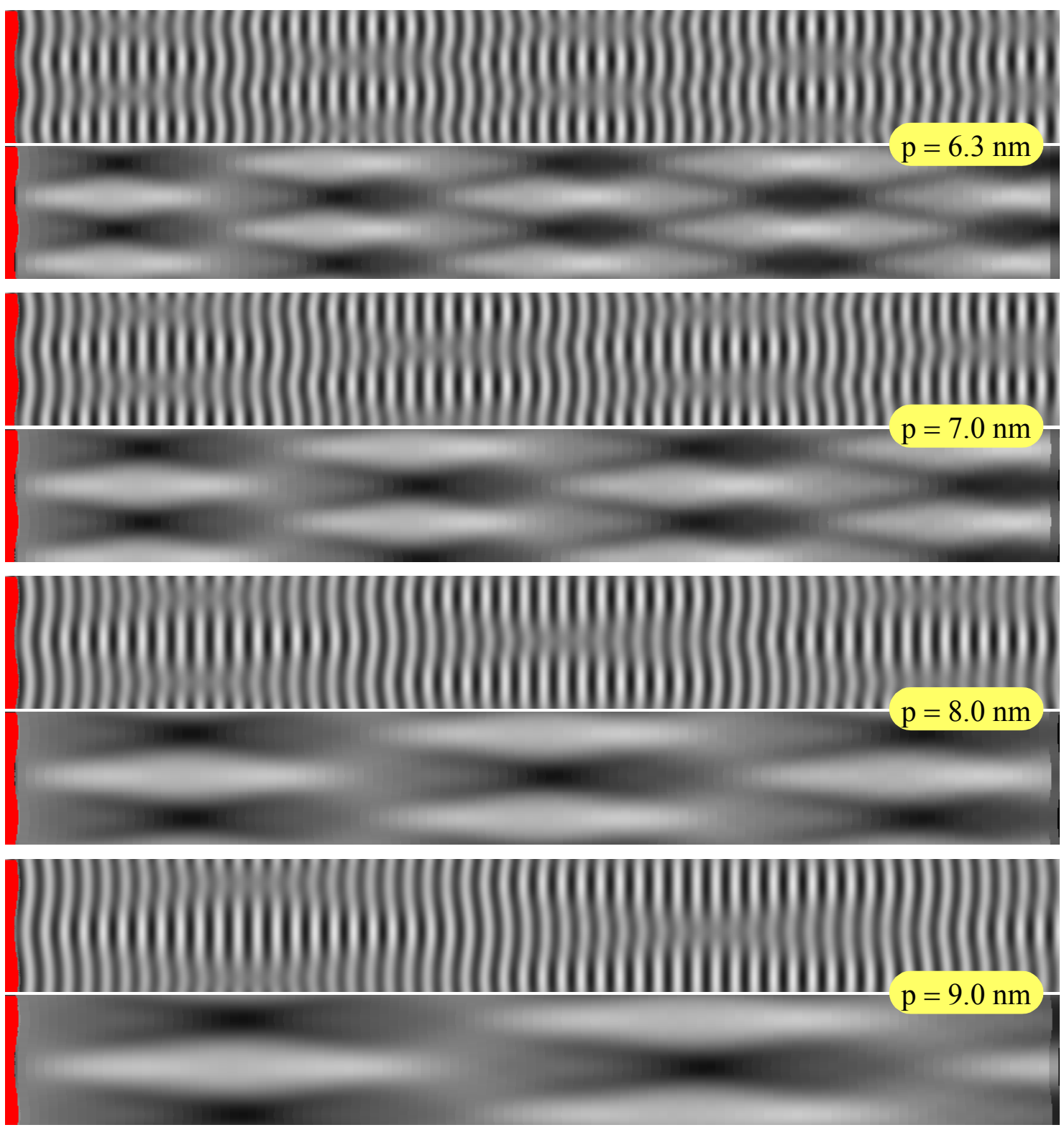

Abb. 10.12 Änderung des Interferenzmusters unter Variation der Periodenlänge p der Stufe. Die p-p Modulationstiefe der Stufe ist konstant A=0.2 nm. Dargestellt sind jeweils Paare von „normierter“ LDOS (oben) mit zugehörigem Kontrast (darunter). 
Die Ergebnisse für $\mathrm{A} \ll \lambda / 2 \ll p$ zeigen, dass eine Vergrößerung von $\mathrm{p}$ eine deutliche Ausdehnung der rautenförmigen Gebiete senkrecht zur Stufe mit sich bringt. Zudem entsteht in einem charakteristischen Abstand $d=2 p^{2} / \lambda_{F}$ zur Stufe ein Abbild der Stufentopologie im Interferenzmuster. In der Fresnel-Beugungstheorie ist dieses Phänomen als Talbot-Effekt bekannt [Pat, Tal]; die Talbotlänge d wurde erstmals von Lord Rayleigh berechnet [Ray]. Im Gegensatz dazu bewirkt eine Variation von A keine Größenänderung der rautenförmigen Gebiete. Innerhalb einer „Raute“ ändern sich jedoch die Details des Musters; mit zunehmender Modulationstiefe A tritt immer stärker ein zweites Schwebungsmuster hervor.

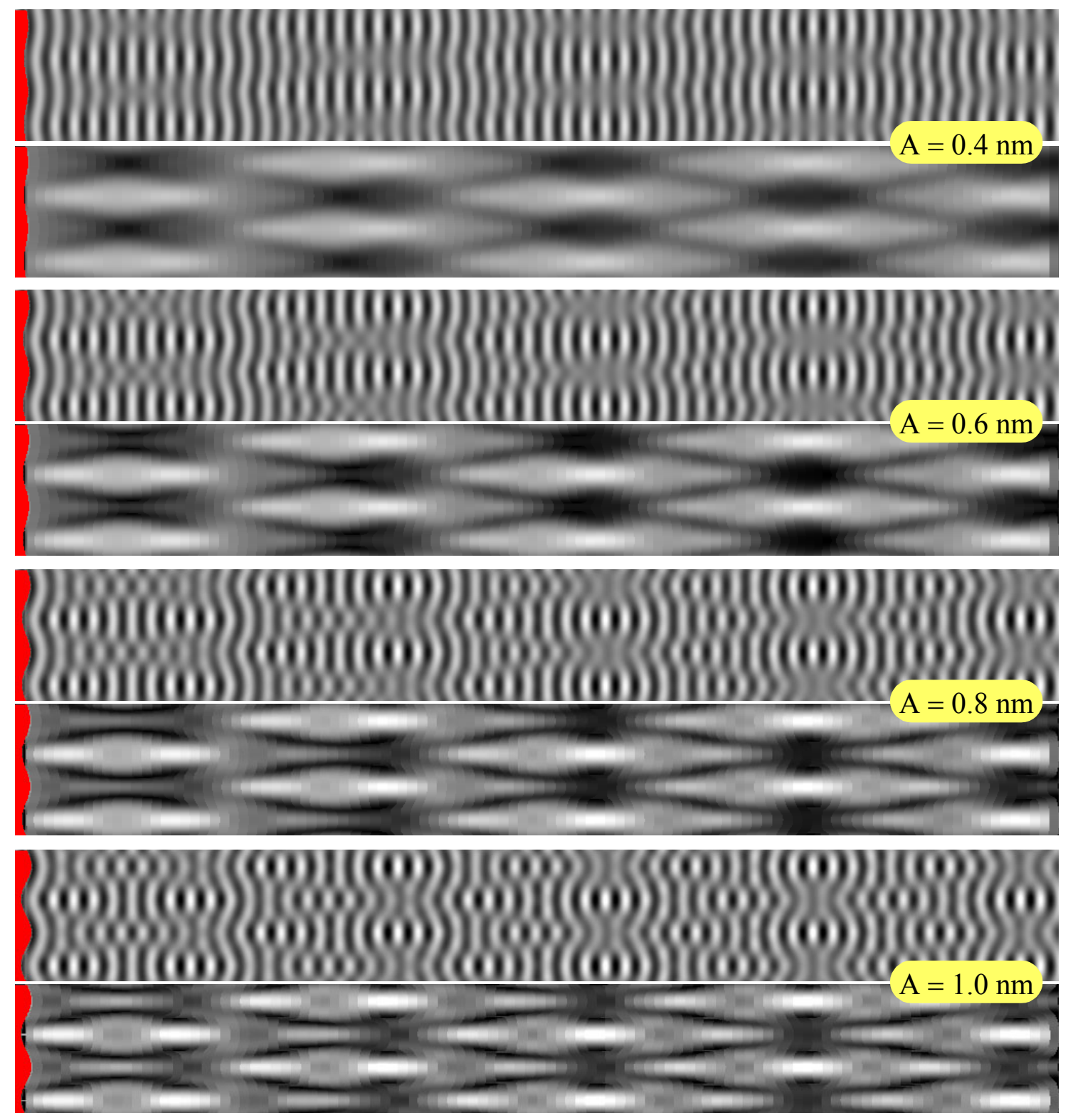

Abb. 10.13 Änderung des Interferenzmusters unter Variation der p-p Modulationstiefe A der Stufe. Die Periodenlänge der Stufe ist konstant $p=6.3 \mathrm{~nm}$ (diese Periodenlänge entsteht, wenn die Rekonstruktionslinien senkrecht zur Stufe verlaufen). Dargestellt sind jeweils Paare von „normierter“ LDOS (oben) mit zugehörigem Kontrast (darunter). 
Wenn sowohl A wie auch p sich der halben Wellenlänge $\lambda$ der streuenden Elektronenwellen nähern, dann entstehen komplexere Interferenzstrukturen. Auf diese sei hier nicht näher eingegangen, da die hier experimentell beobachteten Größen im Bereich $\mathrm{A}<1.0 \mathrm{~nm}$ und $\mathrm{p}>6.3 \mathrm{~nm}$ bei $\lambda / 2 \approx 1.8 \mathrm{~nm}$ liegen.

Wenn die topologische Form der Stufe geändert wird, wird ein ähnliches Ergebnis wie bei einer Variation von A gefunden, wie in Abb. 10.14 gezeigt ist.
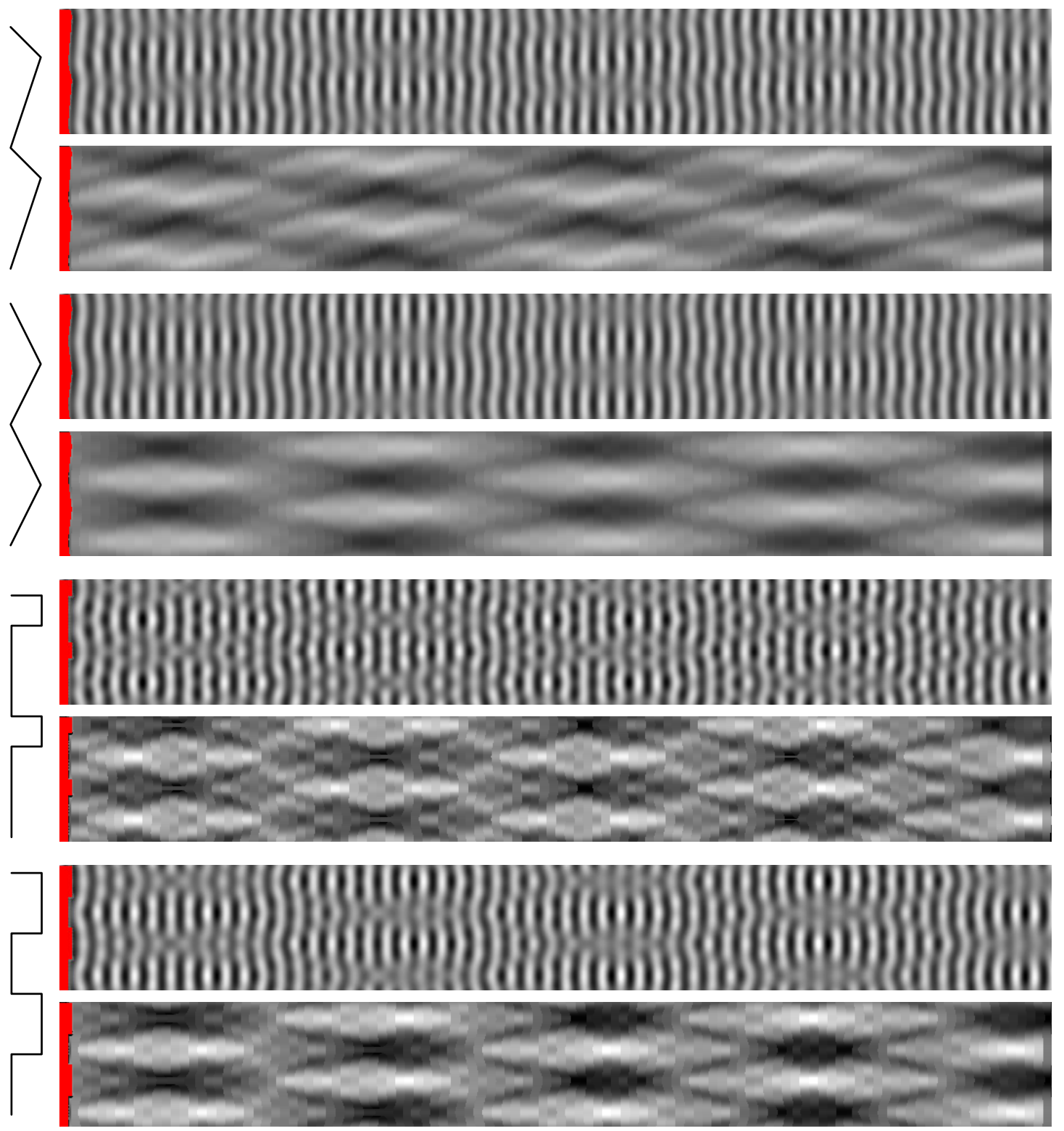

Abb. 10.14 Interferenzmuster unter Variation der Topologie der Stufe (am linken Rand ist jeweils eine Schemazeichnung der verwendeten Stufenform dargestellt). Die Periodenlänge der Stufe ist konstant $\mathrm{p}=6.3 \mathrm{~nm}$, die p-p Modulationstiefe ist konstant $\mathrm{A}=0.4 \mathrm{~nm}$.

Es ändert sich auch hier nicht die Länge oder Breite der Rauten. Das Interferenzmuster innerhalb einer Raute ist jedoch sehr empfindlich von der Detailtopologie der Stufe abhängig. 
In einer Zusammenfassung gesagt, besteht das Interferenzmuster aus einem zweidimensionalen „Schwebungsgitter“ mit Bereichen hohen und niedrigen Kontrastes. Die Größe dieser Bereiche ist durch die Periodenlänge p der modulierten Stufe bestimmt, während der Kontrast und die Detailstruktur von der Modulationstiefe A bzw. der topologischen Form der Stufe abhängig ist.

In der Nähe der Stufe wird immer bei einer konvexen „Auswölbung“ der Stufe ein Bereich mit hohem Kontrast gefunden. 


\subsection{Vergleich und Neuinterpretation experimenteller Daten}

Das simulierte Interferenzmusters kann mit den experimentellen Ergebnissen in der Nähe der Stufe innerhalb der ersten 10-20 Interferenzzyklen verglichen werden. Dies wird für die in Abb. 10.7 vorgestellte Messung durchgeführt, indem in der Simulation eine sinusförmige Stufe mit Periodenlänge $p=7.0 \mathrm{~nm}$ und $\mathrm{p}$-p Modulationstiefe $\mathrm{A}=0.8 \mathrm{~nm}$ moduliert wird. Die simulierten Daten werden ferner mit der analytisch erhaltenen Einhüllenden multipliziert, um den Effekt der thermischen Dämpfung zu berücksichtigen. Das Ergebnis ist in Abb. 10.15 gezeigt.

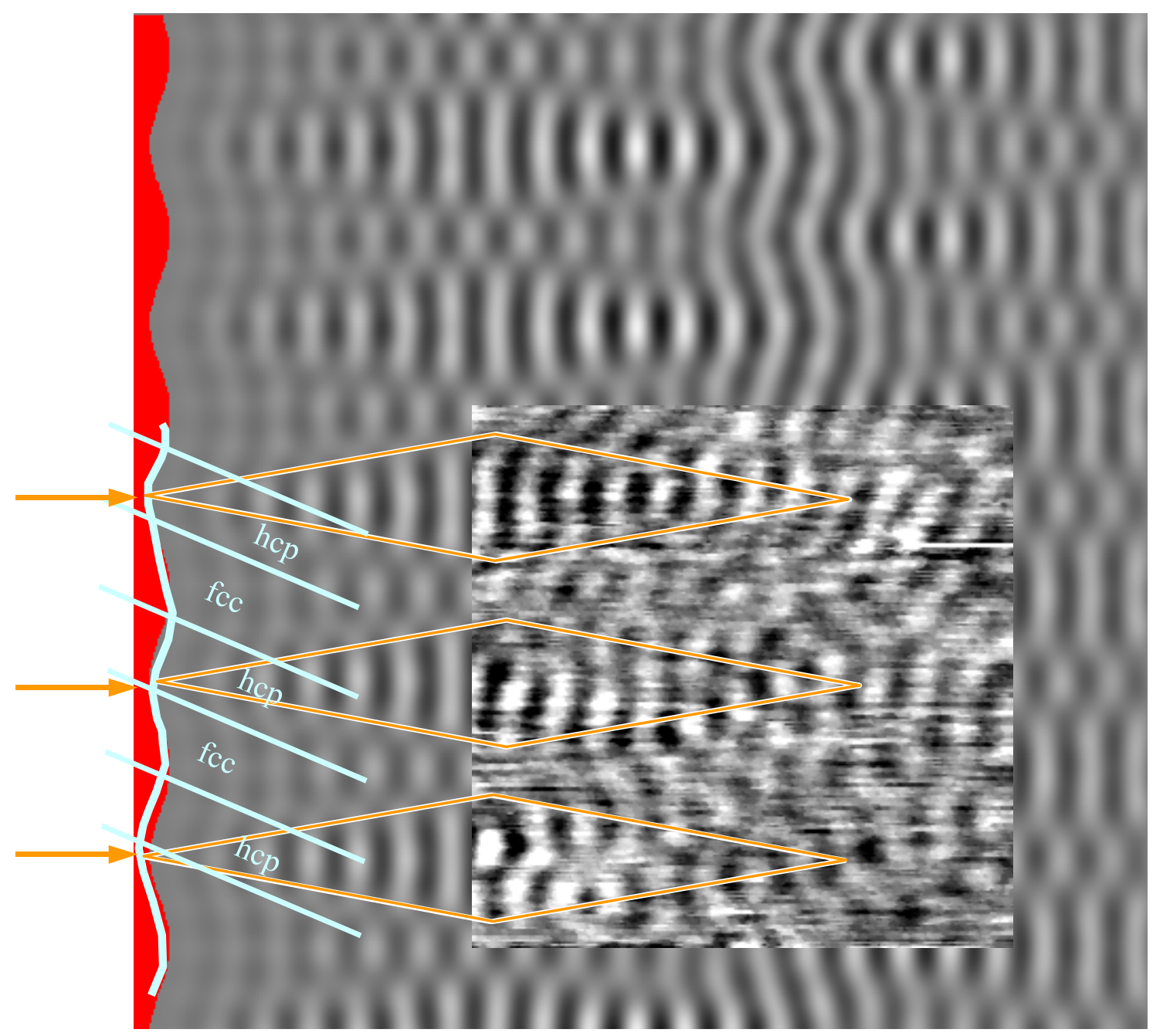

Abb. 10.15 Vergleich von experimentellen mit simulierten Daten. Im Hintergrund die simulierte Thermospannung zu der in Abb. 10.7 vorgestellten experimentellen Messung. Die Kontur der realen Stufe ist als Linienzug eingezeichnet. Die für die Simulation nachmodellierte Stufe ist sinusförmig mit $\mathrm{p}=7.0 \mathrm{~nm}$ und $\mathrm{A}=0.8 \mathrm{~nm}$. Ebenfalls eingezeichnet ist der Verlauf der experimentell sichtbaren Rekonstruktionslinien ( ) und die Zuordnung der hcp- und fcc- Rekonstruktions-Domänen. Den konvexen Auswölbungen der Stufen $(\rightarrow$ ) sind rautenförmige Gebiete mit starkem Kontrast $(\longrightarrow$ ) im Interferenzmuster zugeordnet. 
Sowohl experimentelle wie auch simulierte Daten zeigen eine Übereinstimmung bezüglich den Gebieten mit hohem Kontrast. Diese sind jeweils den drei in Abb. 10.15 markierten „Rauten“ zuzuordnen, welche wiederum mit drei topografischen konvexen Auswölbungen der Stufe korreliert sind.

Die periodische Modulation der Stufe durch die Rekonstruktion wirkt also als Beugungsgitter für Elektronenwellen.

Die Schwebungserscheinungen im Interferenzmuster geben eine fundierte Neuinterpretation für die bereits im Abschnitt 10.2 vorgestellte Beobachtung von D. Fujita [Fuj 1], wonach eine unterschiedliche Amplitude der stehenden Wellen innerhalb der hcp- und der fcc-Region gefunden wird.

An Orten, wo die hcp-Region der Rekonstruktion die Stufe überquert, wird eine konvexe Auswölbung der Stufe beobachtet (s. Abb. 10.6 und 10.15. An diesen Stellen wird gemäß den Simulationsergebnissen immer ein erhöhter Kontrast im Beugungsmuster gefunden.

Für den Fall, dass die Rekonstruktionslinien senkrecht zur Stufe stehen, wird also innerhalb der hcp-Region ein erhöhter Kontrast gefunden.

Dies führt zu folgenden Schlussfolgerungen:

- Die Elektronen der Oberflächenzustände bei $\mathrm{E}_{\mathrm{F}}$ werden nicht an der Rekonstruktion gestreut und erfahren auch kein Confinement im Rekonstruktionspotenzial.

- Die beobachteten Unterschiede der Interferenzamplituden in den hcp- und fcc-Regionen sind ein Beugungseffekt der Elektronenwellen an der Stufe.

Die Abkehr von der ursprünglichen Annahme, dass die Elektronenwellen am Rekonstruktionspotenzial gestreut werden [Fuj 1], wird auch unterstützt von den spektroskopischen Daten, welche von W. Chen [Che 1] und L. Bürgi [Bür 1] vorgestellt wurden. Nach der Interpretation dieser spektroskopischen Daten besitzen die Oberflächenzustände auf einer ungestörten Oberfläche eine gleichmäßige, nicht mit der Rekonstruktion korrelierte Ladungsdichte. 


\section{Zusammenfassung}

Der thermoelektrische Effekt am Tunnelkontakt eines STM wird dazu benutzt, die Thermospannung zwischen geheizter Spitze und kühlerer Probe mit einer nm-Auflösung zu kartieren. Die Thermospannung ist sehr empfindlich von der differentiellen lokalen Zustandsdichte der Probe abhängig.

Auf $\mathrm{Au}(111)$-Oberflächen werden die Interferenzmuster von Elektronenwellen der Oberflächenzustände untersucht. Die Oberflächenzustände auf $\mathrm{Au}(111)$ bilden ein zweidimensionales Elektronengas, in dem die Elektronen in Form von ebenen Wellen mit einer nahezu isotropen, parabolischen Dispersionsrelation entlang der Oberfläche propagieren können. Bei der Reflexion von Elektronenwellen an Streuern werden Interferenzeffekte in Form eines stehenden Wellenfeldes in der Thermospannung sichtbar.

Das Interferenzmuster in der Thermospannung ist in dieser Arbeit erstmals bei tiefen Temperaturen von $80 \mathrm{~K}$ kartiert. An monatomaren Stufen wird das Interferenzmuster gegenüber Messungen bei Raumtemperatur auch in deutlich größeren Abständen bis zu $30 \mathrm{~nm}$ (ca. 15 Oszillationszyklen) von der Stufe entfernt eindeutig identifiziert. Dabei zeigt die Einhüllende der stehenden Wellen in der Thermospannung einen charakteristischen Verlauf: Die Amplitude der stehenden Welle steigt mit dem Abstand von der Stufe zunächst an, bis sie nach Erreichen eines Maximums bei ca. 10-15 nm exponentiell abklingt. Mit alternativen Verfahren (Topografie oder dI/dU-Spektroskopie) wird stets eine mit dem Abstand von der Stufe abklingende stehende Welle gefunden.

Im Rahmen dieser Arbeit ist es erstmals gelungen, die stehenden Wellen in der Thermospannung analytisch zu beschreiben. Wenn $\mathrm{T}_{\min }$ und $\mathrm{T}_{\max }$ die jeweils tiefste bzw. höchste Temperatur des Systems Spitze/Probe darstellen, dann verbreitert sich der Bereich, innerhalb dessen die Thermospannungsamplitude mit dem Abstand von der Stufe zunimmt, proportional zu $1 / \mathrm{T}_{\max }$. Das Abklingverhalten der stehenden Wellen wird zum einen durch einen Term $\exp \left(-\right.$ const. $\left.\cdot \mathrm{T}_{\min } \cdot \mathrm{x}\right)$ beschrieben. Zum anderen führt auch Elektron-PhononStreuung zu einer endlichen Kohärenzlänge $\mathrm{L}_{\varphi}$, welche durch quantitative Auswertung der experimentell gemessenen Thermospannungsamplituden bestimmt werden kann. Es ist gelungen, die Kohärenzlänge bzw. Lebensdauer der Oberflächenzustände bei der Fermienergie zu bestimmen. Für $300 \mathrm{~K}$ ergibt sich eine beste Übereinstimmung mit experimentellen Werten bei $\mathrm{L}_{\varphi}=30 \mathrm{~nm}$ bzw. $\tau_{\varphi}=38 \mathrm{fs}$, wobei als neue Untergrenze $\mathrm{L}_{\varphi}>10 \mathrm{~nm}$ bzw. $\tau_{\varphi}>13 \mathrm{fs}$ angegeben werden kann. Für $80 \mathrm{~K}$ ergibt sich eine beste Übereinstimmung mit experimentellen Werten bei $\mathrm{L}_{\varphi}=60 \mathrm{~nm}$ bzw. $\tau_{\varphi}=76 \mathrm{fs}$, wobei als neue Untergrenze $\mathrm{L}_{\varphi}>40 \mathrm{~nm}$ bzw. $\tau_{\varphi}>51$ fs angegeben werden kann.

In einem weiteren Themenbereich dieser Arbeit kann gezeigt werden, dass die Thermospannung am Tunnelkontakt auch direkt in der Topografie Auswirkungen zeigt. Wenn 
die Thermospannung betragsmäßig die Größenordnung der Tunnelvorspannung bei alleinigen Topografiemessungen erreicht, wird die topografische Korrugation deutlich gesteigert. Experimentell wird bei Temperaturen um $80 \mathrm{~K}$ ein Interferenzmuster in der Topografie gefunden, dessen Korrugationsamplitude deutlich über der für Temperaturgleichheit theoretisch erwarteten liegt. Dieser Effekt hat Auswirkungen auf die Interpretation von Daten, wie sie beispielsweise mit temperaturvariablen STM erhalten werden, soweit ein Temperaturunterschied zwischen Spitze und Probe besteht.

Im letzten Themenbereich dieser Arbeit wird der Einfluss der Au(111)-HerringboneRekonstruktion auf das Interferenzmuster an monatomaren Stufen untersucht. Es werden Beispiele gefunden, in denen monatomare Stufen eine periodische Modulation durch die Rekonstruktion erfahren und als Beugungsgitter für Elektronenwellen wirken. Das experimentell gefundene Beugungsmuster stimmt mit theoretisch simulierten Mustern überein.

Die Untersuchung von Interferenzmustern in Bereichen bis zu $30 \mathrm{~nm}$ Abstand von der Stufe ergibt, dass der Verlauf der stehenden Wellen nicht mit den quasi-eindimensionalen Domänen der Herringbone-Rekonstruktion korreliert ist. Damit wird zumindest für Elektronen nahe der Fermienergie ein Modell widerlegt, wonach die Rekonstruktionsdomänen als ConfinementPotenzial für Oberflächenzustände wirken. Diese Modellannahme basiert auf topografischen Beobachtungen, bei denen nahe der Stufenkante in der hcp-Domäne der Rekonstruktion eine höhere Amplitude in den stehenden Wellen gefunden wird als in der fcc-Domäne. Demgegenüber kann dieser Effekt auch im Modell einer Stufe, welche als Beugungsgitter wirkt, erklärt werden. 


\section{Anhang A: Lokale Zustandsdichte an geradlinigen Stufen}

In diesem Abschnitt wird die LDOS an einer geradlinigen Stufe für beliebige AmplitudenReflexionsgrade R und Phasenverschiebungen $\varphi$ bei der Reflexion von Elektronenwellen an der Stufe berechnet. Die Bezeichnungen sind aus Kapitel 7.1 übernommen. Es wird im folgenden angenommen, dass $\mathrm{R}, \varphi$ und $\psi(\mathrm{z})$ unabhängig von der Richtung $\overrightarrow{\mathrm{k}}_{\|}$der Elektronenwelle sind. Die folgenden Formeln beziehen sich nur auf die positive Halbraumhälfte $x>0$. Die auf die Stufe einfallende Welle lässt sich zeitabhängig mit einer Winkelfrequenz $\omega$ ausdrücken zu

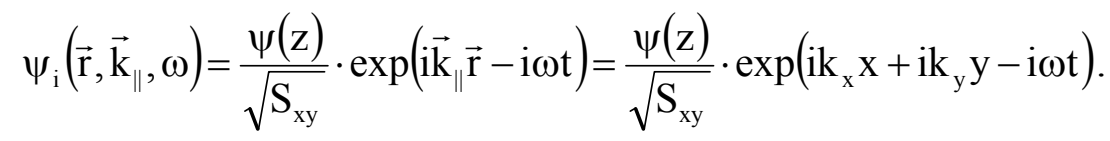

Die reflektierte Welle besitzt die Form

$$
\psi_{\mathrm{r}}\left(\overrightarrow{\mathrm{r}}, \overrightarrow{\mathrm{k}}_{\|}, \omega\right)=\mathrm{R} \cdot \frac{\psi(\mathrm{z})}{\sqrt{\mathrm{S}_{\mathrm{xy}}}} \cdot \exp \left(-\mathrm{ik}_{\mathrm{x}} \mathrm{x}+\mathrm{ik}_{\mathrm{y}} \mathrm{y}-\mathrm{i} \omega \mathrm{t}+\mathrm{i} \varphi\right) .
$$

Die Überlagerung von einfallender und reflektierter Welle ergibt

$$
\psi\left(\overrightarrow{\mathrm{r}}, \overrightarrow{\mathrm{k}}_{\|}, \omega\right)=\frac{\psi(\mathrm{z})}{\sqrt{\mathrm{S}_{\mathrm{xy}}}} \cdot \exp \left(\mathrm{ik}_{\mathrm{y}} \mathrm{y}+\mathrm{i} \omega \mathrm{t}\right) \cdot\left[\exp \left(\mathrm{ik}_{\mathrm{x}} \mathrm{x}\right)+\mathrm{R} \cdot \exp \left(-\mathrm{ik}_{\mathrm{x}} \mathrm{x}+\mathrm{i} \varphi\right)\right]
$$

Die Bildung des Betragsquadrates kann mittels geometrischer Vektoraddition [Bro, Kap. 2.6.3.1.2] berechnet werden:

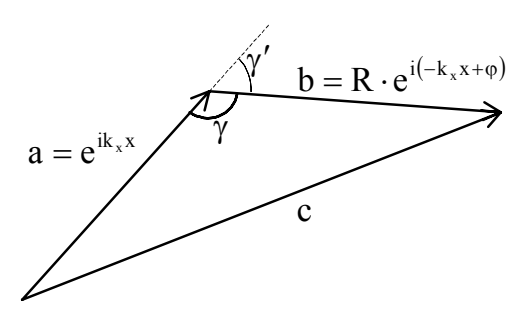

$$
\begin{aligned}
& \text { Abb. A.1 Berechnung des Betragsquadrates der Wellenüberla- } \\
& \text { gerung. Es gilt die Beziehung }|c|^{2}=|a|^{2}+|b|^{2}-2 \cdot|a| \cdot|b| \cdot \cos (\gamma) \text {. } \\
& \text { Mit } \gamma=\pi-\gamma^{\prime}=\pi+2 k_{x} x-\varphi \text { folgt } \\
& |c|^{2}=1+R^{2}+2 \cdot R \cdot \cos \left(-2 k_{x} x+\varphi\right)
\end{aligned}
$$

$$
\left|\psi\left(\overrightarrow{\mathrm{r}}, \overrightarrow{\mathrm{k}}_{\|}\right)\right|^{2}=\frac{|\psi(\mathrm{z})|^{2}}{\mathrm{~S}_{\mathrm{xy}}} \cdot\left[1+\mathrm{R}^{2}+2 \cdot \mathrm{R} \cdot \cos \left(-2 \mathrm{k}_{\mathrm{x}} \mathrm{x}+\varphi\right)\right] .
$$

Anschaulich gedeutet enthält der letzte Term die Betragsquadrate der auf die Stufe zulaufenden Welle (Summand 1), der reflektierten Welle (Summand $\mathrm{R}^{2}$ ) sowie eine stehende Welle als Interferenzterm (Kosinusterm). Es ist jedoch noch nicht der Anteil der Elektronen berücksichtigt, welche von der negativen Halbseite $\mathrm{x}<0$ aus über die Stufe hinweg transmittieren bzw. an der Stufe aus den Volumenzuständen heraus in die 
Oberflächenzustände hineingestreut werden. Dieser Anteil liefert einen nicht interferierenden, konstanten Hintergrundbeitrag. Da in die Oberflächenzustände ebenso viele Elektronen hinein- wie hinausstreuen, ist das Betragsquadrat dieses Anteils gegeben zu

$$
\left|\psi\left(\overrightarrow{\mathrm{r}},-\overrightarrow{\mathrm{k}}_{\|}\right)\right|^{2}=\frac{|\psi(\mathrm{z})|^{2}}{\mathrm{~S}_{\mathrm{xy}}} \cdot\left(1-\mathrm{R}^{2}\right)
$$

Diese beiden Anteile können zu einer lokalen Zustandsdichte $\rho_{\overrightarrow{\mathrm{k}}}(\overrightarrow{\mathrm{r}}, \mathrm{E})$ addiert werden:

$$
\rho_{ \pm \overrightarrow{\mathrm{k}}_{\|}}(\overrightarrow{\mathrm{r}}, \mathrm{E})=\left|\psi\left(\overrightarrow{\mathrm{r}}, \overrightarrow{\mathrm{k}}_{\|}\right)\right|^{2}+\left|\psi\left(\overrightarrow{\mathrm{r}},-\overrightarrow{\mathrm{k}}_{\|}\right)\right|^{2}=\frac{|\psi(\mathrm{z})|^{2}}{\mathrm{~S}_{\mathrm{xy}}} \cdot 2 \cdot\left[1+\mathrm{R} \cdot \cos \left(-2 \mathrm{k}_{\mathrm{x}} \mathrm{x}+\varphi\right)\right] .
$$

Die lokale Zustandsdichte $\rho_{\mathrm{OF}}(\overrightarrow{\mathrm{r}}, \mathrm{E})$ berechnet sich aus der Summe der Betragsquadrate aller zu einer Energie E gehörenden Wellenfunktionen. Für die parabolische, isotrope Dispersionsrelation von $\mathrm{Au}(111)$ bedeutet dies, dass alle Wellenfunktionen mit konstantem Wellenvektorbetrag $\mathrm{k}_{\|}$aufsummiert werden. Die Dichte der Zustände eines Zweidimensionalen Elektronengases ist $\mathrm{m}^{*} /\left(\pi \hbar^{2}\right)$ [Iba, Kap. 12.7]. Für einen Wellenvektor, welcher mit der $y$-Achse einen Winkel $\alpha$ einschließt, folgt $k_{x}=k_{\|} \cdot \sin (\alpha)$. Die Summe für die LDOS, Gleichung (2.2), kann in ein Integral umgewandelt werden:

$$
\begin{aligned}
& \rho_{\mathrm{OF}}(\overrightarrow{\mathrm{r}}, \mathrm{E})=\sum_{\overrightarrow{\mathrm{k}}_{\|}}\left|\psi\left(\overrightarrow{\mathrm{r}}, \overrightarrow{\mathrm{k}}_{\|}\right)\right|^{2} \cdot \delta\left(E\left(\mathrm{k}_{\|}\right)-\mathrm{E}\right)=\frac{\mathrm{m}^{*}}{\pi \hbar^{2}} \cdot \mathrm{S}_{\mathrm{xy}} \cdot \int_{0}^{\pi} \rho_{ \pm \overrightarrow{\mathrm{k}_{\|}}}(\overrightarrow{\mathrm{r}}, \mathrm{E}) \cdot \frac{\mathrm{d} \alpha}{2 \pi} \\
& =\frac{\mathrm{m}^{*}}{\pi \hbar^{2}} \cdot|\psi(\mathrm{z})|^{2} \cdot\left(1+\frac{\mathrm{R}}{\pi} \cdot \int_{0}^{\pi} \cos \left(-2 \mathrm{k}_{\|} \mathrm{x} \cdot \sin (\alpha)+\varphi\right) \cdot \mathrm{d} \alpha\right) .
\end{aligned}
$$

Aus den Additionstheoremen trigonometrischer Funktionen [Bro, Kap. 2.5.2.1.3] lässt sich der Integralausdruck umformen zu

$$
\begin{aligned}
& \rho_{\mathrm{OF}}(\overrightarrow{\mathrm{r}}, \mathrm{E})=\frac{\mathrm{m}^{*}}{\pi \hbar^{2}} \cdot|\psi(\mathrm{z})|^{2} \\
& \cdot\left[1+\mathrm{R} \cdot\left(\frac{\cos (\varphi)}{\pi} \int_{0}^{\pi} \cos \left(2 \mathrm{k}_{\|} \mathrm{x} \cdot \sin (\alpha)\right) \cdot \mathrm{d} \alpha+\frac{\sin (\varphi)}{\pi} \int_{0}^{\pi} \sin \left(2 \mathrm{k}_{\|} \mathrm{x} \cdot \sin (\alpha)\right) \cdot \mathrm{d} \alpha\right)\right] .
\end{aligned}
$$

Das Integral mit der Kosinusfunktion ergibt nach [Bro, Kap. 3.3.1.3.4.]

$$
\frac{1}{\pi} \int_{0}^{\pi} \cos \left(2 \mathrm{k}_{\|} \mathrm{x} \cdot \sin (\alpha)\right) \cdot \mathrm{d} \alpha=\mathrm{J}_{0}\left(2 \mathrm{k}_{\|} \mathrm{x}\right)
$$

wobei $\mathrm{J}_{0}$ die Besselfunktion erster Gattung nullter Ordnung ist. Das Integral mit der Sinusfunktion ergibt nach [Gra, 3.715, 16] 
(A.10)

$$
\frac{1}{\pi} \int_{0}^{\pi} \sin \left(2 \mathrm{k}_{\|} \mathrm{x} \cdot \sin (\alpha)\right) \cdot \mathrm{d} \alpha=\mathrm{H}_{0}\left(2 \mathrm{k}_{\|} \mathrm{x}\right)
$$

wobei $\mathrm{H}_{0}$ die Struvefunktion nullter Ordnung ist. Die lokale Zustandsdichte der Oberflächenzustände beträgt also

$$
\rho_{\mathrm{OF}}(\overrightarrow{\mathrm{r}}, \mathrm{E})=\frac{\mathrm{m}^{*}}{\pi \hbar^{2}} \cdot|\psi(\mathrm{z})|^{2} \cdot\left(1+\mathrm{R} \cdot\left[\cos (\varphi) \cdot \mathrm{J}_{0}\left(2 \mathrm{k}_{\|} \mathrm{x}\right)+\sin (\varphi) \cdot \mathrm{H}_{0}\left(2 \mathrm{k}_{\|} \mathrm{x}\right)\right]\right)
$$

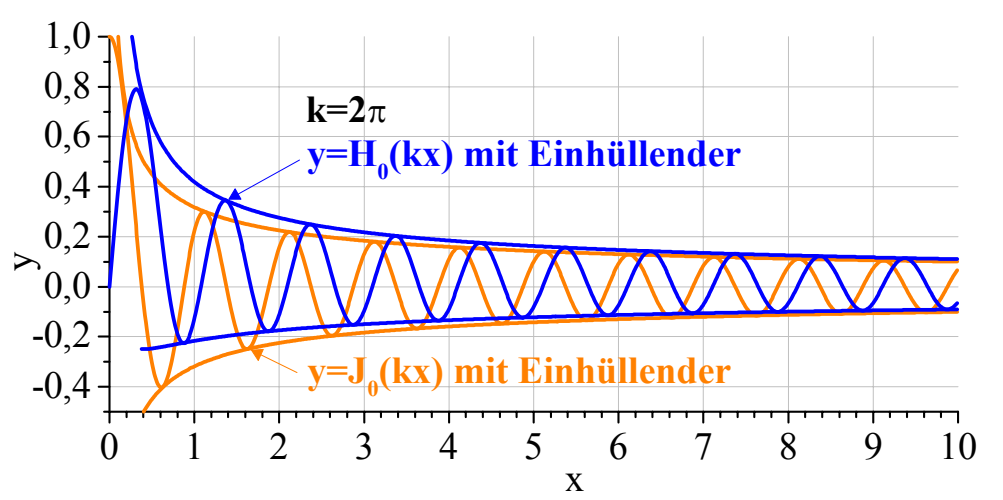

Abb. A.2 Darstellung der Funktionen $\mathrm{J}_{0}(\mathrm{kx})$ und $\mathrm{H}_{0}(\mathrm{kx})$ für $\mathrm{k}=2 \pi$.

Im Vergleich besitzen $\mathrm{J}_{0}$ und $\mathrm{H}_{0}$ ähnliche Eigenschaften: Im Grenzfall $\mathrm{x} \rightarrow \infty$ gelten die asymptotischen Näherungen [Wat]

$$
\mathrm{J}_{0}(\mathrm{x}) \rightarrow \sqrt{\frac{2}{\pi \mathrm{x}}} \cdot \cos \left(\mathrm{x}-\frac{\pi}{4}\right)
$$

$$
\mathrm{H}_{0}(\mathrm{x}) \rightarrow \frac{2}{\pi \mathrm{x}}+\sqrt{\frac{2}{\pi \mathrm{x}}} \cdot \sin \left(\mathrm{x}-\frac{\pi}{4}\right)
$$

deren Einhüllenden in Abb. A.2 mit eingezeichnet sind. Die Abweichungen der asymptotischen Näherungen von $\mathrm{J}_{0}$ bzw. $\mathrm{H}_{0}$ klingen proportional zu 1/x mit dem Abstand ab und betragen bereits nach der ersten Oszillationsperiode maximal nur noch $0.7 \%$ des Einhüllendenwertes.

Für große Abstände von der Stufe ergeben diese Näherungen eingesetzt in (A.11) und unter erneuter Anwendung trigonometrischer Additionstheoreme [Bro, Kap. 2.5.2.1.3]:

$$
\rho_{\mathrm{OF}}(\overrightarrow{\mathrm{r}}, \mathrm{E}) \rightarrow \frac{\mathrm{m}^{*}}{\pi \hbar^{2}} \cdot|\psi(\mathrm{z})|^{2} \cdot\left(1+\frac{\mathrm{R}}{\sqrt{\pi \mathrm{k}_{\|} \mathrm{x}}} \cdot \cos \left(2 \mathrm{k}_{\|} \mathrm{x}-\frac{\pi}{4}-\varphi\right)+\frac{\mathrm{R} \cdot \sin (\varphi)}{\pi \mathrm{k}_{\|} \mathrm{x}}\right) .
$$




\section{Anhang B: Analytische Herleitung der Oszillationseinhüllenden im Tersoff- Hamann Modell}

Es sei $\mathrm{U}_{\mathrm{Th}}(\mathrm{x})$ die Thermospannung als Funktion des Abstandes $\mathrm{x}$ von der Stufe, welche unter der Randbedingung eines verschwindenden Tunnelstromes $\mathrm{I}_{\mathrm{T}}=0$ gemessen wird. Die elektrochemischen Potenziale von Probe und Spitze unterscheiden sich um den Betrag $\mathrm{e} \cdot \mathrm{U}_{\mathrm{Th}}(\mathrm{x})$. Wenn die Abkürzung $\Delta \mu_{\mathrm{ec}}=\mathrm{e} \cdot \mathrm{U}_{\mathrm{Th}}(\mathrm{x}) / 2$ eingeführt wird $\varepsilon$ die Energiedifferenz relativ zum gemittelten elektrochemischen Potenzial von Probe und Spitze angibt, dann wird die Zustandsdichten der Spitze mit $\rho_{\mathrm{S}}\left(\varepsilon+\Delta \mu_{\mathrm{ec}}\right)$ und die lokale Zustandsdichte (LDOS) der Probe mit $\rho_{\mathrm{P}}\left(\overrightarrow{\mathrm{r}}, \varepsilon-\Delta \mu_{\mathrm{ec}}\right)$ beschrieben. Entsprechend ist $\mathrm{f}\left(\varepsilon+\Delta \mu_{\mathrm{ec}}, \mathrm{T}_{\mathrm{S}}\right)$ die Fermifunktion der Spitze mit Temperatur $\mathrm{T}_{\mathrm{S}}$ und $\mathrm{f}\left(\varepsilon-\Delta \mu_{\mathrm{ec}}, \mathrm{T}_{\mathrm{P}}\right)$ die Fermifunktion der Probe mit Temperatur $\mathrm{T}_{\mathrm{P}}$. Der Tunnelstrom wird dann im Tersoff-Hamann Modell beschrieben zu

$$
\mathrm{I}_{\mathrm{T}}=0=\int_{-\infty}^{\infty} \rho_{\mathrm{S}}\left(\varepsilon+\Delta \mu_{\mathrm{ec}}\right) \cdot \rho_{\mathrm{P}}\left(\overrightarrow{\mathrm{r}}, \varepsilon-\Delta \mu_{\mathrm{ec}}\right) \cdot\left(\mathrm{f}\left(\varepsilon+\Delta \mu_{\mathrm{ec}}, \mathrm{T}_{\mathrm{S}}\right)-\mathrm{f}\left(\varepsilon-\Delta \mu_{\mathrm{ec}}, \mathrm{T}_{\mathrm{P}}\right)\right) \cdot \mathrm{d} \varepsilon
$$

$\Delta \mu_{\mathrm{ec}}$ ist sehr klein gegenüber der thermischen Verbreiterung $\mathrm{k}_{\mathrm{B}} \mathrm{T}$ (experimentell wurde $\Delta \mu_{\mathrm{ec}} / \mathrm{k}_{\mathrm{B}} \mathrm{T} \approx 10^{-3}$ gemessen). Die relative Verschiebung der Zustandsdichten von Spitze und Probe darf deshalb vernachlässigt werden, so dass (B.1) vereinfacht werden kann zu

$$
\mathrm{I}_{\mathrm{T}}=0=\int_{-\infty}^{\infty} \rho_{\mathrm{S}}(\varepsilon) \cdot \rho_{\mathrm{P}}(\overrightarrow{\mathrm{r}}, \varepsilon) \cdot\left(\mathrm{f}\left(\varepsilon+\Delta \mu_{\mathrm{ec}}, \mathrm{T}_{\mathrm{S}}\right)-\mathrm{f}\left(\varepsilon-\Delta \mu_{\mathrm{ec}}, \mathrm{T}_{\mathrm{P}}\right)\right) \cdot \mathrm{d} \varepsilon
$$

Ziel dieses Abschnittes soll die Ermittelung der Lösung $\mathrm{U}_{\mathrm{Th}}(\mathrm{x})=2 \Delta \mu_{\mathrm{ec}} / \mathrm{e}$ sein, welche Gleichung (B.2) erfüllt.

Zur Lösung von (B.2) werden die einzelnen Funktionen so weit wie möglich ,symmetrisiert“, d.h. als eine Summe einer geraden Funktion $\mathrm{g}(\mathrm{x})=\mathrm{g}(-\mathrm{x})$ und einer ungeraden Funktion $\mathrm{u}(\mathrm{x})=-\mathrm{u}(-\mathrm{x})$ dargestellt. Das Integral vereinfacht sich, da zum einen das Integral über ungerade Funktionen verschwindet und zum anderen analytisch leichter lösbare Integrale entstehen.

Zwecks Symmetrisierung wird zuerst die Differenz der Fermifunktionen betrachtet. Diese kann man sich anschaulich als eine Linearkombination von einer geraden und einer ungeraden Funktion vorstellen (s. Kasten in Abb. B.1). 
Fall 1:

Spitze und Probe auf gleicher

Temperatur,

Spannungsdifferenz am

Tunnelkontakt

$$
\Delta \mathrm{f}=\mathrm{f}(\varepsilon-\Delta \mu, \mathrm{T})-\mathrm{f}(\varepsilon+\Delta \mu, \mathrm{T})
$$

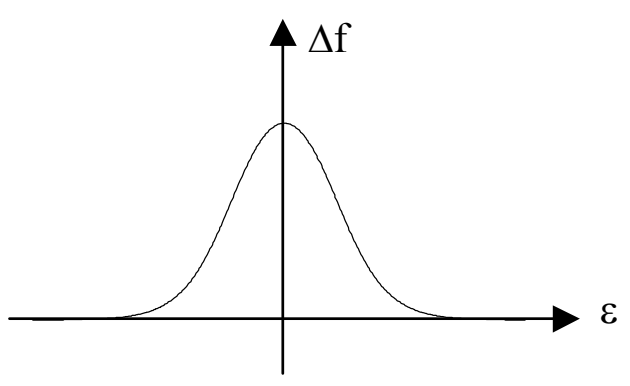

$\rightarrow$ Differenz der Fermifunktionen ist eine gerade Funktion

$\rightarrow$ Tersoff-Hamann-Integral berechnet feldgetriebenen Strom
Fall 2:

Temperaturunterschied zwischen Spitze und Probe, keine Spannungsdifferenz am Tunnelkontakt

$$
\Delta \mathrm{f}=\mathrm{f}\left(\varepsilon, \mathrm{T}_{\mathrm{S}}\right)-\mathrm{f}\left(\varepsilon, \mathrm{T}_{\mathrm{P}}\right)
$$

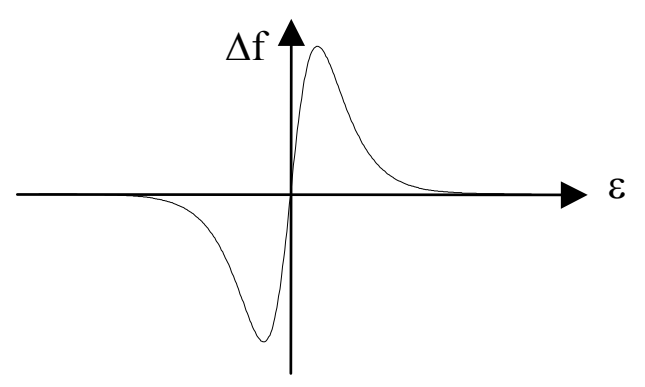

$\rightarrow$ Differenz der Fermifunktionen ist eine ungerade Funktion

$\rightarrow$ Tersoff-Hamann-Integral berechnet thermischen Diffusionsstrom

Abb. B.1 Veranschaulichung der Differenz der Fermifunktionen für zwei Spezialfälle.

Für kleine Potenzialdifferenzen $\mathrm{eU}_{\mathrm{Th}} \ll \mathrm{k}_{\mathrm{B}} \mathrm{T}$ kann die Differenz der Fermifunktionen geschrieben werden zu

$$
\begin{aligned}
& \mathrm{f}\left(\varepsilon+\Delta \mu, \mathrm{T}_{\mathrm{S}}\right)-\mathrm{f}\left(\varepsilon-\Delta \mu, \mathrm{T}_{\mathrm{P}}\right) \\
& =\left[\mathrm{f}\left(\varepsilon, \mathrm{T}_{\mathrm{S}}\right)+\frac{\mathrm{df}\left(\varepsilon, \mathrm{T}_{\mathrm{S}}\right)}{\mathrm{d} \varepsilon} \cdot \Delta \mu\right]-\left[\mathrm{f}\left(\varepsilon, \mathrm{T}_{\mathrm{P}}\right)+\frac{\mathrm{df}\left(\varepsilon, \mathrm{T}_{\mathrm{P}}\right)}{\mathrm{d} \varepsilon} \cdot(-\Delta \mu)\right] \\
& =: \Delta \mathrm{f}_{\mathrm{T}}(\varepsilon)+\overline{\mathrm{f}^{\prime}(\varepsilon)} \cdot 2 \Delta \mu_{\mathrm{ec}}=\Delta \mathrm{f}_{\mathrm{T}}(\varepsilon)+\overline{\mathrm{f}^{\prime}(\varepsilon)} \cdot \mathrm{eU}_{\mathrm{Th}}(\mathrm{x})
\end{aligned}
$$

mit den Vereinbarungen

$$
\overline{f^{\prime}(\varepsilon)}=\frac{1}{2} \cdot\left(\frac{d f\left(\varepsilon, T_{S}\right)}{d \varepsilon}+\frac{d f\left(\varepsilon, T_{P}\right)}{d \varepsilon}\right),
$$

Gleichung (B.4) stellt hier den Fall 1 in Abb. B.1 und Gleichung (B.5) den Fall 2 in Abb. B.1 dar.

Von der Zustandsdichte der Spitze wird angenommen, dass sie sich innerhalb der thermischen Verbreiterung der Fermifunktionen nur schwach ändert und um $\varepsilon=0$ Taylor-entwickelt werden kann: 


$$
\rho_{\mathrm{S}}(\varepsilon)=\left.\rho_{\mathrm{S}}(\varepsilon)\right|_{\varepsilon=0}+\left.\frac{\partial \rho_{\mathrm{S}}(\varepsilon)}{\partial \varepsilon}\right|_{\varepsilon=0} \cdot \varepsilon=\rho_{\mathrm{S} 0}+\rho_{\mathrm{S} 0}^{\prime} \cdot \varepsilon
$$

Die LDOS $\rho_{\mathrm{P}}(\overrightarrow{\mathrm{r}}, \varepsilon)$ der Probe setzt sich zusammen aus einem Anteil von Volumenzuständen und von Oberflächenzuständen. Die LDOS $\rho_{\mathrm{V}}$ der Volumenzustände kann in guter Näherung als konstant in $\mathrm{x}$ - und $\mathrm{y}$-Richtungen parallel zur Oberfläche betrachtet werden, also als eine Funktion $\rho_{\mathrm{V}}(\mathrm{z}, \varepsilon)$. Wie die Zustandsdichte der Spitze kann diese Taylor-entwickelt werden um $\varepsilon=0$ :

$$
\rho_{\mathrm{V}}(\mathrm{z}, \varepsilon)=\left.\rho_{\mathrm{V}}(\mathrm{z}, \varepsilon)\right|_{\varepsilon=0}+\left.\frac{\partial \rho_{\mathrm{V}}(\mathrm{z}, \varepsilon)}{\partial \varepsilon}\right|_{\varepsilon=0} \cdot \varepsilon=\rho_{\mathrm{V} 0}+\rho_{\mathrm{V} 0}^{\prime} \cdot \varepsilon .
$$

Die LDOS $\rho_{\mathrm{OF}}$ der Oberflächenzustände ist in Gleichung (7.5) angegeben zu

$$
\rho_{\mathrm{OF}}(\overrightarrow{\mathrm{r}}, \varepsilon)=\rho_{\mathrm{OF}}(\mathrm{z}) \cdot\left(1-\mathrm{R} \cdot \mathrm{J}_{0}\left(2 \mathrm{k}_{\|}(\varepsilon) \mathrm{x}\right)\right) .
$$

Man beachte, dass (abgesehen vom Interferenzterm) die Oberflächenzustände keine Energieabhängigkeit besitzen, da die Zustandsdichte eines zweidimensionalen Elektronengases konstant bezüglich der Energie ist.

Nach Zusammenfassen von (B.7) und (B.8) ergibt sich für die LDOS $\rho_{P}$ der Probe:

$$
\rho_{\mathrm{P}}(\overrightarrow{\mathrm{r}}, \varepsilon)=\rho_{\mathrm{V}}(\mathrm{z}, \varepsilon)+\rho_{\mathrm{OF}}(\overrightarrow{\mathrm{r}}, \varepsilon)=\rho_{\mathrm{V} 0}+\rho_{\mathrm{V} 0}^{\prime} \cdot \varepsilon+\rho_{\mathrm{OF}} \cdot\left(1-\mathrm{R} \cdot \mathrm{J}_{0}\left(2 \mathrm{k}_{\|}(\varepsilon) \mathrm{x}\right)\right) .
$$

Zur Vereinfachung sei der Gewichtungsfaktor $\mathrm{P}$ eingeführt. $\mathrm{P}$ soll den Anteil der Oberflächenzustände an der gesamten LDOS $\rho_{\mathrm{P} 0}$ bei $\varepsilon=0$ für den Fall einer ungestörten Oberfläche (d.h. einer Oberfläche ohne Interferenzerscheinungen) angeben. P wurde für $\mathrm{Au}(111)$ experimentell bestimmt zu $\mathrm{P} \approx 0.5$ [Cro 1, Bür 1]. Es gilt also

$$
\rho_{\mathrm{OF}}=\mathrm{P} \cdot \rho_{\mathrm{P} 0} \quad \text { und } \quad \rho_{\mathrm{V} 0}=[1-\mathrm{P}] \cdot \rho_{\mathrm{P} 0} .
$$

Einsetzen von (B.10) in (B.9) ergibt mit der Abkürzung C $=\mathrm{P} \cdot \mathrm{R}$ :

$$
\rho_{\mathrm{P}}(\overrightarrow{\mathrm{r}}, \varepsilon)=\rho_{\mathrm{V} 0}^{\prime} \cdot \varepsilon+\rho_{\mathrm{P} 0} \cdot\left(1-\mathrm{C} \cdot \mathrm{J}_{0}\left(2 \mathrm{k}_{\|}(\varepsilon) \mathrm{x}\right)\right) .
$$

Die Amplitudenreflektivität $\mathrm{R}$ der Stufe wurde theoretisch zu etwa $\mathrm{R} \approx 0.4$ ermittelt Hör 1$]$. Diese beiden Konstanten ergeben multipliziert zu C eine Gewichtung für die Amplitude der stehenden Wellen relativ zu einer in $\mathrm{x}$ - und y-Richtung homogenen Hintergrund-LDOS $\rho_{0}$.

Die Ausdrücke (B.3), (B.6) und (B.11) können jetzt in das Integral von (B.2) eingesetzt und ausmultipliziert werden. Die Integrale von Funktionen, die als Produkt einer ungeraden mit einer geraden Funktion auftreten, verschwinden aufgrund ihrer Symmetrie. Weiterhin werden Terme zweiter und höherer Ordnung in E vernachlässigt. Die Auswertung ergibt dann: 
(B.12)

$$
\begin{aligned}
& \mathrm{I}_{\mathrm{T}}=0=\left(\rho_{\mathrm{S} 0} \rho_{\mathrm{V} 0}^{\prime}+\rho_{\mathrm{P} 0} \rho_{\mathrm{S} 0}^{\prime}\right) \cdot \int_{-\infty}^{\infty} \varepsilon \cdot \Delta \mathrm{f}_{\mathrm{T}}(\varepsilon) \cdot \mathrm{d} \varepsilon+\rho_{\mathrm{S} 0} \rho_{\mathrm{P} 0} \cdot \mathrm{eU}_{\mathrm{Th}}(\mathrm{x}) \cdot \int_{-\infty}^{\infty} \overline{\mathrm{f}^{\prime}(\varepsilon)} \cdot \mathrm{d} \varepsilon \\
& -\int_{-\infty}^{\infty}\left(\rho_{\mathrm{S} 0}+\rho_{\mathrm{S} 0}^{\prime} \cdot \varepsilon\right) \cdot \rho_{\mathrm{P} 0} \mathrm{CJ}_{0}\left(2 \mathrm{k}_{\|}(\varepsilon) \mathrm{x}\right) \cdot \Delta \mathrm{f}_{\mathrm{T}}(\varepsilon) \cdot \mathrm{d} \varepsilon \\
& -\int_{-\infty}^{\infty}\left(\rho_{\mathrm{S} 0}+\rho_{\mathrm{S} 0}^{\prime} \cdot \varepsilon\right) \cdot \rho_{\mathrm{P} 0} \mathrm{CJ}_{0}\left(2 \mathrm{k}_{\|}(\varepsilon) \mathrm{x}\right) \cdot \overline{\mathrm{f}^{\prime}(\varepsilon)} \cdot \mathrm{eU}_{\mathrm{Th}}(\mathrm{x}) \cdot \mathrm{d} \varepsilon
\end{aligned}
$$

Der Integrand in $\int_{-\infty}^{\infty} \varepsilon \cdot \Delta \mathrm{f}_{\mathrm{T}}(\varepsilon) \cdot \mathrm{d} \varepsilon$ ist eine gerade Funktion, so dass es ausreicht nur eine Integralhälfte für $\varepsilon>0$ auszurechnen, d.h. es gilt $\int_{-\infty}^{\infty} \varepsilon \cdot \Delta \mathrm{f}_{\mathrm{T}}(\varepsilon) \cdot \mathrm{d} \varepsilon=2 \cdot \int_{0}^{\infty} \varepsilon \cdot \Delta \mathrm{f}_{\mathrm{T}}(\varepsilon) \cdot \mathrm{d} \varepsilon$.

Nach Einsetzen von (B.5) kann dieses Integral nach [Bro, Kap. 1.1.3.4. Nr. 6] mit Hilfe der Substitution $\mathrm{t}=\varepsilon /\left(\mathrm{k}_{\mathrm{B}} \mathrm{T}\right)$ für jeweils $\mathrm{T}=\mathrm{T}_{\mathrm{S}}$ bzw. $\mathrm{T}=\mathrm{T}_{\mathrm{P}}$ gelöst werden:

$$
\begin{aligned}
& \int_{-\infty}^{\infty} \varepsilon \cdot \Delta \mathrm{f}_{\mathrm{T}}(\varepsilon) \cdot \mathrm{d} \varepsilon=2 \mathrm{k}_{\mathrm{B}}^{2} \mathrm{~T}_{\mathrm{S}}^{2} \int_{0}^{\infty} \frac{\mathrm{t} \cdot \mathrm{dt}}{1+\exp (\mathrm{t})}-2 \mathrm{k}_{\mathrm{B}}^{2} \mathrm{~T}_{\mathrm{P}}^{2} \int_{0}^{\infty} \frac{\mathrm{t} \cdot \mathrm{dt}}{1+\exp (\mathrm{t})}=\frac{\pi^{2}}{6} \mathrm{k}_{\mathrm{B}}^{2} \cdot\left(\mathrm{T}_{\mathrm{S}}^{2}-\mathrm{T}_{\mathrm{P}}^{2}\right) \\
& =\frac{\pi^{2}}{3} \mathrm{k}_{\mathrm{B}}^{2} \cdot \Delta \mathrm{T} \cdot \mathrm{T}_{\mathrm{m}},
\end{aligned}
$$

wobei $\Delta \mathrm{T}=\mathrm{T}_{\mathrm{S}}-\mathrm{T}_{\mathrm{P}}$ die Temperaturdifferenz zwischen Spitze und Probe und $\mathrm{T}_{\mathrm{m}}=\left(\mathrm{T}_{\mathrm{S}}+\mathrm{T}_{\mathrm{P}}\right) / 2$ die gemittelte Temperatur angebe. Das Integral im zweiten Term von (B.12) beträgt

$$
\int_{-\infty}^{\infty} \overline{f^{\prime}(\varepsilon)} \cdot d \varepsilon=\int_{-\infty}^{\infty} \frac{1}{2}\left(\frac{\partial \mathrm{f}\left(\varepsilon, \mathrm{T}_{\mathrm{S}}\right)}{\partial \varepsilon}+\frac{\partial \mathrm{f}\left(\varepsilon, \mathrm{T}_{\mathrm{P}}\right)}{\partial \varepsilon}\right) \cdot \mathrm{d} \varepsilon=-1
$$

Wenn sich die Zustandsdichte $\rho_{\mathrm{S}}$ der Spitze nur schwach innerhalb der thermischen Verbreiterung der Fermifunktion ändert, d.h. wenn gilt $4 \mathrm{k}_{\mathrm{B}} \mathrm{T} \cdot \rho_{\mathrm{S} 0}^{\prime} " \rho_{\mathrm{S} 0}$, dann kann in den letzten beiden Integralen von (B.12) der Taylor-Term erster Ordnung, $\rho_{\mathrm{S} 0}^{\prime} \cdot \varepsilon$, vernachlässigt werden. Gleichung (B.12) kann in diesem Fall nach Einsetzen von (B.13) und (B.14) vereinfacht werden zu

$$
\begin{aligned}
\mathrm{I}_{\mathrm{T}}=0= & \left(\rho_{\mathrm{S} 0} \rho_{\mathrm{V} 0}^{\prime}+\rho_{\mathrm{P} 0} \rho_{\mathrm{S} 0}^{\prime}\right) \cdot \frac{\pi^{2}}{3} \mathrm{k}_{\mathrm{B}}^{2} \cdot \Delta \mathrm{T} \cdot \mathrm{T}_{\mathrm{m}} \quad-\rho_{\mathrm{S} 0} \rho_{\mathrm{P} 0} \cdot \mathrm{eU} \mathrm{Th}(\mathrm{x}) \\
& -\rho_{\mathrm{S} 0} \rho_{\mathrm{P} 0} \mathrm{C} \cdot \int_{-\infty}^{\infty} \mathrm{J}_{0}\left(2 \mathrm{k}_{\|}(\varepsilon) \mathrm{x}\right) \cdot \Delta \mathrm{f}_{\mathrm{T}}(\varepsilon) \cdot \mathrm{d} \varepsilon \\
& -\rho_{\mathrm{S} 0} \rho_{\mathrm{P} 0} \mathrm{C} \cdot \mathrm{eU} \mathrm{U}_{\mathrm{Th}}(\mathrm{x}) \cdot \int_{-\infty}^{\infty} \mathrm{J}_{0}\left(2 \mathrm{k}_{\|}(\varepsilon) \mathrm{x}\right) \cdot \overline{\mathrm{f}^{\prime}(\varepsilon)} \cdot \mathrm{d} \varepsilon
\end{aligned}
$$


Aufgelöst nach $\mathrm{U}_{\mathrm{Th}}(\mathrm{x})$ ergibt sich

$$
\mathrm{U}_{\mathrm{Th}}(\mathrm{x})=\frac{\left(\rho_{\mathrm{S} 0} \rho_{\mathrm{V} 0}^{\prime}+\rho_{\mathrm{P} 0} \rho_{\mathrm{S} 0}^{\prime}\right) \cdot \frac{\pi^{2}}{3} \mathrm{k}_{\mathrm{B}}^{2} \cdot \Delta \mathrm{T} \cdot \mathrm{T}_{\mathrm{m}}-\rho_{\mathrm{S} 0} \rho_{\mathrm{P} 0} \mathrm{C} \cdot \int_{-\infty}^{\infty} \mathrm{J}_{0}\left(2 \mathrm{k}_{\|}(\varepsilon) \mathrm{x}\right) \cdot \Delta \mathrm{f}_{\mathrm{T}}(\varepsilon) \cdot \mathrm{d} \varepsilon}{\rho_{\mathrm{S} 0} \rho_{\mathrm{P} 0} \cdot \mathrm{e} \cdot\left(1+\mathrm{C} \cdot \int_{-\infty}^{\infty} \mathrm{J}_{0}\left(2 \mathrm{k}_{\|}(\varepsilon) \mathrm{x}\right) \cdot \overline{\left.\mathrm{f}^{\prime}(\varepsilon) \cdot \mathrm{d} \varepsilon\right)}\right.} .
$$

Mit $\rho_{\mathrm{V} 0}^{\prime} / \rho_{\mathrm{P} 0}=(1-\mathrm{P}) \rho_{\mathrm{V} 0}^{\prime} / \rho_{\mathrm{v} 0}$ nach (B.10) $\operatorname{kann}(\mathrm{B} .16)$ vereinfacht werden zu

$$
\mathrm{U}_{\mathrm{Th}}(\mathrm{x})=\frac{\left((1-\mathrm{P}) \frac{\rho_{\mathrm{V} 0}^{\prime}}{\rho_{\mathrm{V} 0}}+\frac{\rho_{\mathrm{S} 0}^{\prime}}{\rho_{\mathrm{S} 0}}\right) \cdot \frac{\pi^{2}}{3 \mathrm{e}} \mathrm{k}_{\mathrm{B}}^{2} \cdot \Delta \mathrm{T} \cdot \mathrm{T}_{\mathrm{m}}-\frac{\mathrm{C}}{\mathrm{e}} \cdot \int_{-\infty}^{\infty} \mathrm{J}_{0}\left(2 \mathrm{k}_{\|}(\varepsilon) \mathrm{x}\right) \cdot \Delta \mathrm{f}_{\mathrm{T}}(\varepsilon) \cdot \mathrm{d} \varepsilon}{1+\mathrm{C} \cdot \int_{-\infty}^{\infty} \mathrm{J}_{0}\left(2 \mathrm{k}_{\|}(\varepsilon) \mathrm{x}\right) \cdot \overline{\mathrm{f}^{\prime}(\varepsilon) \cdot \mathrm{d} \varepsilon}} .
$$

Die Integrale in Gleichung (B.17) können analytisch näherungsweise gelöst werden, indem nach Anhang $\mathrm{C}$ die Besselfunktion $\mathrm{J}_{0}$ durch eine Linearkombination aus einer Sinus- und Kosinusfunktion ersetzt wird:

$$
\mathrm{J}_{0}\left(2 \mathrm{k}_{\|}(\varepsilon) \mathrm{x}\right) \approx \mathrm{J}_{0}\left(2 \mathrm{k}_{\mathrm{F}} \mathrm{x}\right) \cdot \cos (\mathrm{b} \varepsilon)-\mathrm{J}_{1}\left(2 \mathrm{k}_{\mathrm{F}} \mathrm{x}\right) \cdot \sin (\mathrm{b} \varepsilon)
$$

mit $\mathrm{J}_{1}$ als Besselfunktion erster Gattung erster Ordnung und der Abkürzung $\mathrm{b}=\mathrm{k}_{\mathrm{F}} \mathrm{x} / \varepsilon_{\mathrm{F}}$. In dieser Näherung wird unter der Annahme $\varepsilon_{\mathrm{F}} \gg \mathrm{k}_{\mathrm{B}} \mathrm{T}$ die Wellenzahl $\mathrm{k}(\varepsilon)$ um $\varepsilon=0$ linearisiert und das $1 / \sqrt{\varepsilon}$-Abklingen der Besselfunktion vernachlässigt (s. Anhang $C$ ). Unter der Berücksichtigung, dass $\Delta \mathrm{f}_{\mathrm{T}}(\varepsilon)$ nach (B.5) eine ungerade Funktion ist, kann das Integral im Zähler von (B.17) genähert werden zu

$$
\begin{aligned}
& \int_{-\infty}^{\infty} \mathrm{J}_{0}\left(2 \mathrm{k}_{\|}(\varepsilon) \mathrm{x}\right) \cdot \Delta \mathrm{f}_{\mathrm{T}}(\varepsilon) \cdot \mathrm{d} \varepsilon \\
& \approx \mathrm{J}_{0}\left(2 \mathrm{k}_{\mathrm{F}} \mathrm{x}\right) \cdot \int_{-\infty}^{\infty} \cos (\mathrm{b} \varepsilon) \cdot \Delta \mathrm{f}_{\mathrm{T}}(\varepsilon) \cdot \mathrm{d} \varepsilon-\mathrm{J}_{1}\left(2 \mathrm{k}_{\mathrm{F}} \mathrm{x}\right) \cdot \int_{-\infty}^{\infty} \sin (\mathrm{b} \varepsilon) \cdot \Delta \mathrm{f}_{\mathrm{T}}(\varepsilon) \cdot \mathrm{d} \varepsilon \\
& =-2 \cdot \mathrm{J}_{1}\left(2 \mathrm{k}_{\mathrm{F}} \mathrm{x}\right) \cdot \int_{0}^{\infty} \sin (\mathrm{b} \varepsilon) \cdot\left[\mathrm{f}\left(\varepsilon, \mathrm{T}_{\mathrm{S}}\right)-\mathrm{f}\left(\varepsilon, \mathrm{T}_{\mathrm{P}}\right)\right] \cdot \mathrm{d} \varepsilon
\end{aligned}
$$

Letzteres Integral kann nach [Gra, Gl. 3.911, Nr.1] gelöst werden zu

$$
\int_{0}^{\infty} \sin (\mathrm{b} \varepsilon) \cdot \mathrm{f}(\varepsilon) \cdot \mathrm{d} \varepsilon=\frac{1}{2 \mathrm{~b}}-\frac{\mathrm{s}}{2 \mathrm{~b} \cdot \sinh (\mathrm{s})}
$$

mit der Abkürzung $\mathrm{s}=\pi \cdot \mathrm{k}_{\mathrm{B}} \mathrm{T} \cdot \mathrm{k}_{\mathrm{F}} \mathrm{x} / \varepsilon_{\mathrm{F}}$. Das Integral im Zähler von (B.17) wird also gelöst zu

$$
\frac{\mathrm{C}}{\mathrm{e}} \cdot \int_{-\infty}^{\infty} \mathrm{J}_{0}\left(2 \mathrm{k}_{\|}(\varepsilon) \mathrm{x}\right) \cdot \Delta \mathrm{f}_{\mathrm{T}}(\varepsilon) \cdot \mathrm{d} \varepsilon \approx-\mathrm{C} \cdot \mathrm{J}_{1}\left(2 \mathrm{k}_{\mathrm{F}} \mathrm{x}\right) \cdot \mathrm{h}_{\mathrm{z}}(\mathrm{x})
$$


mit den Abkürzungen

$$
\begin{aligned}
& \mathrm{h}_{\mathrm{z}}(\mathrm{x})=\frac{\varepsilon_{\mathrm{F}}}{\mathrm{e} \cdot \mathrm{k}_{\mathrm{F}} \mathrm{x}} \cdot\left(\frac{\mathrm{s}_{\mathrm{P}}}{\sinh \left(\mathrm{s}_{\mathrm{P}}\right)}-\frac{\mathrm{s}_{\mathrm{S}}}{\sinh \left(\mathrm{s}_{\mathrm{S}}\right)}\right), \\
& \mathrm{s}_{\mathrm{P}}=\pi \cdot \mathrm{k}_{\mathrm{B}} \mathrm{T}_{\mathrm{P}} \cdot \mathrm{k}_{\mathrm{F}} \mathrm{x} / \varepsilon_{\mathrm{F}},
\end{aligned}
$$$$
\mathrm{s}_{\mathrm{S}}=\pi \cdot \mathrm{k}_{\mathrm{B}} \mathrm{T}_{\mathrm{S}} \cdot \mathrm{k}_{\mathrm{F}} \mathrm{x} / \varepsilon_{\mathrm{F}} .
$$

Auf ähnliche Weise kann das Integral im Nenner von (B.17) gelöst werden. Unter der Berücksichtigung, dass $\overline{\mathrm{f}^{\prime}(\varepsilon)}$ nach (B.4) eine gerade Funktion ist, gilt

$$
\begin{aligned}
& \int_{-\infty}^{\infty} \mathrm{J}_{0}\left(2 \mathrm{k}_{\|}(\varepsilon) \mathrm{x}\right) \cdot \overline{\mathrm{f}^{\prime}(\varepsilon)} \cdot \mathrm{d} \varepsilon \\
& \approx \mathrm{J}_{0}\left(2 \mathrm{k}_{\mathrm{F}} \mathrm{x}\right) \cdot \int_{-\infty}^{\infty} \cos (\mathrm{b} \varepsilon) \cdot \overline{\mathrm{f}^{\prime}(\varepsilon)} \cdot \mathrm{d} \varepsilon-\mathrm{J}_{1}\left(2 \mathrm{k}_{\mathrm{F}} \mathrm{x}\right) \cdot \int_{-\infty}^{\infty} \sin (\mathrm{b} \varepsilon) \cdot \overline{\mathrm{f}^{\prime}(\varepsilon)} \cdot \mathrm{d} \varepsilon \\
& =2 \cdot \mathrm{J}_{0}\left(2 \mathrm{k}_{\mathrm{F}} \mathrm{x}\right) \cdot \int_{0}^{\infty} \cos (\mathrm{b} \varepsilon) \cdot \frac{1}{2} \cdot\left(\frac{\mathrm{df}\left(\varepsilon, \mathrm{T}_{\mathrm{S}}\right)}{\mathrm{d} \varepsilon}+\frac{\mathrm{df}\left(\varepsilon, \mathrm{T}_{\mathrm{P}}\right)}{\mathrm{d} \varepsilon}\right) \cdot \mathrm{d} \varepsilon .
\end{aligned}
$$

Durch partielle Integration und mit Hilfe von (B.20) erhält man

$$
\begin{aligned}
& \int_{0}^{\infty} \cos (\mathrm{b} \varepsilon) \cdot \frac{\mathrm{df}(\varepsilon, \mathrm{T})}{\mathrm{d} \varepsilon} \cdot \mathrm{d} \varepsilon=[\cos (\mathrm{b} \varepsilon) \cdot \mathrm{f}(\varepsilon, \mathrm{T})]_{0}^{\infty}+\mathrm{b} \cdot \int_{0}^{\infty} \sin (\mathrm{b} \varepsilon) \cdot \mathrm{f}(\varepsilon, \mathrm{T}) \cdot \mathrm{d} \varepsilon \\
& =-\frac{\mathrm{s}}{2 \cdot \sinh (\mathrm{s})}
\end{aligned}
$$

Einsetzen von (B.26) in (B.25) ergibt

$$
\int_{-\infty}^{\infty} \mathrm{J}_{0}\left(2 \mathrm{k}_{\|}(\varepsilon) \mathrm{x}\right) \cdot \overline{\mathrm{f}^{\prime}(\varepsilon)} \cdot \mathrm{d} \varepsilon \approx-\mathrm{J}_{0}\left(2 \mathrm{k}_{\mathrm{F}} \mathrm{x}\right) \cdot \mathrm{h}_{\mathrm{N}}(\mathrm{x})
$$

mit der Abkürzung

$$
\mathrm{h}_{\mathrm{N}}(\mathrm{x})=\frac{1}{2} \cdot\left(\frac{\mathrm{s}_{\mathrm{S}}}{\sinh \left(\mathrm{s}_{\mathrm{S}}\right)}+\frac{\mathrm{s}_{\mathrm{P}}}{\sinh \left(\mathrm{s}_{\mathrm{P}}\right)}\right),
$$

wobei $\mathrm{s}_{\mathrm{S}}$ und $\mathrm{s}_{\mathrm{P}}$ in (B.23) und (B.24) definiert sind.

Die Gleichungen (B.21) und (B.27) eingesetzt in (B.17) ergeben die analytische Näherung für die Thermospannung:

$$
\mathrm{U}_{\mathrm{Th}}(\mathrm{x})=\frac{\left((1-\mathrm{P}) \frac{\rho_{\mathrm{V} 0}^{\prime}}{\rho_{\mathrm{V} 0}}+\frac{\rho_{\mathrm{S} 0}^{\prime}}{\rho_{\mathrm{S} 0}}\right) \cdot \frac{\pi^{2}}{3 \mathrm{e}} \mathrm{k}_{\mathrm{B}}^{2} \cdot \Delta \mathrm{T} \cdot \mathrm{T}_{\mathrm{m}}+\mathrm{C} \cdot \mathrm{J}_{1}\left(2 \mathrm{k}_{\mathrm{F}} \mathrm{x}\right) \cdot \mathrm{h}_{\mathrm{Z}}(\mathrm{x})}{1-\mathrm{C} \cdot \mathrm{J}_{0}\left(2 \mathrm{k}_{\mathrm{F}} \mathrm{x}\right) \cdot \mathrm{h}_{\mathrm{N}}(\mathrm{x})} .
$$


Wenn die LDOS der Volumenzustände und die Zustandsdichte der Spitze vernachlässigt werden, dann vereinfacht sich (B.29) zu

$$
\mathrm{U}_{\mathrm{Th}}(\mathrm{x})=\frac{\mathrm{C} \cdot \mathrm{J}_{1}\left(2 \mathrm{k}_{\mathrm{F}} \mathrm{x}\right) \cdot \mathrm{h}_{\mathrm{Z}}(\mathrm{x})}{1-\mathrm{C} \cdot \mathrm{J}_{0}\left(2 \mathrm{k}_{\mathrm{F}} \mathrm{x}\right) \cdot \mathrm{h}_{\mathrm{N}}(\mathrm{x})} .
$$

Die Extrema der Besselfunktion $\mathrm{J}_{1}$ fallen nahezu mit den Nullstellen von $\mathrm{J}_{0}$ zusammen, so dass der Nennerterm die Einhüllendenfunktion von $\mathrm{U}_{\mathrm{Th}}(\mathrm{x})$ nur unwesentlich beeinflusst.

Wenn die Einhüllende der Besselfunktion $\mathrm{J}_{1}$ in guter Näherung $\mathrm{zu} 1 / \sqrt{\pi \mathrm{k}_{\mathrm{F}} \mathrm{x}}$ angenommen wird [Bro, Kap. 3.3.1.3.4], dann besitzt die Einhüllende $\mathrm{U}_{\mathrm{env}}(\mathrm{x})$ von $\mathrm{U}_{\mathrm{Th}}(\mathrm{x})$ die einfache Form

$$
\mathrm{U}_{\mathrm{Env}}(\mathrm{x}) \approx \frac{\mathrm{C}}{\sqrt{\pi \mathrm{k}_{\mathrm{F}} \mathrm{x}}} \cdot \mathrm{h}_{\mathrm{z}}(\mathrm{x})
$$

\section{Die Thermospannung für kleine Abstände von der Stufe}

Nahe der Stufenkante lässt sich Gleichung (B.30) im Grenzfall $\mathrm{x} \rightarrow 0$ nähern, indem die Funktionen $\mathrm{h}_{\mathrm{Z}}(\mathrm{x})$ und $\mathrm{h}_{\mathrm{N}}(\mathrm{x})$ Gleichung (B.22) und (B.28) in eine Potenzreihe (Taylorentwicklung bei $\varepsilon=0$ ) entwickelt werden:

$$
\begin{aligned}
& \mathrm{h}_{\mathrm{Z}}(\mathrm{x}) \approx \frac{\varepsilon_{\mathrm{F}}}{\mathrm{e} \cdot \mathrm{k}_{\mathrm{F}} \mathrm{x}} \cdot \frac{\left(\mathrm{s}_{\mathrm{S}}^{2}-\mathrm{s}_{\mathrm{P}}^{2}\right)}{6}=\pi^{2} \mathrm{k}_{\mathrm{B}}^{2} \cdot \Delta \mathrm{T} \cdot \mathrm{T}_{\mathrm{m}} \cdot \frac{\mathrm{k}_{\mathrm{F}} \mathrm{x}}{3 \mathrm{e} \cdot \varepsilon_{\mathrm{F}}}, \\
& \mathrm{h}_{\mathrm{N}}(\mathrm{x}) \approx 1 .
\end{aligned}
$$

Damit wird Gleichung (B.30) bzw. (B.30a) genähert zu

$$
\mathrm{U}_{\mathrm{Th}}(\mathrm{x}) \approx \frac{\pi^{2} \cdot \mathrm{k}_{\mathrm{B}}^{2} \cdot \Delta \mathrm{T} \cdot \mathrm{T}_{\mathrm{m}} \cdot \mathrm{C} \cdot \mathrm{k}_{\mathrm{F}} \mathrm{x} \cdot \mathrm{J}_{1}\left(2 \mathrm{k}_{\mathrm{F}} \mathrm{x}\right)}{3 \mathrm{e} \cdot \varepsilon_{\mathrm{F}} \cdot\left[1-\mathrm{C} \cdot \mathrm{J}_{0}\left(2 \mathrm{k}_{\mathrm{F}} \mathrm{x}\right)\right]}
$$

mit einer Einhüllenden

$$
\mathrm{U}_{\mathrm{Env}}(\mathrm{x}) \approx \frac{\pi^{3 / 2} \cdot \mathrm{k}_{\mathrm{B}}^{2} \cdot \Delta \mathrm{T} \cdot \mathrm{T}_{\mathrm{m}} \cdot \mathrm{C}}{3 \mathrm{e} \cdot \varepsilon_{\mathrm{F}}} \cdot \sqrt{\mathrm{k}_{\mathrm{F}} \mathrm{x}} .
$$

\section{Näherung für große Abstände von der Stufe}

Für große Abstände von der Stufe, $\mathrm{x} \rightarrow \infty$, geht der Nenner von (B.30) gegen eins. Die Einhüllende $\mathrm{h}_{\mathrm{Z}}(\mathrm{x})$ Gl. (B.22) im Zähler von (B.30) kann mit $\sinh (\mathrm{s}) \rightarrow \exp (\mathrm{s}) / 2$ genähert werden zu

$$
\mathrm{h}_{\mathrm{Z}}(\mathrm{x}) \rightarrow \frac{2 \varepsilon_{\mathrm{F}}}{\mathrm{e} \cdot \mathrm{k}_{\mathrm{F}} \mathrm{x}} \cdot\left(\frac{\mathrm{s}_{\mathrm{P}}}{\exp \left(\mathrm{s}_{\mathrm{P}}\right)}-\frac{\mathrm{s}_{\mathrm{S}}}{\exp \left(\mathrm{s}_{\mathrm{S}}\right)}\right)
$$

für $\mathrm{s}_{\mathrm{S}}, \mathrm{s}_{\mathrm{P}} \gg 1$. 
Es sei $\mathrm{s}_{\min }=\min \left(\mathrm{s}_{\mathrm{S}}, \mathrm{s}_{\mathrm{P}}\right)$ und $\mathrm{s}_{\max }=\max \left(\mathrm{s}_{\mathrm{S}}, \mathrm{s}_{\mathrm{P}}\right)$, dann kann (B.34) umgeschrieben werden zu

$$
\left|\mathrm{h}_{\mathrm{Z}}(\mathrm{x})\right| \rightarrow \frac{2 \varepsilon_{\mathrm{F}}}{\mathrm{e} \cdot \mathrm{k}_{\mathrm{F}} \mathrm{x}} \cdot \frac{\mathrm{s}_{\min }}{\exp \left(\mathrm{s}_{\min }\right)}\left[1-\frac{\mathrm{s}_{\max }}{\mathrm{s}_{\min }} \cdot \exp \left(\mathrm{s}_{\min }-\mathrm{s}_{\max }\right)\right]
$$

Für $\mathrm{s}_{\max }-\mathrm{s}_{\min } \gg 1$ kann (B.35) weiter genähert werden zu

$$
\left|\mathrm{h}_{\mathrm{Z}}(\mathrm{x})\right| \rightarrow \frac{2 \varepsilon_{\mathrm{F}}}{\mathrm{e} \cdot \mathrm{k}_{\mathrm{F}} \mathrm{x}} \cdot \frac{\mathrm{s}_{\min }}{\exp \left(\mathrm{s}_{\min }\right)}
$$

Mit $\mathrm{s}_{\min }=\pi \cdot \mathrm{k}_{\mathrm{F}} \mathrm{x} \cdot \mathrm{k}_{\mathrm{B}} \mathrm{T}_{\min } / \varepsilon_{\mathrm{F}}$ ist der Gültigkeitsbereich von (B.36)

$$
\begin{aligned}
& \mathrm{S}_{\text {max }}-\mathrm{s}_{\text {min }}=\pi \cdot \mathrm{k}_{\mathrm{F}} \mathrm{x} \cdot \mathrm{k}_{\mathrm{B}}\left(\mathrm{T}_{\max }-\mathrm{T}_{\text {min }}\right) / \varepsilon_{\mathrm{F}}=\pi \cdot \mathrm{k}_{\mathrm{F}} \mathrm{x} \cdot \mathrm{k}_{\mathrm{B}}|\Delta \mathrm{T}| / \varepsilon_{\mathrm{F}} \gg 1 \\
& \Rightarrow \quad \mathrm{k}_{\mathrm{F}} \mathrm{x} \gg \frac{\varepsilon_{\mathrm{F}}}{\pi \cdot \mathrm{k}_{\mathrm{B}}|\Delta \mathrm{T}|} .
\end{aligned}
$$

Einsetzen von (B.36) in (B.30a) ergibt die Einhüllende für große Abstände von der Stufe:

$$
\mathrm{U}_{\text {Env }} \rightarrow \frac{2 \mathrm{C}}{\mathrm{e}} \cdot \sqrt{\frac{\pi}{\mathrm{k}_{\mathrm{F}} \mathrm{x}}} \cdot \frac{\mathrm{k}_{\mathrm{B}} \mathrm{T}_{\min }}{\exp \left(\pi \cdot \mathrm{k}_{\mathrm{F}} \mathrm{x} \cdot \mathrm{k}_{\mathrm{B}} \mathrm{T}_{\min } / \varepsilon_{\mathrm{F}}\right)} .
$$

\section{Die Thermospannung im Bereich maximaler Oszillationsamplitude}

Es gibt einen charakteristischen Abstand von der Stufe, bei dem die Oszillationsamplitude maximal wird. Bei diesem Abstand $\mathrm{x}_{\mathrm{m}}$ entspricht der energetische Abstand zwischen aufeinanderfolgenden LDOS Minima und Maxima, $\Delta \varepsilon_{\mathrm{x}} / 2$, ungefähr der thermischen Verbreiterung $8 \mathrm{k}_{\mathrm{B}} \mathrm{T}_{\max }$ (s. Abb. 8.11). Es muss der Abstand $\mathrm{x}_{\mathrm{m}}$ gefunden werden, bei dem die LDOS für die Energie $\varepsilon=+4 \mathrm{k}_{\mathrm{B}} \mathrm{T}_{\max }$ genau einen halben Wellenzyklus mehr besitzt als die LDOS bei $\varepsilon=-4 \mathrm{k}_{\mathrm{B}} \mathrm{T}_{\max }$. Es gilt also

$$
\mathrm{x}_{\mathrm{m}}=\mathrm{n} \cdot \lambda_{\varepsilon}\left(\varepsilon_{-}\right)=(\mathrm{n}+1 / 2) \cdot \lambda_{\varepsilon}\left(\varepsilon_{+}\right) .
$$

Hier ist $\mathrm{n}$ die Anzahl der Wellenzyklen und $\lambda_{\varepsilon}$ ist die Wellenlänge der LDOS Oszillationen in x-Richtung bei den Energien $\varepsilon_{+}=+4 \mathrm{k}_{\mathrm{B}} \mathrm{T}_{\max }$ und $\varepsilon_{-}=-4 \mathrm{k}_{\mathrm{B}} \mathrm{T}_{\max }$. Für $\lambda_{\varepsilon}(\varepsilon)$ gilt

$$
\lambda_{\varepsilon}(\varepsilon)=\frac{2 \pi}{2 \mathrm{k}(\varepsilon)}=\frac{\pi \hbar}{\sqrt{2 \mathrm{~m}^{*}\left(\varepsilon+\varepsilon_{\mathrm{F}}\right)}} .
$$

Einsetzen von (B.40) in (B.39) ergibt nach $\mathrm{n}$ aufgelöst

$$
\mathrm{n}=\frac{1}{2} \cdot \frac{\sqrt{\varepsilon_{\mathrm{F}}+\varepsilon_{-}}}{\sqrt{\varepsilon_{\mathrm{F}}+\varepsilon_{+}}-\sqrt{\varepsilon_{\mathrm{F}}+\varepsilon_{-}}} .
$$

Einsetzen von (B.41) in (B.39) ergibt 
(B.42)

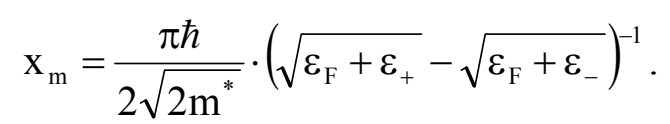

Für kleine Temperaturunterschiede gilt $\varepsilon_{+} \ll \varepsilon_{\mathrm{F}}$, so dass mit der Entwicklung

$$
\sqrt{\varepsilon_{\mathrm{F}}+\varepsilon_{ \pm}} \approx \sqrt{\varepsilon_{\mathrm{F}}} \pm \frac{\varepsilon_{ \pm}}{2 \sqrt{\varepsilon_{\mathrm{F}}}}
$$

eine Näherung für den Abstand $\mathrm{x}_{\mathrm{m}}$ des Oszillations-Maximums gefunden wird:

$$
\mathrm{x}_{\mathrm{m}} \approx \frac{\pi \hbar}{2 \sqrt{2 \mathrm{~m}^{*}}} \cdot\left(\frac{\varepsilon_{+}}{2 \sqrt{\varepsilon_{\mathrm{F}}}}-\frac{\varepsilon_{-}}{2 \sqrt{\varepsilon_{\mathrm{F}}}}\right)^{-1}=\frac{\pi \hbar \cdot \sqrt{\varepsilon_{\mathrm{F}}}}{8 \mathrm{k}_{\mathrm{B}} \mathrm{T}_{\max } \sqrt{2 \mathrm{~m}^{*}}}=\frac{\pi \varepsilon_{\mathrm{F}}}{8 \mathrm{k}_{\mathrm{B}} \mathrm{T}_{\max }} \cdot \frac{1}{\mathrm{k}_{\mathrm{F}}} .
$$




\section{Anhang C: Näherung der Besselfunktion in Fermi-Integralen}

Gesucht ist eine Vereinfachung für die Besselfunktion erster Gattung nullter Ordnung, $\mathrm{J}_{0}\left(2 \cdot \mathrm{k}_{\|}(\varepsilon) \cdot \mathrm{x}\right)$, in einem Fermi-Integral der Form $\int_{-\infty}^{\infty} \mathrm{J}_{0} \cdot \mathrm{f}^{\prime}(\varepsilon) \cdot \mathrm{d} \varepsilon$. Hierbei gibt $\mathrm{k}_{\|}(\varepsilon)$ die Dispersionsrelation nach der Wellenzahl $\mathrm{k}_{\|}$aufgelöst an, welche nach Gl. (6.1) gegeben ist zu

$$
\mathrm{k}_{\|}(\varepsilon)=\sqrt{2 \mathrm{~m}^{*}\left(\varepsilon+\varepsilon_{\mathrm{F}}\right)} / \hbar
$$

$\varepsilon$ sei die Energie relativ zum elektrochemischen Potenzial $\mu_{\mathrm{ec}}$, und $\varepsilon_{\mathrm{F}}$ sei die Energiedifferenz zwischen $\mu_{\mathrm{ec}}$ und der Bandunterkante der Oberflächenzustände. $\mathrm{f}^{\prime}(\varepsilon)$ sei eine Funktion (z.B. die Energieableitung der Fermifunktion), welche in einem Bereich von $\pm 4 \mathrm{k}_{\mathrm{B}} \mathrm{T}$ um $\varepsilon=0$,gepeakt“ sei, d.h. es wird die Annahme gemacht, dass das Integral außerhalb dieses Bereiches vernachlässigbar klein wird.

Gesucht ist eine Näherung für $\mathrm{J}_{0}$ im Bereich der thermischen Verbreiterung der Fermifunktion $\pm 4 \mathrm{k}_{\mathrm{B}} \mathrm{T}$ um das elektrochemische Potenzial bei $\varepsilon=0$ herum (s. Abb. C.1). Dazu werde im folgenden $4 \mathrm{k}_{\mathrm{B}} \mathrm{T} \ll \varepsilon_{\mathrm{F}}$ angenommen.

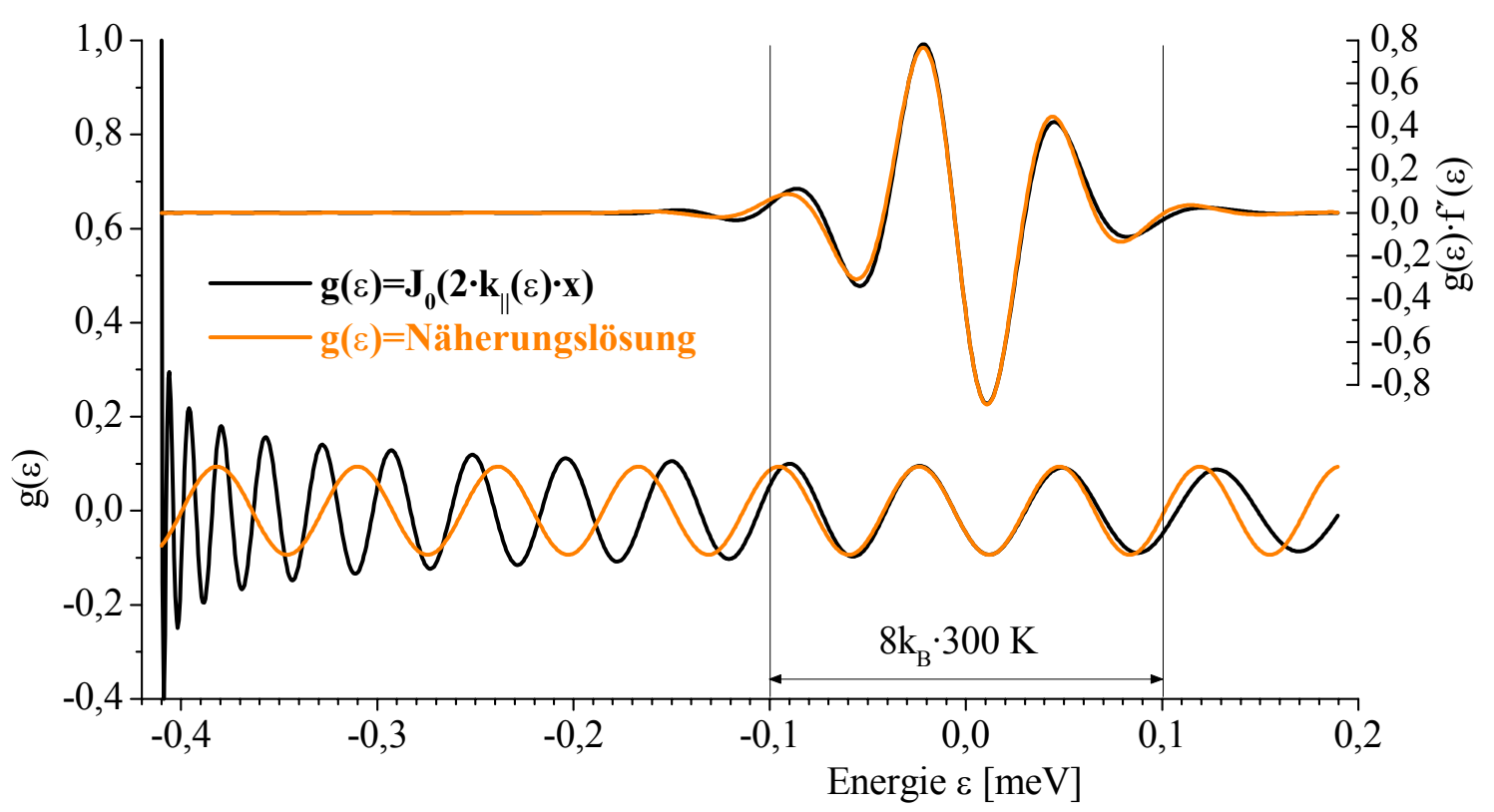

Abb. C.1 Unterer Graph: Vergleich der Besselfunktion $J_{0}(\varepsilon)(-)$ mit der Näherung nach Gleichung C.10) (_) für $\mathrm{x}=20 \mathrm{~nm}$. Oberer Graph: Dieselben Funktionen nach Multiplikation mit $\mathrm{f}^{\prime}(\varepsilon)$ bei einer Temperatur von $\mathrm{T}=300 \mathrm{~K}$

Die Besselfunktion besteht im wesentlichen aus einem oszillatorischen Anteil, welcher im Bereich der thermischen Verbreiterung als Linearkombination aus einer Sinus- und Kosinusfunktion genähert werden kann. Es wird der Ansatz 


$$
\mathrm{J}_{0}\left(2 \cdot \mathrm{k}_{\|}(\varepsilon) \cdot \mathrm{x}\right) \approx \mathrm{A} \cdot \cos (\mathrm{b} \varepsilon)+\mathrm{B} \cdot \sin (\mathrm{b} \varepsilon)
$$

verwendet, wobei A, B und b noch zu bestimmende (nur von x abhängige) Größen sind.

\section{Bestimmung von b:}

Für $4 \mathrm{k}_{\mathrm{B}} \mathrm{T} \ll \varepsilon_{\mathrm{F}}$ kann $\mathrm{k}_{\|}(\varepsilon)$ um $\varepsilon=0$ linearisiert werden, d.h. in eine Taylorreihe erster Ordnung entwickelt werden:

$$
\left.\mathrm{k}_{\|}(\varepsilon) \approx \mathrm{k}_{\|}(\varepsilon)\right|_{\varepsilon=0}+\left.\frac{\mathrm{dk}_{\|}(\varepsilon)}{\mathrm{d} \varepsilon}\right|_{\varepsilon=0} \cdot \varepsilon=\mathrm{k}_{\mathrm{F}}+\frac{\mathrm{k}_{\mathrm{F}}}{2 \varepsilon_{\mathrm{F}}} \cdot \varepsilon
$$

mit $\mathrm{k}_{\mathrm{F}}=\left.\mathrm{k}_{\|}(\varepsilon)\right|_{\varepsilon=0}=\sqrt{2 \mathrm{~m} \varepsilon_{\mathrm{F}}} / \hbar$. Das Argument der Besselfunktion wird dann $\mathrm{zu}$

$$
2 \cdot \mathrm{k}_{\|}(\varepsilon) \cdot \mathrm{x} \approx 2 \mathrm{k}_{\mathrm{F}} \mathrm{x}+\frac{\mathrm{k}_{\mathrm{F}} \mathrm{x}}{\varepsilon_{\mathrm{F}}} \cdot \varepsilon
$$

Der Term $2 \mathrm{k}_{\mathrm{F}} \mathrm{x}$ gibt eine Phase an, während die Periodizität in $\varepsilon$ gegeben ist $\mathrm{zu}$

$$
\mathrm{b}=\frac{\mathrm{k}_{\mathrm{F}} \mathrm{x}}{\varepsilon_{\mathrm{F}}}
$$

\section{Bestimmung von A und B:}

Der Wert von A wird direkt durch Einsetzen von $\varepsilon=0$ in (C.2) $\mathrm{zu}$

$$
\mathrm{A}=\mathrm{J}_{0}\left(2 \mathrm{k}_{\mathrm{F}} \mathrm{x}\right)
$$

bestimmt. Der Wert von B wird gefunden, wenn Gleichung (C.2) nach $\varepsilon$ differenziert wird und $\varepsilon=0$ gesetzt wird. Nach [Bro, Kap. 3.3.1.3.4] gilt $\mathrm{dJ}_{0}(\mathrm{~s}) / \mathrm{ds}=-\mathrm{J}_{1}(\mathrm{~s})$, wobei $\mathrm{J}_{1}$ die Besselfunktion erster Gattung erster Ordnung ist. Für die linke Seite von (C.2) ergibt sich:

$$
\left.\frac{\mathrm{dJ}_{0}\left(2 \mathrm{k}_{\|}(\varepsilon) \mathrm{x}\right)}{\mathrm{d} \varepsilon}\right|_{\varepsilon=0}=-\left.\mathrm{J}_{1}\left(2 \mathrm{k}_{\mathrm{F}} \mathrm{x}\right) \cdot 2 \mathrm{x} \cdot \frac{\mathrm{dk}_{\|}(\varepsilon)}{\mathrm{d} \varepsilon}\right|_{\varepsilon=0}=-\mathrm{J}_{1}\left(2 \mathrm{k}_{\mathrm{F}} \mathrm{x}\right) \cdot \frac{\mathrm{k}_{\mathrm{F}} \mathrm{x}}{\varepsilon_{\mathrm{F}}}
$$

und für die rechte Seite von (C.2)

$$
-\left.\mathrm{A} \cdot \mathrm{b} \cdot \sin (\mathrm{b} \varepsilon)\right|_{\varepsilon=0}+\left.\mathrm{B} \cdot \mathrm{b} \cdot \cos (\mathrm{b} \varepsilon)\right|_{\varepsilon=0}=\mathrm{B} \cdot \frac{\mathrm{k}_{\mathrm{F}} \mathrm{x}}{\varepsilon_{\mathrm{F}}} .
$$

Der Vergleich von (C.7) mit (C.8) ergibt

$$
\mathrm{B}=-\mathrm{J}_{1}\left(2 \mathrm{k}_{\mathrm{F}} \mathrm{x}\right) \text {. }
$$

Eingesetzt in (C.2) ergibt sich also folgende Näherung für die Besselfunktion um $\varepsilon=0$ :

$$
\mathrm{J}_{0}\left(2 \mathrm{k}_{\|}(\varepsilon) \mathrm{x}\right) \approx \mathrm{J}_{0}\left(2 \mathrm{k}_{\mathrm{F}} \mathrm{x}\right) \cdot \cos (\mathrm{b} \varepsilon)-\mathrm{J}_{1}\left(2 \mathrm{k}_{\mathrm{F}} \mathrm{x}\right) \cdot \sin (\mathrm{b} \varepsilon) .
$$




\section{Anhang D: Analytische Bestimmung der Topografie}

Berechnet werden soll die Topografiespur $z(x)$ senkrecht $z u$ einer monatomaren Stufe bei $\mathrm{x}=0$. Hierzu wird der in Anhang B verwendete Tersoff-Hamann Formalismus mit den dort aufgeführten Bezeichnungen übernommen. Mit $\mathrm{U}_{\text {Bias }}$ sei $\mathrm{im}$ folgenden das für die Topografieregelung an die Probe gelegte Spannungspotential bezeichnet. $I_{\text {soll }}$ sei dementsprechend der Sollwert des Stromes bei der Topografieregelung.

\section{Topografie-Interpretation bei Potenziometriemessungen}

Im Potenziometriemodus liegt während der Topografieregelung an der Probe das Potenzial $\mathrm{U}_{\text {Bias }}$ und an der Spitze das Potenzial $\mathrm{U}_{\mathrm{Th}}(\mathrm{x})$ an. Für den Tunnelstrom gilt dann im Falle $\mathrm{U}_{\text {Bias }} \ll \mathrm{k}_{\mathrm{B}} \mathrm{T} / \mathrm{e}$ nach Gl. (B.1) bis (B.5)

$$
\mathrm{I}_{\text {soll }} \propto \int_{-\infty}^{\infty} \rho_{\mathrm{S}}(\varepsilon) \cdot \rho_{\mathrm{P}}(\overrightarrow{\mathrm{r}}, \varepsilon) \cdot\left(\Delta \mathrm{f}_{\mathrm{T}}(\varepsilon)+\overline{\mathrm{f}^{\prime}(\varepsilon)} \cdot \mathrm{e}\left(\mathrm{U}_{\mathrm{Th}}(\mathrm{x})-\mathrm{U}_{\text {Bias }}\right)\right) \cdot \mathrm{d} \varepsilon
$$

mit

$$
\begin{aligned}
& \Delta \mathrm{f}_{\mathrm{T}}(\varepsilon)=\mathrm{f}\left(\varepsilon, \mathrm{T}_{\mathrm{S}}\right)-\mathrm{f}\left(\varepsilon, \mathrm{T}_{\mathrm{P}}\right) \quad \text { und } \\
& \overline{\mathrm{f}^{\prime}(\varepsilon)}=\frac{1}{2} \cdot\left(\frac{\partial \mathrm{f}\left(\varepsilon, \mathrm{T}_{\mathrm{S}}\right)}{\partial \varepsilon}+\frac{\partial \mathrm{f}\left(\varepsilon, \mathrm{T}_{\mathrm{P}}\right)}{\partial \varepsilon}\right) .
\end{aligned}
$$

Gleichung (D.1) weiter aufgelöst ergibt

$$
\begin{aligned}
& \mathrm{I}_{\text {soll }} \propto \int_{-\infty}^{\infty} \rho_{\mathrm{S}}(\varepsilon) \cdot \rho_{\mathrm{P}}(\overrightarrow{\mathrm{r}}, \varepsilon) \cdot\left(\Delta \mathrm{f}_{\mathrm{T}}(\varepsilon)+\overline{\mathrm{f}^{\prime}(\varepsilon)} \cdot \mathrm{eU}_{\mathrm{Th}}(\mathrm{x})\right) \cdot \mathrm{d} \varepsilon \\
& -\int_{-\infty}^{\infty} \rho_{\mathrm{S}}(\varepsilon) \cdot \rho_{\mathrm{P}}(\overrightarrow{\mathrm{r}}, \varepsilon) \cdot \overline{\mathrm{f}^{\prime}(\varepsilon)} \cdot \mathrm{eU}_{\text {Bias }} \cdot \mathrm{d} \varepsilon .
\end{aligned}
$$

Das erste Integral in (D.4) beschreibt nach Anhang B, Gleichung (B.2) den Ausgleich zwischen thermischen Diffusionsstrom und feldgetriebenen Strom und verschwindet somit. Es bleibt ein Integralausdruck übrig, welcher im Kern einen Tunnelkontakt ohne thermoelektrischen Effekt beschreibt:

$$
\mathrm{I}_{\text {soll }} \propto-\int_{-\infty}^{\infty} \rho_{\mathrm{S}}(\varepsilon) \cdot \rho_{\mathrm{P}}(\overrightarrow{\mathrm{r}}, \varepsilon) \cdot \overline{\mathrm{f}^{\prime}(\varepsilon)} \cdot \mathrm{eU}_{\text {Bias }} \cdot \mathrm{d} \varepsilon .
$$

\footnotetext{
${ }^{33}$ Für eine standardmäßig benutzte Tunnelspannung von $\mathrm{U}_{\mathrm{Bias}}=1 \mathrm{mV}$ entspricht dies der Bedingung $\mathrm{T} » 12 \mathrm{~K}$; die tiefsten experimentell benutzten Temperaturen liegen bei ca. $80 \mathrm{~K}$.
} 
Das Potenziometrieverfahren eliminiert also in der topografischen Abbildung den thermoelektrischen Effekt und bildet die Oberfläche im wesentlichen so ab, wie sie auch bei Temperaturgleichheit von Spitze und Probe aussehen würde.

Die LDOS $\rho_{\mathrm{P}}$ der Oberfläche ist in Gleichung (B.11) von Anhang B beschrieben. Zur Vereinfachung werde angenommen, dass die Volumenzustandsdichte konstant bezüglich der Energie $\varepsilon$ ist (d.h. es gelte $\rho_{\mathrm{V} 0}^{\prime}=0$ ) und Volumen- und Oberflächenzustände dieselbe Abklinglänge zur Vakuumseite hin besitzen:

$$
\rho_{\mathrm{P}}(\overrightarrow{\mathrm{r}}, \varepsilon)=\rho_{\mathrm{P}}(\mathrm{z}) \cdot\left[1-\mathrm{C} \cdot \mathrm{J}_{0}\left(2 \cdot \mathrm{k}_{\|}(\varepsilon) \cdot \mathrm{x}\right)\right] .
$$

Von N. D. Lang [Lan1-2] wird für das exponentielle Abklingen der LDOS in z-Richtung bei einer effektiven Barrierenhöhe $\phi$ ein Ausdruck

$$
\rho_{\mathrm{P}}(\mathrm{z}) \propto \exp \left(-2 \mathrm{z} \cdot \hbar^{-1} \cdot \sqrt{2 \cdot \mathrm{m} \cdot \phi}\right)=\exp (-\alpha \cdot \mathrm{z})
$$

gefunden. Wenn von der LDOS der z-Anteil absepariert wird und die Abklingkonstante $\alpha$ sowie die Zustandsdichte der Spitze als näherungsweise konstant bezüglich der Energie $\varepsilon$ angesehen werden, dann lässt sich (D.5) umformen zu

$$
\mathrm{I}_{\text {soll }} \propto \exp (-\alpha \cdot \mathrm{z}) \cdot \int_{-\infty}^{\infty}\left(1-\mathrm{C} \cdot \mathrm{J}_{0}\left(2 \mathrm{k}_{\|}(\varepsilon) \mathrm{x}\right)\right) \cdot \overline{\mathrm{f}^{\prime}(\varepsilon)} \cdot \mathrm{d} \varepsilon
$$

Das Integral in (D.8) ist in (B.14) bzw. (B.27) gelöst und ergibt

$$
\mathrm{I}_{\text {soll }} \propto-\exp (-\alpha \cdot \mathrm{z}) \cdot\left[1-\mathrm{C} \cdot \mathrm{J}_{0}\left(2 \mathrm{k}_{\mathrm{F}} \mathrm{x}\right) \cdot \mathrm{h}_{\mathrm{N}}(\mathrm{x})\right]
$$

mit der in (B.28) definierten Einhüllendenfunktion $\mathrm{h}_{\mathrm{N}}(\mathrm{x})$. Gleichung (D.9) lässt sich nach $\mathrm{z}$ auflösen:

$$
\mathrm{z}=\mathrm{z}_{0}+\frac{1}{\alpha} \cdot \ln \left(1-\mathrm{C} \cdot \mathrm{J}_{0}\left(2 \mathrm{k}_{\mathrm{F}} \mathrm{x}\right) \cdot \mathrm{h}_{\mathrm{N}}(\mathrm{x})\right)
$$

Hier ist $z_{0}$ die mittlere Höhe der Spitze, und der logarithmische Term berücksichtigt die Interferenzeffekte. Der einzige Unterschied zu den in der Literatur berechneten Topografien [Bür 1, Bür 4] ist in der Einhüllendenfunktion $\mathrm{h}_{\mathrm{N}}(\mathrm{x}) \mathrm{zu}$ finden, welche hier berücksichtigt, dass es in Spitze und Probe zwei verschiedene Temperaturen, also auch zwei verschiedene Fermi-Dirac-Abklinglängen gibt.

\section{Topografie-Interpretation ohne Potenziometriemessung}

Die Topografiespur von alleinigen Topografiemessungen lässt sich herleiten, indem in Gleichung (D.1) die im dortigen Modus am Tunnelkontakt anliegende Spannung $\left(\mathrm{U}_{\mathrm{Bias}}-\mathrm{U}_{\mathrm{Th}}\right)$ durch eine konstante Spannung $U_{\text {Bias }}$ substituiert wird. Nach analogen Umformungen ist Gleichung (D.5) zu ersetzen durch die Gleichung 
(D.11) $\quad \mathrm{I}_{\text {soll }} \propto-\int_{-\infty}^{\infty} \rho_{\mathrm{S}}(\varepsilon) \cdot \rho_{\mathrm{P}}(\overrightarrow{\mathrm{r}}, \varepsilon) \cdot \overline{\mathrm{f}^{\prime}(\varepsilon)} \cdot \mathrm{e}\left(\mathrm{U}_{\text {Bias }}-\mathrm{U}_{\mathrm{Th}}(\mathrm{x})\right) \cdot \mathrm{d} \varepsilon$.

Im Falle einer Temperaturdifferenz zwischen Spitze und Probe addiert sich also rechnerisch die Thermospannung $\mathrm{zu}$ der an den Tunnelkontakt angelegten Spannung. Der Topografiemodus wird also mit einer ortsabhängigen Tunnelspannung ausgeführt. Die weiteren Umformungen erfolgen analog zu den in Gleichung (D.6) bis (D.10) dargestellten und ergeben

(D.12) $\quad \mathrm{z}=\mathrm{z}_{0}+\frac{1}{\alpha} \cdot \ln \left(\frac{1-\mathrm{C} \cdot \mathrm{J}_{0}\left(2 \mathrm{k}_{\mathrm{F}} \mathrm{x}\right) \cdot \mathrm{h}_{\mathrm{N}}(\mathrm{x})}{\left|1-\mathrm{U}_{\mathrm{Th}}(\mathrm{x}) / \mathrm{U}_{\text {Bias }}\right|}\right)$. 


\section{Verzeichnis verwendeter Formelzeichen und Abkürzungen}
A
Modulationstiefe bei periodisch modulierten Stufen (Kap. 10)
ARPES Winkelaufgelöste Photoemissionsspektroskopie (engl. „Angelresolved Photoemission $\underline{\text { Spectroscopy“) }}$
$\mathrm{C} \quad \mathrm{C}=\mathrm{P} \cdot \mathrm{R}=$ Anteil des Interferenzterms der Oberflächenzustände an der gesamten LDOS; Gl. (B.11) Anhang B
c Lichtgeschwindigkeit; $\mathrm{c}=2.9979 \cdot 10^{8} \mathrm{~m} / \mathrm{s}$
dI/dU Rastertunnelspektroskopie, s. Kap. 2.3.2
$\mathrm{E}_{\mathrm{F}} \quad$ Fermienergie
$\mathrm{E}_{\Gamma} \quad$ Energie der Bandunterkante der Oberflächenzustände; Gl. (6.1)
e Elementarladung; e $=1.6022 \cdot 10^{-19} \mathrm{As}$
e-e; e-ph Elektron-Elektron; Elektron-Phonon
$\mathrm{f}(\varepsilon, \mathrm{T}) \quad$ Fermifunktion; s. Gl (2.1)

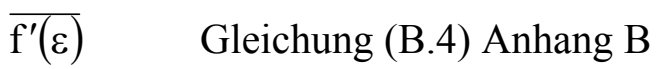
$\Delta \mathrm{f}_{\mathrm{T}}(\varepsilon) \quad$ Gleichung (B.5) Anhang B
$\mathrm{h}_{\mathrm{N}}(\mathrm{x}) \quad$ Nenner-Hüllfunktion der Thermospannung; s. Gl. (B.28) in Anhang B
$\mathrm{h}_{\mathrm{Z}}(\mathrm{x}) \quad$ Zähler-Hüllfunktionn der Thermospannung; s. Gl. (B.22) in Anhang B
$\mathrm{I}_{\mathrm{T}} \quad$ Tunnelstrom; s. Gl. (2.3)
i Komplexer Wert von $\sqrt{-1}$
$\mathrm{J}_{\mathrm{n}}(\mathrm{x}) \quad$ Besselfunktion erster Gattung n-ter Ordnung; s. Ref. [Bro, Wat]
k Wellenzahl
$\mathrm{k}_{\mathrm{B}} \quad$ Boltzmannkonstante; $\mathrm{k}_{\mathrm{B}}=1.3807 \cdot 10^{-23} \mathrm{~J} / \mathrm{K}=8.6175 \cdot 10^{-5} \mathrm{eV} / \mathrm{K}$
$\overrightarrow{\mathrm{k}}_{\|} \quad$ Wellenvektor $\overrightarrow{\mathrm{k}}_{\|}=\left(\mathrm{k}_{\mathrm{x}}, \mathrm{k}_{\mathrm{y}}, 0\right)$ parallel zur Oberfläche; s. Kap. 6
$\mathrm{k}_{\mathrm{F}} \quad$ Fermi-Wellenzahl der Oberflächenzustände; $\mathrm{k}_{\mathrm{F}}=\sqrt{2 \mathrm{~m}^{*} \varepsilon_{\mathrm{F}}} / \hbar$
KRIPES k-Aufgelöste inverse Photoemissions-Spektroskopie (engl. ,k-k-resolved inverse Photoemission $\underline{\text { Spectroscopy“c) }}$

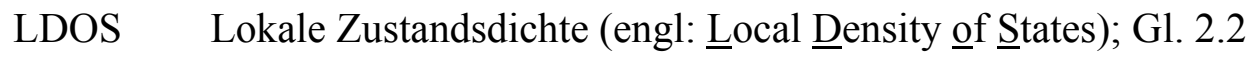
LHe Flüssiges Helium He (engl. Liquid Helium)
$\mathrm{L}_{\varphi}(\varepsilon, \mathrm{T}) \quad$ Kohärenzlänge eines Quasiteilchens; $\mathrm{L}_{\varphi}=\mathrm{v} \cdot \tau_{\varphi} ; \mathrm{Gl.}(8.9)$ 


$\begin{array}{ll}\mathrm{LN}_{2} & \text { Flüssiger Stickstoff } \mathrm{N}_{2} \text { (engl. Liquid Nitrogen) } \\ \mathrm{m}_{\mathrm{e}} & \text { Ruhemasse des Elektrons; } \mathrm{m}_{\mathrm{e}}=9.10953 \cdot 10^{-31} \mathrm{~kg} \\ \mathrm{~m}_{\mathrm{e}}^{*} & \text { Effektive Elektronenmasse; Tab. 6.1 } \\ \mathrm{P} & \text { Anteil der Oberflächenzustände an der gesamten LDOS; Gl. (B.10) Anhang B } \\ \mathrm{p} & \text { Periodizität der Modulation von Stufen (s. Kap. 10 } \\ \mathrm{p}-\mathrm{p} & \text { Peak-to-Peak-Wert } \\ \mathrm{R} & \text { Amplituden-Reflexionsgrad einer monatomaren Stufe (s. Anhang A) } \\ \overrightarrow{\mathrm{r}} & \text { Ortsvektor } \overrightarrow{\mathrm{r}}=(\mathrm{x}, \mathrm{y}, \mathrm{z}) \\ \mathrm{S}(\mathrm{T}) & \text { Thermokraft; } \mathrm{S}=\lim _{\Delta \mathrm{T} \rightarrow 0}\left(\mathrm{U}_{\mathrm{Th}} / \Delta \mathrm{T}\right) ; \text { Gl. (3.2) }\end{array}$

SThVM Raster-Thermospannungs-Mikroskopie (engl. Scanning Thermovoltage Microscopy); s. Kap. 3.3

STM Rastertunnelmikroskop (engl. „Scanning Tunneling Microscope“); s. Kap. 2

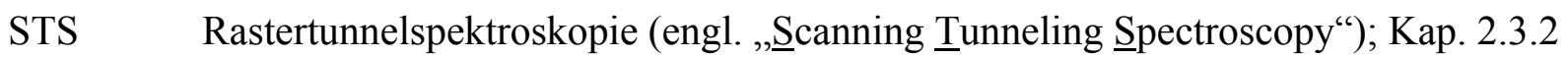

$\mathrm{S}_{\mathrm{xy}} \quad$ Normierungskonstante für Wellenfunktionen nach Integration entlang der Oberfläche (xy-Ebene); s. Gl. (7.1)

$\mathrm{S}_{\mathrm{S}}, \mathrm{S}_{\mathrm{P}} \quad \mathrm{s}_{\mathrm{S}}=\pi \cdot \mathrm{k}_{\mathrm{F}} \mathrm{x} \cdot \mathrm{k}_{\mathrm{B}} \mathrm{T}_{\mathrm{S}} / \varepsilon_{\mathrm{F}} \quad ; \quad \mathrm{s}_{\mathrm{P}}=\pi \cdot \mathrm{k}_{\mathrm{F}} \mathrm{x} \cdot \mathrm{k}_{\mathrm{B}} \mathrm{T}_{\mathrm{P}} / \varepsilon_{\mathrm{F}} ; \mathrm{s}$. Gl. (B.23) (B.24)

T Temperatur

$\Delta \mathrm{T} \quad$ Temperaturdifferenz zwischen Spitze und Probe, $\Delta \mathrm{T}=\mathrm{T}_{\mathrm{S}}-\mathrm{T}_{\mathrm{P}}$

$\mathrm{T}_{\mathrm{m}} \quad$ Mittlere Temperatur von Spitze und Probe, $\mathrm{T}_{\mathrm{m}}=\frac{1}{2} \cdot\left(\mathrm{T}_{\mathrm{P}}+\mathrm{T}_{\mathrm{S}}\right)$

$\mathrm{T}_{\min }, \mathrm{T}_{\max }$ Die jeweils kleinere bzw. größere der Temperaturen von Spitze und Probe

$\mathrm{T}_{\mathrm{P}}, \mathrm{T}_{\mathrm{S}} \quad$ Temperatur der Probe bzw. der Spitze

$\mathrm{U}_{\mathrm{T}} \quad$ Spannungsdifferenz am Tunnelkontakt; $\mathrm{U}_{\mathrm{T}}=\mathrm{U}_{\text {Spitze }}-\mathrm{U}_{\text {Probe }}$

$\mathrm{U}_{\text {Bias }} \quad$ Spannungspotenzial der Probe bei der Topografieregelung

$U_{\text {env }} \quad$ Einhüllende einer räumlich oszillierenden Thermospannung; s. Gl. (8.3)

UHV ㄴtra- $\underline{\text { Hoch-Vakuum }}$

$\mathrm{U}_{\text {Probe }} \quad$ Spannungspotenzial der Probe

$\mathrm{U}_{\mathrm{R}} \quad$ Rauschamplitude

$\mathrm{U}_{\text {Spitze }} \quad$ Spannungspotenzial der Spitze

$\mathrm{U}_{\mathrm{Th}} \quad$ Thermospannung; s. Kap. 3

v Geschwindigkeit eines Quasiteilchens; s. Kap. 8.3

$\mathrm{x} \quad \mathrm{x}$-Koordinate in der Oberflächenebene (ggf. Abstand zu einer Stufe; s. Abb. 7.1)

$\mathrm{x}_{\mathrm{m}} \quad$ Abstand des Einhüllendenmaximums in $\mathrm{U}_{\mathrm{Th}}$ von der Stufe; s. Kap. 8.2.3 c 
y $\quad$-Koordinate in der Oberflächenebene (ggf. parallel zur Stufenkante; s. Abb. 7.1

z Koordinate senkrecht zur Oberfläche (s. Abb. 7.1)

$\alpha \quad$ Abklingkonstante der Wellenfunktionen außerhalb des Kristalls; s. Gl. (D.7)

$\delta\left(\varepsilon-\varepsilon_{0}\right) \quad$ Distributionsfunktion; $\int \mathrm{f}(\varepsilon) \cdot \delta\left(\varepsilon-\varepsilon_{0}\right) \cdot \mathrm{d} \varepsilon=\mathrm{f}\left(\varepsilon_{0}\right)$

$\varepsilon \quad$ Energie relativ zum elektrochemischen Potenzial $\mu_{\mathrm{ec}}$ der Probe; s. Kap. 2.2

$\varepsilon_{\mathrm{F}} \quad$ Fermienergie der Oberflächenzustände; $\varepsilon_{\mathrm{F}}=\mu_{\mathrm{ec}}-\mathrm{E}_{\Gamma} ;$ s. Tab. 6.1

$\varepsilon\left(\mathrm{k}_{\|}\right) \quad$ Dispersionsrelation der Oberflächenzustände; $\varepsilon\left(\mathrm{k}_{\|}\right)=\hbar^{2} \mathrm{k}_{\|}^{2} /\left(2 \mathrm{~m}^{*}\right)-\varepsilon_{\mathrm{F}} ;$ s. Gl. (6.1)

$\hbar \quad$ Planck-Wirkungsquantum; $\hbar=\mathrm{h} / 2 \pi=1.054589 \cdot 10^{-34} \mathrm{Js}$

$\varphi \quad$ Phasenverschiebung einer Welle bei deren Reflexion; s. Anhang A

$\lambda_{\varepsilon} \quad$ Wellenlänge im Interferenzmuster; $\lambda_{\varepsilon}=\lambda / 2=\pi / \mathrm{k}_{\|} ; \mathrm{s}$. Gl. (B.40). s. Abb. 8.11

$\lambda_{\mathrm{e}-\mathrm{ph}} \quad$ Electron-Mass-Enhancement Parameter [Gri]: s. Gl. (8.16)

$\mu_{\mathrm{ec}} \quad$ Elektrochemisches Potenzial (der Probe); s. Kap. 2.2

$\pi \quad$ Kreiskonstante; $\pi=3.1415927$

$\rho_{\mathrm{OF}}(\overrightarrow{\mathrm{r}}, \varepsilon) \quad$ Lokale Zustandsdichte der Oberflächenzustände; s. Gl (A.11) Anhang A

$\rho_{\mathrm{P}}(\overrightarrow{\mathrm{r}}, \varepsilon) \quad$ Gesamte lokale Zustandsdichte der Probenoberfläche; s. Gl. (B.11) Anhang B

$\rho_{\mathrm{P} 0} \quad$ LDOS bei $\varepsilon=0$ für eine ungestörte Oberfläche; s. Gl. (B.10) Anhang B

$\rho_{\mathrm{S}}(\varepsilon) \quad$ Zustandsdichte der Spitze; s. Gl. (B.6) Anhang B

$\rho_{\mathrm{S} 0} \quad$ Zustandsdichte der Spitze bei $\varepsilon=0$; s. Gl. (B.6) Anhang B

$\rho_{\mathrm{S} 0}^{\prime} \quad$ Differentielle Zustandsdichte der Spitze bei $\varepsilon=0$; s. Gl. (B.6) Anhang B

$\rho_{\mathrm{V}}(\overrightarrow{\mathrm{r}}, \varepsilon) \quad$ Lokale Zustandsdichte der Volumenzustände; s. Gl. (B.7) Anhang B

$\rho_{\mathrm{v} 0} \quad$ Lokale Zustandsdichte der Volumenzustände bei $\varepsilon=0$; s. Gl. (B.7) Anhang B

$\rho_{\mathrm{V} 0}^{\prime} \quad$ Differentielle LDOS der Volumenzustände bei $\varepsilon=0$; s. Gl. (B.7) Anhang B

$\sigma_{\mathrm{e}}, \sigma_{\mathrm{T}} \quad$ Leitfähigkeiten im Volumenkristall bzw. über die Tunnelbarriere; s. Gl. (3.4)

$\tau_{\mathrm{e}}, \tau_{\mathrm{h}} \quad$ Lebensdauer angeregter Elektronen, G1. 8.11, 8.17, bzw. Löcher, Gl.(8.14, 8.18)

$\tau_{\mathrm{e}-\mathrm{e}}, \tau_{\mathrm{e}-\mathrm{ph}}$ Lebensdauer von Quasiteilchen für die Annahme von Elektron-Elektron-Streuung, Gl. (8.15), bzw. Elektron-Phonon-Streuung, Gl. (8.16)

$\tau_{\varphi} \quad$ Lebensdauer eines Quasiteilchens; s. Kap. 8.3

$\omega \quad$ Phononenfrequenz; s. Kap. 8.3

$\omega_{\mathrm{D}} \quad$ Debyefrequenz; s. Kap. 8.3

$\psi(\overrightarrow{\mathrm{r}}) \quad$ Wellenfunktion; s. Kap. 7

$\psi(\mathrm{z}) \quad$ Eindimensionale Wellenfunktion für die Richtung senkrecht zur Oberfläche;Kap.7 


\section{Literaturverzeichnis}

[Alb] O. Albrektsen, H. W. M. Salemink, K. A. Mørch und A. R. Thölen, Reliable tip preparation for highresolution scanning tunneling microscopy, Journal of Vacuum Science and Technology B 12, 3187 (1994)

[Ash] N. W. Ashcroft und N. D. Mermin, Solid State Physics, Saunders College Editions (1981)

[Avo 1] P. Avouris und Y. W. Lyo, Observation of quantum-size effects at room temperature on metal surfaces with STM, Science 264 (5161), 942 (1994)

[Avo 2] Ph. Avouris, I.-W. Lyo, R.W. Walkup und Y. Hasegawa, Real space imaging of electron scattering phenomena at metal surfaces, J. Vac. Sci. Technol. B 12, 447 (1994)

[Avo 3] Phaedon Avouris, Studies of confined states and quantum size effects with scanning tunneling microscopy, Solid State Communications 92, 11 (1994)

[Avo 4] Ph. Avouris, I. W. Lyo und Y. Hasegawa, Probing electrical transport, electron interference, and quantum size effects at surfaces wih STM/STS, IBM Journal of Research and Development 39, 603 (1995)

[Avo 5] P. Avouris, I. W. Lyo und M. P Molinas, STM studies of the interaction of surface state electrons on metals with steps and adsorbates, Chemical Physics Letters 240, 423 (1995)

[Bar 1] J. Bardeen, Tunneling from a many-particle point of view, Physical Review Letters 6, 57 (1961)

[Bar 2] R. D. Barnard, Thermoelectricity in Metals and Alloys, Taylor and Francis (London 1972)

[Bar 3] J. Bardeen, Surface States and Rectification at a Metal Semi-Conductor Contact, Physical Review 71, 717 (1947)

[Bar 4] J. V. Barth, H. Brune, G. Ertl und R. J. Behm, STM observations on the reconstructed Au(111) surface: Atomic structure, long-range superstructure, rotational domains, and surface defects, Physical Review B 42, 9307 (1990)

[Bec] A. C. Becquerel, Annales de chimie physique 23, 135 (1823) und 31, 357 (1826)

[Ber] R. Berndt, L. Li, W. D. Schneider und S. Crampin, Surface-state linewidth from scanning tunnelling spectroscopy, Applied Physics A69, 503 (1999)

[Bes 1] F. Besenbacher, Scanning tunnelling microscopy studies of metal surfaces, Reports on Progress in Physics 59, 1737 (1996)

[Bes 2] K. Besocke, An easily operable scanning tunneling microscope, Surface Science 181, 145 (1987)

[Bes 3] J. Frohn, J. F. Wolf, K. Besocke und M. Teske, Coarse tip distance adjustment and positioner for a scanning tunneling microscope, Review of Scientific Instruments 60, 1200 (1989)

[Bin 1] G. Binnig und H. Rohrer, Scanning tunnelling microscopy, Helvetica Physica Acta 55, 726 (1982)

[Bin 2] G. Binning, H. Rohrer, Ch. Gerber und E. Weibel, Surface Studies by Scanning Tunneling Microscopy, Physical Review Letters 49, 57 (1982)

[Bin 3] G. Binnig und H. Rohrer, In touch with atoms, Reviews of Modern Physics 71, S324 (1999)

[Bra] W. H. Brattain und W. Shockley, Density of Surface States on Silicon Deduced from Contact Potential Measurements, Physical Review 72, 345 (1947)

[Bri 1] B. G. Briner, Ph. Hofmann, M. Doering, H.-P. Rust, E. W. Plummer und A. M. Bradshaw, Observation of interfering Bloch waves, Europhysics Letters 39, 67 (1997)

[Bri 2] B. G. Briner, P. Hofmann, M. Doering, H.-P. Rust, A. M. Bradshaw, L. Petersen, P. Sprunger, E. Laegsgaard, F. Besenbacher und E. W. Plummer, Looking at electronic wave functions on metal surfaces, Europhysics news 28, 148 (1997)

[Bri 3] B. G. Briner, Ph. Hofmann, M. Doering, H.-P. Rust, E. W. Plummer und A. M. Bradshaw, Chargedensity oscillations on Be(10-10): Screening in a non-free two-dimensional electron gas, Physical Review 58, 13931 (1998)

[Bro] I. N. Bronstein und K. A. Semendjajew, Taschenbuch der Mathematik, 20. Aufl., Verlag Harri Deutsch (Frankfurt 1981) 
[Buc] M. J. Buckingham, Noise in electronic devices and systems, Wiley (Chichester 1983)

[Bür 1] Lukas Bürgi, Scanning Tunneling Microscopy as Local Probe of Electron Density, Dynamics, and Transport at Metal Surfaces, Dissertationsarbeit, Département de Physique, École Polytechnique Fédérale de Lausanne (Gruppe K. Kern, 1999)

[Bür 2] L. Bürgi, O. Jeandupeux, A. Hirstein, H. Brune und K. Kern, Confinement of Surface State Electrons in Fabry-Pérot Resonators, Physical Review Letters, 81, 5370 (1998)

[Bür 3] L. Bürgi, O. Jeandupeux, H. Brune und K. Kern, Probing Hot-Electron Dynamics at Surfaces with a Cold Scanning Tunneling Microscope, Physical Review Letters 82, 4516 (1999)

[Bür 4] L. Bürgi, H. Brune, O. Jeandupeux und K. Kern, Quantum coherence and lifetimes of surface-state electrons, Journal of Electron Spectroscopy and Related Phenomena 109, 33 (2000)

[Bür 5] L. Bürgi, L. Petersen, H. Brune und K. Kern, Noble metal surface states: deviations from parabolic dispersion, Surface Science 447, L157 (2000)

[Che 1] W. Chen, V. Madhavan, T. Jamneala und M. F. Crommie, Scanning Tunneling Microscopy Observation of an Electronic Superlattice at the Surface of Clean Gold, Physical Review Letters 80, 1469 (1998)

[Che 2] D. Chen, Electron fringes and standing wave states in a quantum wedge, Journal of Electron Spectroscopy and Related Phenomena 109, 85 (2000)

[Che 3] C. J. Chen, Introduction to Scanning Tunneling Microscopy, Oxford Series in Optical and Imaging Sciences, Oxford University Press (Oxford 1993)

[Cro 1] M. F. Crommie, C. P. Lutz und D. M. Eigler, Imaging standing waves in a two-dimensional electron gas, Nature 363, 524 (1993)

[Cro 2] M. F. Crommie, C. P. Lutz und D. M. Eigler, Confinement of Electrons to Quantum Corrals on a Metal Surface, Science 262, 218 (1993)

[Cro 3] M. F. Crommie, C. P. Lutz, D. M. Eigler und E. J. Heller, Quantum corrals, Physica D 83, 98 (1995)

[Cro 4] M. F. Crommie, C. P. Lutz, D. M. Eigler und E. J. Heller, Quantum interference in 2D atomic-scale structures, Surface Science 361/362, 864 (1996)

[Cro 5] M. F. Crommie, Observing electronic scattering in atomic-scale structures on metals, Journal of Electron Spectroscopy and Related Phenomena 109, 1 (2000)

[Dat] S. Datta, Electronic Transport in Mesoscopic Systems, Cambridge Studies in Semiconductor Physics and Microelectronic Engieering 3, Cambridge University Press (Cambridge 1995)

[Dav 1] S. G. Davison und M. Steslicka, Basic theory of surface states, Monographs on the physics and chemistry of materials No. 46, Clarendon Press (Oxford 1996)

[Dav 2] S. G. Davison und J. D. Levine, Surface States, Solid State Physics, Editors:, Clarendon Press (Oxford 1996),

[deB] L. de Broglie, A. Barur, G Lochak und A. van der Merwe, Heisenberg's uncertainties and the probabilistic interpretation of wave mechanics : with critical notes of the author, Kluwer (Dordrecht 1990)

[Des] M.C. Desjonquères und D. Spanjaard, Concepts in Surface Physics, $2^{\text {nd }}$ Ed., Springer (Berlin 1996)

[DI] Nanoscope IIIa Multimode Scanning Probe Microscope, Digital Instruments GmbH, Janderstr. 9, D68199 Mannheim, Tel. 49(0)621-842100, www.digmbh.de

[Dra] K. Dransfeld und J. Xu, The heat transfer between a heated tip and a substrate: fast thermal microscopy, Journal of Microscopy 152, 35 (1988)

[Eng 1] K. J. Engel, M. Wenderoth, N. Quaas, T. C. G. Reusch, K. Sauthoff und R. G. Ulbrich, Thermovoltage mapping of standing electron waves on Au(111) surfaces at low temperatures, Physical Review B 63, $165402(2001)$

[Eng 2] K. J. Engel, Thermoelektrische Kontrasteffekte in der Rastertunnelpotentiometrie, Diplomarbeit, IV. Physikalisches Institut der Universität Göttingen (Gruppe R. G. Ulbrich, 1996)

[Feu] B. Feuerbacher, B. Fitton und R. F. Willis, Photoemission and the Electronic Properties of surfaces, John Wiley (Chichester, 1978)

[Fli] T. Fließbach, Quantenmechanik, 3. Aufl., Spektrum Akademischer Verlag (Heidelberg, 2000)

[Fuj 1] D. Fujita, K. Amemiya, T. Yakabe und H. Nejoh, Anisotropic Standing- Wave Formation on an Au(111)- (23 x sqrt(3)) Reconstructed Surface, Physical Review Letters 78, 3904 (1997) 
[Fuj 2] D. Fujita, K. Amemiya, T. Yakabe, H. Nejoh, T. Sato und M. Iwatsuki, Possible existence of quasi onedimensional propagation of electron waves on a reconstructed Au(111) surface, Surface Science 386, 315 (1997)

[Fuj 3] D. Fujita, K. Amemiya, T. Yakabe, H. Nejoh, T. Sato und M. Iwatsuki, Effect of herringbone reconstruction on interference of two-dimensional electron gas on Au(111) surfaces, Journal of Vacuum Science \& Technology A 15, 1613 (1997)

[Fuj 4] D. Fujita, K. Amemiya, T. Yakabe, H. Nejoh, T. Sato und M. Iwatsuki, Observation of two-dimensional Fermi contour of a reconstructed Au(111) surface using Fourier transform scanning tunneling microscopy, Surface Science 423, 160 (1999)

[Fuj 5] D. Fujita, Reply to Comment on Ref. [Pet 3], Surface Science 443, 157 (1999)

[Gar] P. O. Gartland und B. J. Slagsvold, Transitions conserving parallel momentum in photoemission from the (111) face of copper, Physical Review B 12, 4047 (1975)

[Geb] T. H. Geballe und G. F. Giauque, Journal of the American Chemical Society 74, 2368 (1952)

[Gia] I. Giaever, Energy Gap in Superconductors Measured by Electron Tunneling, Physical Review Letters 5,147 (1960)

[Gol 1] A. Goldmann und R. Matzdorf, Surface States on Metals, Ed. Mac Donald, Taglauer, Wandelt, Surface Science: Principles and current Applications, Springer (1996)

[Gol 2] A. Goldmann, V. Dose und G. Borstel, Empty electronic states at the (100), (110), and (111) surfaces of nickel, copper, and silver, Physical Review B 32, 1971 (1985)

[Gra] I. S. Gradshteyn, I. M. Ryzhik und A. Jeffrey, Table of integrals, series, and products, 5.ed., Academic Press (Boston 1995)

[Gri] G. Grimvall, The Electron-Phonon Interaction in Metals, Ed. E.P. Wohlfahrt, North-Holland Publishing Company (Amsterdam 1981)

[Haa] P. Haasen und J. Mordike, Physical Metallurgy, $3^{\text {rd }}$ ed., Cambridge University Press (Cambridge 1996); deutsch: Physikalische Metallkunde, 3. Aufl., Springer Verlag (Berlin 1994)

[Han] K. Højrup Hansen, J. Gottschalck, L. Petersen, B. Hammer, E. Lægsgaard, F. Besenbacher und I. Stensgaard, Surface waves on NiAl(110), Physical Review B 63, 115421 (2001)

[Har] U. Harten, A. M. Lahee, J. Peter Toennies und Ch. Wöll, Observation of a soliton reconstruction of Au(111) by high-resolution helium-atom diffraction, Physical Review Letters 54, 2619 (1985)

[Has 1] Y. Hasegawa und Ph. Avouris, Direct Observation of Standing Wave Formation at Surface Steps Using Scanning Tunneling Spectroscopy, Physical Review Letters 71, 1071 (1993)

[Has 2] Y. Hasegawa und P. Avouris, Real space observation of standing waves at metal surfaces and the determination of surface state dispersion with the scanning tunneling microscope, Japanese Journal of Applied Physics, Part 1, 33, no.6B, 3675 (1994)

[Has 3] Y. Hasegawa, I. W. Lyo und P. Avouris, STM study on two-dimensional electronic system localized on surfaces, Science Reports of the Research Institutes, Tohoku University, Series A, 44, no.1, 99 (1997)

[Hel 1] E. J. Heller, M. F. Crommie, C. P. Lutz und D. M. Eigler, Scattering and Absorption of Surface Electron Waves in Quantum Corrals, Nature 369, 464 (1994)

[Hel 2] E. J. Heller, M. F. Crommie, C. P. Lutz und D. M. Eigler, Mesoscopic systems and quantum corrals, AIP Conference Proceedings 360, 3 (1995), XIX ICPEAC, The physics of electronic and atomic collisions

[Hel 3] E. J. Heller, Bound-State Eigenfunctions of Classically Chaotic Hamiltonian Systems: Scars of Periodic Orbits, Physical Review Letters 53, 1515 (1984)

[Hel 4] E. J. Heller, Electrons in the looking glass, Nature 403, 489 (2000)

[Hel 5] K.-H. Hellwege, Einführung in die Festkörperphysik, 3. Auflage, Springer-Verlag (Berlin 1994)

[Hör 1] G. Hörmandinger, Imaging of the Cu(111) surface state in scanning tunneling microscopy, Physical Review B 49, 13897 (1994)

[Hör 2] G. Hörmandinger, Comment on Ref. [Has 1]] Physical Review Letters 73, 910 (1994)

[Höv] H. Hövel, B. Grimm und B. Reihl, Modification of the Shockley-type surface state on Ag(111) by an adsorbed xenon layer, Surface Science 477, 43 (2001) 
[Hof 1] P. Hofmann, B. G. Briner, M. Doering, H.-P. Rust, E. W. Plummer und A. M. Bradshaw, Anisotropic Two-Dimensional Friedel Oscillations, Physical Review Letters 79, 265 (1997)

[Hof 2] D. Hoffmann, Thermospannung in der Rastertunnel- Mikroskopie: Untersuchung an homogenen und heterogenen Metalloberflächen, Dissertationsarbeit, Universität Stuttgart (Gruppe R. Möller, 1998)

[Hof 3] D. Hoffmann, J. Y. Grand, R. Möller, A. Rettenberger und K. Läuger, Thermovoltage across a vacuum barrier investigated by scanning tunneling microscopy: Imaging of standing waves, Physical Review B. 52, 13796 (1995)

[Hof 4] D. Hoffmann, A. Haas, T. Kunstmann, J. Seifritz und R. Möller, Thermovoltage in Scanning Tunneling Microscopy, Journal of Vacuum Science and Technology A 15, 1418 (1997)

[Hof 5] D. Hoffmann, J. Seifritz, B. Weyers und R. Möller, Thermovoltage in scanning tunneling microscopy, Journal of Electron Spectroscopy and Related Phenomena 109, 117 (2000)

[Hof 6] D. H. Hoffmann, A. Rettenberger, J.-Y. Grand, K. Läuger, P. Leiderer, K. Dransfeld und R. Möller, Thermovoltages in vacuum tunneling investigated by scanning tunneling microscopy, Thin Solid films 264, 223 (1995)

[Hul 1] S. L. Hulbert, P. D. Johnson, N. G. Stoffel, W. A. Royer und N. V. Smith, Crystal-induced and imagepotential-induced empty surface states on $\mathrm{Cu}(111)$ and $\mathrm{Cu}(001)$, Physical Review B 31, 6815 (1985)

[Hul 2] S. L. Hulbert, P. D. Johnson, N. G. Stoffel und N. V. Smith, Unoccupied bulk and surface states on Ag(111) studied by inverse photoemission, Physical Review B 32, 3451 (1985)

[Iba] H. Ibach und H. Lüth, Solid-state physics : an introduction to principles of materials science, 2. Ed., Springer (Berlin 1996); deutsche Übersetzung: Festkörperphysik : Einführung in die Grundlagen, 5. Aufl., Springer (Berlin 1999)

[Jea] O. Jeandupeux, L. Bürgi, A. Hirstein, H. Brune und K. Kern, Thermal damping of quantum interference patterns of surface-state electrons, Physical Review B 59, 15926 (1999)

[Jep] O. Jepsen, D. Glötzel und A. R. Mackintosh, Potentials, band structures, and Fermi surfaces in the noble metals, Physical Review B 23, 2684 (1981)

[Joh] J. B. Johnsson, Thermal agitation of electricity in conductors, Nature 119, 50; Physical Review 29, 367 (1927); Physical Review 32, 97 (1928)

[Kev] S. D. Kevan und R. H. Gaylord, High-resolution photoemission study of the electronic structure of the noble-metal (111) surfaces, Physical Review B 36, 5809 (1987)

[Kir 1] J. R.. Kirtley, S. Washburn und M. J. Brady, Direct Measurement of Potential Steps at Grain Boundaries in the Presence of Current Flow, Physical Review Letters 60, 1546 (1988)

[Kir 2] J. R. Kirtley, S. Washburn und M. J. Brady, Scanning Tunneling Measurements of Potential Steps at Grain Boundaries in the Presence of Current Flow, IBM Journal of Research and Development 32, 414 (1988)

[Kli] J. Kliewer, R. Berndt, E. V. Chulkov, V. M. Silkin, P. M. Echenique und S. Crampin, Dimensionality Effects in the Lifetime of Surface States, Science 288 (Iss. 5470), 1399 (2000)

[Koh] M. Kohler, Thermischer Kontaktwiderstand von Metallen und der dadurch verursachte Temperatursprung im Kontakt. Neuartige Thermokräfte im einmetallischen Kreis und ihr Zusammenhang mit dem Benedickseffekt, Annalen der Physik 38, 542 (1940)

[Kro] R. de L. Kronig und W. G. Penney, Proceedings of the Royal Society of London A 130, 499 (1931)

[Lan 1] N. D. Lang, Spectroscopy of single atoms in the scanning-tunneling microscope, Physical Review B 34, $5947(1986)$

[Lan 2] N. D. Lang, Apparent barrier height in scanning tunneling microscopy, Physical Review B 37, 10395 (1988)

[LaS] S. LaShell, B. A. Mc Dougall und E. Jensen, Spin Splitting of an Au(111) Surface State Band Observed with Angle Resolved Photoelectron Spectroscopy, Physical Review Letters 77, 3419 (1996)

[Lea] C. R. Leavens und G. C. Aers, Vacuum Tunneling Thermopower: Normal Metal Electrodes, Solid State Communications 61, 289 (1987)

[Len] B. Lengeler, W. R. Wampler, R. R. Bourassa, K. Mika, K. Wingerath und W. Uelhoff, Precision measurements of cyclotron masses and Fermi velocities in the noble metals by the de Haas-van Alphen effect, Physical Review B 15, 5493 (1977) 
[Li 1] J. Li, W. D. Schneider und R. Berndt, Local Density of States from spectroscopic scanning tunneling microscope images: $\mathrm{Ag}$ (111), Physical Review B 56, 7656 (1997)

[Li 2] J. Li, W. D. Schneider und R. Berndt, Analysis of spectroscopic scanning tunneling microscope images, Applied Physics A 66, S167 (1998)

[Li 3] J. Li and W. D. Schneider, R. Berndt und S. Crampin, Electron Confinement to Nanoscale Ag Islands on Ag(111): A Quantitative Study, Physical Review Letters 80, 3332 (1998)

[Li 4] J. Li, W. D. Schneider, S. Crampin und R. Berndt, Tunnelling spectroscopy of surface state scattering and confinement, Surface Science 422, 95 (1999)

[Li 5] J. Li, W. D. Schneider, R. Berndt, O. R. Bryant und S. Crampin, Surface-State Lifetime Measured by Scanning Tunneling Spectroscopy, Physical Review Letters 81, 4464 (1998)

[Liu] S. H. Liu, C. Hinnen, C. Nguyen Van Huong, N. R. De Tacconi und K. M. Ho, Journal of electroanalytical chemistry and interfacial electrochemistry 176, 325 (1984)

[Lüt] H. Lüth, Surfaces and Interfaces of Solid Materials, Third Edition, Springer-Verlag (Berlin 1995)

[Lyn] J. W. Lynn, H. G. Smith und R. M. Nicklow, Lattice Dynamics of Gold, Physical Review B 8, 3493 (1973)

[Mae] A. Maeland und T. B. Flanagan, Canadian journal of physics 42, 2364 (1964)

[Maj] A. Majumdar, J. P. Carrejo und J. Lai, Thermal imaging using the atomic force microscope, Applied Physics Letters 62, 2501 (1993)

[Man] H. C. Manoharan, C. P Lutz und D. M. Eigler, Quantum mirages formed by coherent projection of electronic structure, Nature 403 (6769), 512 (2000)

[Mar 1] D. L. Martin, Anomalous Low-Temperature Specific Heat of Gold, Physical Review Letters 12, 723 (1964)

[Mar 2] D. L. Martin, Specific Heats of Copper, Silver, and Gold below $30^{\circ} \mathrm{K}$, Physical Review 141, 576 (1966)

[Mar 3] D. L. Martin, Specific Heats below $3^{\circ} \mathrm{K}$ of Pure Copper, Silver, and Gold, and of Extremely Dilute Gold-Transition-Metal Alloys, Physical Review 170, 650 (1968)

[MaT] H. Schlich, MaTeck GmbH, Karl-Heinz-Beckurts-Str. 13, D-52428 Jülich, Tel. 49(0)2461-690740, www.mateck.de

[Mat 2] R. Matzdorf, Investigation of line shapes and line intensities by high-resolution UV-photoemission spectroscopy. Some case studies on noble-metal surfaces, Surface Science Reports 30, 153 (1998)

[McD] B. A. McDougall, T. Balasubramanian und E. Jensen, Phonon contribution to quasiparticle lifetimes in Cu measured by angle-resolved photoemission, Physical Review B 51, 13891 (1995)

[Mem] N. Memmel und E. Bertel, Elektronische Oberflächenzustände an Metallen - Ursache und Bedeutung, Physikalische Blätter 53, 323 (1997)

[Mer] L. Merz und H. Jaschek, Grundkurs der Regelungstechnik: Einführung in die praktischen und theoretischen Methoden, 13. Aufl., Oldenbourg (München 1996)

[Mey] W. E. Meyerhof, Contact Potential Difference in Silicon Crystal Rectifiers, Physical Review 71, 727 (1947)

[Möl 1] R. Möller, A. Esslinger und B. Koslowski, Noise in vacuum tunneling: Application for a novel scanning microscope, Applied Physics Letters 55, 2360 (1989)

[Möl 2] R. Möller, A. Esslinger und B. Koslowski, Thermal noise in vacuum scanning tunneling microscopy at zero bias voltage, Journal of Vacuum Science and Technology A 8, 590 (1990)

[Möl 3] R. Möller, C. Baur, A. Esslinger und P. Kürz, Scanning noise potentiometry, Journal of Vacuum Science and Technology B 9, 609 (1991)

[Mor 1] M. Morgenstern, D. Haude, V. Gudmundsson, Chr. Wittneven, R. Dombrowski und R. Wiesendanger, Origin of Landau Oscillations observed in scanning tunneling spectroscopy on n-InAs(110), Physical Review B 62, 7257 (2000)

[Mor 2] M. Morgenstern, Chr. Wittneven, R. Dombrowski und R. Wiesendanger, Spatial fluctuations of the density of states in magnetic field observed with scanning tunneling microscopy, Physical Review Letters 84, 5588 (2000) 
[Mor 3] K. Morgenstern, E. Laegsgaard, I. Stensgaard, F. Besenbacher, M. Bohringer, W. D. Schneider, R. Berndt, F. Mauri, A. De Vita und R. Car, Stability of two-dimensional nanostructures, Applied Physics A69, 559 (1999)

[Mot] N. F. Mott und H. Jones, The Theory of the Properties of Metals and Alloys, Clarendon Press (Oxford 1936)

[Mur 1] P. Muralt und D. W. Pohl, Scanning tunneling potentiometry, Applied Physics Letters 48, 514 (1986)

[Mur2] P. Muralt, D.W. Pohl und W. Denk, Wide-range, low-operating voltage, bimorph STM: Application as a potentiometer, IBM Journal of Research and Development 30, 44 (1986)

[Nag] C. Nagl, O. Haller, E. Platzgummer, M. Schmid und P. Varga, Submonolayer growth of Pb on Cu(111): Surface alloying and de-alloying, Surface Science 321, 237 (1994)

[Nar] Shobhana Narasimhan und David Vanderbilt, Elastic stress domains and the herringbone reconstruction on $A$ u(111), Physical Review Letters 69, 1564 (1992)

[Nej] H. Nejoh, D. Fujita, T. Yakabe, A. Itakura und T. Uchihashi, Propagating electrons along domain walls of Au(111) observed by interference of coherent electrons at low temperature, Czechoslovak Journal of Physics 46, Suppl. S4, 2357 (1996)

[Nyq] H. Nyquist, Thermal agitation in conductors, Physical Review 29, 614 (1927); Physical Review 32,110 (1927)

[Non] M. Nonnenmacher und H. K. Wickramasinghe, Scanning probe microscopy of thermal conductivity and subsurface properties, Applied Physics Letters 61, 168 (1992)

[Noz] P. Nozières und D. Pines, The theory of quantum liquids, Advanced book classics, Perseus Books (Cambridge, 1999)

[Oes 1] E. Oesterschulze, L. Hadjiiski, M. Stopka und R. Kassing, Laser interferometry and SXM- techniques for thermal characterization of thin films, Materials Science Forum 185-188, 43 (1995)

[Oes 2] E. Oesterschulze und M. Stopka, Photothermal imaging by scanning thermal microscopy, Journal of Vacuum Science and Technology A 14, 1172 (1996)

[Oes 3] E. Oesterschulze, M. Stopka, L. Ackermann, W. Scholz und S. Werner, Thermal imaging of thin films by scanning thermal microscope, Journal of Vacuum Science and Technology B 14, 832 (1996)

[Ole] L. Olesen, M. Brandbyge, M. R. Sørensen, K. W. Jacobsen, E. Lægsgaard, I. Stensgaard und F. Besenbacher, Apparent Barrier Height in Scanning Tunneling Microscopy Revisited, Physical Review Letters 76,1485 (1996)

[Pan] R. Paniago, R. Matzdorf, G. Meister und A. Goldmann, Temperature dependence of Shockley-type surface energy bands on Cu(111), Ag(111) and Au(111), Surface Science 336, 113 (1995)

[Par] J. Y. Park, U. D. Ham, S. J. Kahng, Y. Kuk, K. Miyake, K. Hata und H. Shigekawa, Modification of surface-state dispersion upon Xe adsorption: A scanning tunneling microscope study, Physical Review B 62, R16341 (2000)

[Pas] D. W. Pashley, M. J. Stowell, M. H. Jacobs und T. J. Law, The Growth and Structure of Gold and Silver Deposits Formed by Evaporation Inside an Electron Microscope, Philosophical Magazine 10, 127 (1964)

[Pat] K. Patorski, The self-imaging phenomenon and ist applications, Progress in Optics 27, 1 (1989); Ed. E. Wolf, North-Holland (Amsterdam)

[Pel] J. P. Pelz und R.H. Koch, Extremly low-noise potentiometry with a scanning tunneling microscope, Review of Scientific Instruments 60, 301 (1989)

[Pet 1] L. Petersen, P. T. Sprunger, Ph. Hofmann, E. Lægsgaard, B. G. Briner, M. Doering, H.-P. Rust, A. M. Bradshaw, F. Besenbacher und E. W. Plummer, Direct imaging of the two-dimensional Fermi contour: Fourier-transform STM, Physical Review B 57, R6858 (1998)

[Pet 2] L. Petersen, P. Laitenberger, E. Lægsgaard und F. Besenbacher, Screening waves from steps and defects on Cu(111) and Au(111) imaged with STM: Contribution from bulk electrons, Physical Review B 58, 7361 (1998)

[Pet 3] L. Petersen, L. Bürgi, H. Brune, F. Besenbacher und K. Kern, Comment on Ref. [Fuj 4], Surface Science 443, 154 (1999)

[Pet 4] L. Petersen, Ph. Hofmann, E. W. Plummer und F. Besenbacher, Fourier Transform-STM: determining the surface Fermi contour, Journal of Electron Spectroscopy and Related Phenomena 109, 97 (2000) 
[Pet 5] P.T. Sprunger, L. Petersen, E.W. Plummer, E. Laegsgaard und F. Besenbacher, Giant Friedel oscillations on the beryllium(0001) surface, Science 275 (5307), 1764 (1997)

[Pet 6] L. Petersen und P. Hedegård, A simple tight-binding model of spin-orbit splitting of sp-derived surface states, Surface Science 459, 49 (2000)

[Qu] Z. Qu, L. Ye, A. Goonewardene, N. Mainkar, K. Subramanian, J. Karunamuni, R. L. Stockbauer und R. L. Kurtz, Imaging the surface state of Cu(111), Journal of Vacuum Science and Technology A 12, 2187 (1994)

[Qua] T. Quast, Topographie von Goldschichten auf Glimmersubstrat: Untersuchung mit dem Rastertunnelmikroskop, Diplomarbeit, IV. Physikalisches Institut der Universität Göttingen (Gruppe R. G. Ulbrich, 1995)

[Ray] Lord Rayleigh, Philosophical Magazine 11, 196 (1881)

[Rei 1] F. Reinert, G. Nicolay, S. Schmidt, D. Ehm und S. Hüfner, Direct measurements of the L-gap surface states on the (111) face of noble metals by photoelectron spectroscopy, Physical Review B 63, 115415 (2001)

[Rei 2] G. Reiss, L. E. Levine und D. A. Smith, Ultrahigh vacuum scanning-tunneling microscope for in situ studies of annealing and electromigration behavior of thin films, Journal of Vacuum Science and Technology B 11, 108 (1993)

[Rep] J. Repp, F. Moresco, G. Meyer, K. H. Rieder, P. Hyldgaard und M. Persson, Substrate Mediated LongRange Oscillatory Interaction between Adatoms: $\mathrm{Cu} / \mathrm{Cu}$ (111), Physical Review Letters 85, 2981 (2000)

[Ret] A. Rettenberger, C. Baur, K. Läuger, D. Hoffmann, J. Y. Grand und R. Möller, Variation of the thermovoltage across a vacuum tunneling barrier: copper islands on Ag(111), Applied Physics Letters 67, 1217 (1995)

[Roa] D. J. Roaf, Philosophical Transactions of the Royal Society 255, 85 (1962)

[Ros] M. A. Rosentreter, Rastertunnelmikroskopie auf der GaAs(110)-Oberfläche bei Temperaturen von 8 K300 K, Dissertation, IV. Physikalisches Institut der Universität Göttingen (Gruppe R. G. Ulbrich, 1997)

[Sch 1] M. A. Schneider, Rastertunnelmikroskop-Untersuchung der Streuung von Elektronen an Defekten am Beispiel dünner Au-Filme, Dissertation, IV. Physikalisches Institut der Universität Göttingen (Gruppe R. G. Ulbrich, 1997)

[Sch 2] M. A. Schneider, M. Wenderoth, K. J. Engel, M. A. Rosentreter, A. J. Heinrich und R. G. Ulbrich, Local electronic structure at steps on Au(111) investigated by the thermovoltage in scanning tunneling microscopy, Applied Physics A 66, S161 (1998)

[Sch 3] M. Schmid, S. Crampin und P. Varga, STM and STS of bulk electron scattering by subsurface objects, Journal of Electron Spectroscopy and Related Phenomena 109, 71 (2000)

[See 1] H. C. Ørsted, Notiz von neuen electrisch-magnetischen Versuchen des Herrn Seebeck in Berlin, Annalen der Physik 73, 430 (1823)

[See 2] T. J. Seebeck, Annalen der Physik 82, S. 1, fortgesetzt auf S. 133 und S. 253 (1826)

[Sei] J. Seifritz, Thermospannung in der Rastertunnelmikroskopie: Untersuchungen an homogenen und heterogenen Metall- und Halbleiteroberflächen, Dissertation, Institut für Laser und Plasmaphysik der Universität Essen (Gruppe R. Möller, 2000)

[Shk] O.I. Shklyarevskii, A. G. M. Jansen, J. G. H. Hermsen und P. Wyder, Thermoelectric Voltage between Identical Metals in Point-Contact Configuration, Physical Review Letters 57, 1374 (1986)

[Sho] W. Shockley und G. L. Pearson, On the Surface States Associated with a Periodical Potential, Physical Review 74, 232 (1948)

[Spe] Specs GmbH, Voltastr. 5, D-13355 Berlin, Tel. 030-4678240, www.specs.de

[Sto] J. A. Støvneng und P. Lipavský, Thermopower in scanning tunneling microscope experiments, Physical Review B 42, 9214 (1990)

[SW 1] Referenzenliste [Avo 1-5, Bür 1-5, Cro 5, Has 1-2, Hof 2-5, Li 1-4, Mor 1-2, Nag, Ros, Sch 1-3, Sei]

[SW 2] Referenzenliste [Avo 3-4, Bes 1, Bür 1-2, Che 1-2, Fuj 1-3, Has 2, Li 3-4, Nej]

[SW 3] Referenzenliste [Bür 5, Che 1, Has 1-2, Hör 1-2, Höv

[SW 4] Referenzenliste [Bri 1-3, Fui 4-5, Han, Hof 1, Pet 1-4] 
[Swa] L. W. Swanson und L. C. Crouser, Anomalous Total Energy Distribution for a Tungsten Field Emitter, Physical Review Letters 16, 389 (1966); Physical Review 163, 622 (1967)

[Tak] Noboru Takeuchi, C. T. Chan und K. M. Ho, Au(111): A theoretical study of the surface reconstruction and the surface electronic structure, Physical Review B 43, 13899 (1991)

[Tal] W. H. F. Talbot, Facts relating to optical science, No. IV, Philosophical Magazine 9, 401 (1836)

[Tam 1] I. Tamm, Über eine mögliche Art der Elektronenbindung an Kristalloberflächen, Zeitschrift für Physik 76,849 (1932)

[Tam 2] I. Tamm, Über eine mögliche Art der Elektronenbindung an Kristalloberflächen, Physikalische Zeitschrift der Sowjetunion 1, 733 (1932)

[Tec] Tectra GmbH, Reuterweg 65, D-60323 Frankfurt, Tel. 069-720040, www.tectra.de

[Ter 1] J. Tersoff und D. R. Hamann, Theory and Application for the Scanning Tunneling Microscope, Physical Review Letters 50, 1998 (1983)

[Ter 2] J. Tersoff und D. R. Hamann, Theory of the scanning tunneling microscope, Physical Review B 31, 805 (1985)

[Ter 3] J. Tersoff, Theory of the scanning tunneling microscope, Structure of Surfaces xii, S. 54, SpringerVerlag (Berlin 1985)

[The] N. Theuerkauf, Spaltstufen auf GaAs, Diplomarbeit, IV. Physikalisches Institut der Universität Göttingen (Gruppe R. G. Ulbrich, 1997)

[Wah] E. Wahlström, I. Ekvall, H. Olin und L. Walldén, Long-range interaction between adatoms at the Cu(111) surface imaged by scanning tunnelling microscopy, Applied Physics A 66, S1107 (1998)

[Wat] G. N. Watson, A treatise on the theory of Bessel functions, $2^{\text {nd }}$ ed., Cambridge university press (Cambridge 1944)

[Wea] J. M. R. Weaver, L. M. Walpita und H. K. Wickramasinghe, Optical absorption microscopy and spectroscopy with nanometer resolution, Nature 342, 783 (1989)

[Wie] R. Wiesendanger, Scanning probe microscopy and spectroscopy : methods and applications, Cambridge University Press (Cambridge 1994)

[Wil 1] C. C. Williams und H. K. Wickramasinghe, Microscopy of chemical-potential variations on an atomic scale, Nature 344, 317 (1990)

[Wil 2] C. C. Williams und H. K. Wickramasinghe, Scanning chemical potential microscope: A new technique for atomic scale surface investigation, Journal of Vacuum Science and Technology B 9, 537 (1991)

[Wil 3] C. C. Williams und H. K. Wickramasinghe, Scanning Thermal Profiler, Applied Physics Letters 49, 1587 (1986); Microelectronic Engineering 5, 509 (1986)

[Wöl] Ch. Wöll, S. Chiang, R. J. Wilson und P. H. Lippel, Determination of atom positions at stacking-fault dislocation on Au(111) by STM, Physical Review B 39, 7988 (1989)

[Woo] D. P. Woodruff, W. A. Royer und N. V. Smith, Empty surface states, image states, and band edge on $A u(111)$, Physical Review B 34, 764 (1986)

[Xu 1] J. Xu, B. Koslowski, R. Möller, K. Läuger, K. Dransfeld und I. H. Wilson, Proposal to study the thermopower produced by a vacuum-tunneling junction, Journal of Vacuum Science and Technology B 12, 2156 (1994)

[Xu 2] J. B. Xu, K. Läuger, R. Möller, K. Dransfeld und I. H. Wilson, Heat transfer between two metallic surfaces at small distances, Journal of Applied Physics 76, 7209 (1994)

[Xu 3] J. B. Xu, Heat transfer between two metallic surfaces at small distances, Dissertation, Universität Kon$\operatorname{stanz}(1993)$

[Xu 4] J. B. Xu, K. Läuger, R. Möller, K. Dransfeld und I. H. Wilson, Energy-exchange processes by tunneling electrons, Applied Physics A 59, 155 (1994)

[Yel] K. Baier. OFR. u. Akademischer Ritter von Yelin, Der Thermo-Magnetismus der Metalle, Annalen der Physik 73, 415 (1823)

[Zan] Andrew Zangwill, Physics at surfaces, Cambridge University Press (Reprint, Cambridge 1996)

[Zim] J. M. Ziman, Principles of the Theory of Solids, Cambridge University Press (Cambridge 1979); deutsche Übers.: Prinzipien der Festkörpertheorie, hrsg. P. Rennert, Deutsch-Verlag (Thun 1999) 


\section{Danksagung}

An dieser Stelle möchte ich mich herzlich bei allen bedanken, die mit Rat und Tat zum Gelingen dieser Arbeit beigetragen haben.

Herrn Prof. Dr. R. G. Ulbrich danke ich für die Ermöglichung dieser Dissertation sowie für seine stete Diskussionsbereitschaft. Die breite physikalische Ausbildung, die ich in den Jahren meiner Tätigkeit im IV. Physikalischen Institut erhalten durfte, und die Förderung einer gesunden kritischen Betrachtungsweise wird mir auch in Zukunft sehr nützlich sein.

Herrn Prof. Dr. K. Schönhammer danke ich für die Übernahme des Korreferats und das Interesse an meiner Arbeit. Den Herren Prof. Dr. W. Felsch, Prof. Dr. H. Hofsäß, Prof. Dr. R. Kirchheim und Prof. Dr. Schmahl danke ich für die Mitarbeit in der Prüfungskommission.

Mein größter Dank gilt Herrn Dr. Martin Wenderoth, der mich in den letzten Jahren immer wieder auf den richtigen Weg zurückbrachte, wenn ich mich mal wieder im Sumpf der Details verirrt hatte. Nicht nur die richtige Mischung aus Humor und gepflegter Streitkultur, sondern auch sein enormes Fachwissen und unermüdlicher Laboreinsatz ist für diese Arbeit eine unschätzbare Stütze gewesen. Vielen Dank, Martin!

Dank gilt auch meinen tapferen Mitstreitern im Labor für eine stets freundschaftliche Zusammenarbeit und immerwährende Hilfsbereitschaft. Namentlich zu nennen sind hier (in alphabetischer Reihenfolge) Jens Garleff für zahlreiche philosophische Dispute in der Mittagspause, Sebastian Kagel für das Informieren über neueste technische Errungenschaften, meinen Mitdoktoranden Norbert Quaas, Thilo Reusch und Katharina Sauthoff für die Kameradschaft, die fleißige Mitarbeit im Labor sowie die Übernahme vieler zeitraubender Nebentätigkeiten in der Endphase meiner Arbeit.

Auch meinen Laborgenossen aus alten Tagen, Dr. Andreas Heinrich, Dr. Matthias Rosentreter, Dr. Alexander Schneider und Dr. Ralf Ziebold möchte ich ein Dankeschön für viele gesellige Stunden aussprechen. Ein besonderes Dankeschön geht hier an Alexander, der mit vielen hilfreichen Tipps \& Tricks einen Beitrag zum Gelingen dieser Arbeit geleistet hat.

Ebenfalls ein herzliches Dankeschön geht an Bernhard Spicher für ausgefeilte Ingenieurskunst, die Unmögliches häufig doch möglich gemacht hat und auch ansonsten das Leben im Labor mit einem unerschöpflichen Repertoire an Erfahrung (Kabelbinder...) angenehm bereicherte.

Bei Frau Gerda Brocks möchte ich mich für ihr Engagement im SFB, ihre Herzlichkeit und ihren Einsatz ebenfalls herzlich bedanken. 
Herrn Klaus Langohr möchte ich stellvertretend für alle Mitarbeiter in der Elektronikwerkstatt für die zahlreichen elektronischen Basteleien und Entwicklungen danken, ohne die ein Gelingen dieser Arbeit natürlich nur schwerlich möglich gewesen wäre.

Derselbe Dank geht auch an Herrn Uwe Frenzel stellvertretend für die gesamte feinmechanische Werkstatt für die immer prompte Bearbeitung vieler wichtiger „Kleinigkeiten“.

Herrn Dr. Hans D. Schulte, Frau Edith Rohrmoser, Frau Miriam Müller-Reimann und ausnahmslos allen anderen hier nicht genannten Mitarbeitern des IV. Physikalischen Institutes bedanke ich mich für die reibungslose Zusammenarbeit.

Bei all meinen Freunden außerhalb des Institutes möchte ich mich für ihre langjährige Treue, die auch während meiner arbeitsintensiven Zeit an meiner Dissertation standhielt, herzlich bedanken.

Bei meiner Freundin Silvana Arsova möchte ich mich für ihr Verständnis, ihre Geduld, vor allem aber ihre seelische Unterstützung und ihren festen Glauben an mich während der vielen Jahre auf das Innigste bedanken. Ohne Dich, Silvana, hätte ich die Zeit der Dissertation nicht mit dieser Kraft und inneren Stärke durchgestanden.

Auch meinen lieben Eltern, Gerd und Adelheid Engel, drücke ich hiermit meine tiefste Dankbarkeit für die immerwährende Unterstützung und Hilfe in allen Lebenslagen aus. 


\title{
Lebenslauf
}

\author{
Klaus Jürgen Engel \\ Geboren am 07.08.1969 in Mönchengladbach/Rheydt \\ Staatsangehörigkeit: deutsch
}

Aug. 1976 - Juni $1980 \quad$ Grundschule in Mönchengladbach/Rheydt

Aug. 1980 - Okt. $1980 \quad$ Hugo-Junkers-Gymnasium in Mönchengladbach/Rheydt

Okt. 1980 - Juni $1989 \quad$ Wilhelmsgymnasium in Kassel mit Abiturabschluss

Juli 1989 - Sept. $1990 \quad$ Zivildienst

Okt. 1990 - Okt. $1992 \quad$ Grundstudium Physik an der Universität Göttingen mit Vordiplom-Abschluss

Okt. 1992 - Okt. 1996 Hauptstudium Physik an der Universität Göttingen

Okt. 1996

Diplomarbeit am IV. Physikalischen Institut der Universität

Göttingen, Titel: „Thermoelektrische Kontrasteffekte in der Rastertunnelpotentiometrie““

Seit Jan. 1997

Wissenschaftlicher Mitarbeiter beim IV. Physikalischen Institut der Universität Göttingen

Sept. 2001

Fertigstellung der Doktorarbeit: „Raster-ThermospannungsMikroskopie der Interferenz von Elektronenwellen auf der Au(111)-Oberfläche“

Göttingen, 13. 09. 2001 